\title{
The Arabidopsis C/S1 bacic leucine Zipper transcription factor network: \\ Impact of heterodimer formation on target gene transcription
}

\author{
Dissertation \\ zur Erlangung des Doktorgrades \\ der Mathematisch-Naturwissenschaftlichen Fakultäten \\ der Georg-August-Universität zu Göttingen
}

vorgelegt von

Andrea Ehlert

aus Bad Saarow-Pieskow

Göttingen 2009 
Referent:

Korreferent:

Tag der mündlichen Prüfung:
PD. Dr. Wolfgang Dröge Laser

Prof. Dr. Christiane Gatz

20.01.2010 
Träume nicht Dein Leben, sondern lebe Deinen Traum! 


\section{Table of Content}

1 Summary …................................................................................................................. 1

2 General Introduction............................................................................................... 3

2.1 Basic leucine zipper (bZIP) transcription factors ............................................ 3

2.2 The activity of bZIP transcription factors is frequently modulated by posttranslational mechanisms and heterodimerisation...................................... 5

2.3 The C/S network of Arabidopsis bZIP transcription factors .............................. 6

2.4 Function of group $C$ and $S$ bZIPs in primary metabolism and low energy

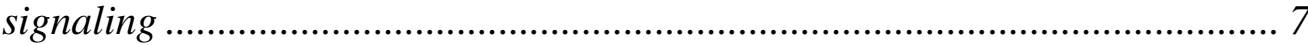

2.5 Impact of group C bZIPs as transcriptional regulators of seed maturation

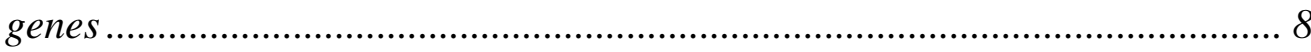

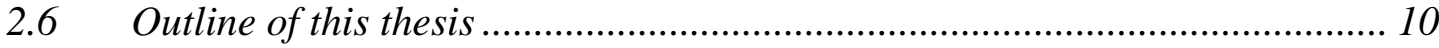

3 Members of the bZIP C/S1 network form specific heterodimers ...................... 13

4 Members of the C/S1 bZIP network show overlapping expression pattern ... 24

5 bZIP heterodimers induce transcriptional activity of target genes in hypoosmotic response................................................................................................... 37

6 Heterodimers of Arabidopsis transcription factors bZIP1 and bZIP53 are reprogramming amino acid metabolism during low energy stress

7 Heterodimers of the C/S1 bZIP network controls gene expression in seed maturation

8 Genome-wide analysis reveals the impact of bZIP heterodimers on target promoter selection

$9 \quad$ General discussion .................................................................................................... 93

9.1 Specific heterodimerisation of group $C$ and group S1 bZIP transcription factors is mediated by the leucine zipper.

9.2 Expression patterns control abundance of bZIP monomer and therefore heterodimer formation.

9.3 Heterodimerisation between members of the C/S1 network controls target gene expression in stress response, primary metabolism and seed development 
9.4 C/S1 heterodimer formation controls DNA binding and interactions with other transcription factors

9.5 Members of the C/S1 heterodimers differ in their properties but show partial redundancy

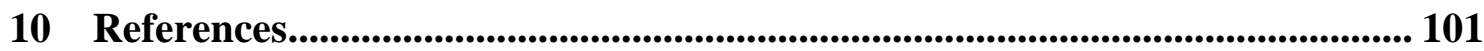

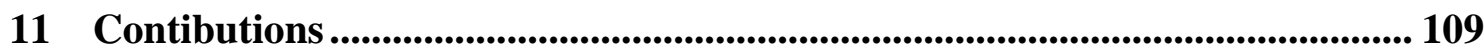

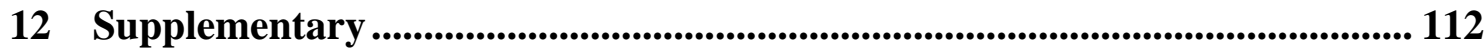

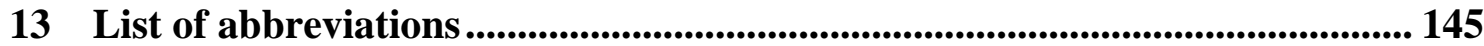

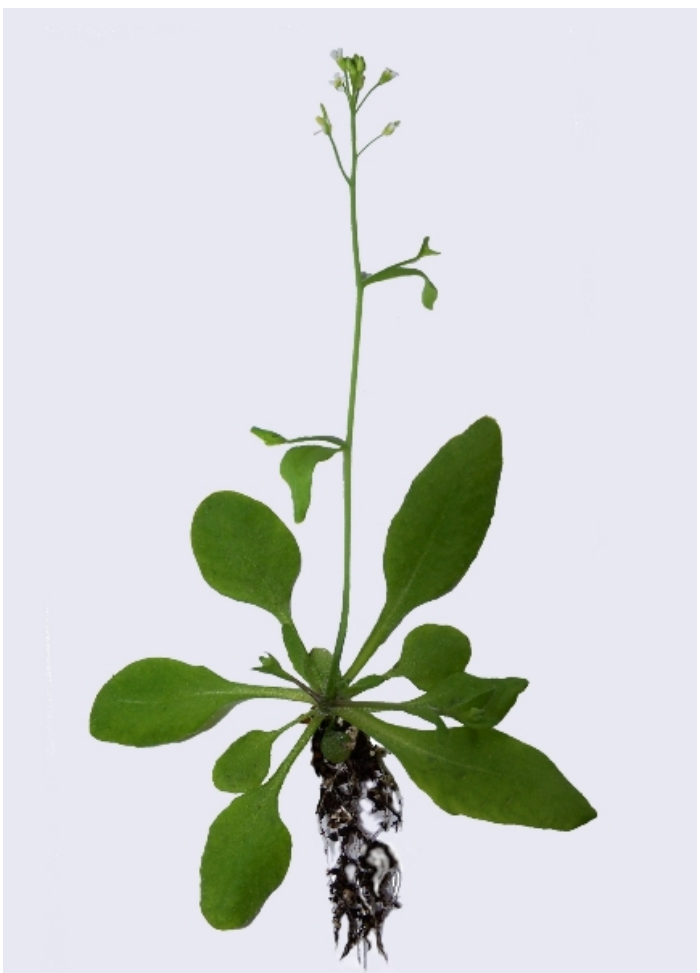

Arabidopsis thaliana (Ackerschmalwand) 


\section{Summary}

Heterodimerisation of basic leucine zipper (bZIP) transcription factors (TFs) is proposed to play a crucial role in regulation of gene expression. To analyse bZIP heterodimerisation of Arabidopsis thaliana (At) bZIPs, a Gateway ${ }^{\circledR}$-based two-hybrid system in plant protoplasts has been established and obtained data has been compared to results of the classical $\mathrm{Y} 2 \mathrm{H}$ approach (EHLERT et al., 2006).

Specific high-affinity heterodimerisation could be observed between nine bZIP members of the Arabidopsis thaliana group $\mathrm{C}$ and S1 bZIP TF indicating a functional connection and it is therefore, these bZIPs are called the C/S1 bZIP network. Heterodimerisation should be limited by abundance of the protein partners. Accordingly, using bZIPpromoter:GUS lines, the different bZIPs shows partly overlapping and distinct expression patterns (WELTMEIER et al., 2009).

The functional impact of bZIP heterodimerisation has been demonstrated for the ProDH gene encoding Proline Dehydrogenase a central enzyme in proline degradation during rehydration after recovery from osmotic stress. ProDH is a direct target gene of the group S1 bZIP transcription factor AtbZIP53. Dimerisation studies show a synergistic enhancement of target gene activation with the group $\mathrm{C}$ member AtbZIP10. This heterodimer induced transactivation is independent of the DNA binding activity mediated by the basic domain and appears to be a crucial mechanism to modulate transcription factor activity (WELTMEIER et al., 2006).

Furthermore, low energy stress administered by extended darkness leads to activation of AtbZIP1 and AtbZIP53, which by heterodimerisation regulate several genes in amino acid metabolism (DIETRICH et al., unpublished).

In addition, an influence on the transcription of maturation (MAT) genes could be shown for AtbZIP53. Heterodimerisation was demonstrated to enhance bZIP protein stability, DNA binding to a G-box element and activation of MAT promoters. Thus target gene activation strongly correlates with the ratio of the correspondent bZIP heterodimerisation partners AtbZIP10 and AtbZIP25. Interestingly AtbZIP53 is not able to directly interact with ABI3, a crucial transcriptional regulator in Arabidopsis seeds. Furthermore we had the possibility to show that the AtbZIP53/10 heterodimer can form a ternary complex with 


\begin{abstract}
$\mathrm{ABI} 3$ and activate the expression of MAT genes in plants in comparison to the AtbZIP53/10 heterodimer itself (ALONSO et al., 2009).

To decipher the complex network of different bZIP heterodimers, which can bind and regulate particular sub-sets of target genes, Arabidopsis mesophyll protoplasts were transformed with plasmid DNA encoding the group C transcription factor AtbZIP10, the group S1 AtbZIP11, or both. Global gene expression analysis revealed that co-expression of AtbZIP11 and AtbZIP10 results in substantial differences in up-regulated gene sets if compared to single bZIP expression. Altogether these data provide conclusive evidence that bZIP heterodimerisation acts as an efficient mechanism to control target gene expression in plants (HANSSEN et al., unpublished).
\end{abstract}




\section{General Introduction}

Due to their settled life style plants are unable to avoid unfavorable environmental conditions, such as infection with pathogens or abiotic stresses such as low temperature or high salinity. In response to changing environmental conditions, plants continuously have to adjust their metabolism to survive (CHASAR, 2002). Hence, perception, integration and processing of environmental information are essential requirements for plant growth and development. In part, these responses are mediated on gene expression level controlled by transcription factors (TFs). These proteins contain a DNA-binding domain, which bind specific DNA-cis-elements of target gene promoters. Furthermore, the TFs possess regulatory domains (PTASHNE and GANN, 1997). Due to interaction with proteins of the transcriptional machinery, TFs can either function as activators of gene expression or as repressors. The repression can be active or passive (THIEL et al., 2004).

TFs, which are classified by their DNA binding domains, generally form large families such as homeobox, helix- loop- helix, zinc finger or basic leucine Zipper (bZIP) TFs (RIECHMANN et al., 2000; LANDSCHULZ et al., 1988).

\subsection{Basic leucine zipper (bZIP) transcription factors}

BZIP proteins are ubiquitously found in all eukaryotic species (LANDSCHULZ et al., 1988). In general, bZIP TFs harbour a basic domain (b), followed by a zipper dimerisation domain (ZIP), which form an $\alpha$ helical structure (HURST, 1995). The leucine zipper consists of heptad repeats of leucine or other bulky hydrophobic amino acids (aa) such as phenylalanine, methionine, valine and isoleucine (BAXEVANIS and VINSON, 1993; LANDSCHULZ et al., 1988). The seven aa of one heptad repeat are labeled according to the nomenclature of McLACHLAN and STEWARD (1975) as a, b, c, d, e, f, and g (Figure 1). At position $d$ is the aa leucine or other hydrophobic aa located. These hydrophobic aa are arranged at one side of the helix forming a hydrophobic domain which enables intermolecular homo- or heterodimerisation (LANDSCHULZ et al., 1988; O'SHEA et al., 1989) (Figure 1). The dimerisation of two $\alpha$-helixes results in the generation of a coiledcoil formation (BAXEVANIS and VINSON, 1993; LUPAS, 1996). The dimerisation is 
obviated, if the aa on position e repels the aa at position $\mathrm{g}$. Hence, dimerisation is very specific (SIBERIL et al., 2001; VINSON et al., 2002). Formation of homo- or heterodimers provides huge combinatorial flexibility to a regulatory system (SIBERIL et al., 2001; VINSON et al., 2002).

(d)

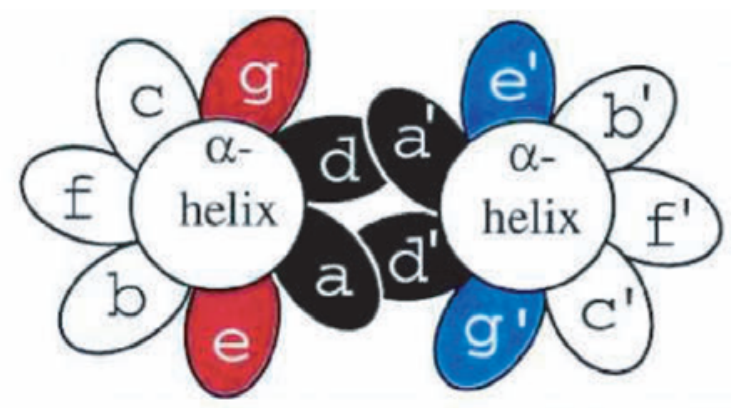

Figure 1 EHLERT et al., 2006; Figure 1d

Demonstration of the aa positions in bZIP dimers.

Heterodimerisation of two bZIP proteins is mediated by interaction/repulsion of the aa located in positions a and $\mathrm{d}$ as well as e and g (DEPPMANN et al., 2004; FONG et al., 2004).

The zipper-domain is fused to a basic domain (b) which mediates binding to specific promoter cis-elements containing ACGT-related motifs (SCHINDLER et al., 1992; DE PATER et al., 1994; SATHO et al., 2004). Furthermore, sequences flanking the ACGT-core motif are also important for efficient DNA binding (FOSTER et al., 1994). Additionally, as demonstrated for mammalian, yeast, amphibian and plant nuclear proteins, the basic domain can harbour a nuclear localisation sequence (NLS) (VAN DER KROL and CHUA, 1991; VARAGONA and RAIKHEL, 1994; LARA et al., 2003).

Members of the bZIP TF family play crucial roles in almost all biological processes.

For example, in growth factor- stimulated mammalian cells the c-JUN binds DNA as a dimer with c-FOS and regulates transcription (HALAZONETIS et al., 1988; ABATE et al., 1991). In yeast (Saccharomyces cerevisiae) the bZIP TFs GCN4 (General Control Nondepressible 4) controls transcriptional regulation of aa metabolism and whereas YAP-1 is involved in abiotic stress response, respectively (VOGT et al., 1987; MOYE-ROWLEY et al., 1989).

The functional role of bZIP TFs in plants has been linked to a large diversity of biological aspects, for example responses to light (OYAMA et al., 1997; SCHULZE- LEFERT et al., 1989a), hormones (FUKAZAWA et al., 2000), biotic (ZHOU et al., 2000) and abiotic stresses (AGUAN et al., 1993), as well as cell proliferation (MIKAMI et al., 1995) and developmental processes (CHUANG et al., 1999). 


\subsection{The activity of bZIP transcription factors is frequently modulated by posttranslational mechanisms and heterodimerisation}

In plants, various posttranslational mechanisms have been shown to alter the activity of bZIP TFs as it has been recently reviewed by SCHÜTZE et al 2008. TFs are often phosphorylated, which might regulate nuclear transport, DNA binding properties or protein stability (CICERI et al., 1997). Protein phosphorylation and dephosphorylation, catalysed by protein kinases and phosphatases, respectively, is an important control mechanism for several biological processes in yeast, plant, and animal cells (CICERI et al., 1997). The maize bZIP TF Opaque $2(\mathrm{O} 2)$ has been shown to be involved in controlling seed specific expression (VICENTE- CARBAJOsA et al., 1997). Depending on the day- night cycle O2 exhibits in vivo a different phosphorylation pattern which modulate its DNA binding affinity (CICERI et al., 1997). In Arabidopsis thaliana some bZIP TFs of the group A require $\mathrm{ABA}$ (abscisic acid) dependent phosphorylation to be transcriptional active (CHAE et al., 2007). Furthermore, nuclear localisation of AtbZIP51 (VIP1) is dependent on phosphorylation (DJAMEl et al., 2007). The phosphorylation of AtbZIP56 (HY5) and AtbZIP39 (ABI5) influences protein stability (JAKOBY et al., 2002).

BZIP factors might also be regulated by intracellular partitioning (SCHÜTZE et al., 2008). For example AtbZIP10 is regulated in its subcellular localisation through shuttling between cytoplasm and nucleus. The control of this shuttling is mediated by AtbZIP10 binding protein LSD1 (Lesions Simulating Disease Resistance 1) (KAMINAKA et al., 2006).

Heterodimerisation is a typical mechanism for regulating bZIP factor activity as it has been described for mammalian TFs such as JUN and FOS (HALAZONETIS et al., 1988). As a homodimer, c-JUN binds to the AP-1 cis-element, while c-FOS fails to dimerise and displays no apparent affinity for the AP-1 element. However, $c-J U N$ and $c-F O S$ protooncogenes form a heterodimeric complex and regulate transcription via the AP-1 binding site. In plants, heterodimerisation between the maize bZIP factors O2 and OHP1/2 (PYSH et al., 1993) or the orthologous proteins from barley has been described to function in the regulation of seed maturation (MAT) genes (VICENTE-CARBAJOSA et al., 1998). The rice bZIP TF LIP19 is induced by low-temperature and heterodimerises with OsOBF1 (Oryza sativa OBF1) in response to cold treatment (SHIMIzI et al., 2005). Furthermore, 
specific bZIP heterodimerisation has been demonstrated between tobacco (Nicotiana tabacum) BZI-1 and a group of low molecular weight bZIPs, namely BZI-2, BZI-3 and BZI-4 (STRATHMANN et al., 2001). The same heterodimerisation specificity has been described for the highly homologous CPRF (Common Plant Regulatory Factor) TFs isolated from parsley (Petroselium crispum) (ARMSTRONG et al., 1992; RÜGNER et al., 2001). Therefore, it is resonably to speculate that heterodimerisation plays a crucial role in the regulation of transcriptional activation by these bZIPs.

\subsection{The C/ S network of Arabidopsis bZIP transcription factors}

In the model plant Arabidopsis thaliana, the bZIP TF family comprises 75 members which have been classified into 10 groups (JAKOBY et al., 2002). Tobacco BZI-1 shares highest homology with the four group C bZIP TFs (AtbZIP9, AtbZIP10, AtbZIP25 AtbZIP63) (STRATHMANN et al., 2001). Members of this group show a molecular weight of 30-40 $\mathrm{kDa}$ and are characterised by an extended zipper domain with nine heptad repeats. The group C TFs exhibit a conserved intron-exon-structure (HEINEKAMP et al., 2002). AtbZIP10 and AtbZIP25 are the closest homologues to O2 from maize (VICENTECARBAJOSA et al., 1998; ONATE et al., 1999; ONODERA et al., 2001; LARA et al., 2003). BZI-2, BZI-3 and BZI-4 related Arabiodpsis proteins belong to the large group S of bZIP TFs which contains 17 small TFs with a molecular weight of $15-20 \mathrm{kDa}$. These TFs contain a short N- and C-terminal region consisting of a central basic domain and an extraordinary long leucin zipper with eight to nine heptad repeats (JАковY et al., 2002). The group $\mathrm{S}$ bZIP TFs possess no introns. Based on their sequence homology the group S bZIP- TF can be divided into three subgroups (EHLERT et al., 2006). Remarkably, all members of subgroup S1 (AtbZIP1, AtbZIP2, AtbZIP11, AtbZIP44, AtbZIP53) contain highly conserved upstream open reading frames (uORFs) in the 5 region of their mRNAs mediating a "sucrose induced repression of translation" (SIRT) (ROOK et al., 1998; WIESE et al,. 2004; WELTMEIER et al., 2009). Although not firmly established, AtbZIP11 (ATB2) has been proposed to be involved in source-sink control (ROOK et al., 1998). Furthermore, group S bZIPs and related TFs from other plant species are implicated in various stress responses (AGUAN et al., 1993; KUSANO et al., 1995; YANG et al., 2003; LEE et al., 2002). Based on preliminary data (EHLERT et al., 2006), heterodimerisation of the Arabidopsis 
group $\mathrm{C}$ and $\mathrm{S}$ members seems to occur as it has been described for the tobacco and parsley system. However, a detailed analysis is necessary to define the heterodimerisation properties of these bZIPs.

\subsection{Function of group $C$ and $S$ bZIPs in primary metabolism and low energy signaling}

Plants sustain life on Earth by converting solar energy. Despite its fundamental importance, little is known about adaptation mechanisms of plants to the daily light/dark cycle, or how they respond to unpredictable environmental stresses that compromise photosynthesis and deplete energy supplies (for review see BAENA- GONZALEZ et al., 2007). Fluctuation of energy status is an inherent part of plant lifestyle and can be caused by alterations of the normal day-night cycle due to shading or an extension of the night hours (SMITH and STITT 2007). An energy deficit can also be triggered by carbon hijack by pathogens or many adverse conditions such as drought, temperatures extremes, pollutants or flooding that interfere with carbon assimilation and/or respiration (BASSHAM et al., 2006). The response to energy fluctuation is triggered before a complete energy deficit occurs. It is particularly important to consider that even short periods of severe carbon starvation lead to an inhibition of growth (GUILIONI et al., 2003; SMITH and STITT, 2007). Comparative studies of public microarray data have uncovered that this is in part accomplished through changes in gene expression (CROSS et al., 2006; BAENAGONZALEZ and SHEEN, 2008). The emerging view is that this transcriptome reprogramming in energy and stress signaling is partly regulated by the evolutionary conserved energy sensor protein kinases, SNF1 (Sucrose Non- Fermenting 1) in yeast, AMPK (AMP- activated protein kinase) in mammals and SnRK1 (SNF1- related protein kinase 1) in plants (for review see POLGE and THOMAS, 2007). Upon sensing the energy starvation associated with stress, nutrient deprivation and darkness, SnRK1 triggers extensive transcriptional changes that contribute to restoring homeostasis, promoting cell survival and elaborating longer-term responses for adaptation, growth and development (HALFORD et al., 2003; HRABAK et al., 2003; POLGE and THOMAS 2007; HALFORD et al., 2004; HARDIE, 2007). Recently, the Arabidopsis thaliana SnRK1-like kinases AKIN10 and AKIN11 have been proposed to function as central signaling integrators mediating 
adaptation to energy deprivation (BAENA-GONZALEZ et al., 2007). In Arabidopsis thaliana, a target of AKIN10/ AKIN11 is the asparagine synthetase (ASN1), which encodes the final enzymatic step in asparagine biosynthesis to control the level of asparagine (Asn) (BAENA-GONZALEZ et al., 2007). In comparison to glutamine, asparagine contains more nitrogen than carbon and is therefore used to store and transport nitrogen, especially under stress conditions such as prolonged darkness, when carbon is limiting (LAM et al., 1994). Sequence analysis of the ASN1 promoter revealed two G-boxes (CACGTG). This cis-element is known to be bound by bZIP TFs (NIU et al., 1999). Protoplast based functional screens revealed that the activation of promoter luciferase constructs $\left(\right.$ Pro $\left._{\mathrm{ASN} 1}: \mathrm{LUC}\right)$ by AKIN10 and AKIN11 is mediated by one of these G-boxes (BAENA-GONZALEZ et al., 2007). Furthermore, several group S1 members have been shown to mediate ASN1 activation in protoplasts (BAENA-GONZALEZ et al., 2007). However, functional characterisation of bZIP factors in low energy stress response making use of whole plant systems and gain-of-function and loss-of-function approaches have not been performed yet.

\subsection{Impact of group C bZIPs as transcriptional regulators of seed maturation genes}

Storage reserves accumulate in form of carbohydrates and proteins during seed development (LARA et al., 2003). Degradation of these reserves upon germination provides nutrients to the growing seedling before the photosynthetic capacity is fully acquired (LARA et al., 2003). Expression of seed maturation (MAT) genes in the developing seed is tightly regulated and coordinated with primary metabolism, in particular aa metabolism (HUGHES et al., 1989; GUTIERREZ et al., 2007). The MAT genes include major seed storage protein (SSP) and late embryogenesis abundant (LEA) genes. SSP genes (like 2S2 albumin) are expressed at early and mid-maturation phases and encode proteins used as nutrient resources, whereas the $L E A$ genes are primarily proposed to function in acquisition of desiccation tolerance, which are expressed at later stages of maturation (wOBUS et al., 1999; HOEKSTRA et al., 2001). Expression of SSP genes is controlled on a transcriptional level and has been intensively studied as a model system for developmentally and tissuespecific gene regulation (VICENTE-CARBAJOSA and CARBONERO, 2005). The 
Arabidopsis 2S2 albumin gene promoter contains the RY (CATGCATG) motif (LARA et al., 2003). Remarkably, factors with a B3 domain such as ABI3, FUS3, or LEC2, are implicated in the regulation of SSP genes via the RY element (LARA et al., 2003; VICENTECARBAJOSA and CARBONERO, 2005). Additionally, a G-box related cis-element (CACGTG) is conserved in many SSP genes, which is the typical binding site of bZIP TFs. Actually, the first functionally characterised bZIP TF in plants was $\mathrm{O} 2$ from maize, a potent regulator of SSP genes (LARA et al., 2003). The Arabidopsis genome contains four genes encoding O2 related bZIPs, namely AtbZIP9, AtbZIP10, AtbZIP25 and AtbZIP63 (LARA et al., 2003). However, only the highly homologous TFs AtbZIP10 and AtbZIP25 are expressed in the developing seed and bind the G-box in the SSP promoter of 2S2 albumin and Cruciferin3 (LARA et al., 2003). Therefore, these bZIPs are proposed to fulfill functions, assigned to the monocot TF O2 (LARA et al., 2003). Yeast-two-hybrid (Y2H) analysis revealed that AtbZIP10 and AtbZIP25 can interact with ABI3, an important regulator of gene expression in the seed of Arabidopsis (LARA et al., 2003). When expressed individually, AtbZIP10, AtbZIP25 and ABI3 could not significantly activate SSP promoters. However, co-expression of AtbZIP10 or AtbZIP25 with ABI3 resulted in a remarkable increase in the activation of SSP promoters, suggesting that they are part of a regulatory complex involved in seed-specific expression (LARA et al., 2003). Although an important function in MAT gene regulation can be assigned to AtbZIP10 and AtbZIP25, it has to be analysed whether other bZIP heterodimerisation partners might be involved in this process. 


\subsection{Outline of this thesis}

Based on previous work in other plant species, Arabidopsis group $\mathrm{C}$ and $\mathrm{S}$ bZIPs might form heterodimers which would consequently influence their functional impact on gene regulation. Therefore, this thesis focuses on (1) evaluation of bZIP heterodimerisation capacity of all group C and S members, (2) expression studies for the corresponding genes to evaluate co-localisation of bZIP heterodimerisation partners, (3) the functional impact of bZIP heterodimers on target gene regulation and (4) a protoplast-based transfection approach to evaluate the impact of bZIP heterodimers on a genome-wide level. These topics are presented in the following publications and manuscripts.

\section{Chapter 3: (EHLERT et al., 2006)}

\section{Members of the bZIP C/S1 network form specific heterodimers}

In order to study in vivo heterodimerisation of bZIP TFs, an Arabidopsis protoplasts twohybrid system $(\mathrm{P} 2 \mathrm{H})$ has been established based on yeast Gal4 DNA binding (BD) and activation domains (AD). To test the efficiency of the system, a homo- and heterodimerisation matrix of Arabidopsis group C and S bZIP TFs has been established and compared to results obtained in yeast two- hybrid systems (Y2H). A preference in heterodimerisation between members of the sub group S1 and group C was observed.

\section{Chapter 4: (WELTMEIER et al., 2009)}

\section{Members of the C/S1 bZIP network show overlapping expression pattern}

The group $\mathrm{C} / \mathrm{S} 1 \mathrm{TF}$ network is proposed to implement transcriptional reprogramming in response to environmental stresses. In order to form heterodimers, the group C and S1 bZIP TFs have to be co-expressed and co-localised. Hence, co-expression was studied making use of AtbZIPpromoter:GUS lines and array expression data. Clearly overlapping and distinct differences in expression patterns were observed for the nine members of the $\mathrm{C} / \mathrm{S} 1$ heterodimerisation network. 
Chapter 5: (WELTMEIER et al., 2006)

bZIP heterodimers induce transcriptional activity of target genes in hypoosmotic response

In response to osmotic stress, many plants accumulate the compatible and toxic osmolyte proline (Pro) (DELAUNEY et al., 1993; LIU and ZHU, 1997; PARVANOVA et al., 2004). Degradation of Pro is catalysed by the enzymes proline dehydrogenation (ProDH) and P5C (Pyrrolin-5-carboxylate) dehydrogenase (5PCDH) (PENG et al., 1996; VERBRÜGGEN et al., 1996; NAKASHIMA et al., 1998; YOSHIBA et al., 1999). The transcriptional activation of the ProDH gene by hypo-osmolarity is dependent of an ACTCAT cis-element, which is a typical binding site of bZIP TFs. Dimerisation studies using yeast and Arabidopsis protoplast-based two-hybrid $(\mathrm{P} 2 \mathrm{H})$ systems and $\mathrm{BiFC}$ (bimolecular fluorescence completition) reveal that AtbZIP53 preferentially forms heterodimers with the members of group C bZIPs, in particular with AtbZIP10. This heterodimer mediated a significant activation of ProDH transcription independent of the basic DNA binding properties. Heterodimerisation of bZIP TF has been demonstrated to function as a crucial mechanism to modulate TF activity and function.

\section{Chapter 6: (DIETRICH et al., unpublished)}

\section{Heterodimers of the C/S1 bZIP network controls gene expression in low energy stress response}

In plants, energy deprivation leads to dramatic reprogramming of transcription. Growth under extended night conditions results in energy starvation and activation of the group S1 bZIP TFs AtbZIP1 and AtbZIP53 by transcriptional and posttranscriptional mechanisms. Both TFs control the expression of a particular subset of genes involved in the biosynthesis of the aa asparagine, proline, valine, leucine and isoleucine. Hence, group S1 members are implicated in reprogramming of primary metabolism in response to low energy stress. Direct binding of the bZIP TFs to promoters of selected aa metabolic genes has been confirmed by Chromatin Immuno Precipitation (ChIP) and promoter analysis defining Gand ACTCAT-boxes as crucial cis-elements in low energy stress response. 
Chapter 7: (ALONSO et al., 2009)

Heterodimers of the C/S1 bZIP network controls gene expression in seed maturation

Arabidopsis seed maturation (MAT) genes are transcriptionally controlled by members of several TF families, like bZIPs, B3s, MYBs and DOFs. Group S1 AtbZIP53 was defined as a previously undescribed regulator of MAT genes. Heterodimers of AtbZIP53 and group C AtbZIP10 and AtbZIP25 were identified to synergistically activate MAT gene expression. Heterodimerisation was demonstrated to enhance bZIP protein stability, DNA binding to a G- box element and activation of a SSP promoter (2S2 albumin). Furthermore, AtbZIP53 alone does not interact with ABI3, another crucial transcriptional regulator in Arabidopsis seeds, but ternary complex formation between the AtbZIP53/10 heterodimer and ABI3 leads to increased 2S2 albumin gene expression.

\section{Chapter 8: (HANSSEN et al., unpublished)}

Genome-wide analysis reveals the impact of bZIP heterodimers on target promoter selection

AtbZIP proteins include a large family of TFs, which affect gene expression by DNA binding as homo- or heterodimer. To decipher the complex network of different AtbZIP dimers which can bind and regulate particular sub-sets of target genes, Arabidopsis mesophyll protoplasts were transformed with plasmid DNA encoding the group C TF AtbZIP10 and the group S1 AtbZIP11, both and non heterodimerising derivatives. The array analysis revealed that co-expression of AtbZIP11 and AtbZIP10 results in substantial differences in up-regulated gene sets when compared to single bZIP expression. Altogether these data suggest a crucial functional impact of bZIP heterodimerisation. 


\title{
3 Members of the bZIP C/S1 network form specific heterodimers
}

\section{Two-hybrid protein-protein interaction analysis in Arabidopsis protoplasts: establishment of a heterodimerization map of group $\mathrm{C}$ and group $\mathrm{S}$ bZIP transcription factors}

\author{
Andrea Ehlert ${ }^{1, \dagger}$, Fridtjof Weltmeier ${ }^{1, \dagger}$, Xuan Wang $^{1}$, Caroline S. Mayer ${ }^{1}$, Sjef Smeekens ${ }^{2}$, Jesús Vicente-Carbajosa ${ }^{3}$ and \\ Wolfgang Dröge-Laser ${ }^{1, *}$ \\ ${ }^{7}$ Albrecht-von-Haller-Institut, Universität Göttingen, Untere Karspüle 2, D-37073 Göttingen, Germany, \\ ${ }^{2}$ Molecular Plant Physiology, University of Utrecht, Padualaan 8, $3584 \mathrm{CH}$ Utrecht, The Netherlands, and \\ ${ }^{3}$ Dept. Bioquimica y Biologia Molecular, ETSI Agronomos, Universidad Politecnica, Ciudad Universitaria, 28040 Madrid, Spain
}

Received 20 December 2005; revised 2 February 2006; accepted 8 February 2006.

"For correspondence (fax +49 (0) 55139 7820; e-mail wdroege@gwdg.de).

'Joint first authors.

\begin{abstract}
Summary
In vivo protein-protein interactions are frequently studied by means of yeast two-hybrid analysis. However, interactions detected in yeast might differ considerably in the plant system. Based on GAL4 DNA-binding (BD) and activation domains (AD) we established an Arabidopsis protoplast two-hybrid (P2H) system. The use of Gateway-compatible vectors enables the high-throughput screening of protein-protein interactions in plant cells. The efficiency of the system was tested by examining the homo- and heterodimerization properties of basic leucine zipper (bZIP) transcription factors. A comprehensive heterodimerization matrix of Arabidopsis thaliana group $\mathrm{C}$ and group $\mathrm{S}$ bZIP transcription factors was generated by comparing the results of yeast and protoplast two-hybrid experiments. Surprisingly, almost no homodimerization but rather specific and selective heterodimerization was detected. Heterodimers were preferentially formed between group C members (AtbZIP9, -10,-25, -63) and members of group S1 (AtbZIP1, -2, -11, -44, -53). In addition, significant but low-affinity interactions were detected inside group S1, S2 or C AtbZIPs, respectively. As a quantitative approach, $\mathrm{P} 2 \mathrm{H}$ identified weak heterodimerization events which were not detected in the yeast system. Thus, in addition to cell biological techniques, $\mathrm{P} 2 \mathrm{H}$ is a valuable tool for studying protein-protein interaction in living plant cells.
\end{abstract}

Keywords: two-hybrid analysis, protein-protein interaction, Arabidopsis thaliana, groups C and S bZIP transcription factors, heterodimerization.

Protein-protein interaction has been considered as increasingly important for the molecular understanding of biological processes (Pawson and Nash, 2003). Several methods have been proposed for studying protein-protein interaction in vitro and in vivo. In particular, yeast two-hybrid approaches have been very successful in identifying putative protein interaction partners (Fields and Song, 1989). However, the data obtained from yeast and plant cells might differ considerably, and therefore, in planta confirmation of the interaction is necessary. Biochemical approaches like coimmunoprecipitation (Co-IP) or cytological methods like fluorescence resonance energy transfer (FRET) (Wallrabe and Periasamy, 2005) or bimolecular fluorescence complementation (BiFC) (Hu and Kerppola, 2003; Huang et al., 2005; Walter et al., 2004) have been used. The latter are of advantage in identifying the cytological localization of a given protein interaction. However, these methods do not quantify the affinity of the protein partners. Furthermore, 
these methods are limited when large numbers of protein partners have to be tested. In order to enable highthroughput screening of protein interactions in planta, an Arabidopsis thaliana protoplast two-hybrid $(\mathrm{P} 2 \mathrm{H})$ assay was established which utilizes Gateway ${ }^{16}$-compatible (Invitrogen, Karlsruhe, Germany) vectors.

To test the efficiency of the $\mathrm{P} 2 \mathrm{H}$ system, dimerization of basic leucine zipper (bZIP) proteins was analysed. The bZIP proteins are ubiquitously distributed transcriptional regulators characterized by a basic DNA-binding domain (Landschulz et al., 1988). In general, bZIP proteins bind DNA as dimers mediated by the so-called leucine zipper domain (ZIP), a heptad repeat of leucine or other bulky hydrophobic amino acids creating an amphipathic helix (Baxevanis and Vinson, 1993; Landschulz et al., 1988). The formation of bZIP homo- or heterodimers offers a tremendous combinatorial flexibility to a regulatory system. The DNA-binding specificity and affinity, transactivation properties and, ultimately, cell physiology might be altered by heterodimerization (Naar et al., 2001). In the model plant $A$. thaliana, 75 bZIP proteins have been identified and classified into 10 groups (Jakoby et al., 2002). In general, bZIP proteins do not heterodimerize promiscuously; specific interactions are preferred (Newman and Keating, 2003). Thus, heterodimerization is anticipated to fulfil an important function in gene regulation. In tobacco (Strathmann et al., 2001) and parsley (Rügner et al., 2001) specific heterodimerization was found among members which are related to the Arabidopsis group $S$ and group C bZIP proteins (Jakoby et al., 2002). The function of group $C$ and group $S$ bZIP proteins has as yet hardly been assessed. These bZIP transcription factors are thought to be involved in important functional aspects such as seed development (Lara et al., 2003), carbohydrate partitioning (Rook et al., 1998) or stress responses (Satoh et al., 2004).

In this work we have compared the dimerization properties of Arabidopsis group $\mathrm{S}$ and group $\mathrm{C}$ bZIPs in yeast and protoplast two-hybrid approaches. A matrix displaying specific interactions has been established. In particular strong heterodimerization was found between members of the groups $\mathrm{C}$ and $\mathrm{S} 1$. Moreover, the $\mathrm{P} 2 \mathrm{H}$ system enables discrimination between weak and strong interactions.

\section{Results}

\section{Arabidopsis group $C$ and group $S$ bZIP transcription factors}

Since specific heterodimerization between group $C$ and group $\mathrm{S}$ bZIP proteins has been described in tobacco (Strathmann et al., 2001) and parsley (Rügner et al., 2001) this finding prompted us to analyse heterodimerization in the model plant Arabidopsis, where the complete bZIP gene family has been described (Jakoby et al., 2002). In Arabidopsis, group $\mathrm{C}$ and group $\mathrm{S}$ consist of four and 17 members, respectively. In comparison with other bZIP proteins, these transcription factors harbour the extraordinarily high number of eight hydrophobic amino acid repeats (Figure 1a, b). Based on homology of the bZIP domains, the 17 members of group $\mathrm{S}$ can be divided into three subgroups, designated $\mathrm{S} 1$, S2 and S3 (Figure 1c). Members of group C differ with respect to their $\mathrm{N}$-terminal and $\mathrm{C}$-terminal domains surrounding the central bZIP domain. Whereas AtbZIP10, AtbZIP25 and AtbZIP63 show high amino acid homology, AtbZIP9 is more distantly related (Figure 1a).

Comprehensive yeast two-hybrid analysis reveals specific bZIP heterodimerization preferentially between groups $C$ and $\mathrm{S} 1$

Due to their conserved bZIP domain, group $\mathrm{C}$ and group $\mathrm{S}$ proteins have been classified as transcription factors. In order to define their capacity in regulating transcription, AtbZIP factors were fused to the DNA-binding domain (BD) of the yeast transcriptional activator GAL4 (Giniger et al., 1985) and expressed in yeast. The ability of BD-AtbZIP fusion proteins to bind GAL4 upstream activating sequences (GALUAS) and to activate the transcription of a lacZ reporter gene was quantified by $\beta$-galactosidase assays (Figure 2). Strong activation capacity was found for the group $C$ members AtbZIP9 and AtbZIP10, which prevents further interaction studies in yeast. AtbZIP53, AtbZIP25 and AtbZIP63 did not show any intrinsic activation properties, whereas the activation capacities of the other AtbZIPs were rather weak.

Transcription factors of the bZIP family bind DNA predominantly as homo- or heterodimers (Cranz et al., 2004; Ellenberger et al., 1992; Metallo and Schepartz, 1997). Making use of a yeast two-hybrid system, heterodimerization between members of groups $\mathrm{C}$ and $\mathrm{S}$ was detected by prototrophic growth of the yeast strains on plates without uracil (Figure 3a). Protein-protein interaction was further elucidated quantitatively by measuring lacZ activity (Figure $3 \mathrm{~b}, \mathrm{c})$. Table 1 summarizes the results, indicating that only selective protein-protein interactions occur. In particular, the group C bZIP proteins heterodimerized with members of subgroup $\mathrm{S} 1$. No interaction was observed between group $\mathrm{C}$ and subgroups S2 or S3. Some weak heterodimerization was detected among members of subgroup S1 (Figure 3c, Table 1). Surprisingly, homodimerization was hardly detected in the yeast two-hybrid system when the prototrophic growth of the yeast colonies was analysed. In agreement with this, quantitative $\beta$-galactosidase assays revealed only very weak homodimerization capacities. For instance, the BD-AtbZIP63/AD-AtbZIP1 heterodimer (AD being the GAL4 DNA-activation domain) produced a $\beta$ galactosidase activity of 22.1 units, whereas homodimerization of AtbZIP63 or AtbZIP1 resulted in an activity of 1.0 and 0.38 units, respectively. From these data we conclude that the heterodimerization of AtbZIPs of subclass $C$ and 
892 Andrea Ehlert et al.

(a)

gabcdef gabcdef gabcdef gabcdef gabcdef gabcdef gabcdef gabcdef gabcdef

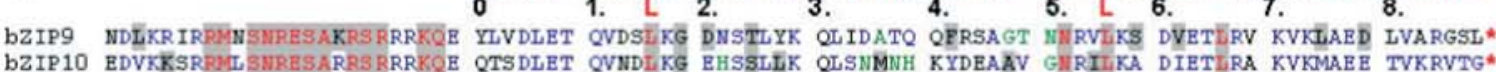

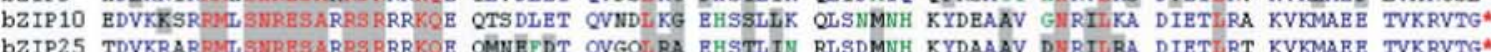
bZIP63 TIVKRVKRNLSIRESARPSRRRKA HLSELET OVSQLRV ENSILMK GLTDVTQ TENDASV EIIRVIKA MIETLRA KVKMAEE TVKRLTGA

(b) gabcdef gabcdef gabcdef gabcdef gabcdef gabcdef gabcdef gabcdef gabcdef

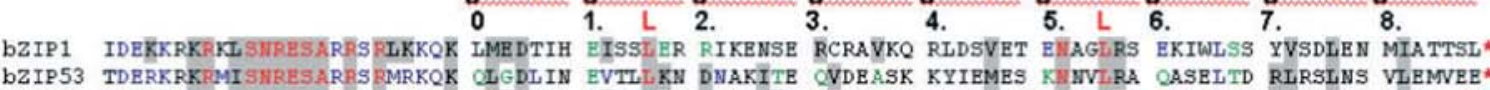
bZIP2 VDERKRKMLSIRESARUSRMRKOK HVDDLTA OINO SH DNRQIIN SLTVTSQ I MMKIOA ENSVITA CMEELST RLQSLNE IVDLVQS* bZIP11 MEQRKRKMU SIRESARPSEMKKOK LLDDLTA OVMH KK ENTEIVT SVSITTQ HYTVEA EIISVIRA OLDELNH RLQSLND IIEFLDS* bZIP44 IDERKRKR KQSMRESARRSSMRKOK HLDDLTA OVTH RK ENAQIVA GIAVTTQ HYVTIEA BIIDI RA OVLELNH RLQSLNE IVDFVES*

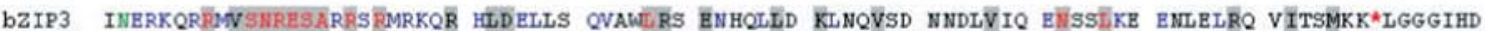

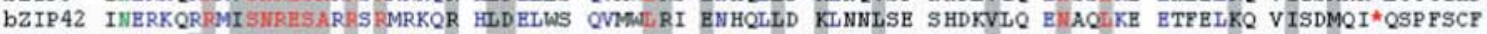

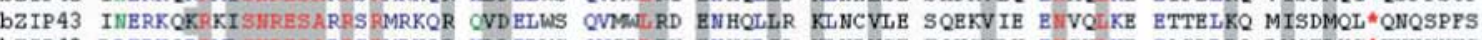

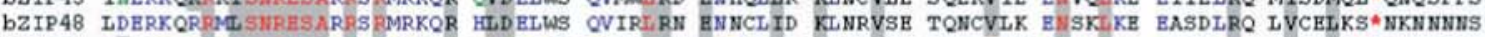
DZIP58 DE-RKQRMISHRESARP STMRKQR HLDELWS QVIRURT DNHCLMD KLNRVSE SHELALK ENAKLKE ETSDLRQ L ISEIKS*HNEDDNS

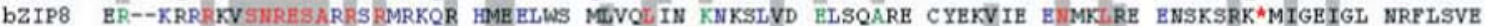

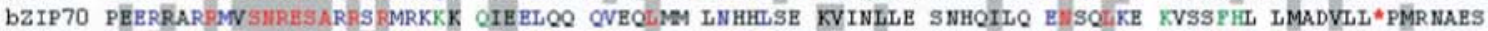

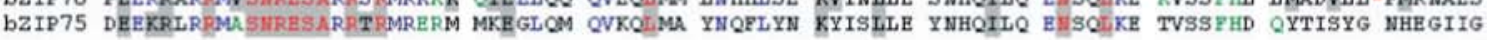

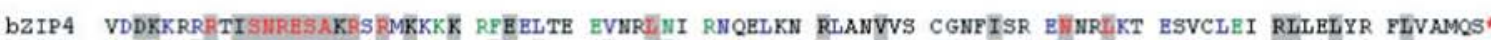
bZIP5 TDERKKKKKL SIIRESAKFS REKKOK HLEEMSI CINOLII ONQELKN OLRYVLY HCQRTKM EIIDRILM EHRILHD KLLNIRQ VLMFRQT

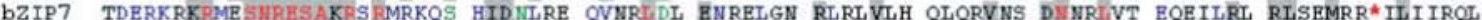

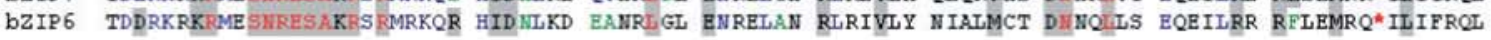

(c)

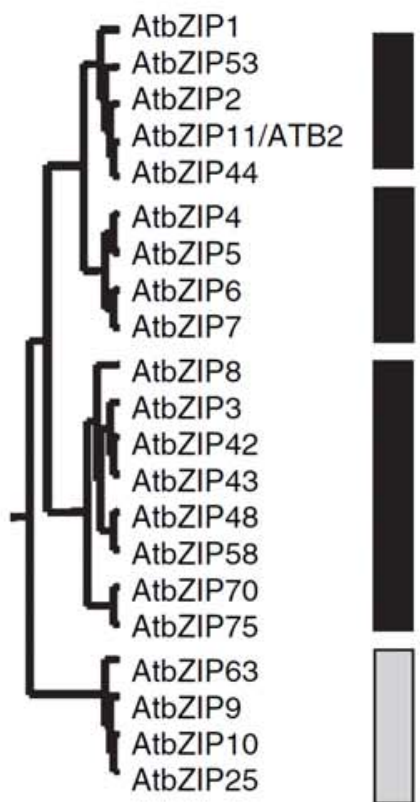

(d)

\section{Group S1}

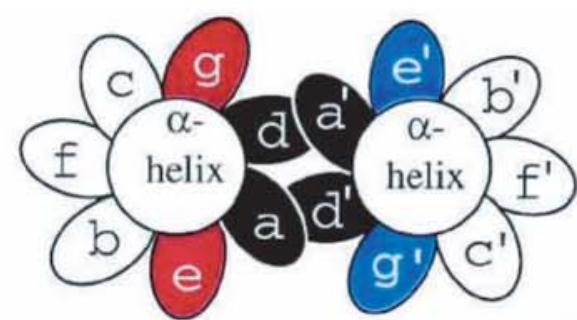

Group S2

Group C

Figure 1. Classification of $A$. thaliana groups $C$ and $S$ bZIP transcription factors (Jakoby et al., 2002)

(a) Amino acid (aa) alignment of the bZIP domains of the group C members AtbZIP9 (At5g24800), AtbZIP10 (At4g02640), AtbZIP25 (At3g54620) and AtbZIP63 (At5g28770).

(b) Amino acid alignment of the bZIP domains of the group S members. Conserved aa are depicted in red The positions of the aa in the heptad repeats of the leucine zipper are labelled with gabcdef (Landschulz et al., 1988). Termination of the $\alpha$-helical ZIP domain as predicted by Deppmann et al. (2004) is indicated (*).

(c) Based on an aa alignment of the bZIP domains, members of group S have been subdivided into the subgroups S1, S2 and S3.

(d) Heterodimerization of two bZIP proteins is mediated by interaction/repulsion of the aa located in positions a and d as well as e and g (Deppmann et al., 2004; Fong et al., 2004).

subclass $\mathrm{S}$ is preferred over homodimerization. Moreover, heterodimerization does not occur promiscuously, but in a very specific manner.
In addition to the formation of $\mathrm{C} / \mathrm{S} 1$ heterodimers, strong interaction between the group S2 members AtbZIP58 and AtbZIP42 was detected (Figure 4). It is important to note that 


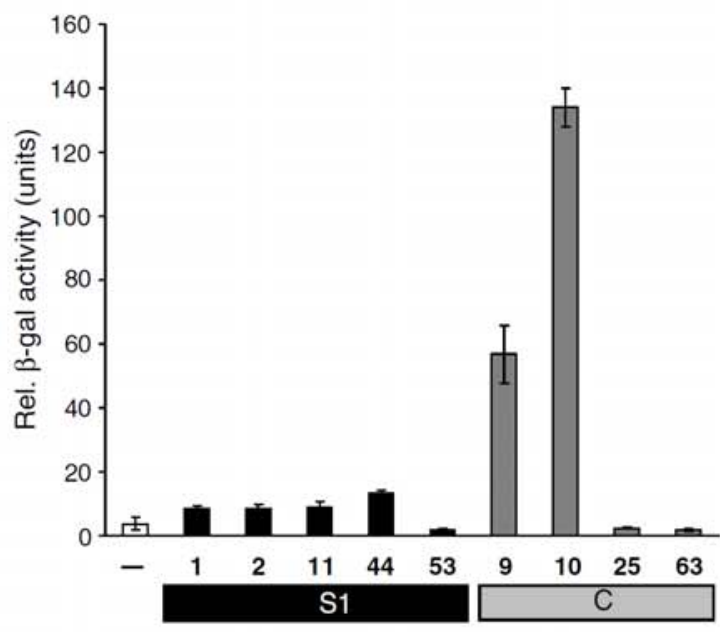

Figure 2. Activation properties of BD-AtbZIP proteins in yeast.

The indicated BD-AtbZIP fusion proteins were expressed in the Proquest yeast two-hybrid strain MaV203. Activation of a lacZ reporter driven by GALUAS elements was measured by quantitative $\beta$-galactosidase ( $\beta$-gal) activity assays. Mean values and standard deviations are calculated from fou measurements obtained from two independent yeast transformants. Data related to group $\mathrm{C}$ and group $\mathrm{S} 1 \mathrm{bZIPs}$ are coded in grey or black, respectively.

AtbZIP42 is the only protein tested which shows reasonable homodimerization activity in yeast (Figure 4a).

\section{A two-hybrid approach to the study of protein-protein} interaction in plant-derived protoplasts

To quantitatively assess protein-protein interaction in planta, the principle of the yeast two-hybrid approach was established for transiently transformed Arabidopsis protoplasts (Figure 5a). In order to enable a highthroughput protein-protein interaction screening, Gateway ${ }^{8}$-compatible high-copy vectors were constructed which allow the expression of $A D$ or $B D$ fusion proteins under the control of the strong $35 \mathrm{~S}$ promoter (Figure $5 \mathrm{~b}$ ). The ATG start codon was optimized (Sprenger-Haussels and Weisshaar, 2000) to ensure a high level of translation in plant cells. The $A D$ and $B D$ constructs harbour nuclear localization sequences (NLS) which target the fusion proteins to the nucleus, where protein interactions are measured by reporter gene activity. In order to test whether given interaction partners exhibit activation properties without the heterologous $A D$ domain, a third vector was constructed which allows the expression of HA (haemagglutinin)-tagged proteins (Figure $5 \mathrm{~b}$ ). Expression of the proteins in the transformed protoplasts can be verified by using commercial antibodies raised against the GAL4 $B D$ and $A D$ domain or the HA tag, respectively (Figure $5 c$ ).

Arabidopsis protoplasts were transfected using the PEG (polyethylenglycol) method described by Sheen (2001). (a)

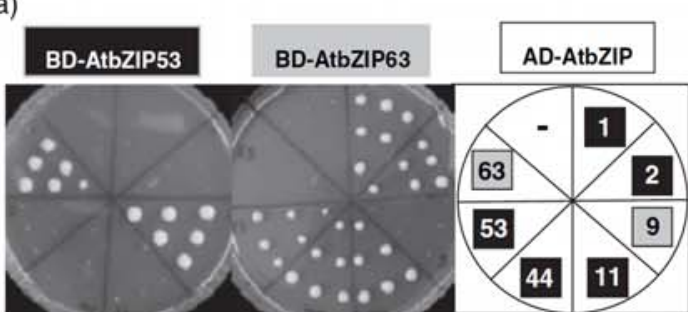

(b)

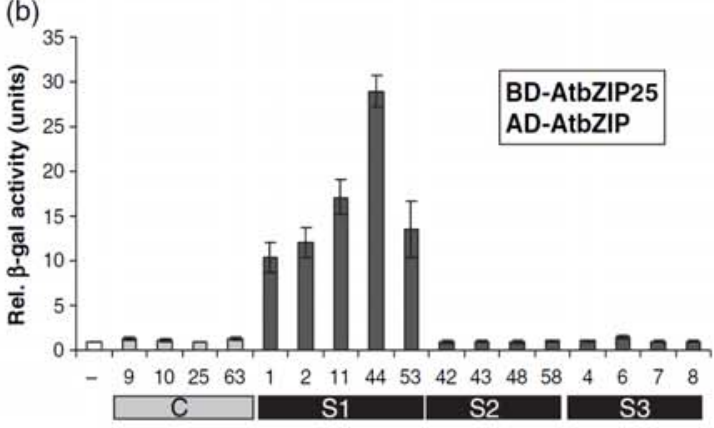

(c)

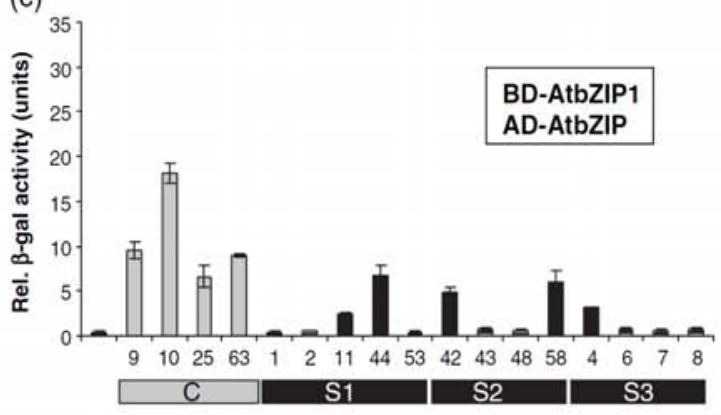

Figure 3. Heterodimerization analysis of groups $\mathrm{C}$ and $\mathrm{S} 1 \mathrm{bZIP}$ protein using a yeast two-hybrid assay.

(a) Prototrophic growth of the yeast two-hybrid strain MaV203 on medis depleted in uracil indicates heterodimerization of the analysed bZIP partners. Co-transformants of BD-AtbZIP53 (left) and BD-bZIP63 (middle) with the ADbZIP proteins indicated on the right.

(b, c) Activation of a lacZ reporter driven by GAL-UAS elements was measured by quantitative $\beta$-galactosidase ( $\beta$-gal) activity assays. We anaysed co-transformants expressing the AD-AtbZIP proteins indicated and BDAtbZIP25 (b) or BD-AtbZIP1 (c). Mean values and standard deviations calculated from four measurements obtained from two independent yeast clones are given. The bZIPs from groups $\mathrm{C}$ and $\mathrm{S}$ are coded in grey or black, respectively.

Variation in the transfection efficiencies was corrected by co-expression of a $\mathrm{Pro}_{35}$ : $\mathrm{NAN}$ (synthetic neuraminidase gene) reporter (Kirby and Kavanagh, 2002), the activity of which can easily be measured in the same buffer system and with the same equipment that is used for GUS assays. Using this procedure, highly reproducible data were obtained and the number of repetitions reduced.

To test the efficiency of the system we analysed group C/ $\mathrm{S} 1$ interactions for AtbZIP which had been observed in the 
894 Andrea Ehlert et al.

Table 1 Interaction pattern of group C and group S AtbZIP transcription factors obtained by yeast two-hybrid analysis

\begin{tabular}{|c|c|c|c|c|c|c|c|c|c|c|c|}
\hline \multirow[b]{3}{*}{ AD-AtbZIP } & \multicolumn{11}{|c|}{ BD-AtbZIP } \\
\hline & \multicolumn{4}{|l|}{ C } & \multicolumn{5}{|l|}{ S1 } & \multicolumn{2}{|l|}{ S2 } \\
\hline & AtbZIP9 & AtbZIP10 & AtbZIP25 & AtbZIP63 & AtbZIP1 & AtbZIP2 & AtbZIP11 & AtbZIP44 & AtbZIP53 & AtbZIP42 & AtbZIP58 \\
\hline \multicolumn{12}{|l|}{ c } \\
\hline AtbZIP9 & A & A & - & - & + & + & + & + & + & - & - \\
\hline AtbZIP10 & A & A & - & - & + & + & + & + & + & - & - \\
\hline AtbZIP25 & A & A & - & - & + & + & + & + & + & - & - \\
\hline AtbZIP63 & A & A & - & - & + & + & + & + & + & - & - \\
\hline \multicolumn{12}{|l|}{$\mathrm{S} 1$} \\
\hline AtbZIP1 & A & A & + & + & - & - & $(+)$ & $(+)$ & - & - & - \\
\hline AtbZIP2 & A & A & + & + & - & - & - & - & - & - & - \\
\hline AtbZIP11 & A & A & + & + & $(+)$ & - & - & - & - & - & - \\
\hline AtbZIP44 & A & A & + & + & + & - & - & - & - & - & - \\
\hline AtbZIP53 & A & A & + & + & - & - & - & - & - & - & - \\
\hline \multicolumn{12}{|c|}{ S2 } \\
\hline AtbZIP42 & A & A & - & - & $(+)$ & - & - & $(+)$ & - & t & + \\
\hline AtbZIP58 & A & A & - & - & + & - & $(+)$ & + & $(+)$ & + & $(+1)$ \\
\hline AtbZIP43 & A & A & - & - & - & - & - & - & - & - & - \\
\hline AtbZIP48 & A & A & - & - & - & - & - & - & - & - & $(+)$ \\
\hline \multicolumn{12}{|l|}{ S3 } \\
\hline AtbZIP4 & A & A & - & - & $(+)$ & - & - & - & - & - & - \\
\hline AtbZIP6 & A & A & - & - & - & - & - & - & - & - & - \\
\hline AtbZIP7 & A & A & - & - & - & - & - & - & - & - & - \\
\hline AtbZIP8 & A & A & - & - & - & - & - & - & - & - & - \\
\hline
\end{tabular}

Bait proteins, BD-AtbZIP; prey proteins, AD-bZIP; -, no interaction/activation; (+), weak interaction $(2-5 \times$ rel. $\beta$-galactosidase units); + , strong interactions ( $>5 \times$ rel. $\beta$-galactosidase units); $A$, no quantification due to autoactivation of the bait; grey background shows homodimers.

yeast system. Intrinsic activation properties of the BD-bZIP fusion protein were measured by co-expression with a

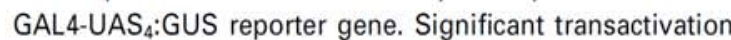
properties were found for AtbZIP2, AtbZIP11 and AtbZIP44, but not for the other members of groups $C$ and $S 1$ (Figure 6a). Activation of the reporter depends on the GALBD-mediated DNA binding. The control experiments in Figure 6(a) clearly demonstrate that AD-AtbZIP proteins cannot bind or activate the GAL-UAS ${ }_{4}: \mathrm{GUS}$ reporter. Hence, the binding of the BD domain to GAL-UAS is specific to plant cells.

Interaction of a BD-AtbZIP protein with a protein partner fused to the $A D$ domain should result in strong activation of the GAL4-UAS 4 :GUS reporter. Figure 6 (b) shows data from typical experiments where the interaction between non-autoactive BD-AtbZIP1 and the other group_S and group $C$ bZIPs were studied. Strong interaction of BDAtbZIP1 was observed in particular with AtbZIP10 and to a lesser extent with AtbZIP25 (Figure 6b). Due to the quantitative read-out, the method allows us to differentiate strong from weak interactions, such as that of AtbZIP1 with AtbZIP11. The use of the group $C$ member AtbZIP25 as a bait again revealed that interactions between groups $\mathrm{C}$ and $\mathrm{S} 1$ are preferred in comparison to heterodimerization within group $\mathrm{S}$ or group $\mathrm{C}$ factors, respectively (Figure 6c).
Establishment of an in planta heterodimerization matrix of group $\mathrm{S} 1$ and group $\mathrm{C}$ bZIPS

Because the yeast two-hybrid analysis revealed specific interactions between all group $\mathrm{C}$ and group S1 bZIPs, the $\mathrm{P} 2 \mathrm{H}$ system was used to establish a comprehensive in planta interaction map (Table 2). In principle, the data observed in yeast could be confirmed with the $\mathrm{P} 2 \mathrm{H}$ system. However, in contrast to the interactions observed in yeast, not all interactions between groups $\mathrm{C}$ and $\mathrm{S} 1$ appear to be as strong as in the plant system (e.g. AtbZIP1/AtbZIP63). Furthermore, in contrast to the yeast system, some additional interactions within the group C AtbZIPs were detectable (e.g. AtbZIP10/ AtbZIP63), indicating that the plant-cell-specific environment influences the protein-protein interaction pattern. In summary, the $\mathrm{P} 2 \mathrm{H}$ approach seems to be a highly sensitive tool for studying protein interactions in living plant cells.

\section{Discussion}

In this study, we establish a Gateway ${ }^{6}$-based two-hybrid assay, which enables quantitative high-throughput studies of protein-protein interactions in plant cells. The feasibility of the system was demonstrated by analysing bZIP heterodimerization in comparison with a classical yeast-based twohybrid system. 
(a)

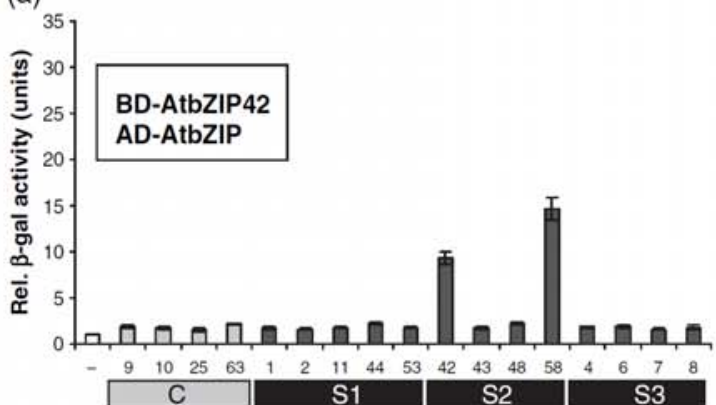

(b)

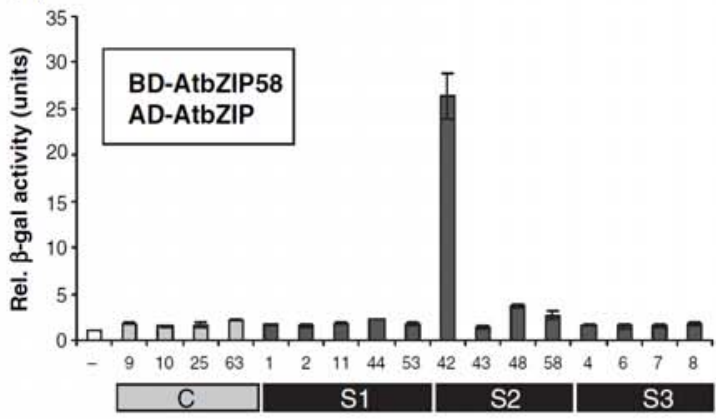

Figure 4. Heterodimerization of group S2 bZIP protein using a yeast twohybrid assay.

Activation of a lacZ reporter driven by GAL-UAS elements was measured by quantitative $\beta$-galactosidase ( $\beta$-gal) activity assays. We analysed the cotransformants expressing the AD-AtbZIP proteins indicated as well as $B D$ AtbZIP42 (a) or BD-AtbZIP58 (b). Mean values and standard deviations calculated from four measurements obtained from two independent yeast clones are given. The bZIPs from groups $\mathrm{C}$ and $\mathrm{S}$ are coded in grey or black, respectively.

Protoplast two-hybrid (P2H) assays enable high-throughput protein-protein interaction studies in plant cells

Yeast two-hybrid screenings are well-established tools for identifying protein interaction partners, particularly those involved in plant signalling processes. However, since

Figure 5. Development of a protoplast based two-hybrid system $(\mathrm{P} 2 \mathrm{H})$ in Arabidopsis.

(a) Set-up of the GAL4-based interaction system using GAL4 activation domain $(A D)$ and GAL4 binding domain (BD) fusions. Interaction of the proteins $X$ and $Y$ is measured using a GAL-UAS ${ }_{4}$ :GUS reporter. For normal ization of transformation efficiency a $\mathrm{PrO}_{35}$ : NAN construct was used (Kirby and Kavanagh, 2002).

(b) The Gateway-compatible (GW) vectors p35S-GAD-GW, p35S-GBD-GW and p35S-HA-GW enable expression of $A D, B D$ or HA tag fusion proteins in protoplasts under control of the $35 \mathrm{~S}$ promoter (Pro35S). CmR, chloramphenicol resistance gene; $A m p R$, ampicillin resistance gene; $c c d B$, constitutive cell death (negative selection marker), attR1/2 GW recombination sites; polyA, polyA site.

c) Immunological detection of group S1 and group C bZIP proteins indicated after expression in Arabidopsis protoplasts. The figure shows Western blots making use of a HA-tag-specific antibody. ", unspecific background staining. cellular conditions might greatly affect protein-protein interaction, it is essential to confirm the findings in a plantcell system. In this work, we transferred the principle of the GAL4-based two-hybrid system (Fields and Song, 1989) to plant cells. Specific binding of BD fusion proteins to the GAL$U_{A S_{4}}:$ GUS reporter was demonstrated, whereas AD fusion

(a)

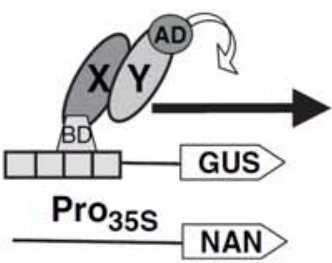

(b)

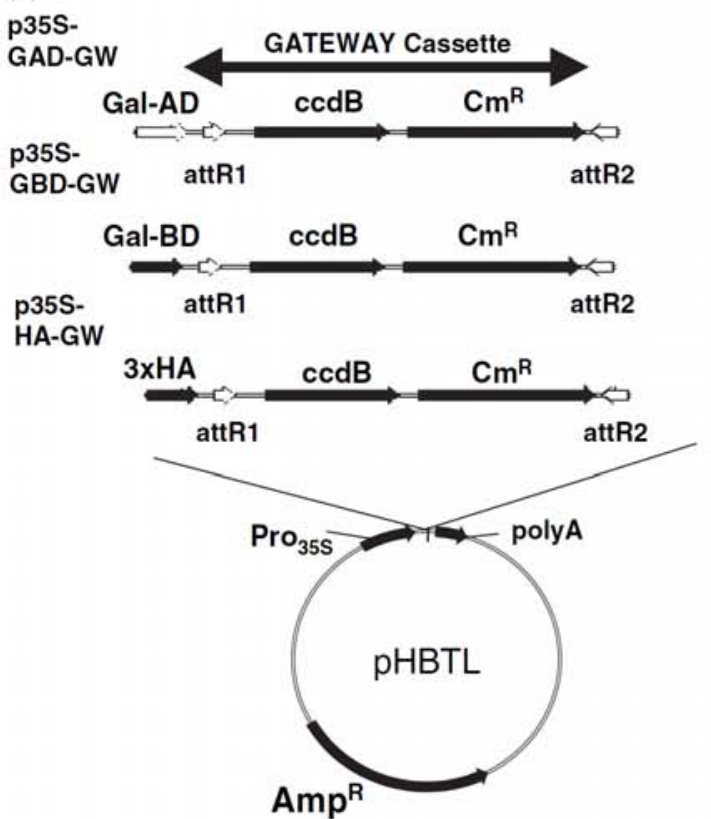

(c)
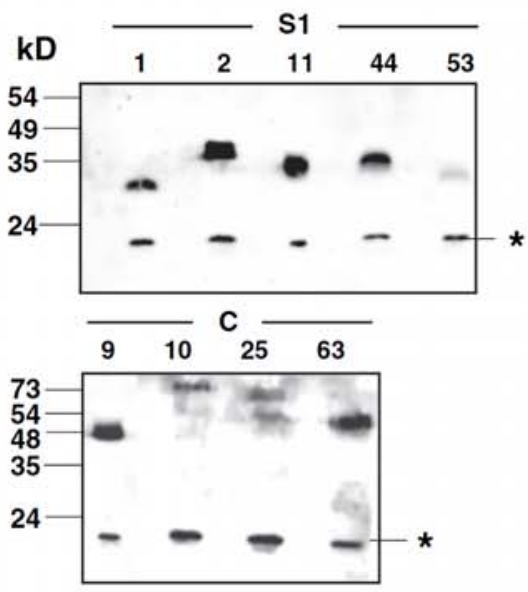

2006 The Authors

Journal compilation @2 2006 Blackwell Publishing Ltd, The Plant Journal, (2006), 46, 890-900 
896 Andrea Ehlert et al.

proteins do not bind and activate the reporter. Protein-protein interaction leads to strong activation of the reporter mediated by the $A D$ domain, which has been found to function in the plant system. In comparison to other approaches, the $\mathrm{P} 2 \mathrm{H}$ system offers a number of advantages:

(a)

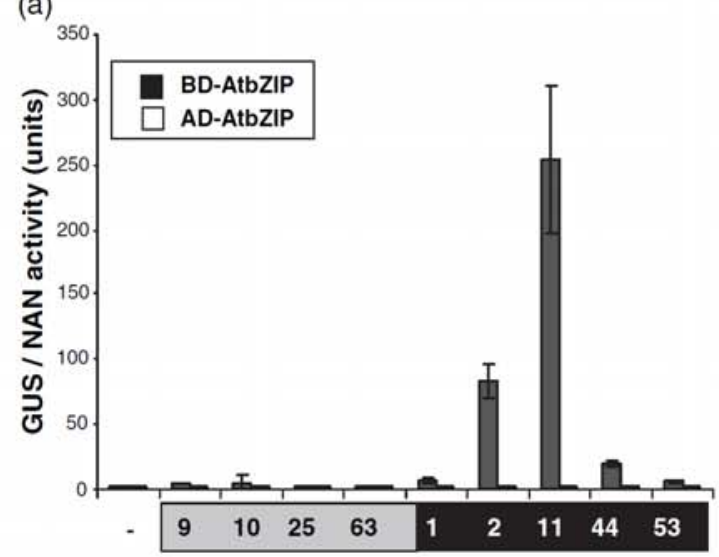

(b)

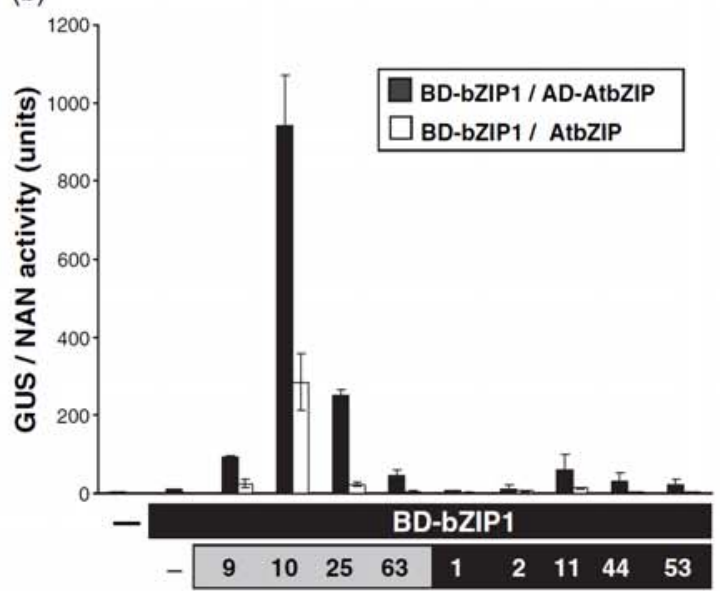

(c)

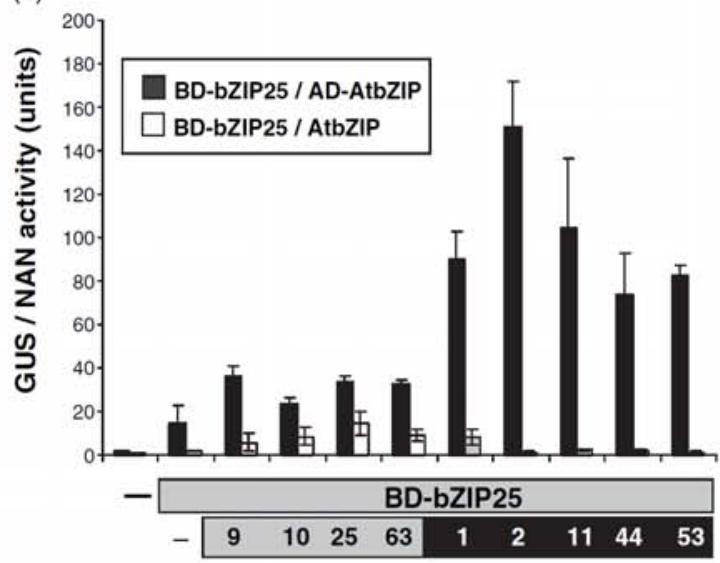

(i) It is an in planta assay system. Comparison of bZIP interactions studied in yeast shows that many but not all the interactions are identical in the plant system. This finding can be explained by plant-specific factors or conditions which might influence interactions in different cellular systems.

(ii) Intrinsic activation properties have been found to be a problem in yeast two-hybrid systems. The study presented here demonstrates that interactions can be observed even if the baits show intrinsic activation properties.

(iii) The protoplast two-hybrid assay is an in vivo assay. In comparison with Co-IP methods, the interactions are studied without disruption of the cellular context under in vivo conditions.

(iv) The protoplast two-hybrid assay is easy to handle using standard plant laboratory equipment and methods. This is particularly true when compared with methods like FRET and BiFC.

(v) By applying Gateway ${ }^{8}$-compatible vectors, $\mathrm{P} 2 \mathrm{H}$ can be used as a high-throughput technology.

(vi) By using a $\mathrm{PrO}_{35}$ s:NAN normalization construct, the GUS and NAN reporter assays can be performed in the same test tube using standard plate reading equipment. In our hands, the assays have been found to be highly reliable and reproducible.

(vii) The use of commercial tag-specific antibodies (HA, AD, $\mathrm{BD}$ ) enables control of the expression levels of the expressed proteins.

(viii) Finally, but most importantly, $\mathrm{P} 2 \mathrm{H}$ allows a quantitative read-out of protein-protein interactions. Whereas FRET and BIFC methods allow cytological localization of protein-protein interactions, the quantification of the fluorescence read-out is difficult.

Like all approaches, $\mathrm{P} 2 \mathrm{H}$ has its limitations and therefore several methods should be used in combination to confirm a protein-protein interaction:

(i) As in the classical yeast two-hybrid analysis, the interaction is studied in the nucleus and therefore might be limited for specific classes of proteins. In particular, the use of $\mathrm{P} 2 \mathrm{H}$ with respect to proteins involved in transcriptional control has been successfully demonstrated.

Figure 6. Heterodimerization of groups $\mathrm{C}$ and $\mathrm{S} 1 \mathrm{bZIP}$ proteins using a $\mathrm{P} 2 \mathrm{H}$ assay system in A. thaliana protoplasts.

(a) Activation of a GAL-UAS :GUS reporter gene was measured after expression of the BD-AtbZIP proteins indicated (black bars). In control experiments, AD-AtbZIP proteins (white bars) do not bind and activate the reporter. Co-expression of BD-AtbZIP1 (b) or BD-AtbZIP25 (c) with the AD-bZIP proteins indicated. Activation of the GAL-UAS:GUS reporter indicates heterodimerization. In every transfection experiment $9 \mu \mathrm{g}$ of reporter and $28 \mu \mathrm{g}$ of effector plasmid DNA was applied. In co-transfections $14 \mu \mathrm{g}$ of each bZIP effector plasmid was used. Variations in transformation efficiency are normalized by using $3 \mu \mathrm{g}$ of a Pro $_{355}:$ NAN plasmid construct per experiment (Kirby and Kavanagh, 2002). Mean values and standard deviations of four independent transfections calculated as relative GUS/NAN activities (units) are given. 
Table 2 Interaction pattern of group $\mathrm{C}$ and group S1 AtbZIP transcription factors obtained by P2H analysis. Activation of a GAL-UAS GUS $_{4}$ reporter was quantified after expression of BD-AtbZIP proteins (-) and co-expression of BD-AtbZIP and AD-AtbZIP fusion proteins. Mean values of $\mathrm{GAL}_{4}$ :GUS reporter gene activity (in relative GUS/NAN units) of four independent transfection experiments and the standard deviation (SD) are given. Grey background shows homodimers

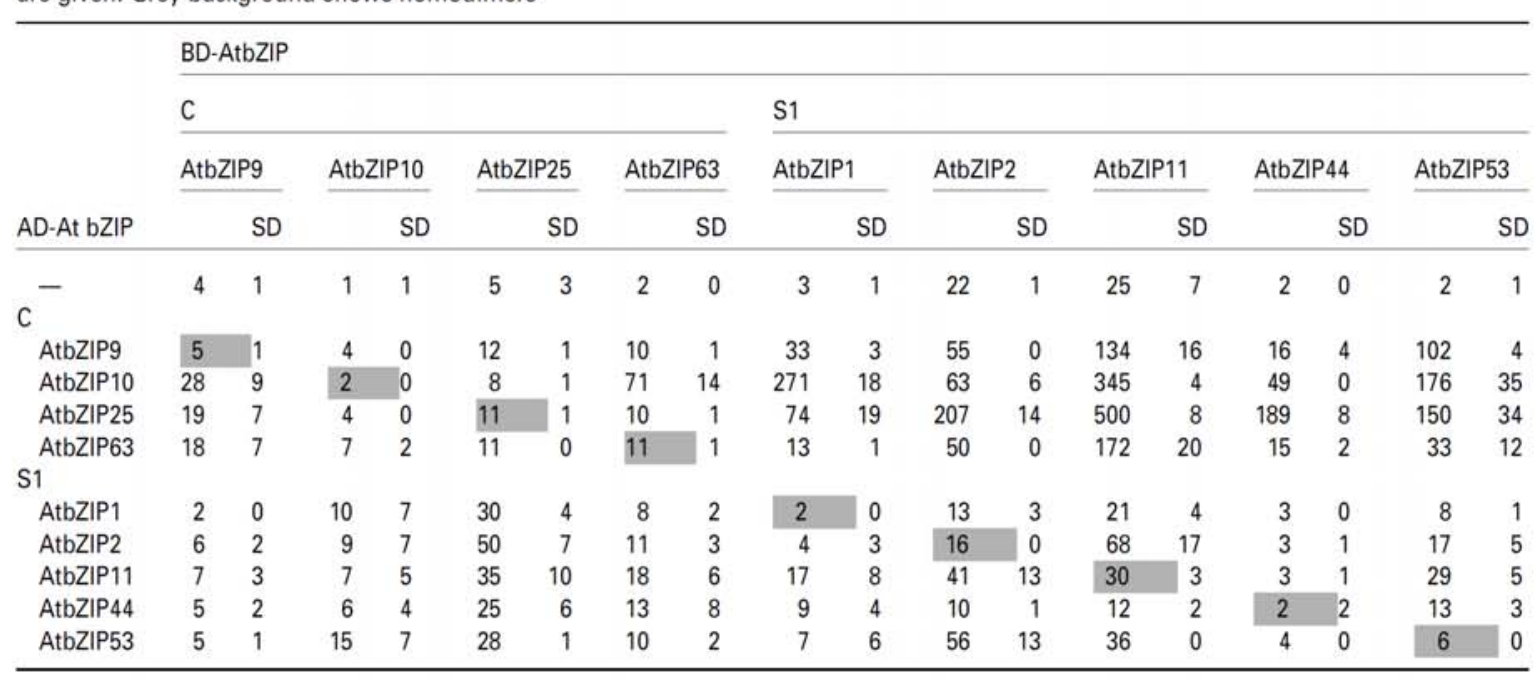

(ii) The reporter gene read-out is based on transcriptional control. Hence, if one of the protein partners or a combination of both partners has an influence on transcription, this would consequently affect the readout. We therefore provide a vector without an $A D$ domain, which can be used to control the reporter gene read-out without activation mediated by the $A D$ domain.

(iii) The detection system is based on the expression of fusion proteins, which might compromise protein function. As has been described for the yeast two-hybrid, BiFC and FRET systems, interaction might not work bidirectionally, depending on whether a $B D$ or an $A D$ domain is fused to the $\mathrm{N}$-terminus or $\mathrm{C}$-terminus of the protein of interest. In the described example of bZIP factors, we found that group $S$ factors tolerate $B D$ fusions, whereas the read-out of all protein interactions of group C BD fusions is much lower (see Table 2). Normalization of the data can be done by statistical means, such as calculating over average values. This would enhance interaction values, for example, of AtbZIP9 and group $\mathrm{C}$ members.

In consequence, although the reporter gene read-out gives clear information on whether protein interaction occurs or not, the quantitative values have to be evaluated with care, as they might not have been entirely related to protein-protein interaction properties of the partners. As in yeast systems, switching between $\mathrm{N}$ - and $\mathrm{C}$-terminal fusions is advisable.

\section{Groups $C$ and $S 1$ bZIP transcription factors form specific heterodimers}

Heterodimerization of bZIP transcription factors has been implicated to be an important mechanism in transcriptional control (Newman and Keating, 2003). However, as in other systems, heterodimerization in Arabidopsis is proposed not to occur promiscuously (Newman and Keating, 2003), but rather in a specific manner (Armstrong et al., 1992; Schindler et al., 1992).

The $\mathrm{P} 2 \mathrm{H}$ system offers the unique possibility of analysing a large number of protein interaction events in parallel. As an example, we studied bZIP heterodimerization. In agreement with our yeast interaction data and with data obtained from highly related bZIP proteins from tobacco (Strathmann et al., 2001) and parsley (Rügner et al., 2001) group C bZIPs heterodimerize preferentially with members of group S1 (Figure 7). These findings could also be confirmed by using in vitro or in vivo techniques, such as far-Western (Strathmann et al., 2001) or BiFC (CSM and WDL, unpublished results). Because no interaction with selected members of the subgroups S2 and S3 or other groups such as group $A$ and group D (AE, FW, WDL, data not shown) resulted in an activation of the reporter gene, we conclude that the bZIP proteins analysed here are highly selective with respect to their heterodimerization properties.

The affinity of the members of groups $\mathrm{C}$ and $\mathrm{S}$ to form homodimers is rather low. This is in contrast to in vitro DNAbinding experiments which show that homodimers of group S (Satoh et al., 2004) or group C (Lara et al., 2003) can bind DNA. This finding could be explained by monomeric DNA binding which has been described before (Cranz et al., 2004; Metallo and Schepartz, 1997). Alternatively, structural analysis of bZIP proteins has demonstrated, that, by DNA binding, the conformation of the basic domain turns into a coiled structure which is linear with the ZIP domain (Pu and Struhl, 1991). Hence, the formation of a DNA/bZIP 
898 Andrea Ehlert et al.

Y2H:

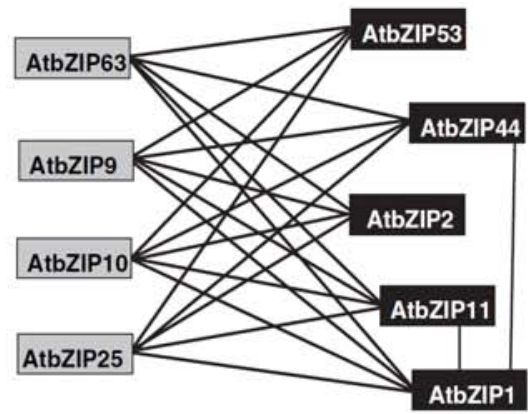

P2H:

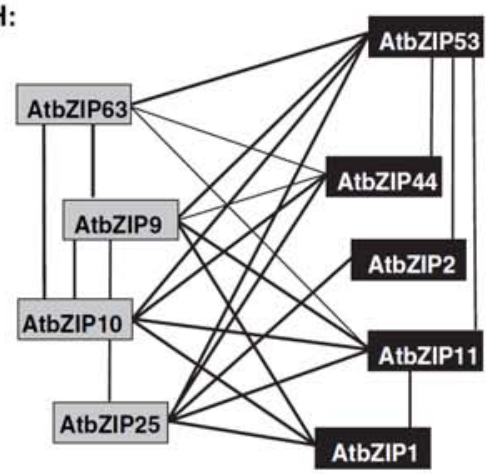

Figure 7. Comparison of the heterodimerization of group C/S1 bZIP transcription factors detected by $\mathrm{Y} 2 \mathrm{H}$ (upper panel) or $\mathrm{P} 2 \mathrm{H}$ (lower panel). Weak interactions and strong heterodimerizations are depicted by thin or solid lines, respectively.

protein complex might alter the dimerization properties. Our experimental system makes use of the GAL4 DNA-binding domain and, hence, protein interaction is measured independent of DNA binding mediated by the basic domain. It is tempting to speculate whether selective dimerization would occur in the absence of target DNA under in vivo conditions and if, therefore, heterodimer formation could be involved in the recognition and guidance of transcription factors to their specific target promoters.

In addition to the high-affinity group $\mathrm{C} / \mathrm{S} 1$ heterodimers, some interactions between members of group S2 (AtbZIP42) AtbZIP58), group C (AtbZIP 10/AtbZIP63) or group S1 (AtbZIP53/AtbZIP11) were also observed. Although these interactions are weak, they can be reproducibly detected by the $\mathrm{P} 2 \mathrm{H}$ system. Genome-wide interaction screens will provide the basis to elucidate the biological relevance of particular heterodimers.

Mutations, which specifically disrupt the $\alpha$-helical structure of the ZIP domain, inhibit interaction, indicating that the protein-protein interaction is mediated by the ZIP domain ( $A E, F W$, WDL unpublished results). Resulting from knowledge obtained in Homo sapiens and Drosophila melano- gaster, Deppmann et al. (2004) postulated a simple model to predict homo- and heterodimerization in Arabidopsis based on (i) the number of heptad (abcdefg) repeats, (ii) the presence of asparagines or charged amino acids in position $\mathrm{a}$, and (iii) the presence of interhelical electrostatic interactions (mainly in positions $\mathrm{g}$ and e) (see Figure 1a,d). However, the experimental data obtained in this work would not have been predicted from these simple rules. A more complicated algorithm was suggested by Fong et al. (2004) and verified by comparison with near-complete protein array data obtained for the human bZIP proteome (Newman and Keating, 2003). This algorithm predicts some, but not all, of the interactions we found in this study, indicating that more experimental data are necessary to acquire full knowledge of the heterodimerization pattern of bZIPs in plant systems.

In contrast to the human bZIP proteins, all proteins of groups $\mathrm{S} 1$ and $\mathrm{C}$ are characterized by an extraordinarily long ZIP domain consisting of eight heptad repeats (Figure 1). Other bZIP proteins, which do not interact with members of groups $C$ and $S 1$, display significantly fewer repeats, such as members of group $D$ or group $A$ (Deppmann et al., 2004). Interestingly, many members of groups S2 and S3 which do not heterodimerize with group C contain proline $(P)$ or multiple glycine $(G)$ residues in their last repeats, which should disrupt the $\alpha$-helical structure resulting in a significantly shortened ZIP domain (e.g. AtbZIP43, AtbZIP3, AtbZIP70; see Figure 1). This would support the idea that these structural features might influence heterodimerization. However, since the five closely related group S1 members show significantly different heterodimerization properties, the number of repeats cannot be the only determinant. Analyses of secondary structure using the PAIRCOIL software (Berger et al., 1995) indicate that proteins with related heterodimerization patterns show a similar conformation, based on their probability of forming $\alpha$-helical structures (data not shown). Furthermore, it is striking that the heptad repeats 6 and 7 of the subgroups S2 and S3 are more distantly related than those of bZIP proteins classified into groups $C$ and S1 (Figure 1). Experimental studies using ZIP-specific mutations will give further insights into how heterodimerization specificity is achieved.

In this work we demonstrated that bZIP heterodimerization does not occur promiscuously but in a specific manner. Protein structure defines heterodimer formation and, ultimately, target gene selection. Hence it can be assumed that heterodimerization plays an important role in gene regulation. The $\mathrm{P} 2 \mathrm{H}$ assay offers a valuable tool for analysing heterodimerization under in planta conditions. These studies are important for defining partners for further functional studies on bZIP transcription factors as well as improving bioinformatic tools for heterodimer prediction. 


\section{Experimental procedures}

\section{Plant material and treatment}

For protoplast preparation A. thaliana ecotype Columbia (Col-O) was grown on soil under controlled environmental conditions in 16h light/8-h dark cycles.

\section{Molecular biological techniques}

Standard DNA techniques have been described in Sambrook et al. (1989). The DNA sequence analyses were performed using an $A B I 310$ sequencer (Applied Biosystems, Darmstadt, Germany) with an $A B I$ PRISM BigDye terminator cycle sequencing reaction kit (Applied Biosystems, Darmstadt, Germany). Western analysis was performed as described in Heinekamp et al. (2002) using a polyclonal HA antibody (Santa Cruz, Santa Cruz, CA, USA).

\section{Vector construction}

For construction of the $\mathrm{P} 2 \mathrm{H}$ vectors, the Gateway ${ }^{\circ}$ cassettes $\mathrm{BD}$ attR1-GW-attR2 and AD-attR1-GW-attR2 were PCR amplified using target DNA from the yeast two-hybrid vectors pDEST32 and pDEST22 (Invitrogen) and the primers $\mathrm{pBD}_{\text {for }}$ (AGGTACCAACAATGAAGCTACTGTCTTCTATCGAAC) and pGW rev (AGGTACCACCACTTTGTACAAGAAAGCTGAA) or $\mathrm{pAD}_{\text {for }}$ (AGGTACCAACAATGCCCAAGAAGAAGCGGAAGGTC) and pGW rev (AGGTACDA CCACTTTGTACAAGAAAGCTGAA), respectively. The translational start (ATG) was optimized (underlined) for plant expression (Sprenger-Haussels and Weisshaar, 2000). The PCR fragments were inserted in the pGEM-T Vector and verified by DNA sequencing. After restriction with Acc651, the GW cassettes were inserted into the protoplast transformation vector pHBT (Sheen, 2001) which enables expression under control of the $\mathrm{PrO}_{355}$.

In order to obtain p35S-HA-GW, the 3xHA-tag-attR1-GW-attR2 cassette located in the plant transformation vector pAlligator2 (Bensmihen et al., 2004) was PCR amplified using the primers AGGTACCAACAATGGCATACCCATACGACGTTCC and AG GTACCACCACTTTGTACAAGAAAGCTGAA. The following steps were performed, as described above.

Gateway entry vectors (pDONR201, pDONR207) containing the different Arabidopsis group $\mathrm{S}$ and group $\mathrm{C}$ bZIP genes were made available by the REGIA project (Jakoby et al., 2002). From the entry vectors the bZIP genes were recombined into the ProQuest $₫$ Y $2 \mathrm{H}$ Vectors pDEST22 and pDEST32 (Invitrogen), p35S-GAD-GW, p35SBD-GW and p35S-HA-GW as described by the manufacturer.

\section{Yeast two-hybrid analysis}

ProQuest ${ }^{8} \mathrm{Y} 2 \mathrm{H}$ vectors pDEST22 and pDEST32 containing the different bZIP genes were transformed into the yeast strain MaV203 (Invitrogen) according to the protocol of Dohmen et al. (1991). Handling of yeast cultures, plate growth assays and quantitative $\beta$ galactosidase assays were performed as described in the Clontech Yeast Protocols Handbook (1996).

\section{Protoplast transformation and GUS assays}

Protoplast isolation and transformation was performed according to the method described by Sheen (2001). For transactivation assays, $9 \mu \mathrm{g}$ of a GAL4-UAS 4 :GUS reporter (B. Weisshaar, University of Bielefeld, Germany) and $14 \mu \mathrm{g}$ of each bZIP effector plasmid were used. For normalization $3 \mu \mathrm{g}$ of Pro $_{35 s}$ :NAN plasmid (Kirby and Kavanagh, 2002) was added. GUS and NAN enzyme assays were performed according to Kirby and Kavanagh (2002). The ratio of GUS and NAN activities are calculated as relative GUS/NAN units using Excel software (Microsoft, Office).

\section{Acknowledgements}

We are grateful to C. Gatz (University of Göttingen, Germany) for support and to F. de Courcy for proofreading. We thank B. Weisshaar (University of Bielefeld, Germany) and T. Kavanagh (University of Dublin, Ireland) for supplying plasmid constructs. This research was supported, in part, by grants from the DFG to AE and FW and the European Community to XW.

\section{References}

Armstrong, G.A., Weisshaar, B. and Hahlbrock, K. (1992) Homodimeric and heterodimeric leucine zipper proteins and nuclear factors from parsley recognize diverse promoter elements with ACGT cores. Plant Cell, 4, 525-537.

Baxevanis, A.D. and Vinson, C.R. (1993) Interactions of coiled coils in transcription factors: where is the specificity? Curr. Opin. Genet. Dev. 3, 278-285.

Bensmihen, S., To, A., Lambert, G., Kroj, T., Giraudat, J. and Parcy, F. (2004) Analysis of an activated ABI5 allele using a new selection method for transgenic Arabidopsis seeds. FEBS Lett. 561, 127-131.

Berger, B., Wilson, D.B., Wolf, E., Tonchev, T., Milla, M. and Kim, P.S. (1995) Predicting coiled coils by use of pairwise residue correlations. Proc. Natl Acad. Sci. USA, 92, 8259-8263.

Cranz, S., Berger, C., Baici, A., Jelesarov, I. and Bosshard, H.R. (2004) Monomeric and dimeric bZIP transcription factor GCN4 bind at the same rate to their target DNA site. Biochemistry, 43, 718-727.

Deppmann, C.D., Acharya, A., Rishi, V., Wobbes, B., Smeekens, S., Taparowsky, E.J. and Vinson, C. (2004) Dimerization specificity of all 67 B-ZIP motifs in Arabidopsis thaliana: a comparison to Homo sapiens B-ZIP motifs. Nucleic Acids Res. 32, 3435-3445.

Dohmen, R.J., Strasser, A.W., Honer, C.B. and Hollenberg, C.P. (1991) An efficient transformation procedure enabling long-term storage of competent cells of various yeast genera. Yeast, 7, 691-692.

Ellenberger, T.E., Brandl, C.J., Struhl, K. and Harrison, S.C. (1992) The GCN4 basic region leucine zipper binds DNA as a dimer of uninterrupted alpha helices: crystal structure of the protein-DNA complex. Cell, 71, 1223-1237.

Fields, S. and Song, O. (1989) A novel genetic system to detect protein-protein interactions. Nature, 340, 245-246.

Fong, J.H., Keating, A.E. and Singh, M. (2004) Predicting specificity in bZIP coiled-coil protein interactions. Genome Biol. 5, R11.

Giniger, E., Varnum, S.M. and Ptashne, M. (1985) Specific DNA binding of GAL4, a positive regulatory protein of yeast. Cell, $\mathbf{4 0}$, 767-774.

Heinekamp, T., Kuhlmann, M., Lenk, A., Strathmann, A. and DrögeLaser, W. (2002) The tobacco bZIP transcription factor BZI-1 binds to G-box elements in the promoters of phenylpropanoid pathway genes in vitro, but it is not involved in their regulation in vivo. Mol. Genet. Genomics, 267, 16-26.

Hu, C.D. and Kerppola, T.K. (2003) Simultaneous visualization of multiple protein interactions in living cells using multicolor fluorescence complementation analysis. Nat. Biotechnol. 21, 539-545.

Huang, T., Bohlenius, H., Eriksson, S., Parcy, F. and Nilsson, $\mathbf{0}$. (2005) The mRNA of the Arabidopsis gene FT moves from leaf to shoot apex and induces flowering. Science, 309, 1694-1696. 


\section{Andrea Ehlert et al.}

Jakoby, M., Weisshaar, B., Dröge-Laser, W., Vicente-Carbajosa, J. Tiedemann, J., Kroj, T. and Parcy, F. (2002) bZIP transcription factors in Arabidopsis. Trends Plant Sci. 7, 106-111.

Kirby, J. and Kavanagh, T.A. (2002) NAN fusions: a synthetic sialidase reporter gene as a sensitive and versatile partner for GUS. Plant J. 32, 391-400.

Landschulz, W.H., Johnson, P.F. and McKnight, S.L. (1988) The leucine zipper: a hypothetical structure common to a new class of DNA binding proteins. Science, 240, 1759-1764.

Lara, P., Onate-Sanchez, L., Abraham, Z., Ferrandiz, C., Diaz, I. Carbonero, P. and Vicente-Carbajosa, J. (2003) Synergistic activation of seed storage protein gene expression in Arabidopsis by $\mathrm{ABI} 3$ and two bZIPS related to OPAQUE2. J. Biol. Chem. 278, 21003-21011.

Metallo, S.J. and Schepartz, A. (1997) Certain bZIP peptides bind DNA sequentially as monomers and dimerize on the DNA. Nat. Struct. Biol. 4, 115-117.

Naar, A.M., Lemon, B.D. and Tjian, R. (2001) Transcriptional coactivator complexes. Annu. Rev. Biochem. 70, 475-501.

Newman, J.R. and Keating, A.E. (2003) Comprehensive identification of human bZIP interactions with coiled-coil arrays. Science, $300,2097-2101$

Pawson, T. and Nash, P. (2003) Assembly of cell regulatory sys tems through protein interaction domains. Science, 300, 445452.

Pu, W.T. and Struhl, K. (1991) The leucine zipper symmetrically positions the adjacent basic regions for specific DNA binding. Proc. Natl Acad. Sci. USA, 88, 6901-6905.

Rook, F., Gerrits, N., Kortstee, A., van Kampen, M., Borrias, M., Weisbeek, P. and Smeekens, S. (1998) Sucrose-specific signalling represses translation of the Arabidopsis ATB2 bZIP transcription factor gene. Plant J. 15, 253-263.
Rügner, A., Frohnmeyer, H., Nake, C., Wellmer, F., Kircher, S. Schäfer, E. and Harter, K. (2001) Isolation and characterization of four novel parsley proteins that interact with the transcriptional regulators CPRF1 and CPRF2. Mol. Genet. Genomics, 265, 964976.

Sambrook, J., Fritsch, E.F. and Maniatis, T. (1989) Molecular Cloning: A Laboratory Manual, 2nd edn. Cold Spring Harbor, NY: Cold Spring Harbor Laboratory Press.

Satoh, R., Fujita, Y., Nakashima, K., Shinozaki, K. and YamaguchiShinozaki, K. (2004) A novel subgroup of bZIP proteins functions as transcriptional activators in hypoosmolarity-responsive expression of the ProDH gene in Arabidopsis. Plant Cell Physiol. 45, 309-317.

Schindler, U., Menkens, A.E., Beckmann, H., Ecker, J.R. and Cashmore, A.R. (1992) Heterodimerization between light-regulated and ubiquitously expressed Arabidopsis GBF bZIP proteins. EMBO J. 11, 1261-1273.

Sheen, J. (2001) Signal transduction in maize and Arabidopsis mesophyll protoplasts. Plant Physiol. 127, 1466-1475.

Sprenger-Haussels, M. and Weisshaar, B. (2000) Transactivation properties of parsley proline-rich bZIP transcription factors. Plant J. 22, 1-8.

Strathmann, A., Kuhlmann, M., Heinekamp, T. and Dröge-Laser, W. (2001) BZI-1 specifically heterodimerises with the tobacco bZIP transcription factors $\mathrm{BZI}-2, \mathrm{BZI}-3 / \mathrm{TBZF}$ and $\mathrm{BZI}-4$, and is functionally involved in flower development. Plant J. 28, 397-408.

Wallrabe, H. and Periasamy, A. (2005) Imaging protein molecules using FRET and FLIM microscopy. Curr. Opin. Biotechnol. 16, 1927.

Walter, M., Chaban, C., Schütze, K. et al. (2004) Visualization of protein interactions in living plant cells using bimolecular fluorescence complementation. Plant J. 40, 428-438. 


\section{Members of the C/S1 bZIP network show overlapping expression pattern}

\section{Expression patterns within the Arabidopsis C/S1 bZIP transcription factor network: availability of heterodimerization partners controls gene expression during stress response and development}

Fridtjof Weltmeier · Fatima Rahmani - Andrea Ehlert · Katrin Dietrich · Katia Schütze • Xuan Wang · Christina Chaban · Johannes Hanson - Markus Teige - Klaus Harter ·

Jesus Vicente-Carbajosa $\cdot$ Sjef Smeekens $\cdot$ Wolfgang Dröge-Laser

Received: 8 April 2008/Accepted: 22 September 2008/Published online: 8 October 2008

(C) The Author(s) 2008. This article is published with open access at Springerlink.com

Abstract Members of the Arabidopsis group C/S1 basic leucine zipper (bZIP) transcription factor (TF) network are proposed to implement transcriptional reprogramming of plant growth in response to energy deprivation and environmental stresses. The four group $\mathrm{C}$ and five group $\mathrm{S} 1$ members form specific heterodimers and are, therefore, considered to cooperate functionally. For example, the interplay of C/S1 bZIP TFs in regulating seed maturation genes was analyzed by expression studies and target gene

Electronic supplementary material The online version of this article (doi:10.1007/s11103-008-9410-9) contains supplementary material, which is available to authorized users.

F. Weltmeier - A. Ehlert - K. Dietrich - X. Wang W. Dröge-Laser $(\square)$

Albrecht-von-Haller-Institut, Universität Göttingen, Untere Karspüle 2. D-37073 Göttingen, Germany

e-mail: wdroege@gwdg.de

F. Rahmani $\cdot$ J. Hanson $\cdot$ S. Smeekens

Molecular Plant Physiology, Utrecht University,

Padualaan 8, Utrecht, $\mathrm{CH} 3584$. The Netherlands

K. Schütze - C, Chaban - K. Harter

Zentrum für Molekularbiologie der Pflanzen,

Pflanzenphysiologie, Auf der Morgenstelle 1,

72076 Tübingen, Germany

M. Teige

Department of Biochemistry, Max F. Perutz Laboratories, University of Vienna, Dr. Bohrgasse 9/5, A-1030 Vienna, Austria

J. Vicente-Carbajosa

Centro de Biotecnología y Genómica de plantas. Departamento

Biotecnología, ETSI Agrónomos, Universidad Politécnica de

Madrid, Avda. Complutense s/n, 28040 Madrid, Spain regulation in both protoplasts and transgenic plants. The abundance of the heterodimerization partners significantly affects target gene transcription. Therefore, a detailed analysis of the developmental and stress related expression patterns was performed by comparing promoter: GUS and transcription data. The idea that the C/S1 network plays a role in the allocation of nutrients is supported by the defined and partially overlapping expression patterns in sink leaves, seeds and anthers. Accordingly, metabolic signals strongly affect bZIP expression on the transcriptional and/or post-transcriptional level. Sucrose induced repression of translation (SIRT) was demonstrated for all group S1 bZIPs. In particular, transcription of group S1 genes strongly responds to various abiotic stresses, such as salt (AtbZIP1) or cold (AtbZIP44). In summary, heterodimerization and expression data provide a basic framework to further determine the functional impact of the C/S1 network in regulating the plant energy balance and nutrient allocation.

Keywords Group C and S1 bZIP transcription factors . Expression analysis · Nutrient allocation .

Energy homeostasis

Introduction

Due to their phototrophic life style, plants continuously adjust their metabolism to day-night rhythms as well as environmental changes, which result in transient energy deprivation. Recently, the Arabidopsis thaliana SnRK-like kinases (sucrose non-fermenting 1(SNF1)-related protein kinases) KIN10 and KIN11 were proposed to function as central signaling regulators mediating adaptation to energy deprivation as well as other stresses (Baena-Gonzalez et al. 
2007). Importantly, these kinases show structural similarities to SNF1-kinase (sucrose non-fermenting 1) in yeast and AMPK (AMP-dependent protein kinase) in mammals which are considered to function as master regulators of the energy balance essential for survival under stress (Polge and Thomas 2007). In plants, the asparagine synthetase gene $(A S N I)$ has been proposed to be a target of the KIN10/11 pathway (Baena-Gonzalez et al. 2007). In Arabidopsis, ASN1 controls the level of the major amino acid asparagine (Lam et al. 1994, 1998, 2003). In comparison to glutamine, asparagine contains more nitrogen than carbon and is, therefore, used to store and transport nitrogen, especially under stress conditions such as prolonged darkness, when carbon is limiting. The transcriptional regulation of dark- or stress-induced $A S N I$ activation is mediated specifically by a G-box cis-element (BaenaGonzalez et al. 2007; Hanson et al. 2008), typically recognized by basic leucine zipper (bZIP) transcription factors (TFs). bZIP proteins are exclusively found in eukaryotic cells and bind DNA by forming homo- or heterodimers (Landschulz et al. 1988). In the Arabidopsis genome, 75 bZIP genes have been identified and classified into 10 groups, referred to as groups A to U (Jakoby et al. 2002). Interestingly, only a specific subset of G-box binding bZIP factors were shown to carry out at least some of the KIN10/ 11 responses (Baena-Gonzalez et al. 2007) namely, AtbZIP2 (GBF5, At2g18160), AtbZIP11 (also named ATB2, At4g34590), AtbZIP53 (At3g62420), and AtbZIP1 (At5g49450). The closely related S1 factor AtbZIP44 (Atlg75390) was not tested but probably participates in these responses as well (Ehlert et al. 2006). In an independent approach, we also identified $A S N 1$ as a target of AtbZIP11 by a micro array analysis of plants expressing AtbZIP11 in an inducible manner (Hanson et al. 2008).

The exact function of group S1 bZIPs is poorly understood. Besides sequence similarity, group S1 bZIPs are characterized by an unusually long $5^{\prime}$ leader containing highly conserved upstream open-reading frames (uORFs). The AtbZIP11 uORFs have been shown to mediate a sucrose induced repression of translation (SIRT) at posttranscriptional level (Wiese et al. 2004), indicating a function in sugar signaling. Accordingly, AtbZIP11, a S1 group member that is up-regulated by light in tissues surrounding the vasculature in carbohydrate-consuming (i.e., sink) tissues, is proposed to be involved in balancing carbohydrate demand and supply (Rook et al. 1998a, 1998b). Recently, AtbZIP53 was shown to be involved in abiotic stress response, regulating proline dehydrogenase expression $(P r o D H)$ during the hypoosmolarity response (Weltmeier et al. 2006). Homologs of the Arabidopsis group S1 bZIPs are present in all the plant species studied and are transcriptionally activated by biotic (Lee et al. 2002) or abiotic stresses such as cold (Shimizu et al. 2005), wounding (Stankovic et al. 2000), drought (Ito et al. 1999), and salt (Kusano et al. 1995). In summary, the currently available data support the postulated function of group S1 bZIPs in regulating energy starvation and other stress responses.

Group S1 bZIPs have been shown to efficiently form specific heterodimers with bZIP members of group $\mathrm{C}$ in planta (Ehlert et al. 2006), namely AtbZIP9 (At5g24800), AtbZIP10 (At4g02640), AtbZIP25 (At3g54620) and AtbZIP63 (At5g28770). So far, few publications have focused on the biological function of group C bZIPs. AtbZIP10 was shown to be involved in oxidative stress response, particularly during defense against the biotrophic pathogen Hyaloperonospora parasitica (Kaminaka et al. 2006). Furthermore, AtbZIP10 and AtbZIP25 were proposed to function as orthologous proteins of the maize OPAQUE2 bZIP factor, which participates in the regulation of seed storage protein (SSP) genes (Lara et al. 2003). SSP synthesis and accumulation strongly depends on resources provided by the "source" tissues to the developing seed, classified as a typical "sink". We have recently demonstrated that heterodimers between group S1 AtbZIP53 and the group C bZIPs, AtbZIP10 or AtbZIP25, are crucial for the full level expression of SSP genes (Alonso et al. submitted).

Heterodimerization between groups $\mathrm{C}$ and $\mathrm{S} 1$ has so far been studied in the context of their role as the regulators of ProDH gene transcription, which was shown to be a direct target of group $\mathrm{C}$ and group S1 heterodimers (Weltmeier et al. 2006). Importantly, heterodimerization results in the strong activation of target genes which cannot be evoked by homodimeric bZIP TFs. Hence, heterodimerization between group $\mathrm{S} 1$ and group $\mathrm{C}$ bZIPs provides an efficient mechanism to enhance transcription of target genes (HIT, heterodimer induced transactivation). As a starting point of a systems biology approach, heterodimerization of all group $\mathrm{S} 1$ and $\mathrm{C}$ were studied in yeast and plant cells (Ehlert et al. 2006) confirming a high affinity for C/S1 interactions. In summary, these findings support the working hypothesis that a complex heterodimerization network of four group $\mathrm{C}$ and five group S1 bZIPs provides a signal integration system operating in plant transcriptional networks.

Here we present a series of examples where the analysis of expression patterns of S1/C bZIPs allows the postulation of functionalities on the basis of potential combinatorial interactions. We provide evidence, by transient expression experiments in protoplasts and transgenic gain-of-function plants, for the function of the $\mathrm{C} / \mathrm{S} 1$ network in regulating gene expression in late seed development. The transcription of target genes is characterized by the coordinated action of pairs of heterodimerizing TFs. Since co-localization of the bZIP partners in the same cell and also in the same cellular 
compartment is a prerequisite for heterodimerization and consequently gene activation, it is essential to know the temporal and spatial expression patterns of the individual AtbZIP TF genes. Transcriptional analysis and studies performed with promoter: GUS lines defined the partially overlapping expression patterns of the bZIP genes confined to typical sink tissues such as seeds or anthers. The regulation by stress stimuli and metabolic signals, such as sugars, was studied at transcriptional and post-transcriptional levels. In summary, we provide a basic data set to dissect this complex regulatory network involved in the plant's energy balance.

\section{Materials and methods}

Plant material and plant transformation

Arabidopsis thaliana ecotype Columbia (Col-O) was grown on soil under controlled environmental conditions at $16 \mathrm{~h}$ light $/ 8 \mathrm{~h}$ dark cycles. To improve germination uniformity, plants were pre-treated at $4^{\circ} \mathrm{C}$ for $2-4$ days. Floral dip transformation was performed by using Agrobacterium tumefaciens strains GVG3101 and wild-type A. thaliana Col-0 (Weigel and Glazebrook 2002). Protoplast transformation was described in Ehlert et al. (2006).

\section{Molecular biological techniques}

Standard DNA techniques have been described in Sambrook et al. (1989). DNA sequence analysis was performed using an $\mathrm{ABI} 310$ sequencer and utilizing an ABI PRISM BigDye Terminator Cycle Sequencing Reaction Kit. Plant RNA was isolated using TRIZOL Reagent (Life Technologies, Rockville, MD, USA). Hybridisation probes were produced by PCR amplification from cDNA using specifically designed primers (Supplementary Table 1). For $2 S 2$ and CRU3 500 bp EcoRI fragments were obtained from the clones pGEM-2S2 and pGEM-CRU3, respectively (Lara et al. 2003). Protoplast isolation and transformation was performed as described in Ehlert et al. (2006).

\section{Vector construction}

GATEWAY $^{\circledast}$ entry vectors (Invitrogen) for all full-length bZIP cDNAs have been described in Ehlert et al. (2006). For ectopic expression, the cDNAs were recombined into the GATEWAY ${ }^{\circledast}$ destination vectors pAlligator2 (Bensmihen et al. 2004).

Vectors encoding Pro AtbZIPII $_{\text {GUS, }}$ Pro $_{\text {AtbZIP53: }}$ GUS, Pro $_{A t b Z I P 2}: G U S$, Pro AtbZIP $10: G U S$ and Pro AtbZIP25:GUS fusions have already been described in Rook et al. (1998a), Wiese et al. (2004) and Weltmeier et al. (2006), respec-

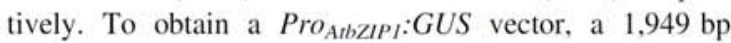
fragment downstream of the AtbZIP1 transcription start site was PCR amplified using the primers given in Supplementary Table 1. SalI and EcoRI restriction sites were added at the $5^{\prime}$ and $3^{\prime}$ of the primer to facilitate directional cloning into the vector pCAMBIA-1391Z (http://www. cambia.org/). The Pro Arbzip44:GUS construct was cloned using standard methods and the promoter sequence fused to the GUS gene of pCAMBIA-1391Z. The 2,784 bp promoter sequence includes the genomic sequence from the SpeI site (2,206 bp upstream of the transcriptional start site) to the EarI site positioned at position 578 of the mRNA encoding region (within the AtbZIP44 protein encoding sequence). DNA fragments of $c a .600 \mathrm{bp}$ from the promoter regions of AtbZIP63 and AtbZIP9 genes were amplified with primers designed with appropriate restriction sites (Supplementary Table 1) and cloned into pBI101 at SalI-BamHI (AtbZIP63) or Sall-XbaI (AtbZIP9) sites to

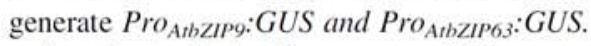

In order to obtain Pro ${ }_{U B O}:$ leader: $G U S$ vectors, sequences of AtbZIP1 (460 bp), AtbZIP2 (456 bp), AtbZIP11 (475 bp), AtbZIP44 (504 bp) and AtbZIP53 (494 bp) were amplified using the primers indicated (Supplementary Table 1). PCR fragments were cloned into the pGEM-TEasy vector (Promega, Madison, USA) and sequenced. Leader fragments were then fused to the GUS-NOS vector pPA2 (http://www.pgreen.ac.uk), using standard restriction/ligation techniques (between Pst//NcoI for AtbZIP53 and between $E c o$ RI/NcoI for the other four). The leader: GUS-NOS fusion fragments were moved to the pGreen0299 vector (EcoRI/HindIII digest) (http://www. pgreen.ac.uk). The $U B Q 10$ promoter was amplified by PCR. The promoter fragment was inserted into the pGreen0229 plasmids containing the leader: GUS fusions (Not $\mathrm{l} / \mathrm{PstI}_{\mathrm{I}}$ restriction). The integrity of the final vectors was confirmed by sequencing.

\section{GUS Assays}

For GUS histochemistry, plant material was stained in a $1 \mathrm{mM}$ 5-bromo-4-chloro-3-indolyl B-D-glucuronide (X-gluc; Biosynth $\mathrm{AG}$ ) solution in $50 \mathrm{mM}$ sodium phosphate, $\mathrm{pH} 7.0,0.1 \%$ Triton $\mathrm{X}-100,1 \mathrm{mM} \mathrm{K}_{3} \mathrm{Fe}(\mathrm{CN})_{6}$, $1 \mathrm{mM} \mathrm{K}_{4} \mathrm{Fe}(\mathrm{CN})_{6}$, at $37^{\circ} \mathrm{C}$ over night. Clearing was in a series of $70 \%$ ethanol. Expression analysis was based on 3-6 independent transgenic lines for each bZIP gene. These lines were selected from 10 to 15 transgenic lines showing significant expression. Quantitative GUS enzyme activity measurements were performed according to Weigel and Glazebrook (2002). 
Results and discussion

Co-localization studies define the function of specific group $\mathrm{C}$ and $\mathrm{S} 1$ members in seed development

Publicly available array data on the expression of group S1 and $\mathrm{C}$ genes during seed development are summarized in Fig. 1a. Interestingly, AtbZIP53 is significantly induced during the late stages of seed development indicating a regulatory function during seed maturation. This expression pattern was confirmed by GUS staining of the

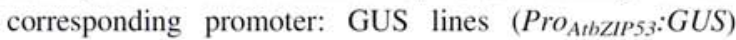
(Fig. 1b) and by in situ hybridization (Alonso et al. submitted). However, as demonstrated in transiently transformed protoplasts, AtbZIP53 was a weak activator of the $2 S$ albumin (2S2) SSP promoter, a typical member of seed maturation genes (Fig. 2a). Remarkably, $2 S 2$ transcription was efficiently activated by AtbZIP53 when

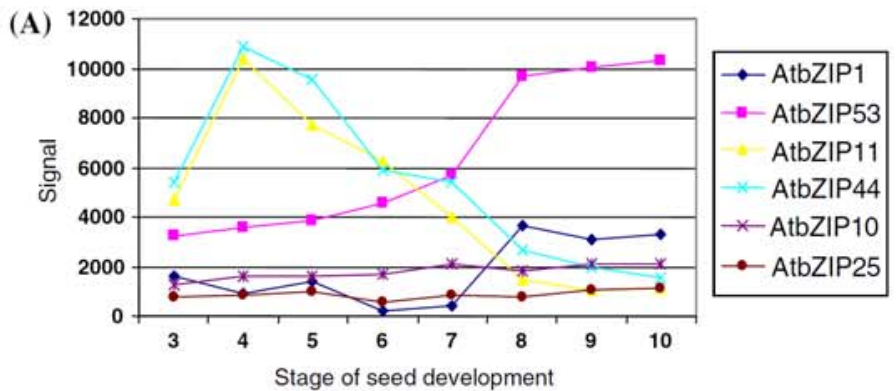

(B)

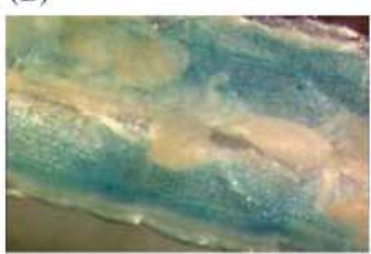

AtbZIP1

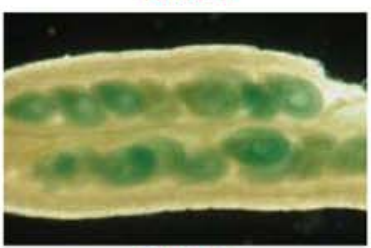

AtbZIP44

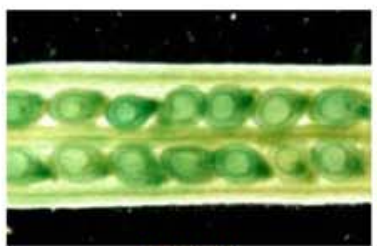

AtbZIP10

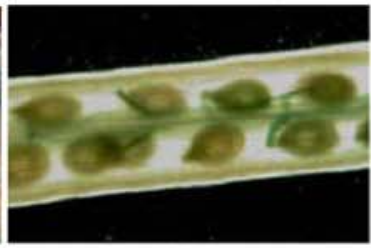

AtbZIP2

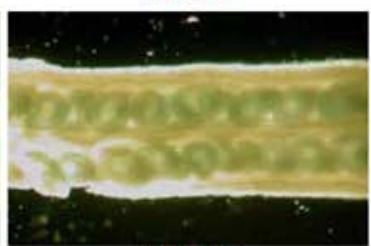

AtbZIP53

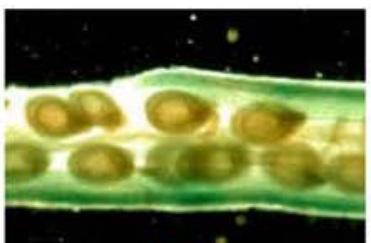

AtbZIP25

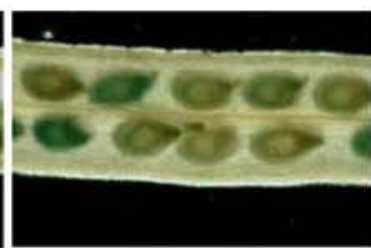

AtbZIP11 / ATB2

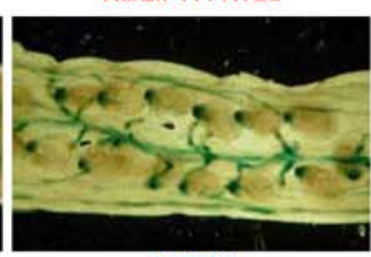

AtbZIP9

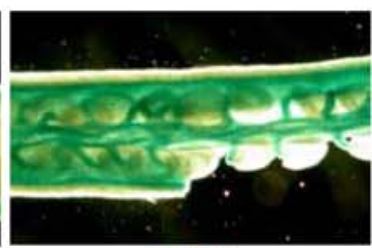

AtbZIP63
Fig. 1 Expression of the group $\mathrm{C}$ and group S1 bZIP genes during seed development. (a) Kinetics of transcript abundance of group C and group S1 AtbZIP genes is based on expression profiles provided by Schmid et al. (2005). Material has been harvested as defined in the following stages of seed development (for details see: http://www. genomforschung.uni-bielefeld.de/GF-research/AtGenExpress-seeds Siliques.html) (3) siliques containing seeds, mid globular to early heart stage (48-66 h after flowering. haf); (4) siliques containing seeds, early heart to late heart (66-84 haf); (5) siliques containing seeds, late heart to mid torpedo (84-90 haf): (6) seeds, mid torpedo to late torpedo ( $90-96$ haf); (7) seeds, late torpedo to early walking-stick
(96-108 haf): (8) seeds, walking-stick to early curled cotyledons (108-120 haf); (9) seeds, curled cotyledons to early green cotyledons (120-144 haf); (10) seeds, green cotyledons (144-192 haf). For each time point the average value of three biological replicates is shown. (b) Histochemical analyzes of GUS activity in young siliques (stage 8-9) of Arabidopsis plants transformed with the indicated Pro Ath. zip:GUS constructs. Plants were grown for 8 weeks on soil under long-day conditions and siliques were harvested $8 \mathrm{~h}$ after beginning of the light phase and stained with $1 \mathrm{mM} \mathrm{X}$-Gluc. For each construct one representative silique is shown. Parts of the siliques are removed to document staining of the seeds 
co-expressed with any group $\mathrm{C}$ heterodimerization partner (Fig. 2b), indicating that co-expression of the group C TFs during late stages of seed development is critical for the proposed function of the bZIPs in regulation of seed maturation genes. Accordingly, in Pro AtbzIPIO GUS lines, the whole carpel showed GUS activity, which became localized to the seeds during development (Fig. 1b). In contrast, only weak activity in seeds and silique valves was
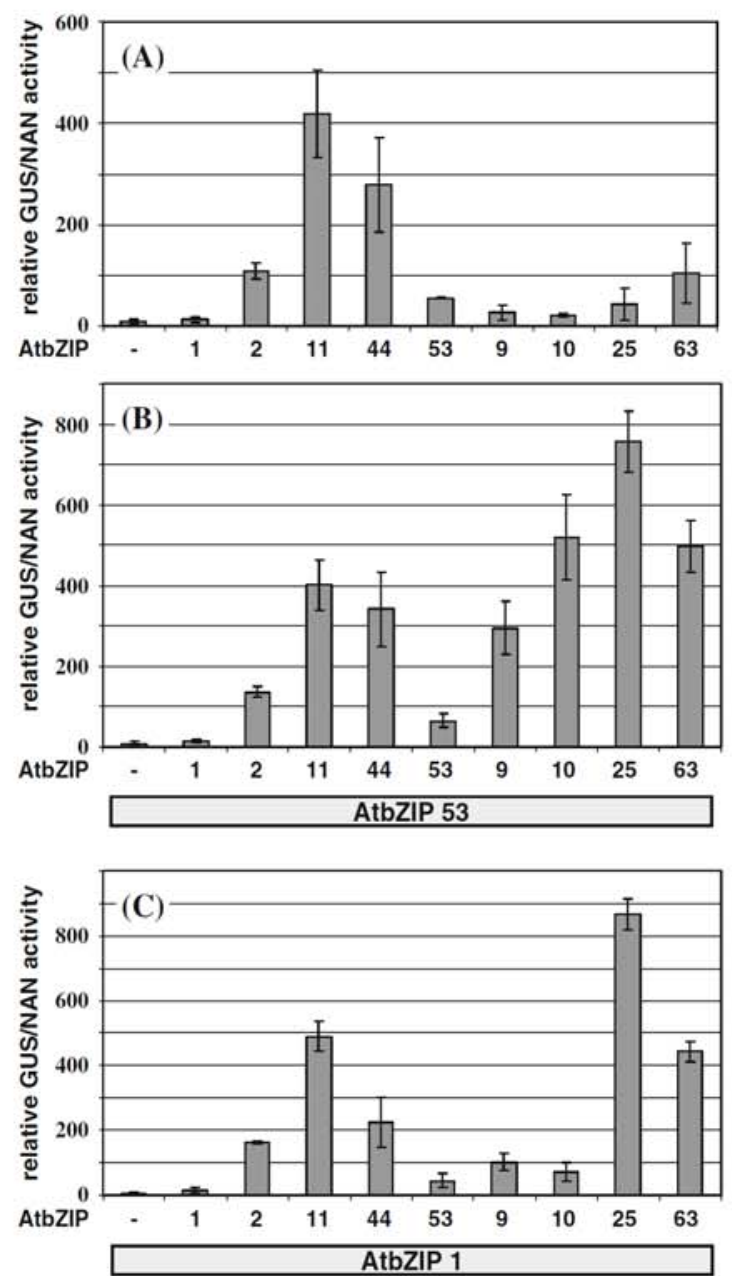

Fig. 2 Regulation of the $2 S$ albumin (2S2) seed storage protein promoter by group $\mathrm{C}$ and group $\mathrm{S} 1$ bZIP TFs in transiently transformed Arabidopsis leaf mesophyll protoplasts. (a) Transfection experiments of a $\mathrm{PrO}_{252}$ : GUS reporter with the group S1 (AtbZIP 1, 2 , 11, 44, 53) or group C (AtbZIP 9, 10, 25, 63) bZIP constructs indicated. (b) Co-transfection of AtbZIP53 with the group S1 and C AtbZIPs indicated. (c) Co-transfection of AtbZIP1 with the group S1 and C AtbZIPs indicated. Given are mean values and standard deviations of four transfections. Amount of DNA used in a reaction: effector plasmid: $14 \mu \mathrm{g}$; reporter plasmid: $9 \mu \mathrm{g}$. The GUS enzyme activity was calculated relative to expression of a NAN standard ( $3 \mu \mathrm{g}$ per reaction) (Ehlert et al. 2006) detectable in Pro ${ }_{\text {ArbZIP25: }}$ GUS lines. The other group C factors showed no GUS activity in seeds, but in the silique valves, vasculature and funiculi, as demonstrated for Pro $_{\text {AtbZIP63: }}$ GUS lines or in vasculature and funiculi, as found in Pro $_{\text {Atbzlpg: }}$ :GUS lines. Thus, although potentially several C/S1 bZIP heterodimers can activate $2 S 2$ target gene transcription, the in planta function is essentially confined by co-expression and co-localization of different bZIP partners.

As reflected in the AtGenExpress data set (Fig. 1a), only the expression of AtbZIP1, the closest homologue of AtbZIP53 (Ehlert et al. 2006), is transcriptionally activated in the late stages of seed development, thus fulfilling the requirements for a regulator of seed maturation genes. As demonstrated in protoplast assays, AtbZIPI shared activation and heterodimerization functions to some extent comparable to those of AtbZIP53. However, 2S2 activation by AtbZIP1/AtbZIP10 was less pronounced when compared to AtbZIP53/AtbZIP10 (Fig. 2c). Accordingly, histochemical analysis of $\mathrm{Pro}_{A t b Z I P I}$ :GUS plants revealed staining of the silique valves but not the seeds (Fig. Ib). Therefore, expression data as well as activation properties suggest that AtbZIPI and AtbZIP53 are functionally different in planta.

AtbZIP2, AtbZIP11 and AtbZIP44 Pro:GUS fusions lead to staining during early seed development (Fig. 1b, Supplementary Fig. 1) (Rook et al. 1998a, b). GUS activity appeared after fertilization, and disappeared when seeds started to mature. Transient GUS activity was primarily observed in the upper ovules and in funiculi, but staining disappeared upon seed maturation, except in the funiculi. These GUS patterns are in accordance with the micro array data sets presented in Fig. Ia. Thus, the specific high expression of AtbZIP11 and AtbZIP44 in early stages of seed development suggests yet unknown functions in embryogenesis but not in seed maturation. Although AtbZIP11 and AtbZIP44 showed a very strong capacity to activate $2 S 2$ transcription in protoplasts (Fig. 2), expression profiles argued against a function in regulating SSP genes.

These findings demonstrate that the transcription factors of the C/S1 network partly overlap with respect to their transcription factor properties but clearly differ in function, due to specific expression and heterodimerization patterns. Further analysis of multiple loss-of-function lines will disclose whether bZIPs are functionally redundant.

Target gene expression is controlled by the cellular abundance of bZIP heterodimerization partners

The amount of the bZIP partners present in individual cells significantly influences target gene expression. As depicted in Fig. 3, the ectopic expression of AtbZIP53, AtbZIP63 or 


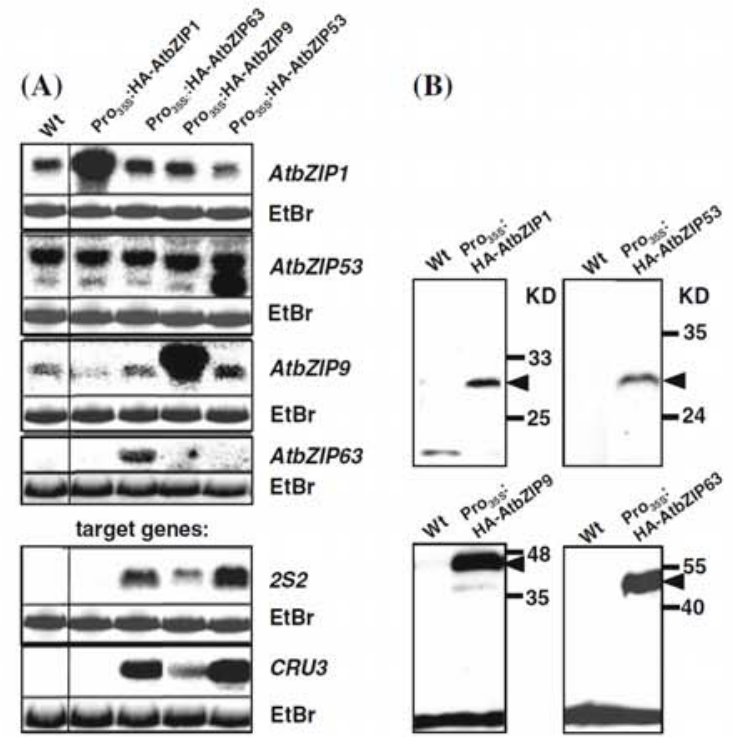

Fig. 3 High abundance of particular group $\mathrm{C}$ and group SI bZIP TFs leads to misexpression of seed storage protein target genes in Arabidopsis seedlings. (a) Activation of $2 S 2$ and cruciferin (CRU3) seed storage protein genes were analyzed in plants ectopically expressing HA tagged derivatives of AtbZIP1. AtbZIP53, AtbZIP63 and AtbZIP9 driven by the $35 \mathrm{~S}$ promoter $\left(\right.$ Pro $\left._{355}\right)$. Northern analyzes of seedlings of the transgenic overexpressors were performed using the radio-labeled probes indicated. Equal loading was confirmed by ethidium bromide (EtBr) staining. (b) Western analysis confirmed expression of the HA tagged bZIP proteins (arrows) HA-AtbZIP1, HA-AtbZIP53, HA-AtbZIP9 and HA-AtbZIP63, respectively. The transgenic plants indicated (right) were compared with wild-type (Wt) plants (left)

AtbZIP9 under the control of the $35 \mathrm{~S}$ promoter $\left(\mathrm{Pro}_{35 \mathrm{~S}}\right)$ led to $2 S 2$ transcription in seedlings, where this gene is normally not expressed. These results demonstrate that AtbZIP63 and AtbZIP9 possess the capacity to activate $2 S 2$ transcription but that their tissue-specific expression pattern limits their in planta function. Manipulating the protein amount of the bZIP genes might shift the balance of bZIP dimers and, consequently, result in the misexpression of target genes. At this point it is not entirely clear whether this ectopic activation of SSP genes is mediated by homodimers formed because of unusually high protein concentrations or whether heterodimers with bZIPs present under these conditions lead to this misexpression of target genes. Importantly, ectopic expression of AtbZIP1 did not activate $2 S 2$ transcription, suggesting that it has a separate function in seed maturation compared to AtbZIP53.

In summary, AtbZIP53 and AtbZIP10 are the most important bZIP TFs for the regulation of SSP gene expression. However, other group C and S1 bZIPs share partly overlapping transactivation, heterodimerization and/ or expression properties. In order to dissect their functions, we performed a comprehensive comparison of expression profiles.

Group C and S1 bZIP factors show distinct expression patterns during anther development

Seed formation and pollen development share many aspects in their physiological status typical of sink tissues. Besides nutrient allocation from source tissues, pollen maturation triggers desiccation tolerance programmes comparable to those existing in the seed to survive harsh environmental conditions. It is well known that overlapping sets of genes are up-regulated during seed and pollen development (Zakharov et al. 2004). For instance, the ProDH gene, encoding a proline degrading enzyme, is strongly activated during Arabidopsis seed and pollen development (Satoh et al. 2002) and the ProDH promoter is a direct target of AtbZIP53/AtbZIP10 during the hypoosmolarity response (Weltmeier et al. 2006). Possibly, the group $\mathrm{C}$ and group $\mathrm{S} 1$ genes are also expressed in other sink tissues of the plant including pollen. The analysis of GUS activity in flowers shortly before pollination revealed two interestingly distinct patterns in the anthers. While the anthers of Pro $_{A t b Z I P l}: G U S$, Pro $_{A t b Z I P 2}: G U S$,

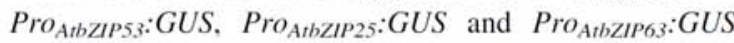
lines showed expression in pollen, GUS activity in

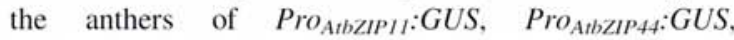

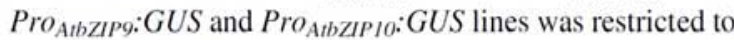
the connective tissue at pre- and post-dehiscence stages and also occasionally in the vascular tissue of the stamen filament vasculature. In the latter group, staining of pollen was never observed (Fig. 4). The strong expression of AtbZIP1 and AtbZIP25 in pollen was confirmed by the high transcript abundance displayed in public array data (Zimmermann et al. 2004). With the exception of AtbZIP10, all group C and S1 bZIP genes showed significant transcript level in stamen, but no detectable transcripts in pollen (Zimmermann et al. 2004). These data further support the assumption that the C/S1 network is involved in the allocation of nutrients to sink organs.

In vegetative tissues group $\mathrm{C}$ and $\mathrm{S} 1$ bZIP genes show distinct expression patterns in sink leaves and the vasculature of stems

To obtain deeper insight into the potential role of the $\mathrm{C} / \mathrm{S} 1$ TF network in green tissues, we extended our expression analysis to 20-day-old green plants at growth stage 20 (Boyes et al. 2001). As shown in Fig. 5, the GUS staining of the plants revealed overlapping but distinct expression

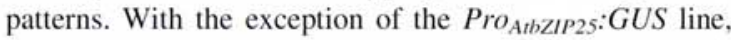
all Promoter:GUS fusions led to staining of young, developing leaves which are considered sink under these 
Fig. 4 Histochemical analyzes of GUS activity in flowers at the time of dehiscence in the

Arabidopsis group S1 (red) and group C (blue) Pro AtbzIP:GUS lines indicated. Plants were grown for 8 weeks on soil under long-day conditions and flowers were harvested $8 \mathrm{~h}$ after beginning of the light phase. For each construct one

representative flower is shown. Interestingly, anthers show GUS activity either in pollen (AtbZIP1, AtbZIP2, AtbZIP53, AtbZIP25, AtbZIP63) or in the connectivum (AtbZIP11. AtbZIP44, AtbZIP9, AtbZIPI0), respectively. Inlet figures are showing representative anthers at higher magnification

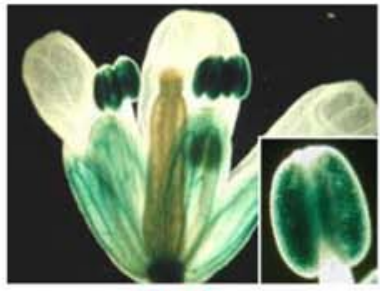

AtbZIP1

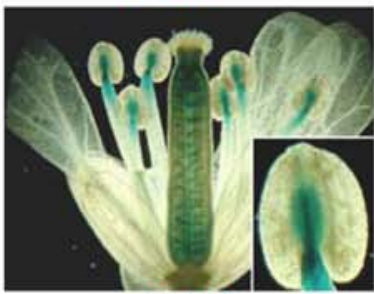

AtbZIP44

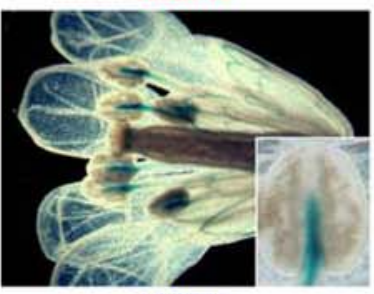

AtbZIP10

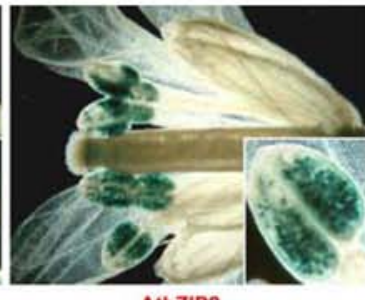

AtbZIP2

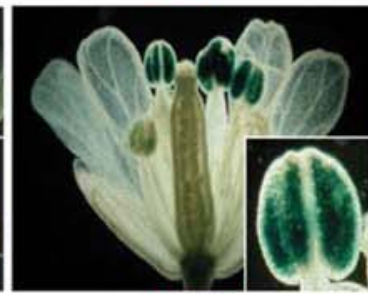

AtbZIP53

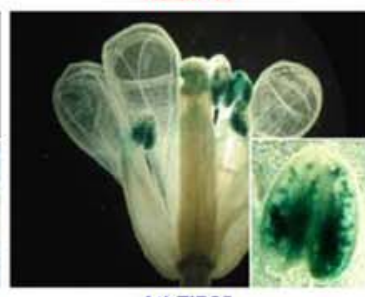

AtbZIP25

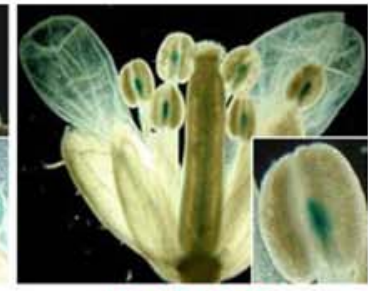

AtbZIP11 / ATB2

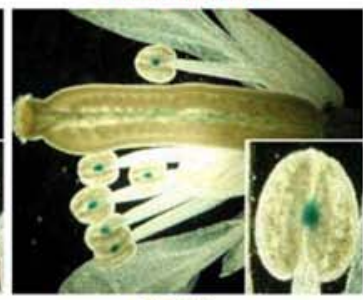

Atbzipg

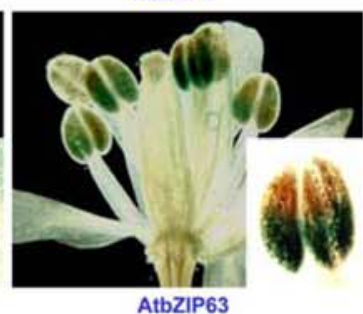

growth conditions. In contrast, the older leaves and cotyledons showed no or much weaker staining. The staining in the young leaves was strongest in the vascular tissue. Compared to the other fusion lines, the staining of Pro AthZIPIO:GUS and Pro AtbZIP44:GUS lines was confined more to the vascular tissue and the activity of the Pro $_{\text {AtbzIPg: }}$ GUS lines was restricted to the vascular tissue only (Fig. 5 and 6). Figure 7 represents an overview, which summarizes GUS results in comparison to the public micro array data sets (Zimmermann et al. 2004). Contradictory to the results from the Pro:GUS analysis, mRNA of bZIP genes were equally abundant in juvenile and adult leaves. However, this finding might be explained by the juvenile leaves used in the mRNA profiling experiment being considerably older than the strongly stained leaves shown in Fig. 5. In particular, for the group S1 Pro AtbzIP:GUS lines, this difference could be explained by post-transcriptional regulation (Wiese et al. 2004) since SIRT could reduce GUS activity in well supplied adult source leaves even at high transcript levels.

AtbZIP10 and AtbZIP25 Pro:GUS lines showed weak

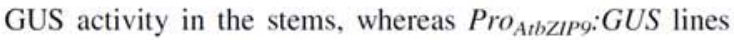
displayed constant staining strictly confined to the phloem (Fig. 6 a-c). In contrast, Pro AtbzIPI:GUS lines showed GUS activity in both the phloem and the xylem (Fig. $6 \mathrm{~d}$ ). Similar GUS activity in the phloem and the xylem was reported for Pro $_{\text {AtbZIPII }}$ :GUS lines and a function of AtbZIP11 in "source-sink" control proposed (Rook et al. 1998a). Altogether, these data support the idea that the $\mathrm{C} / \mathrm{S} 1$ network regulates allocation of nutrients in response to the energy status of the cell (Baena-Gonzalez et al. 2007).

Despite this general similarity, a detailed observation revealed considerable differences in GUS activity. All group S1 Pro:GUS lines showed an intense staining of the stipulate, which, within group $\mathrm{C}$, was generally observed

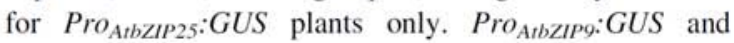
Pro AtbZIP25: GUS also showed no activity in the trichomes, which is regularly observed in the other GUS lines (Fig. 7a). Furthermore, whereas all group S1 Pro:GUS lines often showed pronounced staining in the hydathodes, this is not the case for the group $\mathrm{C}$ members with the exception of the Pro AtbzIPIO:GUS line (Fig. 7a).

We also analyzed GUS activity in roots of 20-day-old

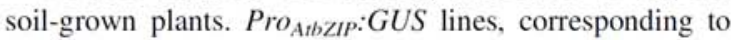
AtbZIP1, AtbZIP2, AtbZIP11 and AtbZIP9, showed an intense staining, which is strongest in, or even restricted to, the vascular tissue (data not shown). AtbZIP44, AtbZIP53, and AtbZIP25 displayed a weaker staining, which, in the Pro $_{\text {AtbZIP53: }}$ GUS line, was variable depending on the experiment. Generally, staining in the root increased with the age of the plant. 
Fig. 5 Histochemical analyzes of GUS activity in 20 days old transgenic Arabidopsis plants expressing Pro Ptwzip: GUS

fusions. Plants were grown on soil under long-day conditions and harvested $8 \mathrm{~h}$ after

beginning of the light phase. For each construct one

representative staining is shown. In Pro $_{\text {AtbzlP25: GUS lines GUS }}$ activity was found only in stipulate, which are shown in magnification next to the whole plant. All plants have been stained with $1 \mathrm{mM} \mathrm{X}$-Gluc except the Pro AtbzlP25:GUS and Pro $_{A t h Z I P 5_{3}: G U S \text { expressing }}$ plants. Because of low GUS activity, $2 \mathrm{mM} \mathrm{X}$-Gluc staining solution has been applied
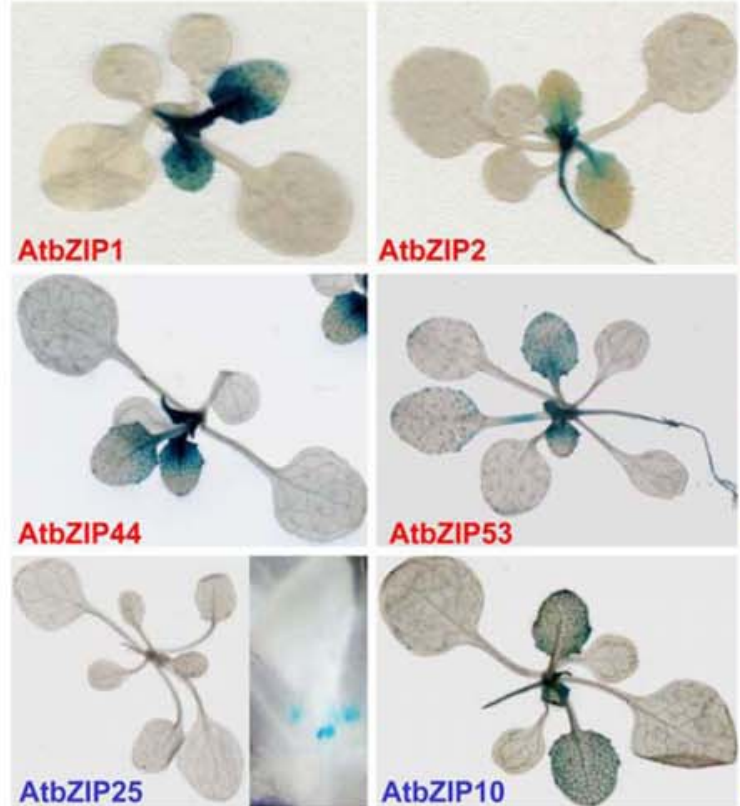

AtbZIP9
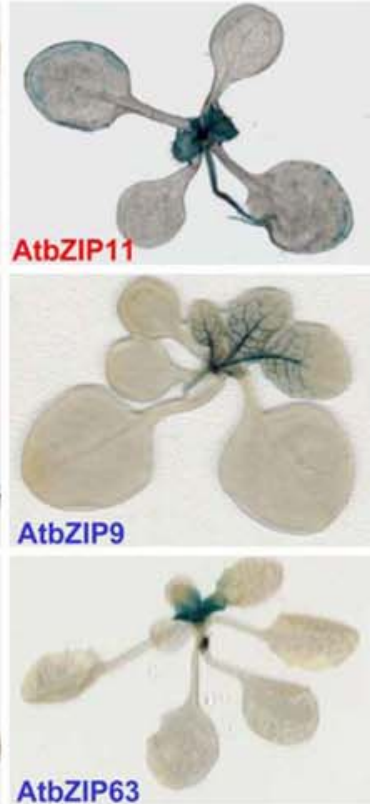

Fig. 6 Detailed analysis of specific Pro $_{\text {Atbzip: }}$ GUS expression patterns.

Pro Atbzlpo:GUS and

Pro distinct tissue-specific GUS activities not found in any other group $\mathrm{C}$ and group $\mathrm{S} 1$ Pro:GUS line. Thin sections of X-Gluc stained leaf (a), root (b) or stem (c) of a Pro Arthzp: GUS line showing phloem-specific activity $(\mathrm{P})$, (d), sections of $X$-Gluc stained stem of a

Pro $_{\text {AtbZIPI: }}$ GUS line showing

GUS activity in phloem $(\mathrm{P})$ as well as in xylem $(\mathrm{X})$

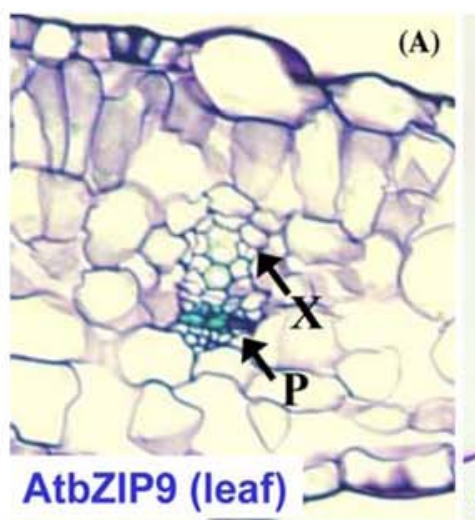

(A)

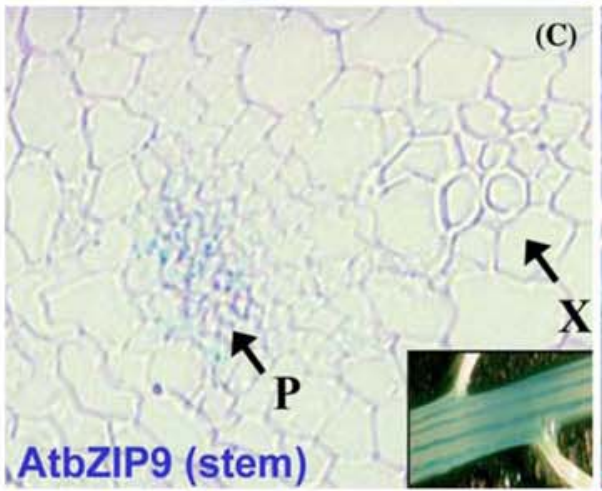

(C)

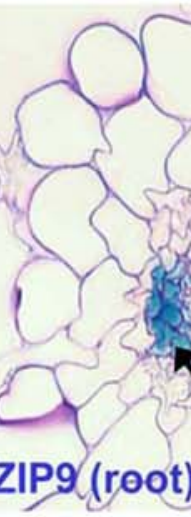

(B) 
(A)

\begin{tabular}{|l|l|l|l|l|l|l|l|l|l|}
\hline \multirow{2}{*}{ AtbZIP: } & \multicolumn{4}{|l}{ Group S1 AtbZIP } & \multicolumn{4}{|c|}{ Group C AtbZIP } \\
\cline { 2 - 12 } & 1 & 2 & 11 & 44 & 53 & 9 & 10 & 25 & 63 \\
\hline young leaves & + & + & + & + & + & $+^{\mathrm{P}}$ & + & - & + \\
\hline old leaves & - & - & - & - & - & - & - & - & - \\
\hline stipulae & + & + & + & + & + & $(+)$ & $(+)$ & + & $(+)$ \\
\hline trichomes & + & + & $(+)$ & $(+)$ & $(+)$ & - & + & - & $(+)$ \\
\hline hydathodes & $(+)$ & $(+)$ & $(+)$ & $(+)$ & $(+)$ & - & $(+)$ & - & - \\
\hline stem & $(+)$ & $(+)$ & $(+)$ & $(+)$ & $(+)$ & $+{ }^{P}$ & $(+)$ & $(+)$ & - \\
\hline root & + & + & + & $(+)$ & $(+)$ & $+{ }^{P}$ & + & $(+)$ & + \\
\hline flower buds & + & + & + & + & + & + & + & + & + \\
\hline carpel & $(+)$ & + & $(+)$ & + & - & + & $(+)$ & $(+)$ & - \\
\hline pollen & $(+)$ & $(+)$ & - & - & $(+)$ & - & - & $(+)$ & $(+)$ \\
\hline connectivum & - & - & + & + & - & + & + & - & - \\
\hline young siliques & $\mathrm{v}$ & f.v & f, s & f.s & s & f & f, s & v & f,v \\
\hline
\end{tabular}

(B)

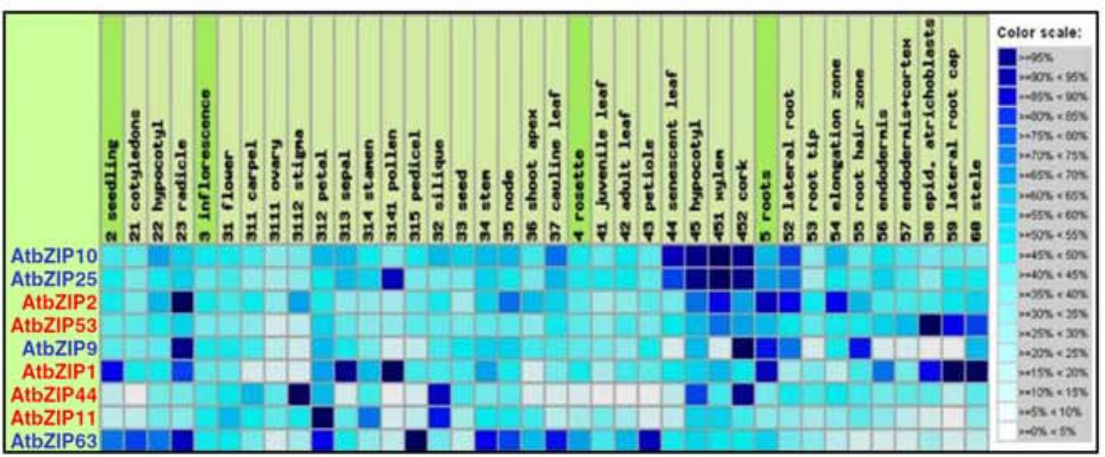

Fig. 7 Comparison of expression data for group S1 (red) and group C (blue) bZIP TFs obtained by histochemical GUS analysis and transcriptome profiling. (a) Summary of GUS expression patterns; +: stable, significant GUS activity: -: no GUS activity; (+): occasional GUS activity appearing in seemingly stochastic manner; ${ }^{\mathrm{P}}$ : GUS activity restricted to the phloem: f: GUS activity mostly in the funiculus; v: GUS activity mostly in the silique valves; s: GUS activity mostly in the developing seeds. Results are based on histochemical analysis of 3-6 independent lines for each bZIP gene, (b) Abundance of AtbZIP transcripts in different plant organs. Given are the modified output results from the meta analyzer of Genevestigator (Zimmermann et al. 2004). This tool illustrates the transcript

Expression of group $\mathrm{C}$ and $\mathrm{S} 1$ genes is fine tuned by metabolic signals on transcriptional and post-transcriptional levels

In agreement with the postulated function of group S1 bZIPs in energy homeostasis, many bZIP genes of the abundance of the indicated genes in different organs, and groups of genes with similar expression patterns by hierarchical clustering. Callus and cell suspension were excluded, as they are not topic of this article. Hierarchical clustering results were generated by pairwise Euclidean distances and using the average linkage method. Results are given as heat maps in blue/white coding that reflect absolute signal values, where a darker colour represents stronger expression. For the blue/white scale, all gene-level profiles were normalized for coloring such that for each gene the highest signal intensity obtains the value $100 \%$ (dark blue) and absence of a signal obtains the value $0 \%$ (white)

$\mathrm{C} / \mathrm{S} 1$ network are regulated by sugars on the transcriptional and post-transcriptional level. In comparison, various hormone treatments barely affect transcription of the analyzed bZIP genes (Zimmermann et al. 2004). Micro array experiments were recently performed with seedlings treated for $1 \mathrm{~h}$ with glucose or with mannitol, 
which served as a control for osmotic stress ( $\mathrm{Li}$ et al. 2006). Strong repression of transcription was observed for AtbZIP1, AtbZIP63 and AtbZIP9, whereas AtbZIP11 was induced (Supplementary Fig. 2). The importance of AtbZIP1 and AtbZIP63 in energy deprivation is further demonstrated by the fact that the highest signals detected for these genes in all 2,507 Genevestigator data sets (Zimmermann et al. 2004) were observed in plants grown for 4 days in the dark without supplementary sucrose and in starving cell cultures. These results were supported by array analysis performed by Price et al. (2004). Darkgrown, sugar-depleted plants were transferred for three hours to hydroponic media supplemented with $3 \%$ glucose or 3\% 3-o-methylglucose (3-OMG) which is not sensed by plants. Glucose treatment strongly repressed expression of AtbZIP1 ( $>30$-fold) and AtbZIP63 (20-fold) and their transcripts were hardly detectable after treatment. Repression of AtbZIP9 and AtbZIP2 was less pronounced. In contrast, the same treatment caused a strong induction of AtbZIP11. 3-OMG did not affect the expression of these genes.

In addition to transcriptional control, the post-transcriptional SIRT mechanism was shown to be effective for AtbZIP11 (Wiese et al. 2004; Hanson et al. 2008). To visualize the effect of the SIRT mechanism in transgenic Arabidopsis seedlings, approximately $500 \mathrm{bp}$ of the group S1 bZIP $5^{\prime}$ leaders sequences were fused to the GUS gene and the expression was driven by the constitutive $U B Q 10$ promoter (Fig. 8 a). In comparison to the mock- or sorbitol-treated controls, growth on $100 \mathrm{mM}$ sucrose resulted in strongly reduced GUS activity mainly in the aerial parts of the seedlings (Fig. 8). Differences in mRNA levels were not responsible for the GUS activity levels, as determined by real time quantitative RT-PCR analysis (data not shown). Although these data clearly demonstrate that the uORF regulation is conserved among all group S1 bZIPs, transcriptional regulation differs. For instance, AtbZIP1 transcription is repressed by glucose, whereas AtbZIP11 is strongly induced (Supplementary Fig. 2). Strong conservation of uORF leader sequences of S1 bZIPs indicates that the SIRT mechanism is likely conserved over the plant kingdom (Wiese et al. 2004). Such posttranslational regulation offers the advantage of added regulatory potential, or, of more rapid responsiveness and fine tuning compared to transcriptional control. It is tempting to speculate that SIRT may serve as an important regulatory circuit to fine tune the S1 expression with respect to the nutrient status of the cell. This hypothesis could also explain variability in GUS staining, as SIRT offers the opportunity to sense local differences in sugar concentrations even at the cellular level and to adapt expression accordingly.
(A)
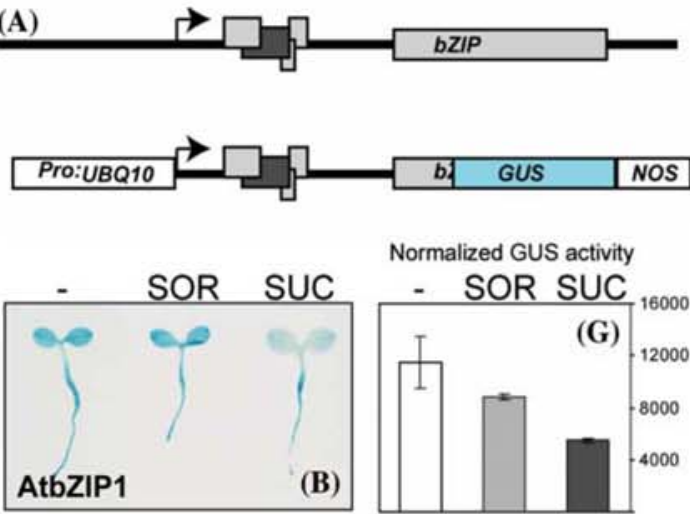

Normalized GUS activity
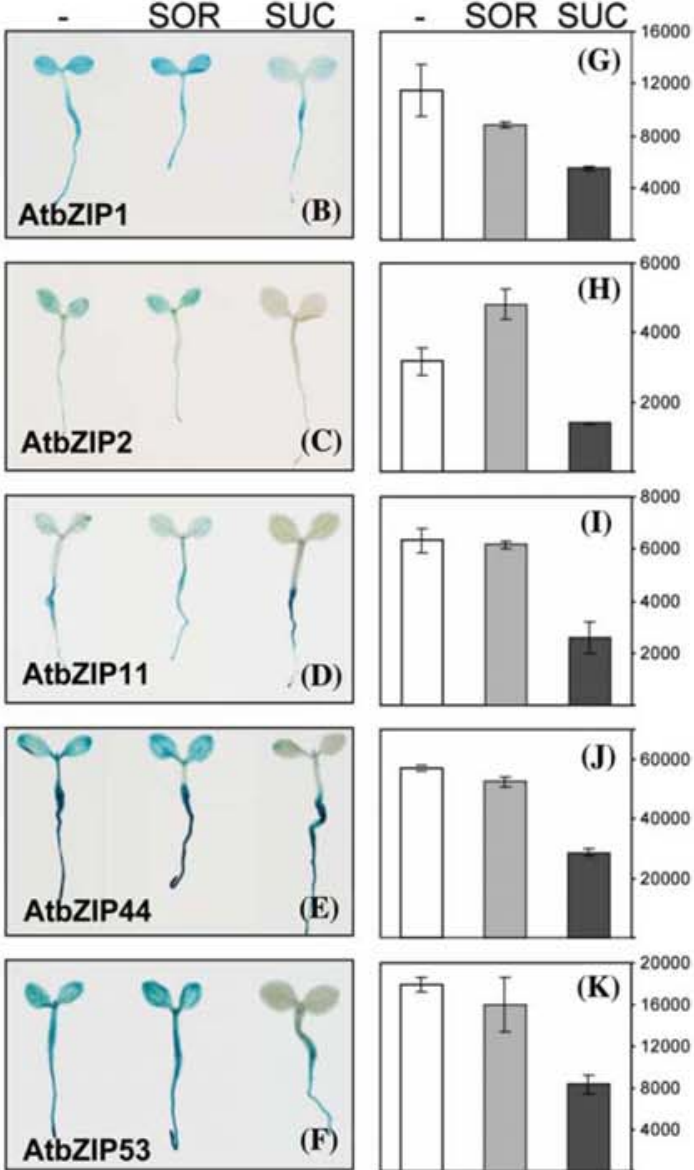

Fig. 8 The leaders of all group S1 AtbZIP genes repress translation in response to sucrose (SIRT, sucrose induced repression of translation), (a) Schematic illustration of uORF arrangement in leaders of five close members of S1 class bZIPs in Arabidopsis thaliana and the T-DNA used for transformation. Arrows represent transcriptional start sites. The uORFs are depicted as gray boxes in three different reading frames. The conserved reading frame is depicted in dark gray. Wt genomic arrangement (top) and T-DNA structure (below). In which the endogenous promoter is replaced by the UBQIO promoter (Pro ${ }$ BO 10 ) and the GUS gene is inserted into the coding sequence of the gene. (b)-(f) Histochemical GUS staining of 5-day-old transgenic Arabidopsis seedlings grown in media without sugar added (left), with $100 \mathrm{mM}$ sorbitol (SOR) (middle) or $100 \mathrm{mM}$ sucrose (SUC) (right). Seedlings were grown for 4 days without sugar added to the media before treatment ( $24 \mathrm{~h}$ duration). (g)-(k) Normalized GUS activity levels of the seedlings shown in (b)-(f). GUS activities were measured using fluorometric MUG assay and normalized to the concentration of soluble proteins in the extracts 
Group S1 AtbZIPs show distinct and significant changes in expression in response to abiotic stresses

The transcription of group S1 AtbZIP genes is affected by different abiotic stress stimuli. The complexity of the S1 AtbZIP transcriptional regulation is demonstrated by the AtGenExpress transcriptome data set (Kilian et al. 2007), in which stressed roots and leaves were analyzed separately (Supplementary Fig. 4). Application of different abiotic stresses often led to an induction of S1 AtbZIP genes in one part of the plant, whereas no effect or an inverse effect was observed in the other. For example, cold and salt stress caused a very strong induction of AtbZIPI in roots, whereas its expression was repressed in leaves. In addition, AtbZIP53 was strongly induced by salt stress in roots, while the transcript level remained unchanged in leaves. This induction was highly specific for salt stress, because the application of osmotic stress had no effect. AtbZIP44 also showed a very strong and specific induction after cold treatment in the root, but no response in the aerial tissues. AtbZIP11 was stress-induced in leaves but not in roots; most strongly by cold stress but also by osmotic and salt stress. Several stress responses have been shown to be mediated by plant hormones (Fujita et al. 2006). However, only transcriptions of AtbZIP1 and AtbZIP9 have been shown to be slightly induced in response to abscisic acid (ABA) (Zimmermann et al. 2004).

With the exception of AtbZIP63, group C genes were hardly regulated at the transcriptional level by the different stress stimuli. However, LSD1-regulated nuclear translocation of AtbZIP10 (see below) might serve as a paradigm for post-translational regulatory mechanism of group C TFs in stress responses (Kaminaka et al. 2006).

In contrast to abiotic stress, group $\mathrm{C}$ and $\mathrm{S} 1$ members show only moderate transcriptional responses to biotic stresses. AtbZIP10 has been described to be involved in the defense responses to the biotrophic pathogen Hyaloperonospora parasitica (Kaminaka et al. 2006). Nevertheless, this regulation occurs on post-translational level via the intracellular distribution of AtbZIP10 by the cell-death regulator LSD1. Group S1-related bZIP factors in other species have been implicated in biotic stress response (Lee et al. 2002). However, only minor transcriptional changes have been observed in the Arabidopsis system. After infection with Pseudomonas syringae, AtbZIP1 showed a 5-fold induction with very slow kinetics (Supplementary Fig. 3). In contrast, AtbZIP11 was induced $3-4$-fold $6 \mathrm{~h}$ post-infection.

\section{Concluding remarks}

Here we examine the $\mathrm{C} / \mathrm{S} 1$ network consisting of nine bZIP transcription factors by focusing on developmental and stress related expression patterns. A characteristic property of the $\mathrm{C} / \mathrm{S} 1$ network is an enhanced gene activation potential brought about by bZIP heterodimers (Ehlert et al. 2006; Weltmeier et al. 2006). Hence, co-expression of at least two bZIP proteins is required to obtain significant target gene transcription. Thus, depending on protein availability in a given cellular context, the C/S1 network of bZIP factors provides an efficient mechanism to integrate signals. With respect to the $2 S 2$ target gene, which is involved in synthesis of storage compounds during seed maturation, we could show that although several C/S1 heterodimers share the properties to activate this target gene, expression pattern and, consequently, protein abundance limits the in planta function of the bZIPs. Analysis of loss-of-function lines is needed to clarify functional redundancies among the bZIP proteins.

As a basis for further functional studies we performed a detailed expression analysis of the C/S1 bZIP genes. In general, all the members of this network show sink specific expression in young leaves, anthers and in seeds, which supports the idea that the C/S1 network is involved in allocation of nutrients to sink tissues (Rook et al. 1998a, 1998b). Constitutive expression of $A S N I$, under the control of the $\mathrm{Pro}_{355}$, results in an enhanced nitrogen status of the seeds (Lam et al. 2003). Accordingly, using independent array approaches, $A S N 1$ has been confirmed as a potential target gene of members of the C/S1 bZIP network (Hanson et al. 2008; FW and WD-L, unpublished results). Further studies have to prove the hypothesis that the $\mathrm{C} / \mathrm{S} 1$ network co-ordinates sink specific genes regulation, basic amino acid metabolism as well as the control of seed storage compounds.

Our expression data indicate that the protein availability of C/S I bZIP TFs is mediated by tissue- and developmental factors as well as differences in the metabolic status which is controlled partly by the post-translational SIRT mechanism. In addition to metabolic signals, abiotic and biotic stress stimuli also result in changes in the transcriptional patterns of C/S1 bZIP genes, which affect the cellular amount of the bZIP proteins. However, stress and metabolic signals mainly influence the expression of group S1 bZIPs and do not affect the expression of group C bZIPs. Consequently, the intrinsic properties of the complex $\mathrm{C} / \mathrm{S} 1$ heterodimerization network might facilitate integration of metabolic and environmental signals and allow rapid integrated responses to the biotic and abiotic environment.

The SnRK1 pathway has been proposed to adjust growth and development in response to the plant's energy status (Baena-Gonzalez et al. 2007). The transcriptional potential of S1 class bZIP proteins is augmented by the SnRK1 pathway. However, the regulatory circuits of the bZIP network seem to be complex and our current knowledge is limited. System biology tools are required to correlate 
extensive data sets on expression, heterodimerization, transactivation properties, post-transcriptional and posttranslational regulation that result in the activation of target genes. These studies will provide further insight into how the energy status of the cell is signaling growth and development.

Acknowledgments We are grateful to C. Carsjens (University of Göttingen, Germany) and F. de Courcy (University of Tübingen. Germany) for proofreading and to K. Demchenko (Komarov Botanical Institute. Russia) for assistance during preparation of sections. This research was supported, in part, by grants from the DFG to WDL and $\mathrm{KH}$ and the European Community to WDL.

Open Access This article is distributed under the terms of the Creative Commons Attribution Noncommercial License which permits any noncommercial use, distribution, and reproduction in any medium, provided the original author(s) and source are credited.

\section{References}

Baena-Gonzalez E, Rolland F, Thevelein JM, Sheen J (2007) A central integrator of transcription networks in plant stress and energy signalling. Nature 448:938-942. doi:10.1038/nature06069

Bensmihen S, To A, Lambert G, Kroj T, Giraudat J, Parcy F (2004) Analysis of an activated ABI5 allele using a new selection method for transgenic Arabidopsis seeds. FEBS Lett 561:127131. doi: 10.1016/S0014-5793(04)00148-6

Boyes DC, Zayed AM, Ascenzi R, McCaskill AJ. Hoffman NE, Davis KR, Gorlach J (2001) Growth stage-based phenotypic analysis of Arabidopsis: a model for high throughput functional genomics in plants. Plant Cell 13:1499-1510

Ehlert A, Weltmeier F, Wang X, Mayer CS, Smeekens S, VicenteCarbajosa J, Dröge-Laser W (2006) Two-hybrid protein-protein interaction analysis in Arabidopsis protoplasts: establishment of a heterodimerisation map of group $\mathrm{C}$ and $\mathrm{S}$ bZIP transcription factors. Plant J 46:890-900. doi:10.1111/j.1365-313X.2006. 02731.x

Fujita M, Fujita Y, Noutoshi Y, Takahashi F, Narusaka Y, Yamaguchi-Shinozaki K, Shinozaki K (2006) Crosstalk between abiotic and biotic stress responses: a current view from the points of convergence in the stress signaling networks. Curr Opin Plant Biol 9:436-442. doi:10.1016/j.pbi.2006.05.014

Hanson J, Hanssen M, Wiese A. Hendriks MWB, Smeekens S (2008) The sucrose regulated transcription factor bZIP11 affects amino acid metabolism by regulating the expression of Asparagin synthase 1 and Proline dehydrogenase 2. Plant J 53:935-949. doi: 10.1111/j.1365-313X.2007.03385.x

Ito K, Kusano T, Tsutsumi K-I (1999) A cold-inducible bZIP protein gene in radish root regulated by calcium- and cycloheximidemediated signals. Plant Sci 142:57-65. doi:10.1016/S0168-9452 (98)00250-7

Jakoby M, Weisshaar B, Dröge-Laser W, Vicente-Carbajosa J. Tiedemann J, Kroj T, Parcy F (2002) bZIP transcription factors in Arabidopsis. Trends Plant Sci 7:106-111. doi:10.1016/ S1360-1385(01)02223-3

Kaminaka H, Nake C, Epple P, Dittgen J, Schütze K. Chaban C. Holt BF, Merkle T, Schäfer E, Harter K. Dangl J (2006) bZIP10LSD1 antagonism modulates basal defense and cell death in Arabidopsis following infection. EMBO J 25:4400-4411. doi: 10.1038/sj.emboj.7601312
Kilian J, Whitehead D, Horak J, Wanke D, Weinl S, Batistic O, D'Angelo C, Bornberg-Bauer E, Kudla J, Harter K (2007) The AtGenExpress global stress expression data set: protocols. evaluation and model data analysis of UV-B light, drought and cold stress responses. Plant J 50:347-363. doi:10.1111/j.1365313X.2007.03052.x

Kusano T, Berberich T, Harada M, Suzuki N, Sugawara K (1995) A maize DNA-binding factor with a bZIP motif is induced by low temperature. Mol Gen Genet 248:507-517. doi:10.1007/ BF02423445

Lam HM, Hsieh MH, Coruzzi G (1998) Reciprocal regulation of distinct asparagine synthetase genes by light and metabolites in Arabidopsis thaliana. Plant J 16:345-353, doi:10.1046/j.1365 313x.1998.00302.x

Lam HM, Peng SS, Coruzzi GM (1994) Metabolic regulation of the gene encoding glutamine-dependent asparagine synthetase in Arabidopsis thaliana. Plant Physiol 106:1347-1357. doi: 10.1104/pp.106.4.1347

Lam HM, Wong P, Chan HK, Yam KM, Chen L, Chow CM, Coruzzi GM (2003) Overexpression of the ASNI gene enhances nitrogen status in seeds of Arabidopsis. Plant Physiol 132:926-935. doi: 10.1104/pp.103.020123

Landschulz WH. Johnson PF. McKnight SL (1988) The leucine zipper: a hypothetical structure common to a new class of DNA binding proteins. Science 240:1759-1764. doi:10.1126/science. 3289117

Lara P. Onate-Sanchez L, Abraham Z, Ferrandiz C. Diaz I. Carbonero P. Vicente-Carbajosa J (2003) Synergistic activation of seed storage protein gene expression in Arabidopsis by $\mathrm{ABI} 3$ and two bZIPs related to OPAQUE2. J Biol Chem 278:21003-21011. doi: $10.1074 /$ jbc.M210538200

Lee SJ, Lee MY, Yi SY, Oh SK, Choi SH, Her NH, Choi D, Min BW, Yang SG, Harn CH (2002) PPI1: a novel pathogen-induced basic region-leucine zipper (bZIP) transcription factor from pepper Mol Plant Microbe Interact 15:540-548. doi:10.1094/MPMI. 2002.15.6.540

Li Y. Lee KK. Walsh S. Smith C. Hadingham S, Sorefan K. Cawley G, Bevan MW (2006) Establishing glucose- and ABA-regulated transcription networks in Arabidopsis by microarray analysis and promoter classification using a Relevance Vector Machine. Genome Res 16:414 427. doi:10.1101/gr.4237406

Polge C. Thomas M (2007) SNF1/AMPK/SnRK1 kinases, global regulators at the heart of energy control. Trends Plant Sci 12:2028. doi:10.1016/j.tplants.2006.11.005

Price J, Laxmi A, St Martin SK, Jang JC (2004) Global transcription profiling reveals multiple sugar signal transduction mechanisms in Arabidopsis. Plant Cell 16:2128-2150. doi:10.1105/tpe. 104.022616

Rook F. Gerrits N. Kortstee A, van Kampen M. Borrias M, Weisbeek P, Smeekens S (1998a) Sucrose-specific signalling represses translation of the Arabidopsis ATB2 bZIP transcription factor gene. Plant J 15:253-263. doi: 10.1046/j.1365-313X.1998.00205.x

Rook F, Weisbeek P. Smeekens S (1998b) The light-regulated Arabidopsis bZIP transcription factor gene ATB2 encodes a protein with an unusually long leucine zipper domain. Plant Mol Biol 37:171-178. doi:10.1023/A:1005964327725

Sambrook J, Fritsch EF, Maniatis T (1989) Molecular cloning: a laboratory manual. Cold Spring Harbour Laboratory Press. New York

Satoh R, Nakashima K, Seki M, Shinozaki K, Yamaguchi-Shinozaki K (2002) ACTCAT, a novel cis-acting element for proline- and hypoosmolarity-responsive expression of the ProDH gene encoding proline dehydrogenase in Arabidopsis. Plant Physiol 130:709-719. doi: 10.1104/pp.009993

Schmid M. Davison TS, Henz SR, Pape UJ, Demar M, Vingron M, Scholkopf B, Weigel D, Lohmann JU (2005) A gene expression 
map of Arabidopsis thaliana development. Nat Genet 37:501506. doi: $10.1038 / \mathrm{ng} 1543$

Shimizu H, Sato K, Berberich T, Miyazaki A, Ozaki R, Imai R. Kusano T (2005) Lip19, a basic region leucine zipper protein, is a fos-like molecular switch in the cold signaling of rice plants. Plant Cell Physiol 46:1623-1634. doi:10.1093/pcp/pci178

Stankovic B, Vian A. Henry-Vian C, Davies E (2000) Molecular cloning and characterization of a tomato cDNA encoding a systemically wound-inducible bZIP DNA-binding protein. Planta 212:60-66. doi: 10.1007/s004250000362

Weigel R, Glazebrook J (2002) Arabidopsis: a laboratory manual. Cold Spring Harbour Laboratory Press, New York

Weltmeier F, Ehlert A, Mayer CS, Dietrich K, Wang X, Schütze K. Harter K, Vicente-Carbajosa J, Dröge-Laser W (2006)
Combinatorial control of Arabidopsis proline dehydrogenase transcription by specific heterodimerisation bZIP transcription factors. EMBO J 25:3133-3143, doi:10.1038/sj.emboj.7601206

Wiese A. Elzinga N. Wobbes B. Smeekens S (2004) A conserved upstream open reading frame mediates sucrose-induced repression of translation. Plant Cell 16:1717-1729. doi:10.1105/tpc. 019349

Zakharov A, Giersberg M, Hosein F, Melzer M, Muntz K, Saalbach I (2004) Seed-specific promoters direct gene expression in nonseed tissue. J Exp Bot 55:1463-1471. doi:10.1093/jxb/erh158

Zimmermann P, Hirsch-Hoffmann M, Hennig L, Gruissem W (2004) GENEVESTIGATOR. Arabidopsis microarray database and analysis toolbox. Plant Physiol 136:2621-2632. doi:10.1104/pp. 104.046367 


\section{5 bZIP heterodimers induce transcriptional activity of target genes in hypoosmotic response}

\section{Combinatorial control of Arabidopsis proline dehydrogenase transcription by specific heterodimerisation of bZIP transcription factors}

\author{
Fridtjof Weltmeier ${ }^{1,4}$, Andrea Ehlert ${ }^{1,4}$, \\ Caroline S Mayer ${ }^{1}$, Katrin Dietrich ${ }^{1}$, Xuan \\ Wang $^{1}$, Katia Schütze ${ }^{2}$, Rosario Alonso ${ }^{3}$, \\ Klaus Harter ${ }^{2}$, Jesús Vicente-Carbajosa ${ }^{3}$ \\ and Wolfgang Dröge-Laser ${ }^{1, *}$
}

${ }^{1}$ Albrecht-von-Haller Institut, Universität Göttingen, Göttingen, Germany, ${ }^{2}$ Zentrum für Molekularbiologie der Pflanzen,

Pflanzenphysiologie, Tübingen, Germany and ${ }^{3}$ Department of Bioquimica y Biologia Molecular, ETSI Agronomos, Universidad Politecnica, Ciudad Universitaria, Madrid, Spain

Proline metabolism has been implicated in plant responses to abiotic stresses. The Arabidopsis thaliana proline dehydrogenase (ProDH) is catalysing the first step in proline degradation. Transcriptional activation of ProDH by hypo-osmolarity is mediated by an ACTCAT cis element, a typical binding site of basic leucine zipper (bZIP) transcription factors. In this study, we demonstrate by gain-of-function and loss-of-function approaches, as well as chromatin immunoprecipitation (ChIP), that ProDH is a direct target gene of the group-S bZIP factor AtbZIP53. Dimerisation studies making use of yeast and Arabidopsis protoplast-based two-hybrid systems, as well as bimolecular fluorescence complementation (BiFC) reveal that AtbZIP53 does not preferentially form dimers with group-S bZIPs but strongly interacts with members of group-C. In particular, a synergistic interplay of AtbZIP53 and group-C AtbZIP10 was demonstrated by colocalisation studies, strong enhancement of ACTCAT-mediated transcription as well as complementation studies in atbzip53 atbzip10 T-DNA insertion lines. Heterodimer mediated activation of transcription has been found to operate independent of the DNA-binding properties and is described as a crucial mechanism to modulate transcription factor activity and function.

The EMBO Journal (2006) 25, 3133-3143. doi:10.1038/ sj.emboj.7601206; Published online 29 June 2006 Subject Categories: chromatin \& transcription; plant biology Keywords: Arabidopsis thaliana; bZIP transcription factors; heterodimerisation; hypo-osmolarity response; $\mathrm{ProDH}$ transcription
"Corresponding author. Albrecht-von-Haller Institut, University of Götiingen, Untere Karspüle 2, Göttingen 37073, Germany. Tel.: + 49 (0)551 39 19816; Fax: + 49 (0)551 397820 ;

E-mail: wdroege@gwdgde

${ }^{4}$ These authors contributed equally to this work

Received: 19 December 2005; accepted: 30 May 2006; published online: 29 June 2006

\section{Introduction}

Osmotic stress, caused by drought, high salinity and cold is a key determinant of plant growth and productivity (Hoekstra et al, 2001). In response to environmental conditions causing osmotic stress, many higher plants accumulate protective compounds, like the compatible osmolyte L-proline (L-Pro) (Delauney et al, 1993; Liu and Zhu, 1997; Parvanova et al, 2004). This compound has been proposed to participate in the protection of plasma membrane integrity (Mansour, 1998) and to act as a scavenger of radicals (Smirnoff and Cumbes, 1989). In addition, L-Pro is discussed to be a source of reducing power (Walton and Boldingh, 1991) and to serve as a transient storage of carbon and nitrogen (Peng et al, 1996).

The L-Pro homeostasis is tightly regulated by its synthesis (Delauney et al, 1993), degradation (Verbrüggen et al, 1996) and transport (Rentsch et al, 1996). L-Pro is synthesised in the cytosol from glutamine or ornithin precursors (Figure 1A). In osmotically stressed tissues, L-Pro mainly results from the glutamine pathway by the activity of the NADPH using enzymes $\Delta^{1}$-pyrroline-5-carboxylate-synthetase (P5CS) and P5C reductase (P5CR) (Delauney et al, 1993; Roosens et al, 1999). L-Pro accumulation correlates with osmotic stress tolerance, however, a stringent causal relationship is not yet firmly established (Nanjo et al, 1999). Furthermore, accumulation of L-Pro is toxic and thus L-Pro and its metabolites have been discussed to function as signalling molecules in stress-induced cell death (Hellmann et al, 2000; Nanjo et al, 2003; Deuschle et al, 2004).

Induced by rehydration, L-Pro is degraded by a pathway localised in the mitochondria, which makes use of the enzymes proline dehydrogenase (ProDH) and P5C dehydrogenase (P5CDH) (Peng et al, 1996; Verbrüggen et al, 1996; Nakashima et al, 1998; Yoshiba et al, 1999). ProDH catalyses the rate-limiting step in-Pro degradation. Transgenic Arabidopsis plants expressing a ProDH antisense construct display high L-Pro levels and enhanced tolerance to freezing conditions and high salinity (Nanjo et al, 1999).

In Arabidopsis thaliana, the ProDH gene (At3g30775) is induced in response to hypo-osmotic conditions occurring during rehydration after stress recovery (Kiyosue et al, 1996). ProDH transcription is also enhanced by high levels of L-Pro and reduced by dehydration (Peng et al, 1996; Verbrüggen et al, 1996). Detailed dissection of the 1.4-kb ProDH promoter revealed an ACTCAT motif which is sufficient for induction of the ProDH gene by L-Pro or hypo-osmolarity, respectively (Nakashima et al, 1998; Satoh et al, 2002). Microarray analysis confirmed that among 121 rehydration-inducible genes, $48 \%$ harbour the ACTCAT motif in their promoters (Oono et al, 2003). These findings indicate that this motif is of general relevance with respect to gene regulation during recovery after osmotic stress. Therefore, ACTCAT-mediated transcription might serve as a fruitful model system for 

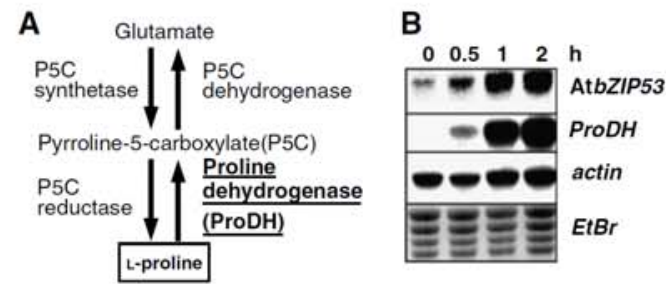

C

atbzip53 (NASC ID: N569883)
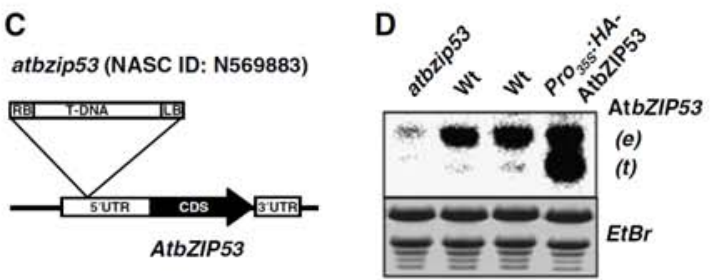

E
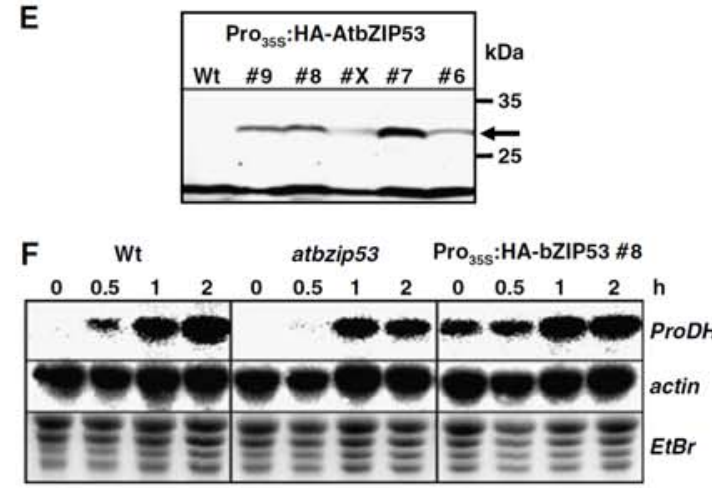

Figure 1 The bZIP transcription factor AtbZIP53 regulates ProDH transcription in the hypo-osmolarity response. (A) Schematic overview of the metabolic pathways involved in L-proline (L-Pro) synthesis and degradation in plant cells. P5CS: $\Delta^{1}$-pyrroline-5carboxylate-synthetase; P5CR: reductase; ProDH: L-Pro dehydrogenase; P5CDH: P5C dehydrogenase (Peng et al, 1996; Verbrüggen et al, 1996; Nakashima et al, 1998; Yoshiba et al, 1999). (B) Gene induction by hypo-osmotic stress treatment. Arabidopsis plants, 14-day-old, were transferred from MS medium ( $58 \mathrm{mM}$ sucrose) to desalted water (Satoh et al, 2004). RNA of control ( $0 \mathrm{~h})$ and stresstreated plants (time points as indicated) was analysed by Northern hybridisation. Depicted are hybridisation experiments with $\mathrm{ProDH}$ and AtbZIP53-specific probes, respectively. (C) Schematic illustration of the T-DNA insertion mutant atbzip53. The insertion was mapped inside the $5^{\prime}$ utr 430 bp upstream of the ATG. (D) Northern analysis of wild-type (Wt), AtZIP53 T-DNA insertion lines (atbzip53), and AtbZIP53-overexpressing plants (Pro ${ }_{355}$ :HA-AtbZIP53). Endogenous (e) and transgene encoded (t) AtbZIPS3 RNAs show slightly different mobility due to the length of their $5^{\prime}$ leaders. (E) Western Analysis of wild-type (Wt) and several independent transformants of $\mathrm{PrO}_{35}$ :HA-AtbZIP53. The transgene-encoded proteins are detected by a HA-specific antibody (arrow). (F) Induction of $\mathrm{ProDH}$ transcription in response to hypo-osmolarity treatment. RNA was isolated after the time points indicated and ProDH transcript level of $\mathrm{Wt}$, atbzip53, and $\mathrm{Pro}_{355}$ :HA-AtbZIP53 plants are compared. Equal loading was verified by ethidium bromide $(\mathrm{EtBr})$ staining and hybridisation with an actin-specific probe. All experiments have been replicated at least three times with similar results.

studying transcriptional control of osmotic stress responses, which is of importance for both, basic science as well as genetic engineering of crop plants.

The ACTCAT-motif is related to the GCN4-binding site (ATGA(C/G)TCAT) (Ellenberger et al, 1992) recognised by members of the basic leucine zipper (bZIP) transcription factor family (Landschulz et al, 1988). In the model plant Arabidopsis thaliana, 75 bZIP genes have been identified and classified into 10 groups (Jakoby et al, 2002). In vitro DNA-binding studies and transfection experiments performed in transiently transformed protoplasts revealed that distinct members of Arabidopsis group-S bZIP proteins are involved in ACTCAT-mediated transcription (Satoh et al, 2004). Although 17 group-S bZIPs have been identified, only a subgroup consisting of five highly related members (AtbZIP1, AtbZIP2, AtbZIP11/ATB2, AtbZIP44 and AtbZIP53) seem to be involved in ProDH regulation (Satoh et al, 2004) and will be referred to as group-S1 bZIPs.

bZIP proteins are phylogenetically widely distributed transcriptional regulators, characterised by a basic DNA-binding domain (b) (Landschulz et al, 1988). Although DNA binding of bZIP monomers has been described, they normally interact with DNA as dimers (Ellenberger et al, 1992; Metallo and Schepartz, 1997; Cranz et al, 2004). Dimerisation is mediated by the so-called leucine zipper domain, a heptad repeat of leucine or other bulky hydrophobic amino acids creating an amphipathic helix (Landschulz et al, 1988; Baxevanis and Vinson, 1993). The formation of bZIP homo- or heterodimers offers a huge combinatorial flexibility to regulatory transcription systems. By heterodimerisation, DNA-binding specificity and affinity, transactivation properties and ultimately, cell physiology might be altered (Naar et al, 2001). For instance, in the animal system, heterodimers of the bZIP proteins Jun and Fos have been shown to recognise the AP1 motif, which is not targeted by Jun homodimers (Kouzarides and Ziff, 1988). As bZIP proteins do not heterodimerise promiscuously but specifically (Newman and Keating, 2003), an important function for gene regulation is anticipated. In planta, bZIP heterodimerisation has been shown for closely related bZIPs, for example, the GBFs (Schindler et al, 1992). In tobacco (Strathmann et al, 2001) and parsley (Rügner et al, 2001), specific heterodimerisation was found between members of two groups of bZIPs that are related to the Arabidopsis groups $\mathrm{S}$ and $\mathrm{C}$. Interestingly, heterodimerisation between these groups is strongly preferred in comparison to homotypic dimerisation.

In this work, we demonstrate by means of transgenic and ChIP approaches that ProDH is a direct target gene of the group-S1 bZIP transcription factor AtbZIP53. Using yeast twohybrid, BiFC and interaction assays in protoplasts, we show that AtbZIP53 does not function as a homodimer, but preferentially heterodimerises with group-C bZIP transcription factors. AtbZIP53 and the group-C heterodimerisation partner AtbZIP10 were found to be colocalised and to control ACTCATmediated $\mathrm{ProDH}$ transcription in a synergistic manner. We describe bZIP heterodimerisation as an essential mechanism to modulate transactivation properties of transcriptional regulators in planta. Furthermore, we provide in vivo evidence that $\mathrm{ProDH}$ is not regulated by a single bZIP but by a complex heterodimerisation network of group-S1/C bZIP factors.

\section{Results}

AtbZIP53 directly participates in the hypo-osmolarityinduced transcription of the ProDH gene

Using in vitro DNA-binding studies and transfection experiments in Arabidopsis protoplasts, Satoh et al (2004) demon- 
strated that the group-S1 bZIP transcription factors bind to the ACTCAT cis element located in the ProDH promoter. However, expression data of the correspondent $b Z I P$ genes, obtained from the RARGE database (http://rarge.gsc riken.jp) or by Satoh et al (2004), suggest that AtbZIP53 constitutes as the main regulator of hypo-osmolarity-induced ProDH transcription. Whereas AtbZIP53 is strongly induced by hypo-osmolarity treatment (Figure 1B), AtbZIP11 or AtbZIP44 are not. We therefore focussed on studying the function of AtbZIP53 in the regulation of $\mathrm{ProDH}$ transcription. Expression of an HA-tagged AtbZIP53 gene driven by the 35S promoter $\left(\mathrm{Pro}_{35 s}:\right.$ HA-AtbZIP53), as monitored by Northern and Western analyses (Figure $1 \mathrm{D}$ and E), resulted in a constitutive activation of the ProDH gene (Figure 1F). Comparable results were obtained with several transgenic lines expressing an untagged construct indicating that this response is not due to the HA-tag (data not shown). Complementarily, a partial loss-of-function mutant of AtbZIP53 (atbzip53) (Figure 1C and D) displayed a significantly reduced and delayed induction of $\mathrm{ProDH}$ transcription after hypo-osmolarity treatment (approximately by 40\%) (Figure 1F). Owing to redundancy, AtbZIP53 and other group-S1 bZIP transcription factors (Jakoby et al, 2002) might show an overlapping function which results in the residual activation of ProDH expression in the AtbZIP53 plant.

To verify direct binding of AtbZIP53 to the ProDH promoter in vivo, ChIP was performed applying chromatin obtained from HA-AtbZIP53-expressing plants ( Pro $_{355}$ :HA-AtbZIP53) using an HA-tag-specific antibody. A significant enrichment of ProDH promoter fragments was detected by PCR amplification, using $\mathrm{ProDH}$ promoter-specific primers (Figure 2). Control primers, unrelated to the AtbZIP53 target gene did not result in an enrichment following immunoprecipitation (Figure 2). In summary, the in vitro and in vivo evidence support the hypothesis that the ProDH promoter is a direct target of the AtbZIP53 transcription factor.

AtbZIP53 heterodimerises with group-C bZIP transcription factors in yeast and plant cells bZIP factors usually bind DNA as homo- or heterodimers, consequently, dimer formation allows the establishment of

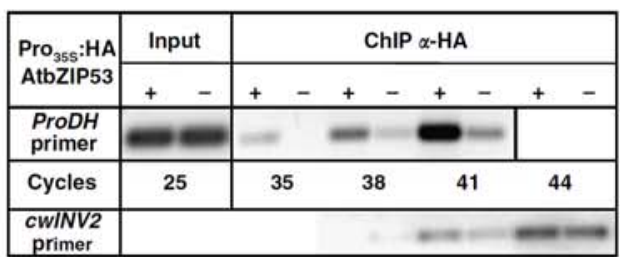

Figure 2 In vivo binding of AtbZIP53 to the ProDH promoter. ChIP analysis of chromatin obtained from Pro ${ }_{35 s}$ :HA-AtbZIP53 $(+)$ and Wt (-) plants using a HA-specific antibody for immunoprecipitation. ProDH primers were used to amplify a $191 \mathrm{bp}$ fragment of the ProDH promoter. Given are the PCR products obtained after 35,38 , or 41 cycles, respectively. As input control, PCR amplicons are shown which have been obtained after 25 cycles with material before immunoprecipitation. The specificity was verified by using primers amplifying the promoter of a nontarget gene (cwINV2, At3g52600). Given are amplicons obtained after 38,41 or 44 cycles, respectively. The PCR products were stained by ethidium bromide. The experiments have been replicated three times with similar results. complex regulatory networks based on combinatorial interactions. It is tempting to speculate that heterodimers of AtbZIP53 formed with the related group-S1 bZIP factors are regulating ProDH transcription. However, when applying a yeast two-hybrid approach, no AtbZIP53 homodimers or AtbZIP53 containing heterodimers were formed with any of the group-S1 bZIP proteins or other less related group-S bZIP factors (Figure $3 \mathrm{~A}$ ). A quantitative assay for protein-protein interactions confirmed the weak heterodimerisation capacity

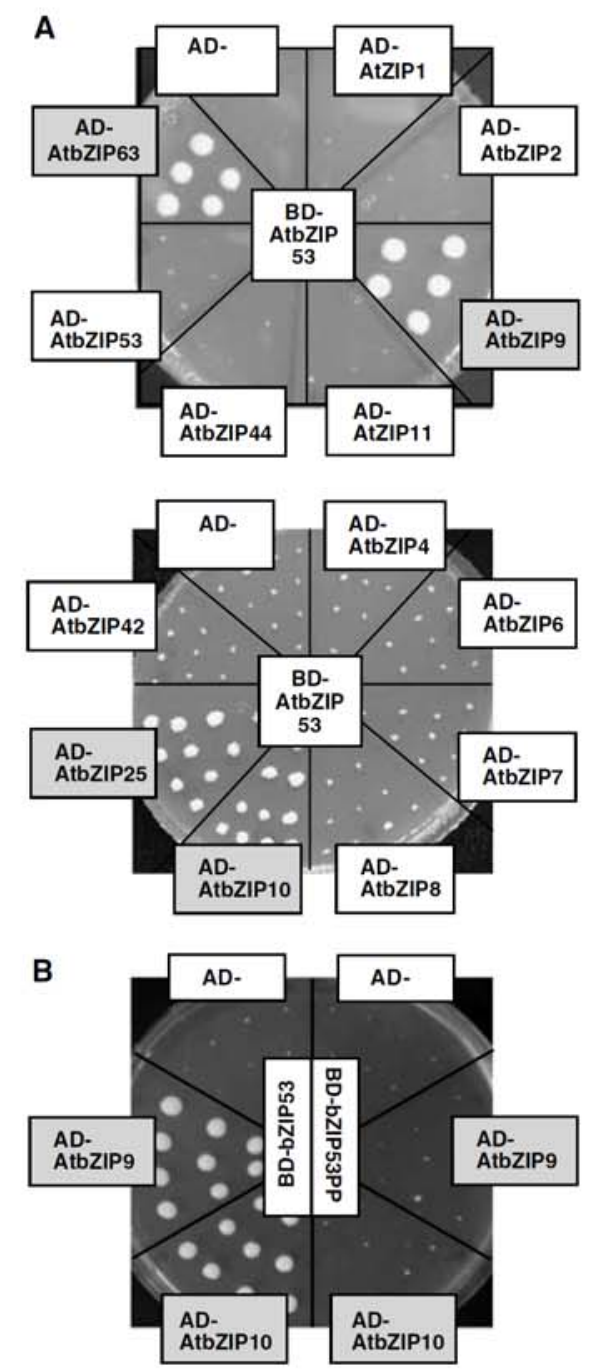

Figure 3 Yeast two-hybrid analysis studying heterodimerisation of AtbZIP53 with group-S and -C bZIP factors. (A) Interaction of BDAtbZIP53 and the AD-bZIP fusion proteins indicated lead to prototrophic growth of the yeast strain MAV203 (ProQuest ", Invitrogen) on medium lacking uracil. Group-C bZIP (AtbZIP63, AtbZIP9, AtbZIP25, AtbZIP10) and group-S bZIP proteins (AtbZIP53, AtbZIP2, AtbZIP44, AtbZIP11, AtbZIP42, AtbZIP7, AtbZIP6, AtbZIP4, AtbZIP8) are labelled by a grey or white background, respectively. (B) Interaction of AtbZIP53 and group-C bZIP proteins is mediated by the ZIP domain. ZIP-specific exchanges of the amino acids leucine ${ }_{51}$ and alanine $e_{72}$ by Pro (AtbZIP53 3 pp) disrupt the $\alpha$-helical structure and results in loss of interaction (right) in comparison to the wild-type AtbZIP53 (left). 
of AtbZIP53 with other group-S1 members (data not shown). We further analysed the potential of AtbZIP53 to interact with other bZIP factors. As described for tobacco (Strathmann et al, 2001) and parsley (Rügner et al, 2001), group-S1-like bZIP proteins specifically heterodimerise with group-C-like bZIPs. Strong heterodimerisation of AtbZIP53 was detected with all Arabidopsis group-C bZIP proteins, namely AtbZIP9, AtbZIP10, AtbZIP25 and AtbZIP63 (Figure 3A). As no heterodimerisation with members of the bZIP groups A (e.g. AtbZIP39) and D (e.g. AtbZIP22) was found, this heterodimerisation is assumed to be specific. In order to analyse whether the protein-protein interaction is mediated by the leucine zipper domain, two conserved residues (leucine ${ }_{51}$ and alanine $_{72}$ ) were changed to prolines which should disrupt the $\alpha$-helical structure of the ZIP domain (AtbZIP53 $3_{\mathrm{PP}}$ ). As shown in Figure 3B, no interaction between AtbZIP53 $3_{\mathrm{pp}}$ and group-C members, such as AtbZIP10 and AtbZIP9 was observed.

To verify bZIP heterodimerisation in planta, BiFC assays were performed in Agrobacterium-infiltrated tobacco (Nicotiana benthamiana) leaves (Walter et al, 2004) using the strongly interacting AtbZIP53 and AtbZIP10 as a representative example. Both bZIP factors localise to the nucleus when expressed as YFP fusion proteins (Figure 4A and B). When fused to the $\mathrm{N}$ - and $\mathrm{C}$-terminal YFP fragment and coexpressed in tobacco leaves, a strong BiFC signal was detected in the nuclear compartment of the transformed cells (Figure 4C), confirming the interaction of AtbZIP53 and AtbZIP10 in planta. Control experiments in which C-YFP-AtbZIP53 and unfused N-YFP protein are expressed do not show any fluorescence (Figure 4D). In order to demon strate the specificity of BiFC system, the C-YFP-AtbZIP53 $3_{\mathrm{Pp}}$ mutation was coexpressed with N-YFP-AtbZIP10. Specific mutation of the ZIP domain (see Figure 3B) interferes with heterodimerisation and in consequence does not result in positive BiFC signals (Figure 4E). In order to confirm expression of the BiFC constructs, Western analyses have been performed (data not shown). Finally, homodimerisation of AtbZIP53 and AtbZIP10 was analysed in this system, but no or only significantly weaker homotypic interactions could be detected (data not shown). In summary, these experiments provide conclusive evidence for the formation of group-S1/C heterodimers in planta.

\section{Heterodimerisation with group-C bZIPs enhances the transactivation properties of AtbZIP53 in planta}

In order to quantify bZIP heterodimerisation in planta, a twohybrid approach was established in Arabidopsis protoplasts as described by Ehlert et al (2006). AtbZIP53 and the related group-S1 and group-C bZIP factors were fused to the GAL4 DNA-binding domain (BD) (Giniger et $a l, 1985$ ) and expressed under control of the $35 \mathrm{~S}$ promoter. Cotransfection with a GUS reporter, which is driven by GAL4 upstream activating sequence (GAL-UAS $\left.{ }_{4}: \mathrm{GUS}\right)$, allows quantification of bZIP activation properties independent of their DNA binding (Figure 5A). Whereas group-S1 members AtbZIP11, AtbZIP44 and AtbZIP2 showed significant activation capacity; AtbZIP53, AtbZIP1 and the group- $\mathrm{C}$ members displayed almost no intrinsic activation (Figure 5B).

In order to study heterodimerisation of AtbZIP53, BDAtbZIP53 was individually coexpressed with GAL4 activation domain (AD) fusions of the bZIP heterodimerisation partners (Figure 5C). Similar to the results obtained in yeast, almost

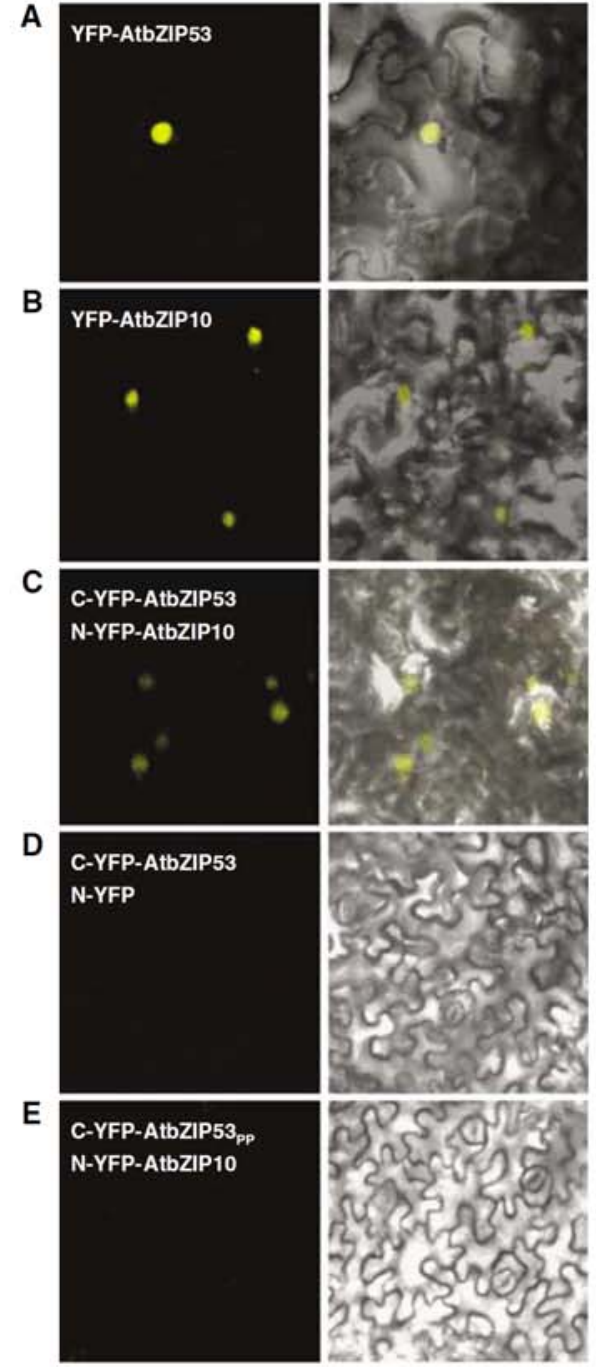

Figure $4 \mathrm{BiFC}$ analysis of AtbZIP53/AtbZIP10 heterodimerisation in planta. Localisation of YFP-AtbZIP53 (A) and YFP-AtbZIP10 (B) fusion proteins in Nicotiana benthamiana leaf epidermal cells after Agrobacterium-mediated transient transformation. (C) Fluorescence observed by confocal microscopy which results from complementation of the C-terminal (C-YFP) and N-terminal (N-YFP) part of the YFP protein fused to AtbZIP53 and AtbZIP10, respectively. (D) Negative control making use of C-YFP-AtbZIP53 and an unfused N-YFP protein. (E) Coexpression of the C-YFP-AtbZIP53pp mutan (see Figure 3B) and N-YFP-AtbZIP10. YFP-Epifluorescence (left) and bright field overlay images (right) are depicted.

no AtbZIP53 homodimerisation and only weak interaction with the group-S1 members AtbZIP2, AtbZIP11 and AtbZIP44 was observed (Figure 5D). A strong heterodimerisation of AtbZIP53 with the group-C members AtbZIP10, AtbZIP25 and AtbZIP9 was detected. In contrast, interaction between AtbZIP53 and group-C AtbZIP63 was less pronounced.

Heterodimerisation might modulate the transactivation capacity of bZIP transcription factors. We therefore coexpressed BD-AtbZIP53 with bZIP partners which have not been fused to the AD domain (Figure 5D). Strong reporter 


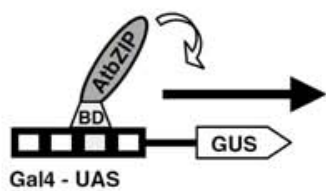

B

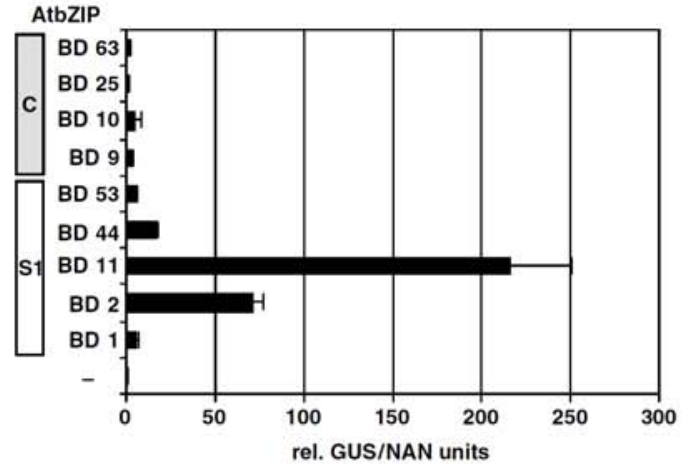

C

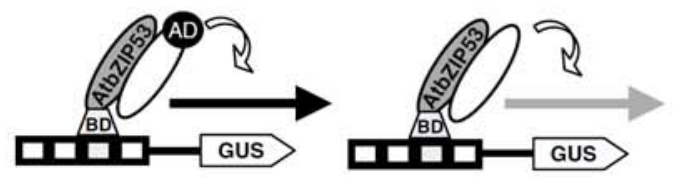

D

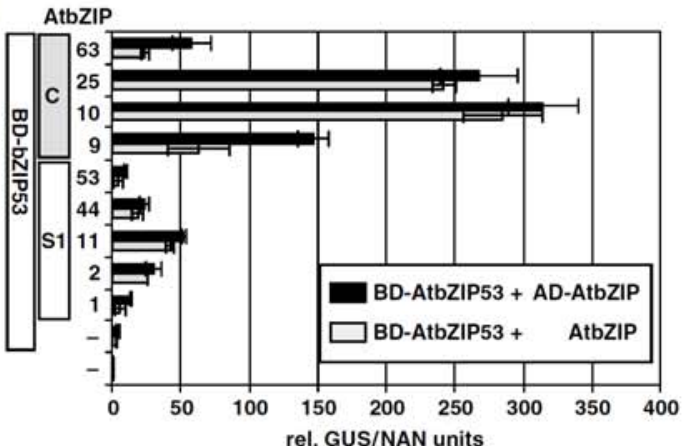

Figure 5 Analysis of AtbZIP53 heterodimerisation in transiently transformed Arabidopsis protoplasts. Experimental set-up (A) to analyse bZIP activation properties. The activation of a GUS reporter driven by multimeric GALA-UAS DNA-binding sites (GALdriven by multimeric GALA-UAS DNA-binding sites (GAL-
UAS $_{4}$ :GUS) was measured after expression of GALA-BD fusions of group-S1 and C bZIP proteins (BD-AtbZIP) (B). Experimental set-up (C) to analyse AtbZIP53 heterodimerisation. Activation of a GAL$\mathrm{UAS}_{4}$ :GUS reporter was measured after coexpression of BDAtbZIP53 and bZIP proteins fused to an AD domain of GAL4 (AD, black bars) or an HA-tag (grey bars) (D). In single transfection experiments, $9 \mu \mathrm{g}$ of reporter and $28 \mu \mathrm{g}$ of effector plasmid DNA were applied. In cotransfections, $14 \mu \mathrm{g}$ of each AtbZIP effector plasmid were used. Variations in transformation efficiency are normalised by using $3 \mu \mathrm{g}$ of a $\mathrm{Pro}_{355}$ :NAN plasmid construct per transfection (Kirby and Kavanagh, 2002). Given are mean values and s.d.'s of four independent transfections as relative GUS/NAN units.

gene activation was obtained for AtbZIP53/AtbZIP10 and AtbZIP53/AtbZIP25 heterodimers. As neither AtbZIP53 nor group-C bZIPs exhibit strong activation on their own (Figure 5B), these data indicate that specific heterodimerisation may act as a key mechanism to modulate transactivation properties in planta. This mechanism will be referred to as heterodimer-induced transactivation (HIT).

In order to analyse the HIT mechanism, the following control has been included into the experimental set-up. Fusing a BD domain to the N-terminus of the bZIP proteins might mask endogenous activation properties of the bZIP proteins analysed in Figure 5B. We therefore coexpressed BD-AtbZIP53 with the other BD-bZIP proteins which also lead to the synergistic reporter gene activation in the case of AtbZIP53/AtbZIP10 and AtbZIP53/AtbZIP25, respectively (data not shown).

The HIT mechanism is not restricted to AtbZIP53/ AtbZIP10 and AtbZIP53/AtbZIP25 heterodimers. A complete analysis of all group- $\mathrm{S} 1 / \mathrm{C}$ interactions, according to the setup described in Figure 5C, revealed that the HIT mechanism is a typical feature of distinct heterodimers (Supplementary Table S1). It is particularly observed between AtbZIP25, AtbZIP10 and most group-S1 members. Moreover, HIT is also observed inside group-C, as demonstrated for AtbZIP10/AtbZIP63. These data suggest that HIT is of general importance for the function of the group-C and S bZIP factors.

Group-S1 and C bZIPs bind specifically the ACTCATregulatory element present in the ProDH promoter

ProDH promoter analysis revealed an ACTCAT motif to be necessary and sufficient for hypo-osmolarity-induced gene activation (Satoh et al, 2002). To assess the significance of AtbZIP53 homo- or heterodimer participation in ACTCATmediated transcriptional control, we transiently expressed AD-bZIP proteins in Arabidopsis protoplasts in conjunction with a reporter vector containing this regulatory element. In this experimental system, activation of a (ACTCAT $)_{2}$ :GUS reporter should solely rely on the binding properties of the bZIP proteins and be independent of their intrinsic activation capacity. The results in Figure 6 show that all bZIP proteins tested are able to interact with this element, and demonstrate that AtbZIP53 binds more effectively the ACTCAT motif than group-C bZIPs. The specificity of this interaction was studied by introducing mutations into the ACTCAT element. The use of a (ACTtta $)_{2}$ :GUS reporter resulted in a loss of activation for all bZIPs, confirming that the ACTCAT motif is a specific binding site for these proteins in planta (Figure 6).

\section{AtbZIP53-containing heterodimers bind the ProDH promoter ACTCAT-cis-element and regulate ACTCAT- mediated transcription}

The results of the heterodimerisation assays prompted us to analyse whether ProDH is regulated by AtbZIP53-containing heterodimers. We therefore coexpressed AtbZIP53 in combination with $\mathrm{AD}$-bZIP fusions of group-S1 and $\mathrm{C}$ bZIPs (Figure 7). As we observed for the GAL-UAS supported transcription, synergistic enhancement of the ACTCATmediated transcription was obtained by coexpression of AtbZIP53 and its group-C heterodimerisation partners AtbZIP9, AtbZIP10, AtbZIP25 and AtbZIP63, respectively. In order to analyse the endogenous activation properties, the bZIP proteins were expressed without the $\mathrm{AD}$ domain (Figure 7). Equal expression of the proteins was monitored by Western blot using an HA-tag-specific antibody (data not shown). Whereas expression of single bZIP proteins resulted in weak reporter activation, a significant synergistic activation was obtained with all AtbZIP53/group-C hetero- 


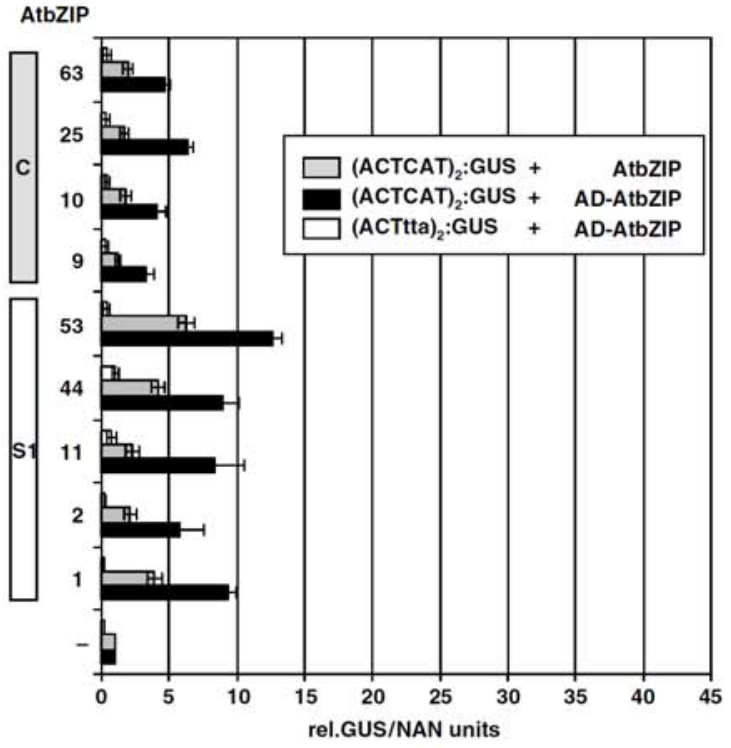

Figure 6 ACTCAT-specific DNA binding of group-S1 and C bZIP factors. Arabidopsis protoplasts were transiently transformed with a GUS reporter construct driven by the ACTCAT motif ((ACTCAT $)_{2}$ :GUS) (black and grey bars) or a mutated version ((ACTttg) :GUS) (white bars). Activation of the reporters was measured after expression of AtbZIP proteins (grey and white bars) or AD-AtbZIP proteins (black bars). In single transfection experiments, $9 \mu \mathrm{g}$ of reporter and $28 \mu \mathrm{g}$ of effector plasmid DNA was applied. Variations in transformation efficiency are normalised by using $3 \mu \mathrm{g}$ of a $\mathrm{PrO}_{35}$ : NAN plasmid construct per transfection (Kirby and Kavanagh, 2002). Given are mean values and s.d.'s of four independent transfections as relative GUS/NAN units.

dimers. Based on these data, a number of heterodimers seem to fulfil a partly redundant function in ACTCAT-mediated transcription.

\section{AtbZIP53 and AtbZIP10 cooperate in regulating hypo- osmolarity induced ProDH transcription}

The data described above suggest that a complex network of heterodimers is involved in regulation of ProDH. To get further insight in this regulatory mechanism, we focused on AtbZIP53 and AtbZIP10, the two partners that show the highest degree of ACTCAT-mediated reporter gene activation. By means of ChIP analysis we could demonstrate that ProDH is a direct target of both, AtbZIP53 (Figure 2) as well as AtbZIP10 (Supplementary Figure S1). Furthermore, electrophoretic mobility shift assays (EMSA) confirmed that heterodimers can be formed on the ACTCAT motif in vitro (Supplementary Figure S2). A detailed experimental series was initiated in which the ratio of bZIP effector plasmids was altered. Figure $8 \mathrm{~A}$ shows that the synergistic effect of AtbZIP53 and AtbZIP10 clearly depends on the ratio of the two heterodimerisation partners. Furthermore, the synergistic effect depends on the ZIP-mediated heterodimerisation function. Disruption of the $\alpha$-helical structure in the AtbZIP53 $3_{\mathrm{PP}}$ construct impairs activation of the reporter by AtbZIP53/ AtbZIP10 (Figure 8B).

To verify the impact of heterodimerisation on reporter gene expression, similar experiments as described above were

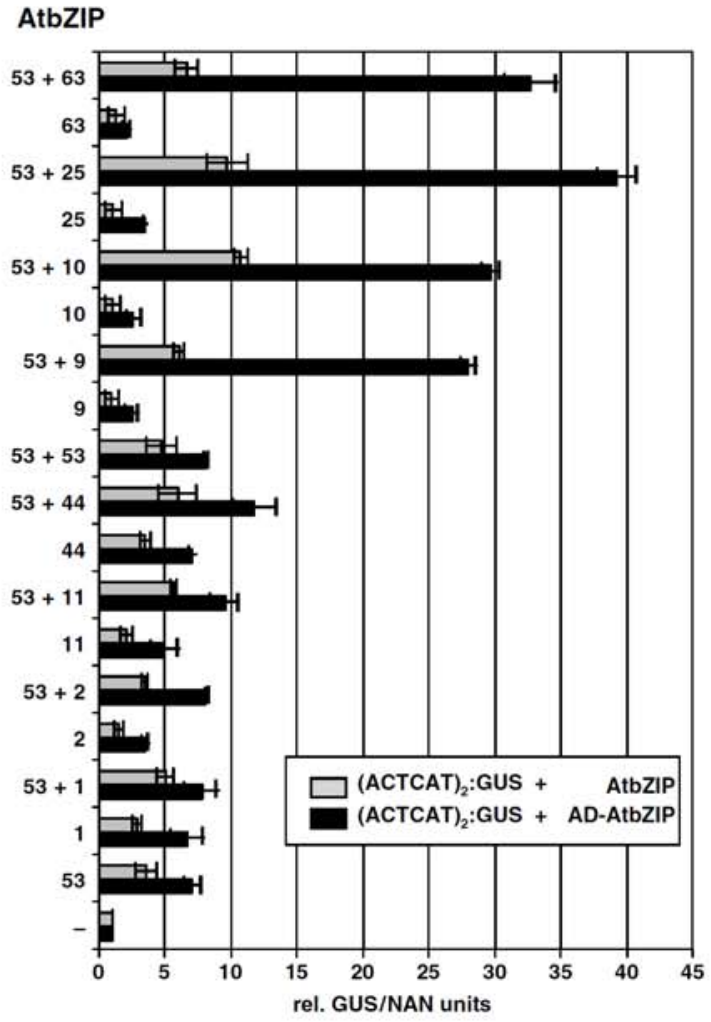

Figure 7 The ACTCAT-mediated transcription is regulated by AtbZIP53 heterodimers. Regulation of the (ACTCAT) $)_{2}$ :GUS reporter by heterodimers was analysed by coexpression of AtbZIP53 and group-S1 and C bZIP (grey bars) or AD-AtbZIP proteins (black bars). In single transfection experiments, $9 \mu \mathrm{g}$ of reporter and $28 \mu \mathrm{g}$ of effector plasmid DNA was applied. In cotransfections, $14 \mu \mathrm{g}$ of each bZIP effector plasmid were used. Variations in transformation efficiency are normalised by using $3 \mu \mathrm{g}$ of a $\mathrm{Pro}_{355}$ :NAN plasmid construct per transfection experiment (Kirby and Kavanagh, 2002). Given are mean values and s.d.'s of four independent transfections as relative GUS/NAN units.

performed in protoplasts obtained from atbzip53 atbzip10 plants. For efficient reporter gene expression, both AtbZIP53 and AtbZIP10 are required (Figure 8D). All together, these data strongly suggest that AtbZIP53/AtbZIP10 heterodimers are regulating ACTCAT-mediated ProDH transcription.

Colocalisation of the bZIP partners in the same tissue is a prerequisite for heterodimerisation. Whereas transcription of AtbZIP53 is strongly induced by hypo-osmolarity (Figure 1B), only slight induction can be obtained for AtbZIP10 (Supplementary Figure S3). Analysis of AtbZIP53 and AtbZIP10 promoter GUS reporter lines ( Pro $_{\mathrm{AtbZIP}_{3}}$ :GUS and

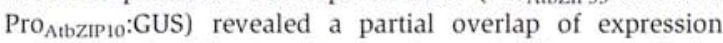
patterns primarily in the meristematic tissues of the Arabidopsis plantlet. Strong enhancement of GUS activity after transfer from MS media to distilled water was found for both, Pro $_{\text {AtbZIP53 }}$ :GUS and Pro AttzIPI0:GUS reporter lines (Figure 9). In contrast, Pro $_{\mathrm{AtbZiP}_{25}}$ :GUS lines do not show this induction, indicating that this bZIP transcription factor is not involved in this response. Coexpression of AtbZIP53 and AtbZIP10 was also observed in parts of the flower and 


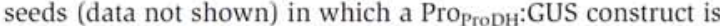
strongly expressed (Nakashima et al, 1998).

In conclusion, these data demonstrate that AtbZIP53 and AtbZIP10 colocalise and interact with high affinity. Moreover, protein availability, which is regulated by environmental and developmental cues, restricts heterodimer formation and ultimately controls target gene transcription (Figure 10).

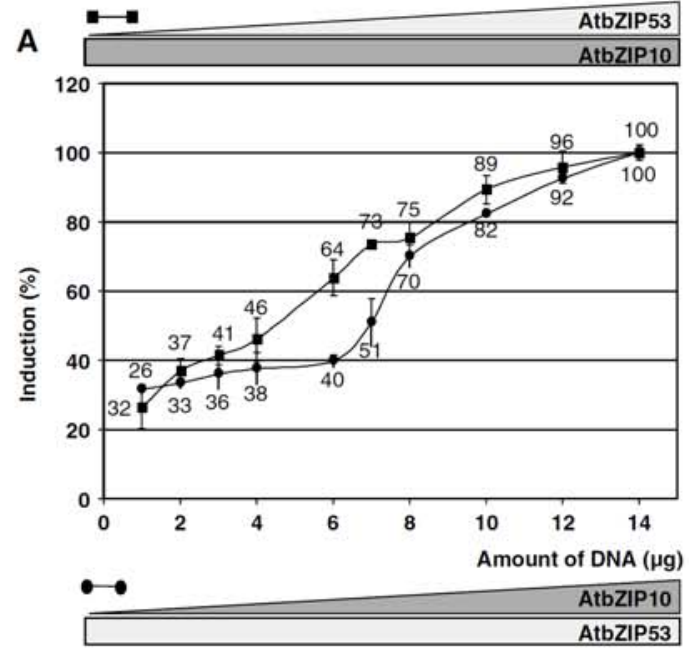

B

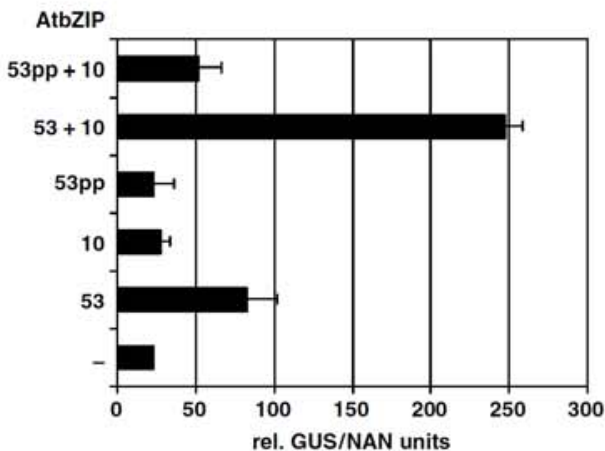

C

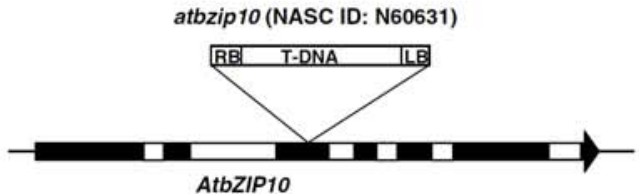

D

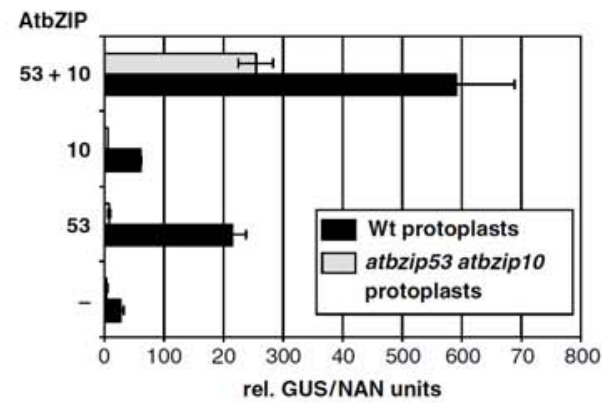

\section{Discussion}

The amino acid L-Pro is essential for basic metabolism as well as for plant development. Moreover, under stress conditions, it has been proposed to serve as a protective compound or as a signalling molecule (Delauney et al, 1993; Liu and Zhu, 1997). Consequently, final effects derived from its availability depend on L-Pro homeostasis, a process tightly regulated by synthesis, degradation and transport. In this work we focus on the transcriptional control of the ProDH gene, encoding the rate-limiting enzyme of L-Pro degradation and found that it is directly regulated by the transcription factor AtbZIP53. We disclosed that AtbZIP53 action is modulated by other bZIP heterodimerising partners that integrate a fine regulatory network supported by combinatorial interactions.

\section{The ProDH promoter ACTCAT motif is a direct target} of AtbZIP53 transcription factors in vivo

Previously reported in vitro DNA-binding studies (Satoh et al, 2004), alongside with the different experimental results presented in this work, including overexpression in transgenic plants, ChIP analysis, and activation of ACTCAT reporter constructs in protoplasts, provide conclusive evidence that

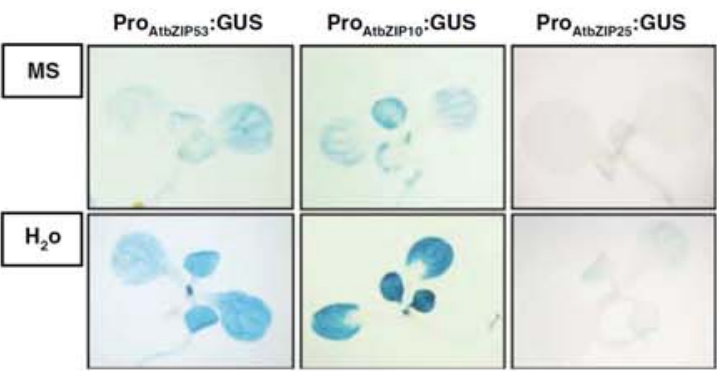

Figure 9 Coexpression studies of AtbZIP53 and AtbZIP10

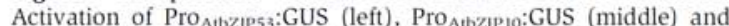

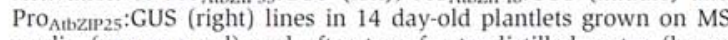
media (upper panel) and after transfer to distilled water (hypoosmolarity treatment) for $12 \mathrm{~h}$ (lower panel).

Figure 8 The ACTCAT-mediated transcription in Arabidopsis protoplasts depends on AtbZIP53/AtbZIP10 heterodimers. (A) Reporter activation depends on the ratio of AtbZIP53 and AtbZIP10. Activation of (ACTCAT) $)_{2}$ GUS reporter was measured using $14 \mu \mathrm{g}$ of AtbZIP10 and increasing amounts of AtbZIP53 encoding plasmid (squares) or $14 \mu \mathrm{g}$ of AtbZIP53 and increasing amounts of AtbZIP10 encoding plasmid (circles), respectively. Given are reporter gene induction values in \%. Reporter gene activation after applying $14 \mu \mathrm{g}$ of each effector is defined as $100 \%$. (B) Activation of the $(\text { ACTCAT })_{2}:$ GUS reporter by AtbZIP53/AtbZIP10 depends on a ZIP-mediated heterodimerisation. ZIP-specific exchanges of the amino acids leucine $e_{51}$ and alanine $_{72}$ by proline (AtbZIP53pp) dis rupts the $\alpha$-helical ZIP structure, bZIP heterodimerisation (see Figure 3B) and reporter gene activation. (C) Graphical description of the T-DNA insertion mutant atbzip10. Exons are depicted as black bars, introns as white bars. The insertion was mapped inside the third exon. (D) Activation of (ACTCAT) $)_{2}$ :GUS reporter by AtbZIP53, AtbZIP10 or both was compared in protoplasts derived from wildtype (black bars) and atbzip53 atbzip10 double mutant plants (grey bars), respectively. For each bZIP encoding construct, $14 \mu \mathrm{g}$ of DNA have been used. Given are relative GUS/NAN units. 
Control of ProDH expression by bZIP heterodimers

F Weltmeier et al

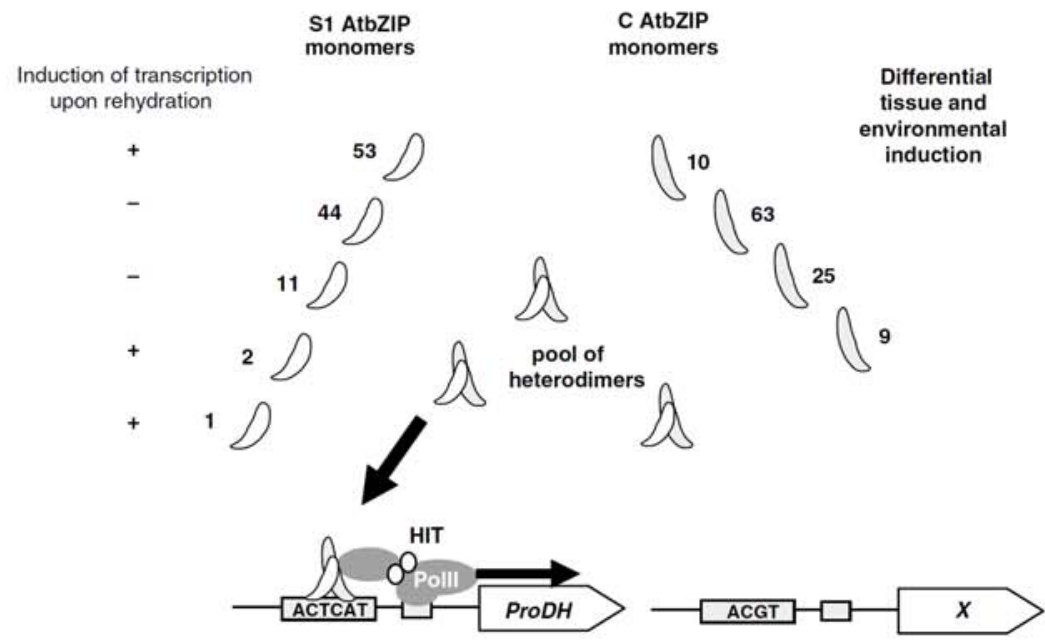

Figure 10 Model describing the combinatorial control of ProDH transcription by the network of group-S1/C heterodimers. (1) Developmenta and tissue-specific cues define expression of AtbZIP genes and consequently, the cellular amount of transcription factors. In particular. AtbZIP53, AtbZIP1, and AtbZIP2 are transcriptionally induced by environmental signals, for example, rehydration after osmotic stress (hypoosmolarity). As a consequence, colocalisation defines which heterodimers can be formed. (2) Specific group-S1 and C heterodimers are formed with high affinity before DNA binding. Therefore, a pool of specific heterodimers can be postulated to exist in the nucleus. (3) Heterodimers select target genes, such as ProDH. Moreover, group-S1/C heterodimers exhibit enhanced transactivation capacity (heterodimer-induced transactivation, HIT), which is reflected by efficient recruitment of transcriptional cofactors and subunits of the polymerase II-dependent transcription initiation complex (PolII). We therefore postulate that bZIP heterodimerisation acts as a crucial mechanism for signal integration on the level of transcription.

the ProDH gene is a direct target of the bZIP transcription factor AtbZIP53. Loss-of-function approaches show, however, that although AtbZIP53 clearly mediates hypo-osmolarityinduced $P r o D H$ transcription, it is not the only factor involved in this process, as the elimination of AtbZIP53 activity does not result in complete lack of ProDH activation. In this respect, additional data obtained in transient expression assays in Arabidopsis protoplasts suggest that the closely related group-S1 bZIP proteins might also participate in ProDH regulation (Satoh et al, 2004). In conclusion, a subset of five group-S1 bZIP factors including AtbZIP53 seems to have overlapping, partly redundant functions. As single and double knockout lines (FW, KD and WDL, unpublished results) only partly reduce $\mathrm{ProDH}$ target gene expression, multiple ko lines are needed to assess their function.

\section{HIT-a central mechanism to modulate activation properties of bZIP transcription factors in plants}

By means of yeast two-hybrid analysis, interaction studies in protoplasts and BiFC, we could establish that AtbZIP53 homodimerisation and heterodimerisation with other group$\mathrm{S}$ members is not favoured, but instead AtbZIP53 preferentially interacts with group-C bZIP proteins. A similar heterodimerisation behaviour has already been described for homologous proteins from tobacco (Strathmann et al, 2001) and parsley (Rügner et al, 2001). We have also determined that this interaction relies on the ZIP structure, as it could be abolished by introducing amino-acid exchanges that disrupt the $\alpha$-helical structure of the ZIP domain. Moreover, BiFC assays confirmed the occurrence of this preferred heterodimerisation in planta, as revealed by fluorescence due to complementation of two unfunctional parts of the YFP pro- tein fused to AtbZIP53 and AtbZIP10. The cellular localisation of YFP fusions of AtbZIP53 and AtbZIP10 could also be assigned to the nucleus, in support of a role in transcription.

While the BiFC method provides information about the cellular localisation of an interaction, it does not give quantitative information about affinity of the protein partners. To address this question, we reproduced the principle of the yeast two-hybrid system to Arabidopsis protoplasts. Basically, results obtained in the yeast system could be confirmed in planta. Moreover, this assay allows discriminating weak interactions inside the group-S1 from strong interactions between groups $\mathrm{S} 1$ and $\mathrm{C}$.

In the model plant Arabidopsis thaliana, 75 bZIP genes have been identified (Jakoby et al, 2002) and heterodimerisation has been experimentally shown for closely related bZIP proteins, like the GBFs (Schindler et al, 1992) or $\mathrm{Hy} 5 / \mathrm{HyH}$ (Holm et al, 2002). Based on data obtained for dimerisation specificity of human bZIP proteins, a model for prediction of the Arabidopsis bZIP dimerisation has been proposed (Deppmann et al, 2004). However, group-S1 and group-C interactions, which have been confirmed experimentally, have not been predicted. Dimerisation of human bZIPs has been studied experimentally by means of protein-CHIP techniques (Newman and Keating, 2003); however, bioinformatic studies using these experimental data conclude that no easy rules can be inferred for predictions (Fong et al, 2004). Hence, further experimental studies are needed to assess the complex heterodimerisation patterns in Arabidopsis. In general, group- $\mathrm{S} 1$ and $-\mathrm{C}$ have been found to form specific heterodimers (Ehlert et al, 2006). As group-S1 and -C share an unusual long ZIP domain harbouring eight repeats, we assume that the observed high-affinity interaction is due to the intrinsic structural properties of this ZIP domain. 
In vitro DNA-binding experiments show that group-S1 bZIP proteins can bind DNA without a group-C partner. This finding could be explained by monomeric DNA binding which has been described before (Metallo and Schepartz, 1997; Cranz et al, 2004). Alternatively, structural analyses of bZIP proteins have demonstrated that by DNA binding the conformation of the basic domain renders into a coiled structure which is linear with the ZIP domain ( $\mathrm{Pu}$ and Struhl, 1991). Hence, DNA-bZIP protein complex formation might alter dimerisation properties. Our experimental system makes use of the GAL4 DNA-binding domain and hence, measured protein-protein interactions are independent of DNA-binding activities. We therefore conclude that under in vivo conditions, group-S1 and $\mathrm{C}$ ZIP domains show a strong affinity without need to be associated to its target promoter DNA. It is therefore tempting to speculate that pools of group-S1/C heterodimers are formed in the nucleus, which has implications on transcriptional control of target genes (Figure 10).

The functional impact of bZIP heterodimerisation in vivo has rarely been addressed in planta. A recent publication deals with the rice bZIP factor LIP19/OBF1 in regulation of low-temperature-induced genes (Shimizu et al, 2005). In this case, heterodimerisation is postulated to modulate DNAbinding specificity. This mechanism is related to the welldescribed prototype in animals in which Fos homodimers cannot bind DNA but by heterodimerisation with Jun, another bZIP protein, the AP-1-binding site is recognised (Kouzarides and Ziff, 1988). Depending on the recruitment of bZIP heterodimerisation partners, members of the small MAF proteins can activate or repress transcription of their cognate target genes (Igarashi et al, 1994; Dlakic et al, 2001). MAF proteins are characterised by an extended DNA-binding domain and therefore guide their heterodimerisation partners to promoter binding sites which are not recognised by the bZIP on its own. In contrast to these examples, specific heterodimers of AtbZIP53 lead to strong transactivation, although neither of the single transcription factors displays significant activation potential by itself. As we have observed a comparable synergistic enhancement of transcription in a system based on ACTCAT binding and a GAL-UAS binding, this mechanism is independent of protein-DNA complex formation. These data indicate that formation of defined heterodimers generates new properties, which are relevant for gene activation under in vivo conditions. bZIP heterodimerisation might lead to the interaction with cofactors which cannot be recruited by homodimers. Alternatively, heterodimerisation might result in structural changes that might alter protein-interaction surfaces and recruitment properties. In animal systems, Jun/Fos heterodimers have been found to promote interaction with general transcription factors (GTFs) like TFIIE-34, TFIIE-30 and TFIIF-74 in vitro (Martin et al, 1996). Here, we provide conclusive evidence for the impact of a HIT mechanism in vivo. The isolation of protein-interaction partners of the group-S1/C heterodimers will give future insight into transcriptional control. In a comprehensive study, all group-C and - $\mathrm{S} 1$ interactions have been analysed, indicating that HIT is not restricted to AtbZIP53/AtbZIP10 heterodimers but is a general feature of several group-S1/C heterodimers. We therefore postulate that HIT is a general and important mechanism for fine tuning of transcription in plants.
The C/S1 network of bZIP transcription factors-signal integration on the level of transcription

A fine-tuned mechanism can be anticipated by the disclosed participation of different bZIP factors in the regulation of common target genes. In particular, here we have studied the important contribution of distinct heterodimer combinations, whose concerted action will be ultimately dependent on common localisation, protein expression levels and functional capacity to interact.

Northern analysis described in this work and web-based expression resources demonstrate that members of the $\mathrm{C} / \mathrm{S} 1$ network are transcriptionally regulated by various developmental and environmental cues. As L-Pro homeostasis has to be tightly regulated, the $\mathrm{C} / \mathrm{S} 1$ network might serve to adjust gene transcription in response to a variety of endogenous and exogenous signals. In particular, AtbZIP53, AtbZIP1, and AtbZIP2 have been shown to be activated by hypo-osmolarity (RARGE database: http://rarge.gsc.riken.jp) and promoter:GUS assays reveal that AtbZIP53 and AtbZIPIO are upregulated by hypo-osmolarity. As heterodimerisation has been shown in vivo in the nucleus independent of DNA binding to target sequences, we assume that depending on the concentration of particular bZIP proteins, pools of homoor heterodimers can be formed. These dimers compete for promoter target sites such as the ACTCAT motif in order to activate or repress transcription (Figure 10).

In the case of AtbZIP53 and AtbZIP10, it has been shown that the formation of heterodimers depends on the concentration of both partners. In addition, the amount of functionally active bZIP protein in the nucleus is regulated by various means. For instance, post-transcriptional mechanisms, like sucrose-induced repression of translation (SIRT) (Wiese et al, 2004) have been described for bZIP11/ATB2 and other S1-like bZIP genes. Nuclear translocation of AtbZIP10 has been shown to be controlled ( $\mathrm{KH}$, unpublished results) by the protein-interaction partner LSD1 (Dietrich et al, 1997). In summary, availability of bZIP heterodimerisation partners is regulated in a highly complex manner by transcriptional and post-transcriptional mechanisms.

The biological function of the members of the $\mathrm{C} / \mathrm{S} 1$ network is not well established. Recent findings provide evidence that AtbZIP10 is involved in oxidative stress response and LSD1-mediated programmed cell death $(\mathrm{KH}$, unpublished results). Accordingly, proline metabolism has been postulated to play a crucial role in cell death signalling (Deuschle et al, 2004) and very recently, a function in pathogen-induced hypersensitive response has been demonstrated (Fabro et al, 2004). We therefore assume that the C/S1 network is not only involved in recovery after osmotic stress but also represents a general regulatory circuit in protection against oxidative stress. Owing to the well-characterised promoter elements, the growing knowledge on trans acting factors and the physiological role of proline in cell-death, $\mathrm{ProDH}$ will develop as a valuable marker gene for oxidative stress responses.

Group-C bZIP factors are highly related to well-characterised bZIP proteins involved in seed-storage protein synthesis, such as $\mathrm{O} 2$ in maize (Schmidt et al, 1992). In Arabidopsis, AtbZIP10 and AtbZIP25 have been implicated in regulation of seed-storage protein genes (Lara et al, 2003). ProDH transcription is also highly controlled during seed development (Nakashima et al, 1998). Hence, we assume that besides gene regulation in oxidative stress response, the 
$\mathrm{C} / \mathrm{S} 1$ network is involved in developmentally regulated gene control.

A characteristic feature of the $\mathrm{C} / \mathrm{S} 1$ network is overlapping functional redundancy. Single ko in group-S1 or - $\mathrm{C}$ could not completely abolish $\mathrm{ProDH}$ gene activation. Heterodimerisation of group-S1 and -C bZIP factors provide an efficient mechanism to integrate signals and to adjust target gene transcription. A functional study of the bZIP proteins, the identification of target genes and a detailed expression and heterodimerisation analysis is needed to further elucidate the impact of the $\mathrm{C} / \mathrm{S} 1$ network on gene regulation in higher plants.

\section{Materials and methods}

\section{Plant material, plant treatment, plant transformation}

For protein isolation, protoplast preparation and ChIP, A thaliana ecotype Columbia (Col-O) was grown on soil under controlled environmental conditions at $16 \mathrm{~h}$ light $/ 8 \mathrm{~h}$ dark cycles. To improve germination uniformity, plants were pretreated at $4 \mathrm{C}$ for $2-4$ days. Hypo-osmolarity treatment was performed according to Satoh et al (2002). Floral dip transformations were performed by using Agrobacterium tumefaciens strains GVG3101 and wild-type A. thaliana (ecotype Col-0) (Weigel and Glazebrook, 2002).

\section{Identification of T-DNA mutants}

Seeds of the T-DNA insertion mutants atbzip10 (NASC ID: N606031) and atbzip53 (NASC ID: N569883) were obtained from the Nottingham Arabidopsis Stock Centre. Homozygous mutants were identified by PCR as described in (http://signal.salk.edu/tdnaprimers.2.html). AtbZIP53-specific primers were $5^{\prime}$-CTGCAGCTTAG GACAGCTCATCACCA-3' (AtbZIP53pGUSf) and 5'-GTCGACCTCGTT GACTTTTTGACTTC-3' (AtbZIP53pGUSr), AtbZIP10-specific primers were $5^{\prime}$-TCTTCTCCATTGACGATTTCTC-3' (AtbZIP10for) and $5^{\prime}$ TGATCTTCCGAGAAGCATCGG-3' (AtbZIP10rev), the T-DNA-specific primer was 5'-TGGTTCACGTAGTGGGCCATCG-3' (Lba1). Homozygous atbzip10 and atbzip53 T-DNA insertion mutants were crossed and in the $\{2$-generation double mutants were identified by PCR.

\section{Molecular biological techniques}

Standard DNA techniques have been described in Sambrook et al (1989). Plant RNA was isolated using TRIZOL Reagent (Life Technologies, Rockville, MD, USA), Northern analysis has been described in Heinekamp et al (2002). Hybridisation probes were produced by PCR amplification from cDNA using specifically

\section{References}

Baxevanis AD, Vinson CR (1993) Interactions of coiled coils in transcription factors: where is the specificity? Curr Opin Genet Dev 3: 278-285

Bensmihen S, To A, Lambert G, Kroj T, Giraudat J, Parcy F (2004) Analysis of an activated ABI5 allele using a new selection method for transgenic Arabidopsis seeds. FEBS Lett 561: 127-131

Cranz S, Berger C, Baici A, Jelesarov I, Bosshard HR (2004) Monomeric and dimeric bZIP transcription factor GCN4 bind at the same rate to their target DNA site. Biochemistry 43: 718-727

Delauney AJ, Hu CA, Kishor PB, Verma DP (1993) Cloning of ornithine delta-aminotransferase cDNA from Vigna aconitifolia by trans-complementation in Escherichia coli and regulation of proline biosynthesis. J Biol Chem 268: 18673-18678

Deppmann CD, Acharya A, Rishi V, Wobbes B, Smeekens S, Taparowsky EJ, Vinson C (2004) Dimerization specificity of all 67 B-ZIP motifs in Arabidopsis thaliana: a comparison to Homo sapiens B-ZIP motifs. Nucleic Acids Res 32: 3435-3445

Deuschle K, Funck D, Forlani G, Stransky H, Biehl A, Leister D, van der Graaff E, Kunze R, Frommer WB (2004) The role of [Delta]1pyrroline-5-carboxylate dehydrogenase in proline degradation. Plant Cell 16: 3413-3425

Dietrich RA, Richberg MH, Schmidt R, Dean C, Dangl JL (1997) A novel zinc finger protein is encoded by the Arabidopsis LSD1 designed primers. ProDH: $5^{\prime}$-TGGTCGGTCTTTGTCAGCAT- $3^{\prime}$ and 5'-ATGGCAACCCGTCTTCTCCG-3'; AtbZIP53: 5'-CATACAAAGACAC GAACAAATTGAA- $3^{\prime}$ and $5^{\prime}$-GTGCTTGAGATGGTTGAAGAAATTA- $3^{\prime}$; actins: $5^{\prime}$-GCTGGATTCGCTGGAGATGA-3' and $5^{\prime}$-AGGTCTCCATCT CTTGCTCG-3', respectively. A hybridisation probe for AtbZIP10 was obtained by digestion of pDONR201-AtbZIP10 with Bsp14071. Western analysis was described in Heinekamp et al (2002) using a polyclonal HA-antibody (Santa Cruz, Santa Cruz, CA, USA). Vector constructions are described in detail in Supplementary methods S1. ChIP was performed according to Johnson et al (2001) with modifications described in Supplementary methods S1.

\section{Yeast two-hybrid analysis}

ProQuest ${ }^{16}$ Y2H vectors pDEST22 and pDEST32 containing the different AtbZIP genes were transformed into the yeast strain MaV203 (Invitrogen, Karlsruhe, Germany) according to the protocol of Dohmen et al (1991). Handling of yeast cultures, plate growth assays and quantitative $\beta$-galactosidase assays were performed as described in the Clontech Yeast Protocols Handbook (1996).

\section{Protoplast transformation and GUS assays and BIFC}

Protoplast isolation and transformation was performed as described by Ehlert et al (2006). BiFC was performed in 6-week-old Nicotiana benthamiana plants after Agrobacterium-mediated transient transformation according to Walter et al (2004). For analysis of the BiFC signal, a confocal microscope (Leica TCS SP2 AOBS) was applied.

Electrophoretic mobility shift assays

EMSAs were performed as described by Lara et al (2003). The oligonucleotides PRODHS (5'-ATTCATCATCC ACTCAT CCT-3') and PRODHAS (5'-AGATATGAAGG ATGAGT GGA-3') were used as dsDNA probe.

\section{Supplementary data}

Supplementary data are available at The EMBO Journal Online.

\section{Acknowledgements}

We thank C Gatz (University of Göttingen, Germany) for constan support and for critical reading of the manuscript and T Butterbrodt (University of Göttingen, Germany) for assistance during the development of ChIP. We are grateful to B Weisshaar (University of Bielefeld, Germany), S Smeekens (University of Utrecht, The Netherlands) and T Kavanagh (University of Dublin, Ireland) for supplying plasmid constructs. This research was supported, in part, by grants from the DFG to FW (GRK242) and to KH (SFB446) and from European Community to XW (REGIA).

gene and functions as a negative regulator of plant cell death. Cell 88: 685-694

Dlakic M, Grinberg AV, Leonard DA, Kerppola TK (2001) DNA sequence-dependent folding determines the divergence in binding specificities between Maf and other bZIP proteins. EMBO J 20: $828-840$

Dohmen RJ, Strasser AW, Honer CB, Hollenberg CP (1991) An efficient transformation procedure enabling long-term storage of competent cells of various yeast genera. Yeast 7: 691-692

Ehlert A, Weltmeier F, Wang X, Smeekens S, Vicente-Carbajosa J, Dröge-Laser W (2006) Two-hybrid protein-protein interaction analysis in Arabidopsis protoplasts: establishment of a heterodimerisation map of group-C and S bZIP transcription factors. Plant J 46: 890-900

Ellenberger TE, Brandl CJ, Struhl K, Harrison SC (1992) The GCN4 basic region leucine zipper binds DNA as a dimer of uninterrupted alpha helices: crystal structure of the protein-DNA complex. Cell 71: 1223-1237

Fabro G, Kovacs I, Pavet V, Szabados L, Alvarez ME (2004) Proline accumulation and AtP5CS2 gene activation are induced by plantpathogen incompatible interactions in Arabidopsis. Mol Plant Microbe Interact 17: $343-350$

Fong JH, Keating AE, Singh M (2004) Predictng specificity in bZIP coiled-coil protein interactions. Genome Biol 5: R1 
Giniger E, Varnum SM, Ptashne M (1985) Specific DNA binding of GAL4, a positive regulatory protein of yeast. Cell 40: $767-774$

Heinekamp T, Kuhlmann M, Len A, Strathmann A, Dröge-Laser W (2002) The tobacco bZIP transcription factor BZI-1 binds to G-box promoter cis-elements of phenylpropanoid pathway genes in vitro, but it is not involved in their regulation in vivo. Mol Gen Genom 267: 16-26

Hellmann H, Funck D, Rentsch D, Frommer WB (2000) Hypersensitivity of an Arabidopsis sugar signaling mutant toward exogenous proline application. Plant Physiol 122: 357-368

Hoekstra FA, Golovina EA, Buitink J (2001) Mechanisms of plant desiccation tolerance. Trends Plant Sci 6: 431-438

Holm M, Ma LG, Qu LJ, Deng XW (2002) Two interacting bZIP proteins are direct targets of COP1-mediated control of lightdependent gene expression in Arabidopsis. Genes Dev 16: 1247-1259

Igarashi K, Kataoka K, Itoh K, Hayashi N, Nishizawa M, Yamamoto M (1994) Regulation of transcription by dimerization of erythroid factor NF-E2 p45 with small Maf proteins [see comments]. Nature 367: $568-572$

Jakoby M, Weisshaar B, Dröge-Laser W, Vicente-Carbajosa J, Tiedemann J, Kroj T, Parcy F (2002) bZIP transcription factors in Arabidopsis. Trends Plant Sci 7: 106-111

Johnson C, Boden E, Desai M, Pascuzzi P, Arias J (2001) In vivo target promoter-binding activities of a xenobiotic stress-activated TGA factor. Plant $J$ 28: 237-243

Kirby J, Kavanagh TA (2002) NAN fusions: a synthetic sialidase reporter gene as a sensitive and versatile partner for GUS. Plant $J$ 32: $391-400$

Kiyosue T, Yoshiba Y, Yamaguchi-Shinozaki K, Shinozaki K (1996) A nuclear gene encoding mitochondrial proline dehydrogenase, an enzyme involved in proline metabolism, is upregulated by proline but downregulated by dehydration in Arabidopsis. Plant Cell 8: 1323-1335

Kouzarides T, Ziff E (1988) The role of the leucine zipper in the fos-jun interaction. Nature 336: 646-651

Landschulz WH, Johnson PF, McKnight SL (1988) The leucine zipper: a hypothetical structure common to a new class of DNA binding proteins. Science 240: 759-764

Lara P, Onate-Sanchez L, Abraham Z, Ferrandiz C, Diaz I, Carbonero P, Vicente-Carbajosa J (2003) Synergistic activation of seed storage protein gene expression in Arabidopsis by $\mathrm{ABI} 3$ and two bZIPs related to OPAQUE2. J Biol Chem 278: 21003-21011

Liu J, Zhu JK (1997) Proline accumulation and salt-stress-induced gene expression in a salt-hypersensitive mutant of Arabidopsis. Plant Physiol 114: 591-596

Mansour M (1998) Protection of plasma membrane of onion epidermal cells by glycinebetaine and proline against $\mathrm{NaCl}$ stress. Plant Physiol Biochem 36: 767-772

Martin ML, Lieberman PM, Curran T (1996) Fos-Jun dimerization promotes interaction of the basic region with TFIIE-34 and TFIIF. Mol Cell Biol 16: 2110-2118

Metallo SJ, Schepartz A (1997) Certain bZIP peptides bind DNA sequentially as monomers and dimerize on the DNA. Nat Struct Biol 4: 115-117

Naar AM, Lemon BD, Tjian R (2001) Transcriptional coactivator complexes. Annu Rev Biochem 70: 475-501

Nakashima K, Satoh R, Kiyosue T, Yamaguchi-Shinozaki K, Shinozaki K (1998) A gene encoding proline dehydrogenase is not only induced by proline and hypoosmolarity, but is also developmentally regulated in the reproductive organs of Arabidopsis. Plant Physiol 118: 1233-1241

Nanjo T, Fujita M, Seki M, Kato T, Tabata S, Shinozaki K (2003) Toxicity of free proline revealed in an Arabidopsis T-DNA-tagged mutant deficient in proline dehydrogenase. Plant Cell Physiol 44: 541-548

Nanjo T, Kobayashi M, Yoshiba Y, Kakubari Y, Yamaguchi-Shinozaki K, Shinozaki K (1999) Antisense suppression of proline degradation improves tolerance to freezing and salinity in Arabidopsis thaliana. FEBS Lett 461: 205-210

Newman JR, Keating AE (2003) Comprehensive identification of human bZIP interactions with coiled-coil arrays. Science 300: 2097-2101

Oono Y, Seki M, Nanjo T, Narusaka M, Fujita M, Satoh R, Satou M, Sakurai T, Ishida J, Akiyama K, lida K, Maruyama K, Satoh S, Yamaguchi-Shinozaki K, Shinozaki K (2003) Monitoring expression profiles of Arabidopsis gene expression during rehydration process after dehydration using ca 7000 full-length cDNA microarray. Plant $J$ 34: 868-887

Parvanova D, Ivanov S, Konstantinova T, Karanov E, Atanassov A Tsvetkov T, Alexieva V, Diilianov D (2004) Transgenic tobacco plants accumulating osmolytes show reduced oxidative damage under freezing stress. Plant Physiol Biochem 42: 57-63

Peng Z, Lu Q, Verma DP (1996) Reciprocal regulation of delta 1-pyrroline-5-carboxylate synthetase and proline dehydrogenase genes controls proline levels during and after osmotic stress in plants. Mol Gen Genet 253: 334-341

Pu WT, Struhl K (1991) The leucine zipper symmetrically positions the adjacent basic regions for specific DNA binding. Proc Natl Acad Sci USA 88: 6901-6905

Rentsch D, Hirner B, Schmelzer E, Frommer WB (1996) Salt stress. induced proline transporters and salt stress-repressed broad specificity amino acid permeases identified by suppression of a yeas amino acid permease-targeting mutant. Plant Cell 8: 1437-1446

Roosens NH, Willem R, Li Y, Verbrüggen II, Biesemans M, Jacobs M (1999) Proline metabolism in the wild-type and in a salt-tolerant mutant of Nicotiana plumbaginifolia studied by (13)C-nuclear magnetic resonance imaging. Plant Physiol 121: 1281-1290

Rügner A, Frohnmeyer H, Nake C, Wellmer F, Kircher S, Schäfer E, Harter K (2001) Isolation and characterization of four novel parsley proteins that interact with the transcriptional regulators CPRF1 and CPRF2. Mol Genet Genomics 265: 964-976

Sambrook J, Fritsch EF, Maniatis T (1989) Molecular Cloning: A Laboratory Manual. Cold Spring Habour, New York, USA: Cold Spring Harbour Laboratory Press

Satoh R, Fujita Y, Nakashima K, Shinozaki K, Yamaguchi-Shinozaki $K$ (2004) A novel subgroup of bZIP proteins functions as transcriptional activators in hypoosmolarity-responsive expression of the ProDH gene in Arabidopsis. Plant Cell Physiol 45: 309-317

Satoh R, Nakashima K, Seki M, Shinozaki K, Yamaguchi-Shinozaki K (2002) ACTCAT, a novel cis-acting element for proline- and hypoosmolarity-responsive expression of the ProDH gene encoding proline dehydrogenase in Arabidopsis. Plant Physiol 130: 709-719

Schindler U, Menkens AE, Beckmann H, Ecker JR, Cashmore AR (1992) Heterodimerization between light-regulated and ubiquitously expressed Arabidopsis GBF bZIP proteins. EMBO J 11: 1261-1273

Schmidt RJ, Ketudat M, Aukerman MJ, Hoschek G (1992) Opaque-2 is a transcriptional activator that recognizes a specific target site in 22-kD zein genes. Plant Cell 4: 689-700

Shimizu H, Sato K, Berberich T, Miyazaki A, Ozaki R, Imai R, Kusano T (2005) LIP19, a basic region leucine zipper protein, is a Fos-like molecular switch in the cold signaling of rice plants. Plant Cell Physiol 46: 1023-1043

Smirnoff N, Cumbes QJ (1989) Hydroxyl radical scavenging activity of compatible solutes. Phytochemistry 28: 1057-1060

Sprenger-Haussels M, Weisshaar B (2000) Transactivation properties of parsley proline-rich bZIP transcription factors. Plant $J \mathbf{2 2}$ $1-8$

Strathmann A, Kuhlmann M, Heinekamp T, Dröge-Laser W (2001) BZI-1 specifically heterodimerises with the tobacco bZIP transcription factors BZI-2, BZI-3/TBZF and BZI-4, and is functionally involved in flower development. Plant $J$ 28: 397-408

Verbrüggen N, Hua XJ, May M, Van Montagu M (1996) Environmental and developmental signals modulate proline homeostasis: evidence for a negative transcriptional regulator. Proc Natl Acad Sci USA 93: 8787-8791

Walter M, Chaban C, Schutze K, Batistic O, Weckermann K, Nake C. Blazevic D, Grefen C, Schumacher K, Oecking C, Harter K, Kudla J (2004) Visualization of protein interactions in living plant cells using bimolecular fluorescence complementation. Plant J 40: 428-438

Walton EF, Boldingh HL (1991) Effects on hydrogen cyanamide on amino acid profiles in kiwifruit buds during budbreak. Plant Physiol 97: 1256-1259

Weigel R, Glazebrook J (2002) Arabidopsis: A Laboratory Manual. Cold Spring Habour, New York, USA: Cold Spring Harbour Laboratory Press

Wiese A, Elzinga N, Wobbes B, Smeekens S (2004) A conserved upstream open reading frame mediates sucrose-induced repression of translation. Plant Cell 16: 1717-1729

Yoshiba Y, Nanjo T, Miura S, Yamaguchi-Shinozaki K, Shinozaki K (1999) Stress-responsive and developmental regulation of Delta(1)-pyrroline-5-carboxylate synthetase 1 (P5CS1) gene expression in Arabidopsis thaliana. Biochem Biophys Res Commun 261: 766-772 


\title{
6 Heterodimers of C/S1 bZIP network controls gene expression in low energy stress response
}

\section{Heterodimers of the Arabidopsis Transcription Factors bZIP1 and bZIP53 are Reprogramming Amino Acid Metabolism during Low Energy Stress}

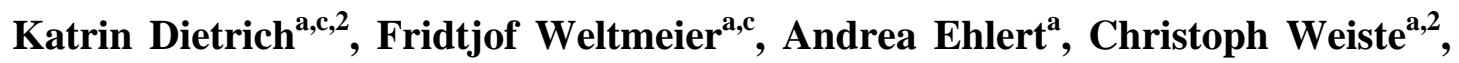 \\ Mark Stahl $^{\mathrm{b}}$, Klaus Harter ${ }^{\mathrm{b}}$, Wolfgang Dröge-Laser ${ }^{\mathrm{a}, 1,2}$ \\ ${ }^{a}$ Albrecht-von-Haller-Institut, Universität Göttingen, Untere Karspüle 2, D-37073 Göttingen, Germany \\ ${ }^{\mathrm{b}}$ Zentrum für Molekularbiologie der Pflanzen, Pflanzenphysiologie, Auf der Morgenstelle 1, 72076 \\ Tübingen, Germany \\ ${ }^{1}$ To whom correspondence should be addressed: \\ Wolfgang Dröge- Laser, email: wolfgang.droege-laser@uni-wuerzburg.de \\ ${ }^{2}$ Authors new location: Julius-von-Sachs-Institut, Pharm. Biol., Universität Würzburg \\ Julius-von-Sachs-Platz 2, D-97082 Wüezburg
}

\section{SUMMARY}

Control of energy homeostasis is crucial for plant survival, in particular when facing biotic or abiotic stress conditions. Energy deprivation induces dramatic reprogramming of transcription facilitating metabolic adjustment. The in-depth knowledge of the corresponding regulatory networks provides opportunities for the development of future biotechnology strategies. Low energy stress activates the Arabidopsis group $\mathrm{S} 1$ basic leucine zipper transcription factors bZIP1 and bZIP53 by transcriptional and posttranscriptional mechanisms. Gain-of-function approaches define these bZIPs as crucial transcriptional regulators in proline, asparagine and branchedchain amino acid metabolism. Whereas ChIP analyses confirm the direct binding of bZIP1 and bZIP53 to promoters of key metabolic genes such as ASPARAGINE SYNTHETASE (ASN1) and PROLINE DEHYDROGENASE
(ProDH), the G-box, C-box or ACT-motives (ACTCAT) have been defined as regulatory cis-elements in the starvation response. bZIP1 and bZIP53 were shown to specifically heterodimerise with group C bZIPs. Although single loss-of-function mutants did not effect starvationinduced transcription, quadruple mutants of group S1 and C bZIPs displayed a significant impairment. We therefore propose, that bZIP1 and bZIP53 transduce low energy signals by heterodimerisation with members of the partially redundant $\mathrm{C} / \mathrm{S} 1 \mathrm{bZIP}$ factor network to reprogram primary metabolism in starvation response.

\section{INTRODUCTION}

Due to their photothrophic life style, plants have to steadily adjust their metabolism to daynight rhythms and environmental changes to accomplish transient energy deprivation (for review see Baena-Gonzalez and Sheen, 2008; Usadel et al., 2008). Low energy stress can easily be mimicked by the cultivation of plants 
in the dark. Extended dark-treatment is correlated with dramatic changes in primary plant metabolism in particular reduced photosynthesis, degradation of proteins, amino acids (aa) or nucleic acids, hydrolysis of polysaccharides or oxidation of fatty acids. These physiological changes are accompanied by a massive reprogramming of transcription which is reflected in several recent transcriptome profiling studies (Gan, 2003; Buchanan-Wollaston et al., 2005; Lin and Wu, 2004) In particular, the whole set of genes leading to the biosynthesis of asparagine (Asn) is reprogrammed in response to dark treatment. In comparison to glutamine (Gln), the major transport form of nitrogen in the light, Asn contains less carbon than Gln and is therefore used to store and transport nitrogen especially under stress conditions where carbon is limited (Lam et al., 1994). Recently, the Arabidopsis thaliana SnRK1-like kinases (SNF1-related protein kinases 1) KIN10 and KIN11 have been proposed to function as central signalling integrators mediating adaptation to low energy stress (Baena-Gonzalez et al., 2007). These kinases show structural similarities to the SNF1-kinase (SUCROSE NONFERMENTING 1) in yeast and the AMPK (AMP-DEPENDENT PROTEIN KINASE) in mammals which function as master regulators of the energy balance essential for survival under stress (Polge and Thomas, 2007). In Arabidopsis, the ASPARAGINE SYNTHETASE gene (ASN1) which encodes the final step in Asn biosynthesis was proposed to be a target of the KIN10/11 pathway controlling the level of Asn (Baena-Gonzalez et al., 2007). The dark- or stress-induced regulation of ASN1 gene expression is mediated specifically by a G-box ciselement (Baena-Gonzalez et al., 2007; Hanson et al., 2008), typically recognized by basic leucine zipper (bZIP) transcription factors (TFs). bZIP proteins, exclusively found in eukaryotic cells, bind DNA by forming homo- or heterodimers. In the Arabidopsis genome, 75 bZIP genes have been identified and classified into 10 groups (Jakoby et al., 2002). Interestingly, only a specific subset of G-box binding bZIP factors was shown to activate ASN1 in transiently transformed protoplasts (BaenaGonzalez et al., 2007), namely bZIP2 (GBF5, At2g18160), bZIP11 (ATB2, At4g34590), bZIP53 (At3g62420) and bZIP1 (At5g49450). Based on aa homology and specific heterodimerisation properties with group $\mathrm{C}$ bZIPs these proteins were classified as $\mathrm{S} 1 \mathrm{sub}$ group (Ehlert et al., 2006). These C and S1 bZIPs form a functional interlinked $\mathrm{TF}$ network (Weltmeier et al., 2009). In a transcriptome analysis using plants expressing bZIP11 in a dexamethasone inducible manner, ASN1 was shown to be regulated by bZIP11 (Hanson et al., 2008). Furthermore, bZIP53 regulates the expression of PROLINE DEHYDROGENASE (ProDH) during the hypoosmolarity response (Nakashima et al., 1998; Satoh et al., 2004; Weltmeier et al., 2006). ProDH degrades the compatible osmolyte proline during recovery from stress. To deal with diurnal changes in carbon supplies, plants retain some photosynthates as starch which can be remobilized during the night (Usadel et al., 2008). However, within 2 - 4 hours of an extended night, these resources are depleted leading to severe limitation of carbohydrates. This metabolic process is demonstrated in the starchless pgm mutant from Arabidopsis, which uses up their carbohydrate resources within the first few hours of night eventually leading to growth retardation (Usadel et al., 2008). Expression profiling of plants cultivated in an extended night regime allowed to build-up regulatory models proposing that they respond to small changes in the carbon status in an acclimatory manner (Usadel et al., 2008). In this work, several group S1 bZIPs, including bZIP1 and bZIP53 were suggested to be involved in the plants response to carbohydrate starvation. Accordingly, systems biology approaches studying the integration of $\mathrm{C}$ and $\mathrm{N}$ derived metabolic signals proposed bZIP1 as a regulator in the nitrogen-responsive gene 
network which includes the modulation of ASN1 gene expression (Gutierrez et al., 2008). Although several members of the group S1 bZIPs have been implicated in starvation responses and particularly in aa metabolism, experimental data are limited to gain-offunction studies in plant protoplasts. Here, we define bZIP1 and, to a minor extent, bZIP53 as transcriptionally and post-transcriptionally activated TFs in the low energy stress response of Arabidopsis. The impact of bZIP1 and bZIP53 on starvation-induced transcription of key genes in the aa metabolism and aa accumulation is demonstrated in protoplasts and transgenic plants. The results of loss-offunction approaches indicate that several, partially redundant TFs of the C/S1 bZIP network co-operate to control plant low energy responses.

\section{RESULTS}

\section{bZIP1 and bZIP53 expression is enhanced during dark-induced starvation}

In order to identify candidate bZIP TFs, which are involved in regulating plant starvation responses, a screening of public expression databases and quantitative real-time PCR (qRT-PCR) using RNA from plants exposed to extended darkness was performed. The expression of bZIP1 was strongly induced upon extended dark treatment and repressed by sugars (Supplemental Figure S1A,B,C online). A minor but reproducible transcriptional induction was also observed for the closest bZIP1 homologue, bZIP53, but not for the other group S1 bZIPs. Based on these findings, bZIP1 and bZIP53 were selected as candidate transcriptional regulators to study the darkinduced starvation response of Arabidopsis. To further substantiate these findings, a detailed time course expression experiment was performed. Remarkably, a night extension up to 4 hours leads to an 8 fold accumulation of bZIP1 transcripts (Figure 1A) which further increased during extended night up to 30 fold. bZIP53 transcripts accumulate only slightly during extended night (3-4 fold). Histochemical stainings of plants containing promoter reporter constructs (ProbZIP1:GUS, ProbZIP53:GUS) were used to demonstrate bZIP expression on whole plant level. Whereas the GUS staining of plants grown under a 16/8h day-night cycle demonstrated a bZIP1 and bZIP53 gene activity only in young sink leaves (Weltmeier et al., 2009), the prolonged incubation in the dark led to a rapid spreading of expression patterns also in older, welldeveloped source leaves (Figure 1B). However, this response was not detected in transgenic lines harbouring promoter:reporter constructs of other group S1 bZIPs (e.g. bZIP11 or bZIP44) (Weltmeier et al., 2009). As demonstrated in previous studies (Wiese et al., 2004; Weltmeier et al., 2009), a posttranscriptional regulatory mechanism applies for all group S1 bZIPs including bZIP1 and bZIP53 which leads to a sucrose-induced repression of translation (SIRT) mediated by a conserved system of upstream open reading frames (uORFs). With respect to bZIP1 and bZIP53, transcriptional and posttranscriptional mechanisms interact to enhance expression in response to dark treatment. In contrast, in particular bZIP11 shows an inverse regulation. Transcription is repressed by dark treatment and induced by sugar application (Supplemental Figure S1B online). Hence, these differences in expression propose a function for bZIP1 and bZIP53 in the darkinduced starvation response which is not shared by the other group S1 members.

\section{Ectopic expression of bZIP1 and bZIP53 results in enhanced dark-induced senescence}

To further study the function of bZIP1 and bZIP53 in the dark-induced starvation response, the phenotypes of plants ectopically expressing bZIP53 and bZIP1 
A

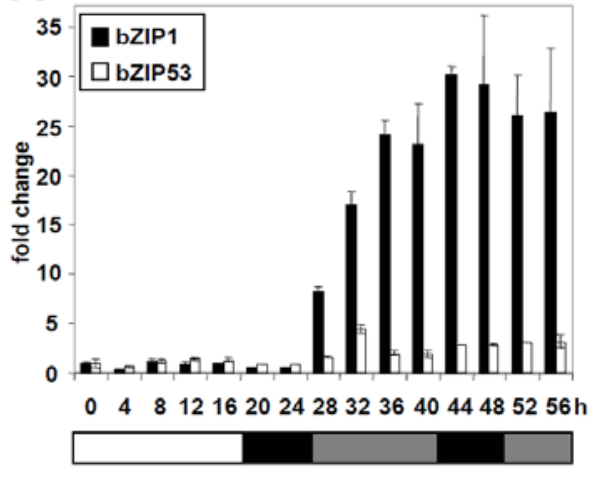

B
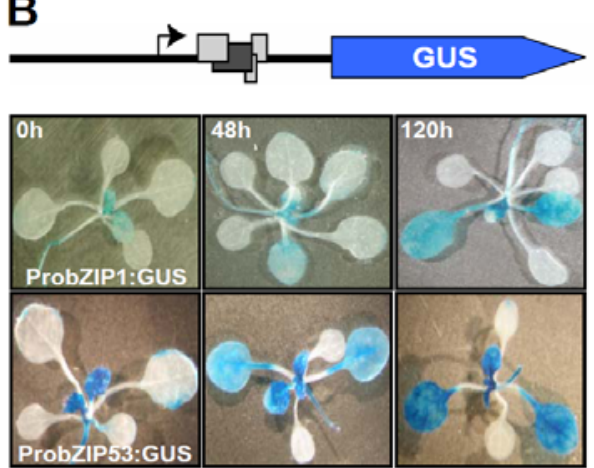

C

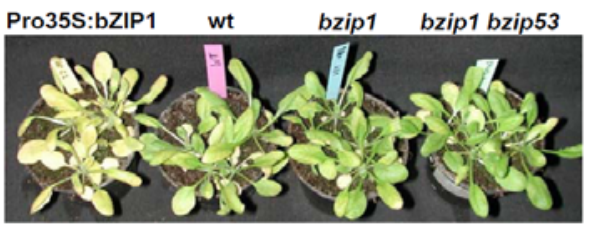

D

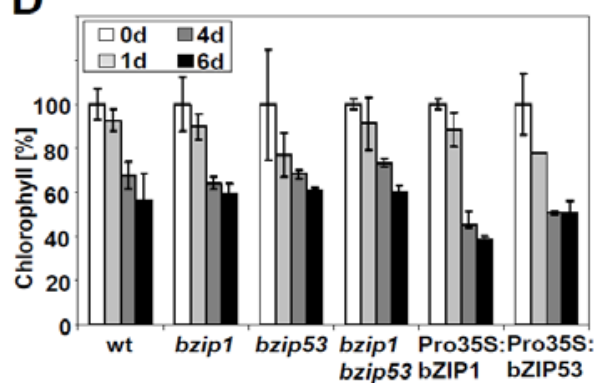

Figure 1. Analysis of bZIP1 and bZIP53 in low energy response (A) Expression of bZIP1 and bZIP53 increases after extended night treatment. Wildtype (wt) plants are cultivated at a day/night cycle of $16 / 8 \mathrm{~h}$ as indicated by the scheme. Day, night and extended night phases are indicated by white, black or grey bars, respectively. Transcript abundance as determined by qPCR has been presented for bZIP1 (black bars) and bZIP53 (white bars) depicted as fold induction. (B) Histochemical GUS staining of transgenic plants expressing ProbZIP1:GUS (upper panel) and ProbZIP53:GUS (lower panel). The upstream regions contain the conserved system of uORFs (depicted by rectangles) which has been shown to mediate a sucrose dependent post-translational repression (Wiese et al., 2004; Weltmeier et al., 2009). GUS staining of plants grown under $16 / 8 \mathrm{~h}$ day/night cycle $(0 \mathrm{~h})$ or darkness for $48 \mathrm{~h}$ and $120 \mathrm{~h}$ are given, respectively. (C) 3-weekold plants expressing HA-tagged bZIP1 or bZIP53 under control of the 35S promoter (Pro35S:bZIP1, Pro35S:bZIP53), wt, bzip1, bzip53 as well as the corresponding double mutant (bzip1bzip53) show an enhanced senescence phenotype in the dark after 6d. (D) Relative chlorophyll content of rosette leaves of plants depicted in (C) cultured in normal day/night cycle (white bars) or for extended dark treatment as indicated.
(Pro35S:bZIP53, Pro35S:bZIP1) and their HAtagged versions (Pro35S:HAbZIP53, Pro35S:HA-bZIP1) under the control of the $35 \mathrm{~S}$ promoter were analysed (Weltmeier et al., 2006; Weltmeier et al., 2009; Supplemental Figure S2 online). Whereas Pro35S:HA-bZIP1 plants grew normal under standard day-night cycles, Pro35S:HA-bZIP53 plants showed a dwarf growth phenotype depending on the expression level of the transgene (Alonso et al., 2009). Prolonged cultivation of the bZIP overexpressing lines in the dark resulted in an obvious phenotype (Figure 1C). In particular the Pro35S:bZIP1 plants showed a faster darkinduced leaf yellowing. Accordingly, the bZIP1 and bZIP53 overexpressing plants had significantly reduced amounts of chlorophyll after 4-6 days of cultivation in darkness (Figure 1D). However, bzip1 and bzip53 single and double mutants (Supplemental Figure S2 online) did not show obvious alterations in comparison to the wild type (wt). The culture conditions were further analysed by using well-defined marker genes for ongoing leaf senescence (Supplemental Figure S3 online). Whereas the Chlorophyll A/B binding Protein gene $(\mathrm{CAB})$ - a light-induced marker for photosynthetically active leaves was transcriptionally down-regulated in darkness (van der Graaff et al., 2006), the SENESCENCE ASSOCIATED GENE 103 (SAG103), a marker for dark-induced senescence, is induced in wt plants after $24 \mathrm{~h}$ of extended night ( $48 \mathrm{~h}$ timepoint). In contrast, the YELLOW LEAF SPECIFIC (YLS3) gene, a marker for natural senescence (van der Graaff et al., 2006), was not significantly affected in its transcription. We therefore conclude that the process observed during extended night is distinct from natural senescence and that ectopic expression of bZIP1 or bZIP53 enhances physiological responses which are correlated to dark-induced starvation.

bZIP1 and bZIP53 regulate ProDH transcript level and proline content during dark-induced starvation 
ProDH which encodes an enzyme mediating the catabolism of proline (Figure 2A) is a direct transcriptional target of bZIP53 in the hypoosmolarity response of Arabidopsis (Weltmeier et al., 2006). ProDH transcription was also induced after dark treatment, as demonstrated by Northern analysis (Figure 2B) and qRT-PCR (Figure 2C). These data are in line with the hypothesis that, during starvation response, aa are recycled to support $\mathrm{C}, \mathrm{N}$ and energy demands. Ectopic expression of bZIP1 and bZIP53 resulted in significant higher levels of ProDH transcripts. However, whereas bZIP53 overexpression led to high ProDH transcript level both in light and darkness, which was further enhanced by extended dark treatment, the regulation by bZIP1 differed as the ProDH transcript accumulation was preferentially enhanced in the dark (Figure 2B,C). In addition to an overexpression analysis, we also performed loss-of-function studies using T-DNA insertion mutants of bZIP1 and bZIP53 (Supplemental Figure S2, S4 online). In contrast to single bzip1 and bzip53 mutants (Supplemental Figure S5B online), a moderate but significant reduction in the dark-induced activation of ProDH transcript accumulation was observed in bzip1 bzip53 double mutants when compared to wt (Figure 2C, Supplemental Figure S5B online). However, because the ProDH transcript level is still responsive to dark-induced starvation, additional, partly redundant transcriptional regulators have to be postulated. As the ProDH enzyme is mediating proline degradation, its activation should result in reduced Pro levels, which indeed was observed after transfer of wt plants to darkness (Figure 2D). Compared to wt and also the bzip1 bzip53 double mutant, the proline levels are significantly reduced in Pro35S:bZIP53 and Pro35S:bZIP1 plants (Figure 2D). This observation is in agreement with our postulated function of bZIP1 and bZIP53 in the ProDH-mediated proline degradation during the dark-induced starvation response.

\section{Dark-induced bZIP1 expression depends on sugar depletion}

To elucidate whether depletion of sugars, which function as the major energy resource during night, or the absence of light are acting as important regulatory signals in the expression of bZIP1, bZIP53 and ProDH, we grew Arabidopsis plants in a hydroponic culture system under different carbohydrate regimes. As shown in Figure 2E, the transcripts of bZIP1, bZIP53 and ProDH coordinately accumulated after $24 \mathrm{~h}$ in the dark. However, transcriptional regulation of bZIP53 was always less pronounced when compared to bZIP1 (Figure 1A, 2E). The plants were then transferred to medium supplemented with equimolar concentrations of 3-ortho-methylglucose (3-oMG), glucose, sucrose or polyethylenglycol (PEG) and further kept in darkness. 3-oMG serves as a control as it is taken up by the cells but does not trigger the glucose-specific sugarsignalling pathways (Cortes et al., 2003). Sucrose and glucose, but not 3-oMG repressed bZIP1, bZIP53 as well as ProDH transcript accumulation. These data suggest that sugar signalling and not the absence of light controls the transcript accumulation of bZIP1 and bZIP53. As a putative target, the ProDH transcript level followed that of the two bZIP TFs with a slower kinetic as demonstrated by comparing the $1 \mathrm{~h}$ and $2 \mathrm{~h}$ time points. It has been reported, that changing of osmolarity conditions also modulates the ProDH transcript levels (Satoh et al., 2004; Weltmeier et al., 2006). Hyperosmolarity conditions applied by PEG1000 treatment lead to down-regulation of ProDH but did not affect bZIP1 transcript accumulation. From these data we conclude that, while ProDH transcript accumulation is controlled by several different stimuli, darkinduced energy starvation results in a bZIP1/bZIP53 dependent induction of proline degradation. 

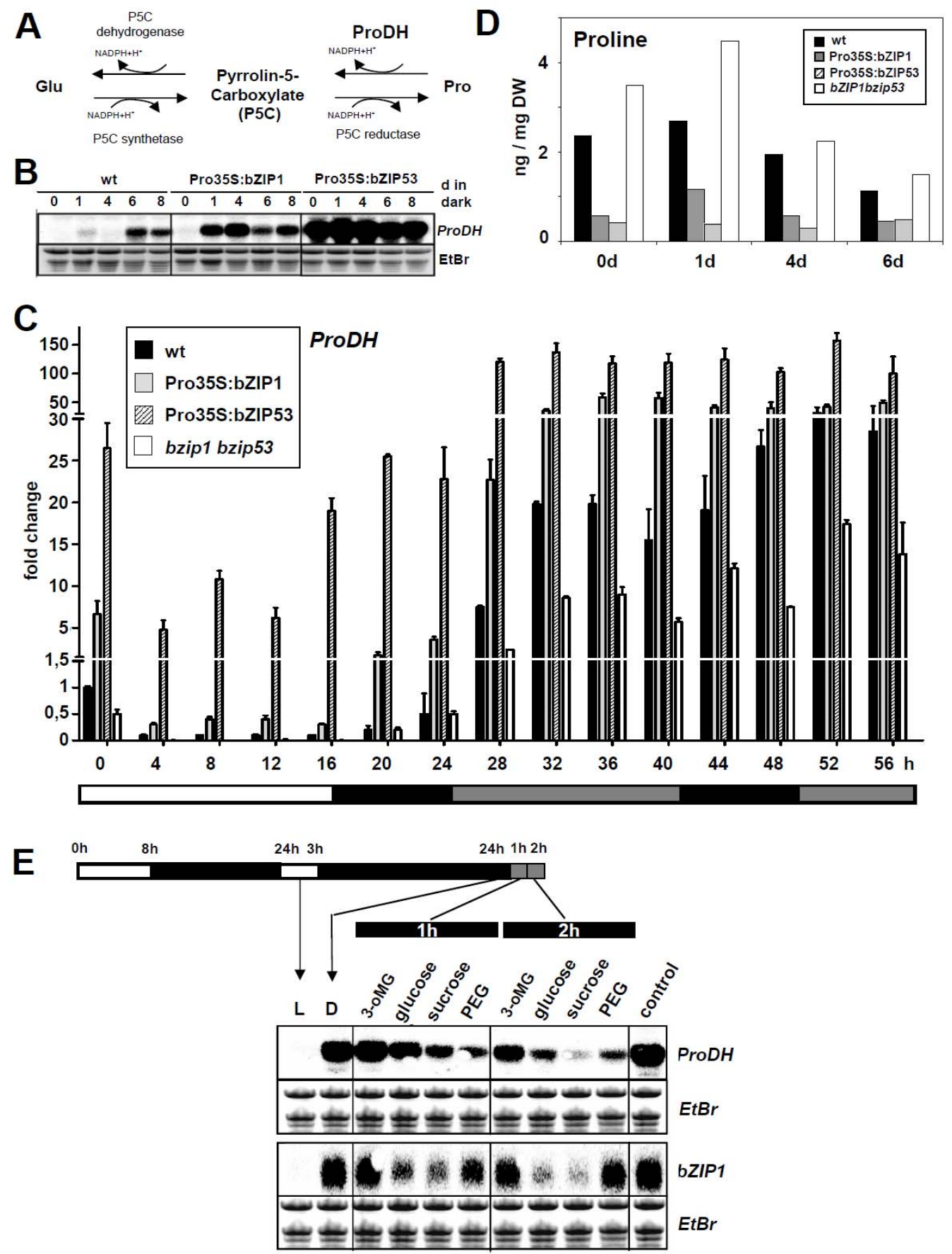

Figure 2. bZIP1 and bZIP53 control ProDH transcription and Pro content during dark-treatment. (A) The ProDH enzyme regulates catabolism of the aa Pro to Pyrrolin-5-carboxylate (P5C). Given is the complementary biosynthesis pathway based on Glu and making use of P5C as intermediate (Hellmann et al., 2000). (B) Northern analysis of ProDH in wt, Pro35S:bZIP1 (line C) and Pro35S:bZIP53 (line 10) (Weltmeier et al., 2006) in response to long-term dark treatment for $1-8 \mathrm{~d}$. (C) Induction of ProDH after short-term dark treatment analysed by qPCR as described in Figure 1A. wt (black bars), Pro35S:bZIP1 (grey bars), Pro35S:bZIP53 (hatched bars), bzip1bzip53 (white bars). For visualizing the differences in transcript levels, the y axes is broken twice at 1.5 and 30 fold induction. (D) Quantification of Pro levels in wt (black bars), Pro35S:bZIP1 (grey bars), Pro35S:bZIP53 (hatched bars) and bzip1bzip53 (white bars) after 0, 1, 4, $6 \mathrm{~d}$ of dark treatment. (E) The transcript abundance of bZIP1 is controlled by sugar depletion. 3-week old wt plants were cultivated in a hydroponic culture as depicted in the scheme. After $3 \mathrm{~h}$ in light (L) and after an additional $24 \mathrm{~h}$ in darkness (D) RNA was isolated. The plants were transferred to media containing equal molar (167mM) 3-ortho-methyl glucose (3-oMG), glucose, sucrose or PEG1000, respectively. Culture on non-supplemented media has been used as control. RNA isolated from the differently supplemented cultures after 1 or $2 \mathrm{~h}$, respectively. Given are Northern analysis of ProDH and bZIP1 transcripts. EtBr: Loading is controlled by Ethidium bromide staining. 
bZIP1 and bZIP53 regulate the level of asparagine and the branched-chain amino acids leucine, isoleucine and valine

To determine whether bZIP1 and bZIP53 regulate the aa metabolism in general during the dark-induced starvation response, a comprehensive aa analysis was performed. In wt plants, the total amount of aa increased in response to prolonged darkness. In contrast, Pro35S:bZIP53 plants showed a significantly stronger accumulation of total aa whereas in the bzip1 bzip53 double mutant the increase was less pronounced (Figure 3A). During extended night, an increase was observed especially for the levels of the Branched-Chain Amino Acids (BCAA) leucine (Leu), isoleucine (Ile) and valine (Val) as well as for Asn (Figure 3B; Supplemental Table S1 online). The increase in BCAA levels, in particular Leu and Ile, was strongly repressed in the Pro35S:bZIP1 and Pro35S:bZIP53 plants, indicating that the bZIP regulators promote the degradation of Leu and Ile. The Val levels followed a similar accumulation pattern, however it was less pronounced (Figure 3B; Supplemental Table S1 online). The impact of bZIP1 and bZIP53 on Asn metabolism differed considerably from that of Leu, Ile and Val. Dark-induced Asn levels were enhanced by the overexpression of bZIP53 but not bZIP1, whereas the bzip1 bzip53 plants displayed a slightly reduced amount of Asn (Figure 3B; Supplemental Table S1 online). Altogether, our data suggest that bZIP1 and bZIP53 participate to the transcriptional reprogramming of the aa metabolism during the dark-induced starvation response.

\section{bZIP1 and bZIP53 control the expression of genes involved in the darkinduced synthesis of asparagine and branched chain amino acids}

As bZIP1 and bZIP53 are responsible for modulation of aa levels, we tested the expression of genes which are related to the corresponding metabolic pathways. Asn is the major source for the N/C transport in darkness (Lam et al., 1994; Lam et al., 1998; Lam et al., 2003). Asn biosynthesis is derived from pyruvate and requires the coordinated, transcriptional up-regulation of several genes (Lin and $\mathrm{Wu}, 2004$; Figure 4A). Expression of these genes during dark-induced starvation was substantiated by public expression data (Supplemental Figure S6A online) and confirmed by Northern blot analysis (Figure 4B). The tested genes encoding enzymes of the Asn biosynthetic pathway such as GLUTAMATE DEHYDROGENASE (GDH2) ASPARTATE AMINOTRANSFERASE 3 (ASP3), GLUTAMATE SYNTHASE (GLNS) and ASN1 were induced during 8 days of darktreatment (Figure 4B). The overexpression of bZIP1 resulted in an enhanced or more rapid transcript accumulation of these biosynthetic genes, whereas overexpression of bZIP53 caused constitutively high transcript levels. The PepCK transcript accumulation, which encodes PEP CARBOXYKINASE, the first enzymatic step in the Asn biosynthesis, was neither induced by darkness nor by bZIP1 overexpression. A slight accumulation of PepCK transcript was only observed when bZIP53 was overexpressed (Figure 4B). A detailed time course of an extended night treatment was carried out for the central Asn biosynthesis pathway gene ASN1. Comparable to ProDH, rapid induction of the ASN1 transcript accumulation was detected within 4 $\mathrm{h}$ of the extended night treatment (Figure 4C). This response was strongly enhanced in Pro35S:bZIP1 plants but not in Pro35S:bZIP53 plants. These expression data seem to contradict the metabolic analysis because the Asn levels were higher in Pro35S:bZIP53 than in Pro35S:bZIP1 plants. However, in contrast to bZIP53, bZIP1 also activated an ASPARAGINASE gene (ANS, At3g16150) (Figure 4D), which participates to the degradation of Asn (Bruneau et al., 2006). Our observations suggest that bZIP1 and bZIP53 have partly overlapping but also distinct functions in the regulation of the Asn 
metabolism. Ectopic expression of bZIP1 and bZIP53 leads to reduced levels of BCAA, indicating their involvement in the corresponding catabolic pathway. The mitochondrial BCAA TRANSAMINASE 1 (BCAT1) gene was proposed to encode the central catabolic enzyme (Diebold et al., 2002; Schuster and Binder, 2005; Supplemental Figure S6A,B online). However, the analysis of the six Arabidopsis BCAT genes revealed, that BCAT2 and to a minor extend BCAT1 were induced during dark-treatment. As depicted in Supplemental Figure S6 online, bZIP1 strongly enhances BCAT2 transcript accumulation in the dark. The BCAT2 enzyme is localised in the chloroplasts, where it contributes to Leu and Glu biosynthesis (Schuster and Binder, 2005). Therefore, the plastidic deamination reaction of BCAT2 in the dark might supply the cell with Glu which in turn is essential for Asn biosynthesis (Supplemental Figure S6B online). In conclusion, the dark-induced accumulation of BCAT2 transcript is rather linked to the darkinduced Asn biosynthesis than to darkinduced BCAA degradation.

Promoters of amino acid metabolic genes are regulated by bZIP1 and bZIP53 in response to energy starvation in protoplasts

To assess the direct impact of bZIP1 and bZIP53 on gene regulation, the activity of ASN1 and ProDH promoter:reporter constructs (ProASN1:GUS; ProProDH:GUS) was studied in transiently transfected protoplasts. Starvation was induced by either the transfer of light-cultivated protoplasts to darkness or treatment of light-cultivated protoplasts with the photosystem II inhibitor DCMU (3-(3,4Dichlorphenyl)-1,1-dimethylurea). Both starvation treatments induced the transcriptional activity of the ProASN1:GUS and ProProDH:GUS reporter genes demonstrating that the protoplast system can be used to analyse starvationinduced transcription (Supplemental Figure S7 online).

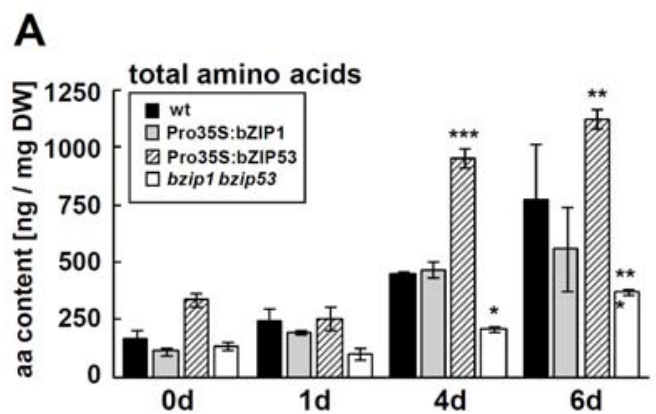

B
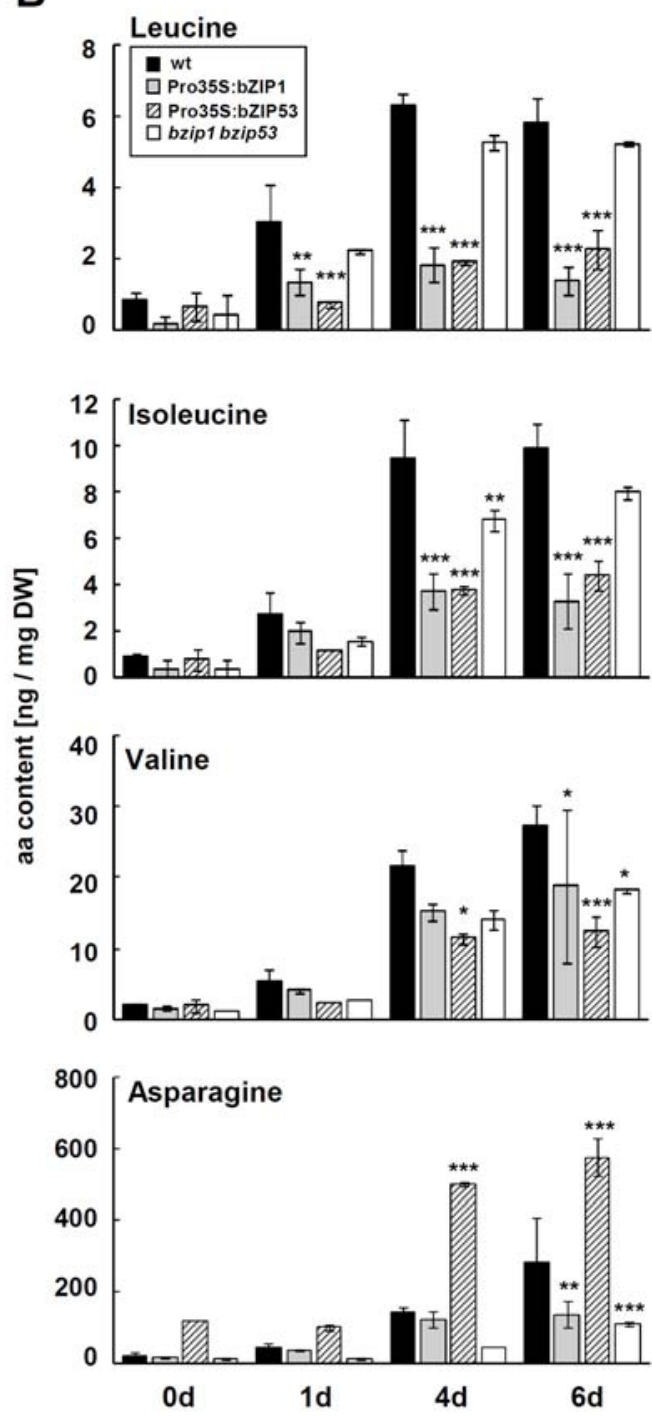

Figure 3. Quantitative analysis of the amount of aa in dark treated plants. Given are the levels of total aa content (A) Leu, Ile, Val and Asn (B). Aa levels of wt (black bars), Pro35S:bZIP1 (grey bar ars), Pro35S:bZIP53 (hatched bars) and bzip1 bzip53 (white bars) after 0,1, 4 and6 days of dark treatment are calculated as ng aa/mg dry weight (DW). Given are mean values of two independent experiments. Asterisks represent significant differences between wt, overexpressor and mutant plants at the indicated time point (two-way ANOVA, $\left.{ }^{*} \mathrm{p}<0.05 ; * * \mathrm{p}<0.01 ; * * * \mathrm{p}<0.001\right)$. 
bZIP53 up-regulated the activity of the ProDH promoter both after light and dark cultivation. In contrast to the results obtained in transgenic plants, expression of bZIP1 in protoplasts did not induce both reporter constructs indicating that additional factors are needed to fulfil its function in plants, which are not present in protoplasts. The differences observed in bZIP1 and bZIP53 function in protoplasts were not due to different protein levels as confirmed by immunoblot analysis (Supplemental Figure S7C online).

\section{bZIP1 and bZIP53 directly regulate ASN1 and ProDH promoter activity via G-boxes or ACT-cis elements in starvation response}

Using the protoplast transfection system, we analysed whether the starvation response is mediated by ACGT motifs, which represent typical binding sites for bZIP TFs (Jakoby et al., 2002). As summarized in Table S2, all promoters of the Asn biosynthesis genes and the promoters of ProDH and BCAT2 harbour at least one ACGT motif. In the ASN1 promoter two G-boxes (CACGTG) were found and the G-box 1 was identified as the crucial cis-element in mediating SnRK1- responses (Baena-Gonzalez et al., 2007). Sequential mutation in the ProASN1:GUS reporter gene demonstrated that the dark-induced transcription and the bZIP1/bZIP53-mediated enhancement of transcription depended exclusively on G-box 1 (Figure 5A). No alteration in the ProASN1:GUS reporter gene activity was observed with a loss-of-function mutation in G-box 2 indicating that the position of the hexameric CACGTG sequence within the promoter is important to mediate the starvation-related gene expression in protoplasts. In order to define whether the identified genes involved in aa metabolism are direct targets of the bZIP factors, Chromatin Immuno Precipitation (ChIP) experiments were performed with transgenic Arabidopsis lines expressing the HA-tagged version of bZIP1. By using primers which amplify the G- box $1 / 2$ promoter region, we could show direct binding of HA-bZIP1 proteins to the ASN1 promoter (Figure 5B). Previous results revealed pronounced differences in the regulation of ProDH and ASN1. The ProDH promoter harbours no G-box, but a C-box (GACGTC) and two ACT-elements (ACTCAT) proposed as bZIP binding sites involved in ProDH regulation (Satoh et al., 2004; Table S2). Whereas single mutations in the ACTCAT-element (Figure 5C) or C-boxes (Supplemental Figure S8 online) resulted in minor but significant effects on dark-induced ProDH activation, multiple mutations in two cis-elements completely abolished inducibility of the ProDH promoter. From these data we propose a crucial combinatorial in vivo function of these elements in the dark-induced ProDH activation. Recently published ChIP experiments demonstrated the in vivo binding of bZIP53 to the ProDH promoter (Weltmeier et al., 2006). In addition, ChIP analyses using primers surrounding the ACT elements and Pro35S:HA-bZIP1 plants also revealed a direct binding of bZIP1 to the ProDH promoter (Figure 5D). Immunoblot analysis of chromatin derived from light and dark grown plants showed equal amounts of HA-tagged bZIP1 protein in the ChIP assays (Figure 5E). Therefore, the binding activity of bZIP1 to the ProDH promoter was independent of the light/dark regime.

Multiple bZIP mutants and plants expressing EAR-repressor fusions of bZIP factors are partially impaired in darkinduced transcription of amino acid metabolic genes

The bzip1 bzip53 double mutant showed only limited impairment in dark-induced ProDH and ASN1 transcript accumulation (Figures 2C, 4C, Supplemental Figure S5 online). We therefore, applied an alternative loss-offunction approach. Fusions between bZIP53 and bZIP1 and the EAR repressor domain (Hiratsu et al., 2003) were generated and 
D

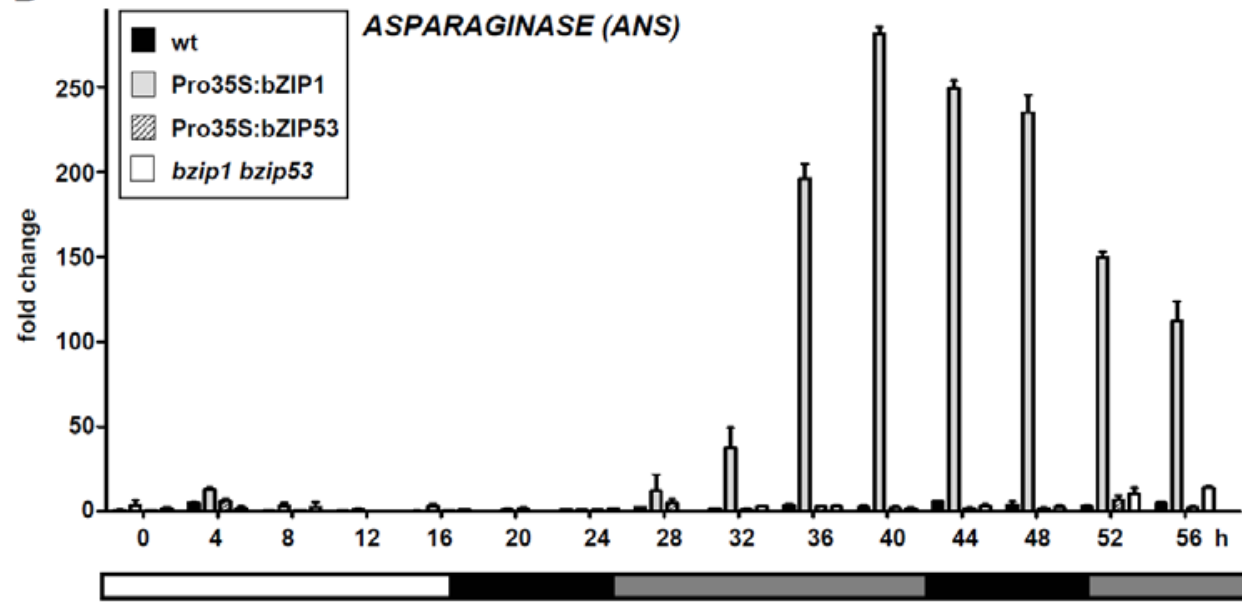

A

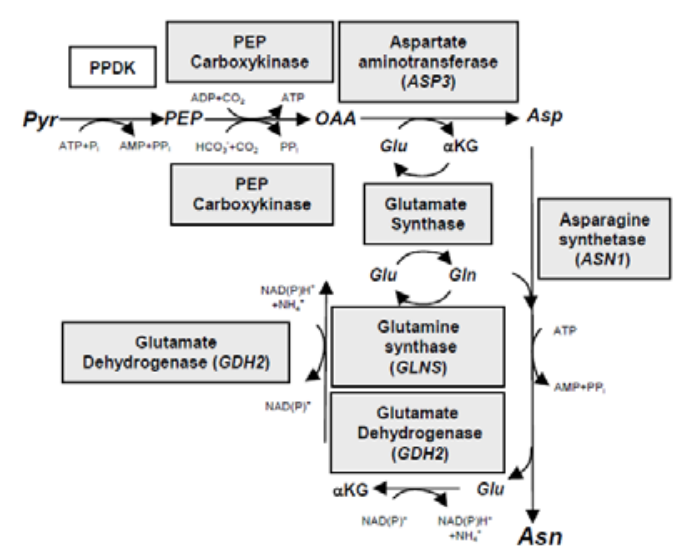

C

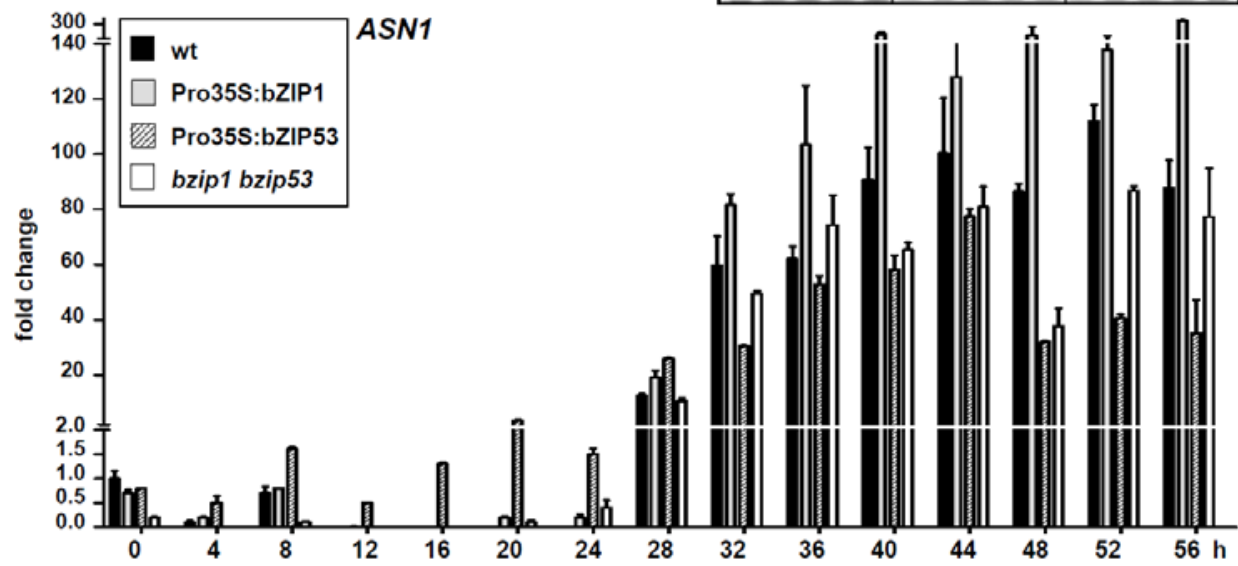

B

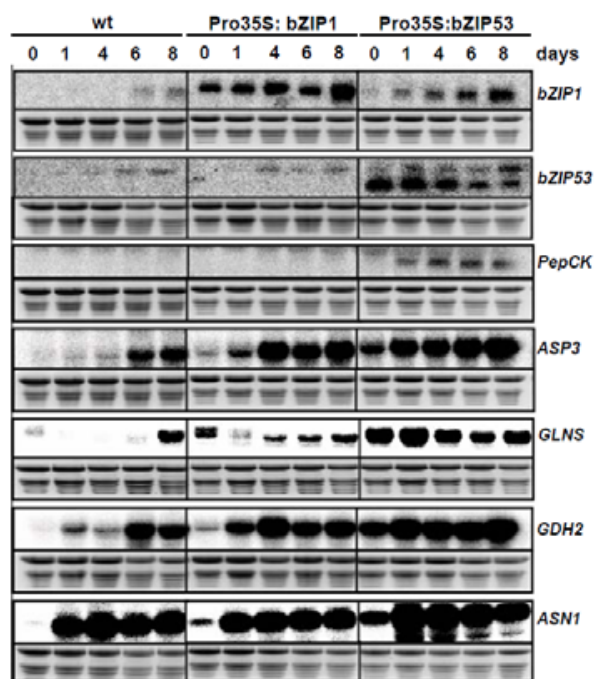

Figure 4. bZIP1 and bZIP53 control gene expression of Asn metabolism during extended night treatment. (A) The pathway of Asn biosynthesis according to Lin and Wu (2004). Pyr, pyruvate; PEP, phosphoenolpyruvate; $\alpha$ KG, $\alpha$-ketoglutarate; PPDK, pyruvate orthophosphate dikinase. (B) Northern analysis of the indicated genes corresponding to the enzymatic steps depicted in the Asn biosynthesis pathway in A after long-term dark treatment for $0-8 \mathrm{~d}$. Compared are wt, Pro35S:bZIP1, and Pro35S:bZIP53 plants. As a loading control, Ethidium bromide (EtBr) stainings are provided for each hybridisation experiment. Induction of ASN1 (C) or asparaginase gene (ANS) (D) after short-term dark treatment. wt (black bars), Pro35S:bZIP1 (grey bars), Pro35S:bZIP53 (hatched bars) and bzip1bzip53 (white bars) analysed by qPCR as described in Figure 1A. For visualizing the differences in transcript levels of ASN1, the y axes is broken twice at 2 fold induction. 
tested for their impact on the ProDH reporter in protoplasts. As shown in Figure $6 \mathrm{~A}$, the light- and dark-induced activation of the ProProDH:GUS reporter was completely abolished by EAR-bZIP1 and strongly reduced by EAR-bZIP53. Expression of the fusion proteins was confirmed by immunoblot analysis as demonstrated in Supplemental Figure S9A online. These data further substantiate our hypothesis that bZIP1 and bZIP53 play a crucial role in the control of dark-induced ProDH transcription. However, due to their heterodimerisation properties, other bZIPs presumably members of the C/S1 network are likely candidates for mediating the dark-induced starvation response (Ehlert et al., 2006). We therefore, included quadruple T-DNA mutants of bZIP1 and bZIP53 with different group C bZIPs (bzip1 bzip53 bzip9 bzip63 and bzip1 bzip53 bzip10 bzip25) in our study. As demonstrated in Supplemental Figure S9B,C online, depending on the particular bZIP gene, complete null alleles or "knockdown" alleles were obtained in the respective mutant lines. The accumulation of ASN1, ProDH (Figure 6B) and BCAT2 (Supplemental Figure S6D, online) transcripts was considerably impaired during extended dark treatment in the quadruple mutants, although no complete loss of transcript accumulation was observed. Surprisingly after long term dark treatment, gene expression was partially restored, indicating that the plant harbours regulatory mechanisms to substitute for the loss of particular bZIP proteins.

\section{DISCUSSION}

In this work, we have identified two bZIP TFs, namely bZIP1 and bZIP53, which translate low energy signals into an altered transcriptional pattern of aa metabolic genes in Arabidopsis. As outlined in the model in Figure 7, starvation activates in particular bZIP1 transcriptionally and posttranscriptionally - the latter by a conserved system of uORFs (Wiese et al., 2004; Weltmeier et al., 2009). Presumably by heterodimerisation with other members of the C/S1 bZIP TF network, bZIP1 and bZIP53 initiate the change in transcriptional activity by binding to ACGT or ACTCATlike cis-elements within the promoters of metabolic target genes. In conclusion, bZIP1 and bZIP53 are proposed to mediate transcriptional metabolic reprogramming in response to starvation.

The bZIP transcription factors bZIP1 and bZIP53 are regulated by energy deprivation, both on transcriptional and post-transcriptional level

In this work, bZIP1 - and to minor extends also bZIP53 - has been found to be transcriptionally up-regulated by conditions leading to energy deprivation. Feeding experiments with sucrose and glucose, but not 3-oMG represses bZIP1 and bZIP53 transcription (Figure 2E). 3-oMG is taken up by the cells but is not metabolised and appears not to signal via the hexokinasedependent sugarsignalling pathway (Cortes et al., 2003). These data indicate that sugarsignalling controls transcription of bZIP1 and bZIP53 supporting recent findings by Kang et al. (2010). Since long-time dark treatments, which frequently have been applied for dark-induced senescence studies (Gan, 2003; Lin and Wu, 2004; BuchananWollaston et al., 2005) do not reflect natural environmental conditions, shortterm experiments have been performed which describe detailed expression changes after extended night treatments and defined bZIP1 and bZIP53 as putative transcripttional regulators in the starvation response (Figure 1A). For assaying starvation responses, plant and protoplast systems have been applied using culture conditions in the dark or incubation with the photosystem II inhibitor DCMU 
A

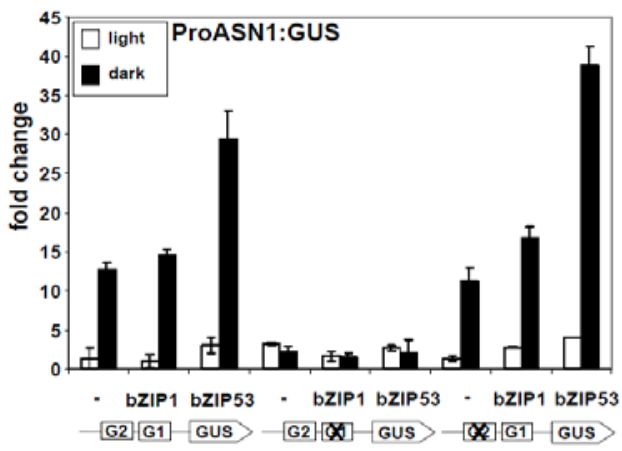

B

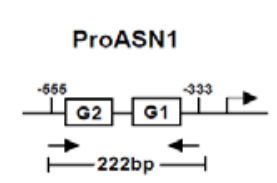

C

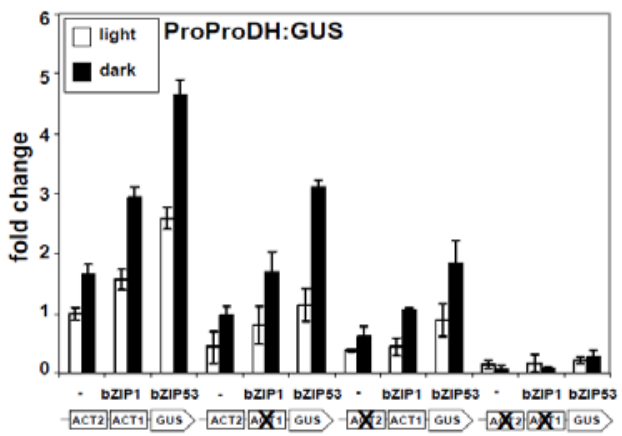

D

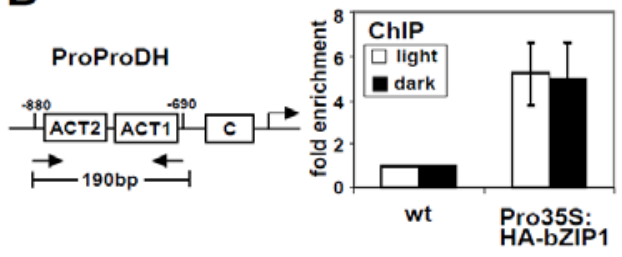

$\mathbf{E}$

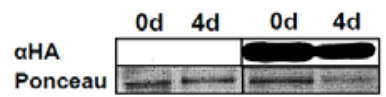

Figure 5. bZIP1 binds directly to the ASN1 and ProDH promoters and mediates starvation responses via G-box (CACGTG) or ACTCAT cis-elements. (A) Arabidopsis protoplasts have been transformed with a ProASN1:GUS reporter construct or the indicated promoter mutations. After co-transformation with the effector plasmids (Pro35S:bZIP1 or Pro35S:bZIP53), reporter induction was compaired in constant darkness (black bars) or in constant light (white bars) conditions. Given is the fold change with respect to the empty vector control experiment without any bZIP construct added (-) under constant light. (B) Direct binding of bZIP1 to the ASN1 promoter has been demonstrated by ChIP. ASN1 promoter structure and primer binding sites are indicated on the left. Chromatin extracts from wt plants and Pro35S:HA-AtbZIP1 are subjected to qPCR analysis with ASN1 promoter specific primers after immunoprecipitation with an anti-HA antibody $(\alpha-\mathrm{HA}) . \mathrm{Ct}$ values for Pro35S:HA-bZIP1 samples are subtracted from the $\mathrm{Ct}$ values of the equivalent wt. For normalization an actin (ACT7) gene has been used.
Calculated are induction levels with respect to the wt samples. Given are mean values and standard deviation of 2 independent experiments. (C) Analysis of the ProProDH:GUS construct as described in A. Compared are the non-mutated promoter and ACTCAT (ACT) specific mutations. (D) ChIP experiment of wt and Pro35S:HAbZIP1 plants using $\alpha \mathrm{HA}$ antibody and chromatin isolated from 3-week old plants grown under normal light/dark cycle (white bars) or plants cultivated in an extended night for 4 days (black bars). Error bars represent mean values and standard deviation of 3 repetitions. ProDH promoter structure and primer binding sites are indicated on the left. (E) Immuno blot analysis of chromatin derived from wt and Pro35S:HA-bZIP1 plants detected with an $\alpha \mathrm{HA}$ antibody indicates a comparable HA-bZIP1 protein abundance in light and dark treated plants. As a loading control, Ponceau staining of the protein preparation is given (lower panel).

(e.g. Figure 1A, Supplemental Figure S7A,B online). Recently, other stresses such as anaerobic conditions have been found equally useful (Baena-Gonzalez et al., 2007). Altogether these treatments let to comparable responses with respect to transcription of starvation-induced genes. Environmental stresses which also lead to a low energy status of the cell can be assumed to interfere with the primary metabolism and therefore might also input into the starvation signalling network (Baena-Gonzalez and Sheen, 2008). Recent bioinformatic network analyses point into the direction that bZIP1 is regulated by the circadian clock (Gutierrez et al., 2008). Interestingly, CIRCADIAN CLOCK ASSOCIATED 1 (CCA1) which is an integral component of the Arabidopsis clock (Yakir et al., 2007) was shown to directly bind to the bZIP1 promoter (Gutierrez et al., 2008). Although a detailed molecular analysis is still elusive, it is tempting to speculate that a regulatory energy management network exists in plant cells, which integrates $\mathrm{C}$ and $\mathrm{N}$ availability and clock-initiated day/night rhythms to control bZIP1-dependent gene expression. In addition to transcriptional regulation, further post-transcriptional mechanisms might account for bZIP1 and bZIP53 regulation. For all group S1 bZIPs a posttranscriptional repression by sucrose 
A

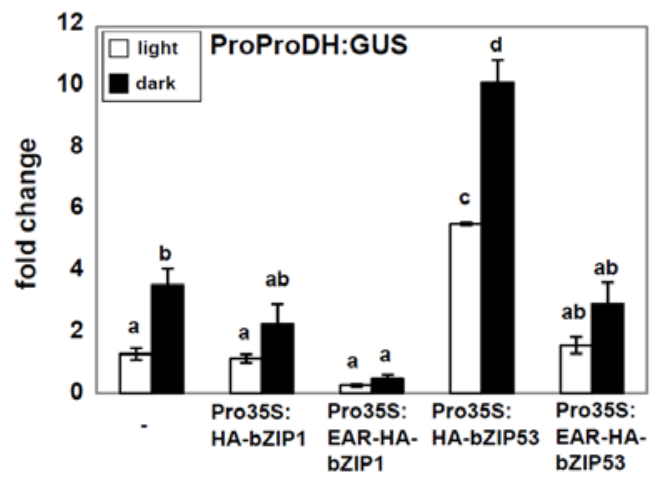

B
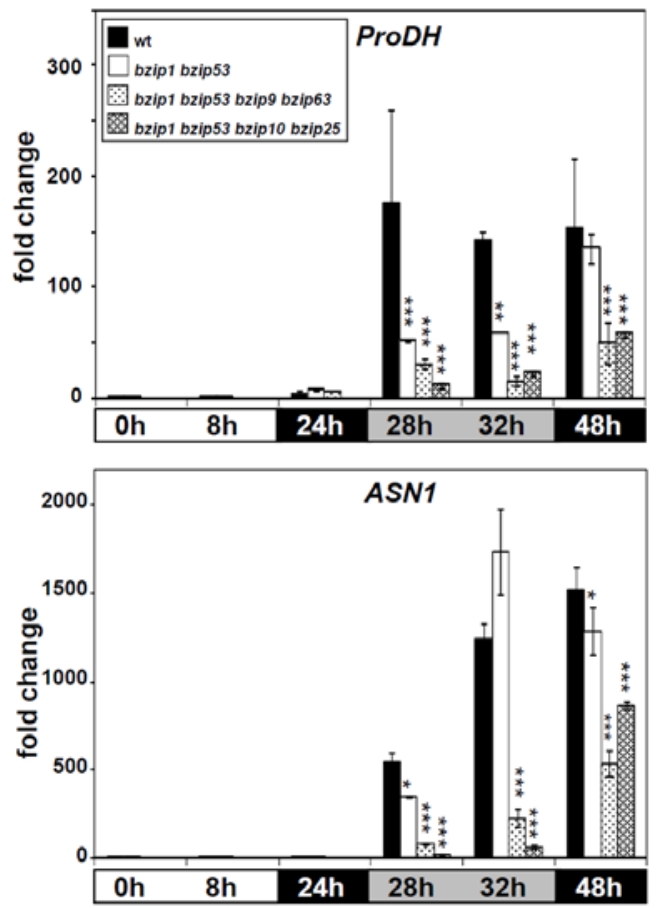

Figure 6. Impact of bZIP factors on target gene expression using bZIP specific loss-of-function approaches. (A) EAR repressor fusions of bZIP1 and bZIP53 reveal a regulatory function in regulation of darkinduced ProDH transcription. Arabidopsis protoplasts have been transiently transformed with a ProProDH:GUS reporter and co-transfected with Pro35S driven reporters (HA-bZIP1, HA-bZIP53, HA-EAR-bZIP1, HA-EARbZIP53). Induction by cultivation in constant dark (black bars) conditions is compared to expression in constant light (white bars). Depicted is the fold change compared to the promoter in the light. Significance was tested by One-way ANOVA analysis following Tukey`s post-test, $\mathrm{p}<0,05$. Expression of the effector constructs has been confirmed by immuno blot analysis (Supplemental Figure S9A online). (B) qPCR analysis of ProDH and ASN1 after extended night treatment as described in Figure 1. Given are fold change values comparing wt expression at the time point " 0 ". Asterisks represent significant differences between wt and mutant plants at the indicated time point (two-way ANOVA, $* \mathrm{p}<0,05 ; * * \mathrm{p}<0,01 ; * * * \mathrm{p}<0,001)$. Impaired expression of the corresponding bZIP genes is demonstrated in Supplemental Figure S9B, C online.
(SIRT) was demonstrated (Weltmeier et al., 2009). With respect to bZIP1 and bZIP53, these mechanisms result in an additive effect leading to high intracellular levels of the bZIP TFs during energy deprivation (Figure 7). Thus, bZIP1 and bZIP53 are candidates to execute a crucial function in metabolic reprogramming during the starvation response.

\section{bZIP1 is regulated post-translationally by a starvation-derived signal}

Although bZIP1 is strongly up-regulated by transcriptional and posttranscriptional mechanisms, its function in gene regulation depends on an additional signal which is initiated by dark-induced energy deprivation. Importantly, this observation obtained by studying transgenic plants was not detectable in the protoplast system by us and others (Baena-Gonzalez et al., 2007). Obviously, an unknown component is missing in the protoplast system and therefore, these findings clearly emphasize the importance of studying the whole plant system. bZIP53 is predominantly regulated on the level of protein amount. When overexpressed, it activates transcription independent of the energy status of the cell whereas bZIP1 activity is further fine-tuned on post-translational level by a starvationdependent signal. Recently, KIN10 and KIN11, two kinases of the SnRK1 family have been demonstrated to orchestrate starvation responses, in particular on transcriptional level (Baena-Gonzalez et al., 2007; Baena-Gonzalez and Sheen, 2008). It remains to be analysed whether the starvation signal controlling bZIP1 function is directly or indirectly mediated by these kinases.

\section{bZIP1 and bZIP53 show overlapping but distinct functions in the low energy response}


In this work, ectopic overexpression of bZIP1 results in an early senescence phenotype in the dark, characterized by rapid loss of chlorophyll (Figure 1C,D). As defined by marker gene expression, this phenotype reflects a dark-induced starvation response but not the natural senescence (Supplemental Figure S3 online; van der Graaff et al., 2006). This early senescence phenotype is less

pronounced in Pro35S:bZIP53 plants. Furthermore, distinct differences in the function of bZIP1 and bZIP53 become obvious when these plants were studied under normal day/night cycle. As confirmed by immunoblot analysis, highlevel expression of bZIP1 did not lead to obvious phenotypic changes whereas medium level-expression of bZIP53 results in significantly reduced plant growth (Alonso et al., 2009). These findings clearly indicate pronounced differences in bZIP1 and bZIP53 function. Overexpression of bZIP1 and bZIP53 in transgenic plants revealed that both TFs are capable to regulate aa metabolic genes proposed to be up-regulated during dark-induced starvation response such as ProDH, ASN1, Asn biosynthesis and BCAA metabolism (e.g. Figure 2C, 4B,C, Supplemental Figure S6C online). ASN1, the key gene of Asn biosynthesis pathway is characterised by a complex transcriptional regulation (Lam et al., 1998; Lam et al., 2003). Transcription of Asn pathway genes such as ASP3, GDH2 and ASN1 appears to be coregulated and is induced after dark treatment but repressed when sugar is available (Lin and $\mathrm{Wu}, 2004$ ). In light, ectopic expression of bZIP53 leads to constitutive activation of ProDH, ASP3, GDH2 and ASN1, whereas bZIP1 provokes only minor effects. Changes in the transcriptional levels of aa metabolic genes are well reflected on the level of aa. For instance, the amount of proline is significantly reduced in Pro35S:bZIP1 and
Pro35S:bZIP53 plants (Figure 2D). Contrarily, the amount of Asn is induced exclusively in Pro35S:bZIP53 plants (Figure 3B). As demonstrated on transcriptional level, bZIP1 but not bZIP53 enhances expression of an ASPARAGINASE gene (Figure 4D) which leads to the degradation of Asn (Bruneau et al., 2006). Again, bZIP53 and bZIP1 show distinct differences in target gene selection. This might explain the observed differences in the metabolite profiles. Ectopic expression of bZIP1 and bZIP53 leads to reduced levels of BCAA (Figure 3B), indicating the involvement of both TFs in a BCAA degradation pathway. BCAT2 transcription follows the pattern of the other analysed, dark-induced aa metabolic genes, and bZIP1 and bZIP53 strongly enhance BCAT2 transcription (Supplemental Figure S6C online). Yet, it is not clear whether the BCAT2 enzyme is involved in an anabolic or catabolic context. In the dark, chloroplastic proteins such as RUBISCO are degraded to provide aa to the starved nitrogen metabolism. It was speculated that the deamination reaction of BCAT2 in the dark supplies the cell with Glu which is essential for Asn biosynthesis (Schuster and Binder, 2005). Hence, the dark induced BCAT2 expression as well as the BCAA degradation might be closely linked to the dark-induced Asn biosynthesis and consequently, appear to be regulated in a coordinated fashion by the same set of bZIP TFs. Differences in function have already been described for bZIP53 and bZIP1 (Weltmeier et al., 2009). E. g., heterodimers enclosing bZIP53 control the expression of seed maturation genes involved in desiccation tolerance, storage compound synthesis and source sink control, such as ASN1 (Alonso et al., 2009). Although bZIP1 heterodimers share the capacity to activate seed maturation genes in protoplasts, bZIP1 appears not to be involved in regulation of these genes during 
seed maturation (Weltmeier et al., 2009). Altogether, bZIP53 and bZIP1 have partially overlapping but distinct functions which are probably defined by expression pattern and/ or post- translational mechanisms.

Transcriptional control of amino acid metabolic genes by bZIP1 and bZIP53 is mediated by binding G-box, C-box or ACTCAT cis-elements

As demonstrated in transiently transfected protoplasts, one of the two G-boxes (G-box 1) in the promoter of ASN1 is essential for the dark-activation of this gene (Figure 5A). Our promoter deletion experiments further substantiate the hypothesis that bZIP1 and bZIP53 signal via the G-box 1 in the ASN1 promoter. G-box-like cis-elements, which are characterised by their ACGT core, are typical binding sites for bZIP TFs as demonstrated for bZIP53 by in vitro and in vivo binding assays (Alonso et al., 2009). We used HA-tagged bZIP1 in ChIP experiments to confirm its direct binding to ProDH and ASN1 promoter regions (Figure 5B,D). Although, due to overexpression, the ChIP data have to be interpreted with care, they are supported by results of the protoplast assays. Our combined data strongly suggest that the analysed ProDH and ASN1 promoters are direct in vivo targets of bZIP1. Dark-induced recruitment of bZIP1 to its target promoters is one possible regulatory mechanism which would explain stimulus induced target gene activation. However, dark induced enhancement of bZIP1 DNA-binding to the ProDH promoter was not detected in the ChIP experiments, at least at the time points used in this study. Surprisingly enough, the ProDH promoter harbours no G-box but a closely related, ACGT core-containing Cbox and two ACTCAT motifs. The latter was shown to be bound by group S1 bZIPs and is involved in hypoosmolarity response
(Satoh et al., 2002; Satoh et al., 2004; Weltmeier et al., 2006). Multiple mutations in ACTCAT and C-box elements confirm a crucial and additive impact of all these ciselements on basic and inducible ProDH promoter activity. These data suggest that differences in ASN1 and ProDH expression patterns are caused by their promoter structure defined by combination and location of the identified cis-elements. Furthermore, bZIP heterodimerisation might alter target site recognition and therefore, it is tempting to speculate that different promoters will recruit particular sets of bZIP heterodimers.

\section{Redundant bZIP factors can partially substitute for loss of bZIP1 and bZIP53}

Expression of bZIP1 and bZIP53 fusion proteins containing a C-terminal EAR repressor domain (Hiratsu et al., 2003) completely abolishes or significantly reduces dark-induced ProDH expression in protoplasts (Figure 6A). Since the repressor-modified TF blocks specific promoter binding sites when overexpressed, this method was applied to compete with redundantly active TFs to interfere with their function. As single bzip1 and bzip53 T-DNA mutants and even a bzip1 bzip53 double mutant do not lead to dramatic impairment of aa target gene expression (Supplemental Figure S5 online), the protoplast data support the view that a functional redundant bZIP transcription factor network is operating in the starvation response. bZIP2 expression patterns are slightly similar to the one observed for bZIP53. However, since bZIP2 T-DNA insertion lines are not available and bZIP2 overexpression results in a severely dwarfed, sterile plants bZIP2 could not be included in the experimental set-up. Strong interference with normal plant growth was also observed by overexpression of bZIP11 (Hanson et al., 2008). Inducible expression 
combined with transcriptome analysis was used to identify ProDH and ASN1 as bZIP11-controlled genes. However, although bZIP11 has the capacity to regulate these metabolic genes, it is probably not involved in the starvation response as its expression is upregulated by sugar and down-regulated by darkness.

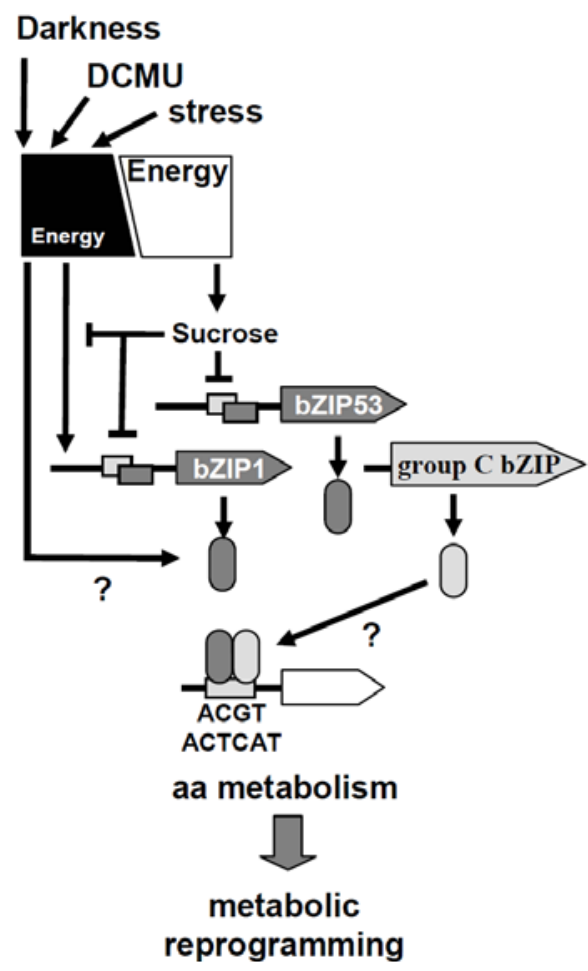

Figure 7. Model summarizing the function of bZIP factors in energy deprivation response. For details see text.

As depicted in Supplemental Figure S1 online, several bZIP genes such as bZIP41 or bZIP54 show appropriate expression patterns to fulfil a function in energy deprivation response. Also the group $\mathrm{C}$ factors bZIP63, bZIP9 and bZIP25 which form heterodimers with group S1 might be candidates. Specific bZIP heterodimerisation has been shown to be important for bZIP53 activity and function (Ehlert et al., 2006; Weltmeier et al., 2006). Consequently, quadruple C/S1 T-DNA insertion mutants show strongly impaired dark-induced target gene expression (Figure 6B, Supplemental Figure S9D online).
Surprisingly, this effect is only transient and the plant can partially compensate for the loss of bZIP gene activity during longterm dark adaptation. Overlapping functional redundancy appears to be a frequently observed feature in particular in signalling networks. The regulation of target genes by $\mathrm{C} / \mathrm{S} 1 \mathrm{bZIPs}$ is complex, but however more stable with respect to mutations and more flexible in terms of its potential to fine tune regulation. Although the plant obviously benefits from this flexibility, the regulatory circuits controlling this TF network remain elusive. Knowledge on the transcriptional regulators is crucial for understanding the plant energy control system and a first step to establish biotech approaches to increase yield and stress tolerance of crop plants.

\section{MATERIALS AND METHODS}

For plant and protoplast transformation, aa measurement, qPCR, Northern and ChIP experiments (see Supplemental Methods), Arabidopsis thaliana ecotype Columbia (Col-0) was grown on soil under long day conditions of $16 \mathrm{~h} \mathrm{light} / 8 \mathrm{~h}$ dark cycles. Dark treatment was performed from 4 h up to 8 days using 3 week old soil grown plants. For Northern Analysis the first harvesting time point (0d) was at $5 \mathrm{pm}$ in the middle of the light period. Material for qPCR was harvested as indicated, starting at the beginning of the light period (8:00 am). For hydroponic culture, the procedure described in Gibeaut et al. (1997) has been modified. 3- week-old plants were grown on a mesh support under short-day conditions. The media were supplemented with sugars according to the description in Figure 2E. Floral dip transformations have been performed by using the Agrobacterium tumefaciens strain GVG3101 (Weigel and Glazebrook, 2002). Transgenic plants and T-DNA insertion lines are summarized in Table S3. Homozygous mutants were 
identified by PCR as described in (http://signal.salk.edu/tdnaprimers.2.html) using the primers described in Table S4. Statistical analysis was performed with the GraphPad Prism software using the tests indicated in the figure legend.

\section{Accession Numbers}

Arabidopsis Genome Initiative identifiers for the genes mentioned in this article are as follows: bZIP53 (At3g62420), bZIP1 (At5g49450), bZIP63 (At5g28770), bZIP10 (At4g02640), bZIP25 (At3g54620), bZIP9 (At5g24800), ProDH (At3g30775), ASN1 (At3g47340), GDH2 (At5g07440), ASP3 (At5g11520), GLNS (At5g37600), PepCK (At5g65690), ANS (At3g16150), CAB (At1g29920), SAG103 (At1g10140), YLS3 (At2g44290), BCAT2 (At1g10070), LEA76 (At3g15670), UBI5 (At3g62250), ACT7 (At5g09810).

\section{ACKNOWLEDGMENTS}

We are grateful to Jennifer Krüger, Anna Herman and Bettina Stadelhofer for their valuable technical assistance. WD-L is supported by a grant of the Deutsche Forschungsgemeinschaft (DFG).

\section{REFERENCES}

Alonso, R., Oñate-Sánchez, L., F., W., Ehlert, A., Diaz, I., Dietrich, K., Vicente-Carbajosa, J., and Dröge-Laser, W. (2009) A pivotal role of the basic leucine zipper transcription factor AtbZIP53 in the regulation of seed maturation gene expression based on heterodimerisation and protein complex formation. Plant Cell 21, 1747-1761.

Baena-Gonzalez, E., and Sheen, J. (2008)

Convergent energy and stress signaling. Trends Plant Sci. 13: 474-482.

Baena-Gonzalez, E., Rolland, F., Thevelein, J.M. , and Sheen, J. (2007) A central integrator of transcription networks in plant stress and energy signalling. Nature 448: 938-942.
Bruneau, L., Chapman, R., and Marsolais, F.

(2006) Co-occurrence of both L-asparaginase subtypes in Arabidopsis: At3g16150 encodes a $\mathrm{K}+$-dependent L-asparaginase. Planta 224: 668679.

Buchanan-Wollaston, V., Page, T., Harrison, E., Breeze, E., Lim, P.O., Nam, H.G., Lin, J.F., Wu, S.H., Swidzinski, J., Ishizaki, K., and Leaver, C.J. (2005) Comparative transcriptome analysis reveals significant differences in gene expression and signalling pathways between developmental and dark/starvation-induced senescence in Arabidopsis. Plant J. 42: 567-585.

Cortes, S., Gromova, M., Evrard, A., Roby, C., Heyraud, A., Rolin, D.B., Raymond, P., and Brouquisse, R.M. (2003) In plants, 3-omethylglucose is phosphorylated by hexokinase but not perceived as a sugar. Plant Physiol. 131: 824-837.

Diebold, R., Schuster, J., Daschner, K., and Binder, S. (2002) The branched-chain amino acid transaminase gene family in Arabidopsis encodes plastid and mitochondrial proteins. Plant Physiol. 129: 540-550.

Ehlert, A., Weltmeier, F., Wang, X.,

Mayer, C.S., Smeekens, S., VicenteCarbajosa, J., and Dröge-Laser, W. (2006) Two-hybrid protein-protein interaction analysis in Arabidopsis protoplasts: Establishment of a heterodimerisation map of group-C and $\mathrm{S}$ bZIP transcription factors. Plant J. 46: 890 - 900.

Fode, B., and Gatz, C. (2009). Chromatin immunoprecipitation experiments to investigate in vivo binding of Arabidopsis transcription factors to target sequences. Methods Mol Biol. 479, 261-272.

Gan, S. (2003). Mitotic and postmitotic senescence in plants. Sci Aging Knowledge Environ 2003, re7.

Gibeaut, D.M., Hulett, J., Cramer, G.R., and Seemann, J.R. (1997). Maximal biomass of Arabidopsis thaliana using a simple, lowmaintenance hydroponic method and favorable environmental conditions. Plant Physiol. 115: 317-319.

Gutierrez, R.A., Stokes, T.L., Thum, K., Xu, X., Obertello, M., Katari, M.S., Tanurdzic, M., Dean, A., Nero, D.C., McClung, C.R., and Coruzzi, G.M. (2008) Systems approach identifies an organic nitrogen-responsive gene network that is regulated by the master clock control gene CCA1. Proc. Natl. Acad. Sci. 105: 4939-4944. 
Hanson, J., Hanssen, M., Wiese, A., Hendriks, M.M.W.B., and S, S. (2008) The sucrose regulated transcription factor bZIP11 affects amino acid metabolism by regulating the expression of Asparagin Synthase1 and Proline Dehydrogenase2. Plant J. 53: 935-949.

Hellmann, H., Funck, D., Rentsch, D., and

Frommer, W.B. (2000) Hypersensitivity of an Arabidopsis sugar signaling mutant toward exogenous proline application. Plant Physiol. 122: $357-368$.

Hiratsu, K., Matsui, K., Koyama, T., and Ohme-

Takagi, M. (2003) Dominant repression of target genes by chimeric repressors that include the EAR motif, a repression domain, in Arabidopsis. Plant J. 34: 733-739.

Jakoby, M., Weisshaar, B., Dröge-Laser, W., Vicente-Carbajosa, J., Tiedemann, J., Kroj, T., and Parcy, F. (2002) bZIP transcription factors in Arabidopsis. Trends Plant Sci. 7: 106111.

Kang, S.G., Price, J., Lin, P.C., Hong, J.C., and Jang, J.C. (2010). The Arabidopsis bZIP1 Transcription Factor Is Involved in Sugar Signaling, Protein Networking, and DNA Binding. Mol Plant. doi:10.1093/mp/ssp115

Kirby, J., and Kavanagh, T.A. (2002). NAN fusions: a synthetic sialidase reporter gene as a sensitive and versatile partner for GUS. Plant J. $32,391-400$.

Lam, H.M., Peng, S.S., and Coruzzi, G.M. (1994).

Metabolic regulation of the gene encoding glutamine-dependent asparagine synthetase in Arabidopsis thaliana. Plant Physiol. 106: 13471357.

Lam, H.M., Hsieh, M.H., and Coruzzi, G. (1998).

Reciprocal regulation of distinct asparagine synthetase genes by light and metabolites in Arabidopsis thaliana. Plant J. 16: 345-353.

Lam, H.M., Wong, P., Chan, H.K., Yam, K.M., Chen, L., Chow, C.M., and Coruzzi, G.M. (2003). Overexpression of the ASN1 gene enhances nitrogen status in seeds of Arabidopsis. Plant Physiol. 132, 926-935.

Lin, J.F., and Wu, S.H. (2004). Molecular events in senescing Arabidopsis leaves. Plant J. 39: 612628.

Nakashima, K., Satoh, R., Kiyosue, T., Yamaguchi-Shinozaki, K., and Shinozaki, K. (1998). A gene encoding proline dehydrogenase is not only induced by proline and hypoosmolarity, but is also developmentally regulated in the reproductive organs of Arabidopsis. Plant Physiol 118: 1233-1241.

Pilot, G., Stransky, H., Bushey, D.F., Pratelli, R., Ludewig, U., Wingate, V.P., and Frommer, W.B. (2004). Overexpression of GLUTAMINE DUMPER1 leads to hypersecretion of glutamine from Hydathodes of Arabidopsis leaves. Plant Cell 16, 1827-1840.

Polge, C., and Thomas, M. (2007).

SNF1/AMPK/SnRK1 kinases, global regulators at the heart of energy control? Trends Plant Sci. 12: $20-28$.

Satoh, R., Nakashima, K., Seki, M., Shinozaki, K., and Yamaguchi-Shinozaki, K. (2002). ACTCAT, a novel cis-acting element for proline- and hypoosmolarity-responsive expression of the ProDH gene encoding proline dehydrogenase in Arabidopsis. Plant Physiol. 130: 709-719.

Satoh, R., Fujita, Y., Nakashima, K., Shinozaki, K., and Yamaguchi-Shinozaki, K. (2004). A novel subgroup of bZIP proteins functions as transcriptional activators in hypoosmolarityresponsive expression of the ProDH gene in Arabidopsis. Plant Cell Physiol. 45: 309-317.

Schuster, J., and Binder, S. (2005). The

mitochondrial branched-chain aminotransferase (AtBCAT-1) is capable to initiate degradation of leucine, isoleucine and valine in almost all tissues in Arabidopsis thaliana. Plant Mol Biol. 57: 241-254.

Sprenger-Haussels, M., and Weisshaar, B. (2000). Transactivation properties of parsley prolinerich bZIP transcription factors. Plant J. 22, 1-8.

Usadel, B., Blasing, O.E., Gibon, Y., Retzlaff, K., Hohne, M., Gunther, M., and Stitt, M. (2008). Global transcript levels respond to small changes of the carbon status during progressive exhaustion of carbohydrates in Arabidopsis rosettes. Plant Physiol. 146: 1834-1861.

van der Graaff, E., Schwacke, R., Schneider, A., Desimone, M., Flugge, U.I., and Kunze, R. (2006). Transcription analysis of arabidopsis membrane transporters and hormone pathways during developmental and induced leaf senescence. Plant Physiol. 141: 776-792.

Weigel, R., and Glazebrook, J. (2002). Arabidopsis: A Laboratory Manual. (New York: Cold Spring Harbour Laboratory Press).

Weltmeier, F., Ehlert, A., Mayer, C.S., Dietrich, K., Wang, X., Schütze, K., Harter, K., Vicente-Carbajosa, J., and Dröge-Laser, W. (2006). Combinatorial control of Arabidopsis 
proline dehydrogenase transcription by specific heterodimerisation bZIP transcription factors. EMBO J. 25: 3133 - 3143.

Weltmeier, F., Rahmani, F., Ehlert, A., Dietrich, K., Schütze, K., Wang, X., Chaban, C., Hanson, J., Teige, M., Harter, K., VicenteCarbajosa, J., Smeekens, S., and DrögeLaser, W. (2009). Expression patterns within the Arabidopsis C/S1 bZIP transcription factor network: availability of heterodimerization partners controls gene expression during stress response and development. Plant Mol Biol. 69: 107-119.

Wiese, A., Elzinga, N., Wobbes, B., and Smeekens, S. (2004). A conserved upstream open reading frame mediates sucrose-induced repression of translation. Plant Cell. 16: 1717-1729.

Wiese, A., Elzinga, N., Wobbes, B., and Smeekens, S. (2005). Sucroseinduced translational repression of plant bZIP-type transcription factors. Biochem. Soc. Trans. 33, 272-275.

Yakir, E., Hilman, D., Hassidim, M., and Green,

R.M. (2007). CIRCADIAN CLOCK

ASSOCIATED1 transcript stability and the entrainment of the circadian clock in Arabidopsis. Plant Physiol. 145: 925-932. 
 expression in seed maturation
}

\section{A Pivotal Role of the Basic Leucine Zipper Transcription Factor bZIP53 in the Regulation of Arabidopsis Seed Maturation Gene Expression Based on Heterodimerization and Protein Complex Formation ${ }^{\mathrm{W}}$}

\author{
Rosario Alonso, ${ }^{a}$ Luis Oñate-Sánchez, ${ }^{a}$ Fridtjof Weltmeier, ${ }^{\mathrm{b}}$ Andrea Ehlert, ${ }^{\mathrm{b}}$ Isabel Diaz, ${ }^{\mathrm{a}}$ Katrin Dietrich, ${ }^{\mathrm{b}}$ \\ Jesús Vicente-Carbajosa, ${ }^{\mathrm{a}, 1}$ and Wolfgang Dröge-Laser ${ }^{\mathrm{b}, 1,2}$ \\ ${ }^{a}$ Centro de Biotecnología y Genómica de Plantas Universidad Politécnica de Madrid, Escuela Técnica Superior Ingenieros \\ Agrónomos, 28223 Pozuelo de Alarcón, Madrid, Spain \\ ${ }^{\mathrm{b}}$ Albrecht-von-Haller Institut, Universität Göttingen, D-37073 Göttingen, Germany
}

Transcription of Arabidopsis thaliana seed maturation (MAT) genes is controlled by members of several transcription factor families, such as basic leucine zippers (bZIPs), B3s, MYBs, and DOFs. In this work, we identify Arabidopsis bZIP53 as a novel transcriptional regulator of MAT genes. bZIP53 expression in developing seeds precedes and overlaps that of its target genes. Gain- and loss-of-function approaches indicate a correlation between the amount of bZIP53 protein and MAT gene expression. Specific in vivo and in vitro binding of bZIP53 protein to a G-box element in the albumin $2 S 2$ promoter is demonstrated. Importantly, heterodimerization with bZIP10 or bZIP25, previously described bZIP regulators of MAT gene expression, significantly enhances DNA binding activity and produces a synergistic increase in target gene activation. Fulllevel target gene activation is strongly correlated with the ratio of the correspondent bZIP heterodimerization partners. Whereas bZIP53 does not interact with ABI3, a crucial transcriptional regulator in Arabidopsis seeds, ternary complex formation between the bZIP heterodimers and ABI3 increases the expression of MAT genes in planta. We therefore propose that heterodimers containing bZIP53 participate in enhanceosome formation to produce a dramatic increase in MAT gene transcription.

\section{INTRODUCTION}

As an outstanding adaptation of terrestrial plants, seed formation favors dispersal of species and allows the interruption of the life cycle and its resumption once optimal growth conditions are newly established (for review, see Vicente-Carbajosa and Carbonero, 2005; Weber et al., 2005; Santos-Mendoza et al., 2008). Seeds are formed after a double fertilization event, triggering the development of a complex organ, which comprises the embryo, the endosperm, and the seed coat derived from the integuments and other surrounding layers of maternal origin. Seed development can be divided into three phases: first, embryogenesis is characterized by cell division and differentiation until embryo morphology is established. Second, the maturation phase is dominated by storage compound accumulation, growth arrest, and acquisition of desiccation tolerance. Third, the embryo can enter into a dormancy state that is broken upon germination. With respect to seed morphology, physiology, and gene regulation, considerable variations occur among species. Arabidopsis

${ }^{1}$ These authors contributed equally to this work.

${ }^{2}$ Address correspondence to wdroege@gwdg.de.

The author responsible for distribution of materials integral to the findings presented in this article in accordance with the policy described in the Instructions for Authors (www.plantcell.org) is: Wolfgang DrögeLaser (wdroege@gwdg.de).

"Online version contains Web-only data.

www.plantcell.org/cgi/doi/10.1105/tpc.108.062968 thaliana has been developed as a well-established model system for dicot seed development, and several similarities and differences with monocot model systems have been described (Vicente-Carbajosa and Carbonero, 2005; Santos-Mendoza et al., 2008).

Important programs of gene expression related to the metabolic changes that occur during seed maturation are highly coordinated and tightly regulated (Gutierrez et al., 2007). An understanding of gene expression control in the seed was tackled from early studies in plant molecular biology, with maize (Zea mays) Opaque2 (O2) representing a hallmark as one of the first plant transcription factor (TF) genes cloned and characterized (Hartings et al., 1989; Schmidt et al., 1990). Similarly, orthologous genes from wheat (Triticum aestivum) (SPA) and barley (Hordeum vulgare) (BLZ2) play the same roles as $\mathrm{O} 2$ in their corresponding species (Albani et al., 1997; Oñate et al., 1999). In dicot species, key TFs have been characterized that control gene expression programs during seed maturation.

The class of maturation genes (MAT) expressed during seed maturation typically includes seed storage protein (SSP) genes, such as albumin and cruciferin genes, which are induced in early or mid-maturation phase. The late embryogenesis abundant (LEA) genes are induced at later stages of maturation and include genes proposed to be involved in acquisition of desiccation tolerance (for review, see Tunnacliffe and Wise, 2007). MAT promoter analyses have revealed several conserved 
cis-regulatory elements with functional relevance in the control of gene expression during seed maturation. Among them, G-boxrelated ACGT elements, RY (CATGCA), AACA, and CTTT motifs are the best described examples (for review, see VicenteCarbajosa and Carbonero, 2005). The corresponding associated TFs belong to the basic leucine zipper (bZIP), B3, MYB, and DOF TF families, respectively. Cooperation of these regulatory units in the control of gene expression appears to be an evolutionarily conserved pattern that can be traced back to the origins of the Spermaphyta (Vicente-Carbajosa and Carbonero, 2005; Schallau et al., 2008).

TFs of the bZIP class, related to cereal O2-type TFs, have been identified in Arabidopsis (Lara et al., 2003), namely, bZIP10 and bZIP25, which have been classified into group $\mathrm{C}$ of the Arabidopsis bZIP TF family (Jakoby et al., 2002). Expression during seed development, specific binding to G-box-like ACGT elements of the albumin and cruciferin promoters, and in vivo regulation of these target genes have been demonstrated (Lara et al., 2003). A peculiarity exists in that none of the four genes in the bZIP group $\mathrm{C}$ in Arabidopsis shows seed-specific expression, in contrast with characterized members of the cereal O2-type TFs. This suggests that in Arabidopsis, combinatorial interactions and expression levels of different TFs may be of major relevance in the induction of seed-specific gene expression patterns.

ABSCISIC ACID INSENSITIVE3 (ABI3), FUSCA3 (FUS3), and LEAFY COTYLEDON2 (LEC2) have been implicated in seed development and belong to the B3 family of TFs (Parcy et al., 1994; Kroj et al., 2003; Braybrook et al., 2006; Santos-Mendoza et al., 2008). Mutations in any of these genes result in pleiotropic phenotypes affecting SSP accumulation and acquisition of desiccation tolerance. Recent genetic analysis demonstrated that these B3-type TFs are controlled by a complex self-regulating network including LEC1, LEC2, FUS3, and ABI3 (To et al., 2006). The corresponding genes clearly differ in expression and function despite being partially redundant. Although binding of $A B I 3$, FUS3, and LEC2 to the RY element, mediated by the B3 DNA binding domain, has been shown in vitro and in yeast systems (Kroj et al., 2003; Mönke et al., 2004; Braybrook et al., 2006), RY motifs are not sufficient to confer seed-specific expression patterns to a target promoter (Ezcurra et al., 2000; Nakashima et al., 2006). Cooperation with neighboring cis-elements and the cognate TFs has been demonstrated to be important to establish seed-specific transcriptional patterns. bZIP10 and bZIP25 physically interact with $A B I 3$, which in turn enhances in vitro DNA binding of the bZIP proteins to SSP promoters as well as their in vivo activation capacity (Lara et al., 2003). Therefore, cooperation of transcriptional regulators by protein-protein interactions provides an efficient mechanism to control gene expression in seeds and explains some of the molecular mechanisms underlying SSP gene expression. However, our knowledge on the protein partners and their interplay remains limited.

In general, bZIP TFs bind DNA as homo- or heterodimers (Ellenberger et al., 1992). Recently, we demonstrated that group $\mathrm{C}$ bZIPs, such as bZIP10 and bZIP25, preferentially interact with group S1 bZIPs, resulting in a set of specific heterodimers designated the C/S1 network of bZIP TFs (Ehlert et al., 2006). In particular, members of the $\mathrm{C} / \mathrm{S} 1$ network have been implicated in energy homeostasis (Baena-Gonzalez et al., 2007; Usadel et al.,
2008), amino acid metabolism (Weltmeier et al., 2006; Hanson et al., 2008), stress response (Kaminaka et al., 2006), and sinkspecific gene expression (Rook et al., 1998; Weltmeier et al., 2009). Importantly, these heterodimers were shown to convey synergistic activation properties to target genes, suggesting that heterodimerization serves as an efficient mechanism of signal integration (Weltmeier et al., 2006). With respect to the regulation of the seed gene $E m$, the importance of bZIP heterodimers and the $A B I 3$ othologous regulator VP1 has already been proposed in rice (Oryza sativa) (Nantel and Quatrano, 1996).

In this work, we aimed to identify S1 bZIP members that could be involved in seed gene regulation as important potential partners of previously described group $C$ bZIPs. We define the S1 TF bZIP53 as a key regulator of MAT gene transcription. bZIP53 enhances MAT gene expression by specific heterodimerization with bZIP10 or bZIP25. Furthermore, these bZIP heterodimers interact with $A B I 3$, which further increases MAT gene activation. Therefore, we propose that bZIP53 plays a pivotal and crucial role in quantitative control of MAT gene transcription levels by cooperation with several TFs forming enhanceosomelike protein complexes.

\section{RESULTS}

\section{bZIP53 Is Expressed in Seed Tissues during Maturation}

We have previously shown that Arabidopsis S1 bZIP proteins support strong heterodimerization with members of the group C bZIPs using two-hybrid systems in yeast and protoplasts and bimolecular fluorescence complementation (BiFC) techniques (Ehlert et al., 2006; Weltmeier et al., 2006). Since two group C members, bZIP10 and bZIP25, are known activators of SSP genes (Lara et al., 2003), we surveyed the expression patterns of S1 members during seed development to identify possible partners for C-S1 interactions in this process. According to the mRNA profiles of S1 bZIPs during silique and seed development derived from public AtGenExpress microarray data (http://www. genomforschung.uni-bielefeld.de/GF-research/AtGenExpressSeedsSiliques.html), bZIP53 is the S1 member with the highest expression in mid and late maturation phases. Induction of bZIP53 precedes and overlaps the activation of typical SSP and LEA genes, supporting the hypothesis that bZIP53 might be involved in MAT gene regulation.

Confirmation of the bZIP53 temporal pattern of expression and its localization within the seed were obtained by in situ hybridization studies (Figure 1A). bZIP53 mRNAs produced a strong signal in the embryo cotyledons during late maturation. In addition, we performed histochemical localization of $\beta$-glucuronidase (GUS) activity in seeds of Arabidopsis transgenic plants expressing the GUS reporter gene under control of the bZIP53 gene promoter (ProbZIP53:GUS). The results presented here were confirmed in three independent transgenic lines, all of which showed the same profiles of GUS expression. As seen in Figure 1B, GUS staining in the developing embryo was observed from the torpedo to the green cotyledon stages during seed development (Bowman, 1994). Altogether, these results demonstrate that bZIP53 expression increases during seed development and localizes to the embryo and endosperm during the maturation phase. 
A

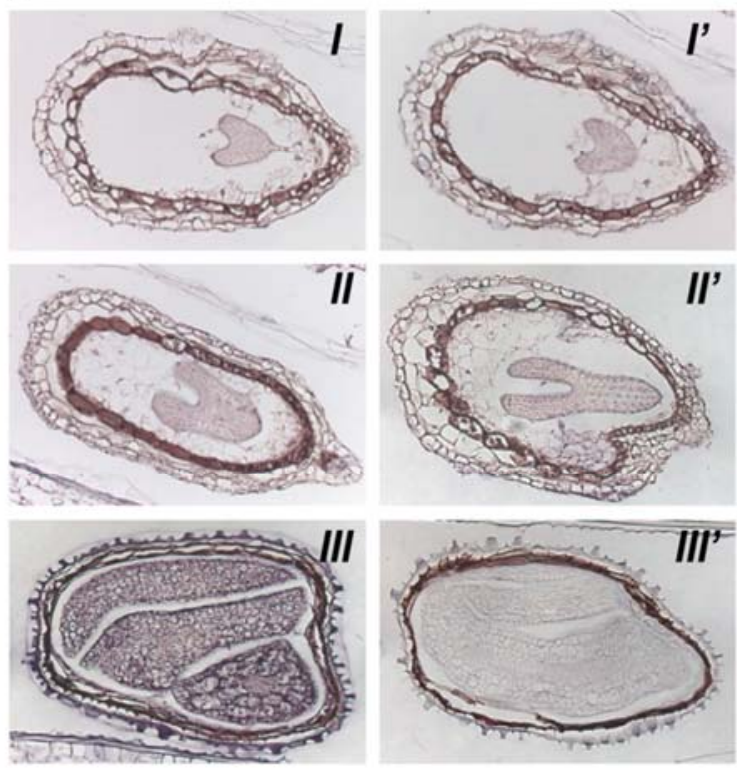

B

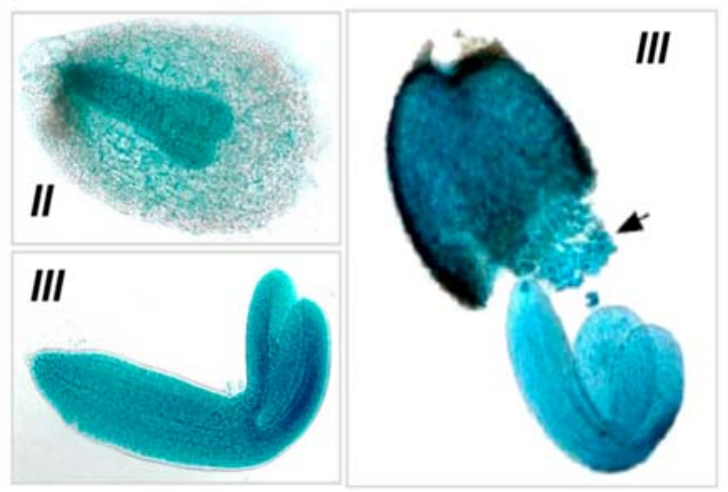

Figure 1. Expression of bZIP53 in Seeds.

(A) In situ mRNA hybridization for bZIP53 at different stages of seed development. Longitudinal sections of siliques with seeds at heart (I and I'), early torpedo (II and II'), and green cotyledon (III and III') stages of development are shown. Sections were hybridized with a bZIP53 antisense (I, II, and III) or a sense probe (I', II', and III').

(B) Histochemical analysis of Columbia (Col-0) seeds harboring a bZIP53 promoter fused to GUS (ProbZIP53:GUS). GUS staining of late torpedo stage (II) and green cotyledon stage (III) embryo development are shown. The arrow indicates the developing endosperm; to facilitate viewing, the seed has been pressed to push out the embryo.

\section{Ectopic Expression of bZIP53 Results in Abnormal Plant Growth and Expression of Seed-Specific Genes in Leaf Tissue}

Because of its particularly high levels of expression during seed development, bZIP53 could be expected to participate in seed gene regulation. To address this question, we followed a gain-of- function approach and obtained transgenic Arabidopsis plants with ectopic expression of bZIP53 driven by the cauliflower mosaic virus 35S promoter (Pro35S:bZIP53) (Weltmeier et al., 2006). As seen in Figure 2A, strong overexpression of bZIP53 resulted in phenotypic alterations, including dwarfism and delayed bolting compared with the wild-type plants. To test whether these modifications were associated to altered patterns of seed gene expression, mRNA isolated from wild-type and Pro35S:bZIP53 plants was subjected to quantitative RT-PCR (qRT-PCR) to check for bZIP53, MAT, and LEA gene expression. Figure $2 \mathrm{~B}$ shows data of wild-type and two independent Pro35S: bZIP53 transgenic lines with increased levels of bZIP53 mRNA as well as induced expression of seed MAT and LEA genes, indicating that bZIP53 is able to activate seed-specific expression in Arabidopsis leaves. Accumulation of SSP transcripts in leaves, as exemplified by the cruciferin (CRU3) and albumin (2S2) genes, was also obtained by overexpression of HA-tagged bZIP53 (see Supplemental Figures 1A and 1B online). The presence of the transgene-encoded protein was confirmed by
A
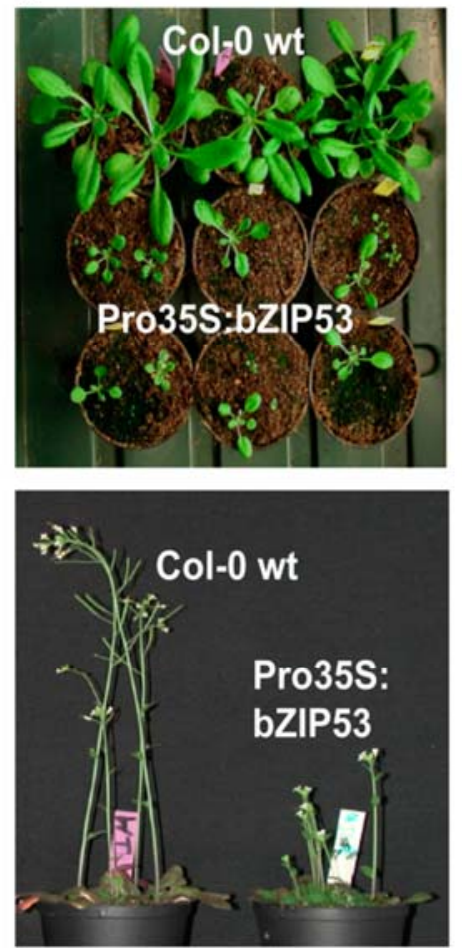

B
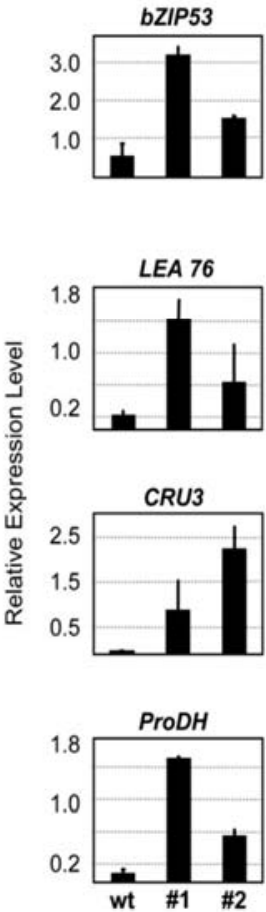

Figure 2. Plants with Constitutive Expression of bZIP53 Have Growth Defects and Increased Levels of Seed Maturation Transcripts.

(A) Plants overexpressing bZIP53 (Pro35S:bZIP53) have a stunted and late flowering phenotype compared with Col wild-type (Col-0 wt) plants. (B) mRNA samples from 2-week-old wild-type and two Pro35S:bZIP53 lines (\#1 and \#2) were analyzed by qRT-PCR to quantify the transcript levels of bZIP53, LEA76, CRU3, and ProDH. Expression levels are given relative to a UBIQUITIN gene for normalization. Given are mean values and standard deviation of two to three replicates. 
immunoblot analysis. Further examples of seed genes misexpressed in leaves are legumin-like CRA1 (Wang et al., 2007) and the 11- $\beta$-hydroxysteroid dehydrogenase gene (HSD1) (Li et al., 2007; see Supplemental Figure 1C online). In addition, these plants also show enhanced expression of the proline dehydrogenase $(\mathrm{ProDH})$ gene, as previously reported (Weltmeier et al., 2006), or the Asparagine Synthetase1 (ASN1) gene, both typical genes involved in amino acid metabolism (Lam et al., 2003) (Figure 2B; see Supplemental Figure 1C online). Altogether, these data support the assumption that overexpression of bZIP53 in leaves triggers the misexpression of a substantial fraction of seed-specific genes.

\section{The Albumin 2 S2 Gene Is a Direct Target of bZIP53}

To elucidate whether the SSP promoters were directly targeted by bZIP53, we performed chromatin immunoprecipitation (ChIP) from leaves of plants expressing ectopical bZIP53 tagged at the $\mathrm{N}$ terminus with a $3 \mathrm{xHA}$ epitope. These plants have been previously described and display the same phenotype as the untagged Pro35S:bZIP53 plants described in Figure 2A (Weltmeier et al., 2006).

Figure $3 \mathrm{~A}$ shows the results of qPCR analyses for the $2 \mathrm{~S} 2$ promoter on chromatin samples isolated from wild-type and Pro35S:HA-bZIP53 plants. The relative enrichment of the 2S2 gene promoter in Pro35S:HA-bZIP53 immunoprecipitated samples supports direct binding of a protein complex that includes bZIP53.

\section{Heterodimerization of bZIP53 with Group C bZIP10 or} bZIP25 Promotes Strong Activation of Seed-Specific Genes

Activation of seed-specific genes in leaves of Pro35S:bZIP53 plants as well as ChIP analyses suggest that bZIP53 is directly involved in this regulation. We studied the activation properties of bZIP53 in Arabidopsis leaves transformed by particle bombardment (Lara et al., 2003). Figures 3B and $3 \mathrm{C}$ show an example of $2 S 2$ promoter constructs driving the expression of a GUS reporter that is cotransformed with effector plasmids for bZIP53, bZIP10, and bZIP25. Individually, none of these bZIPs is able to produce a significant increase in the basal activity of the reporter. Previous studies showed no significant heterodimerization for bZIP10 and bZIP25, whereas strong heterodimerization of group C bZIPs and bZIP53 was reported (Ehlert et al., 2006). Accordingly, when bZIP53 was cotransformed with bZIP10 or bZIP25, a dramatic increase in the reporter activity was observed. These results were confirmed in transiently transformed leaf mesophyll protoplasts using two different seed-specific reporter constructs, driven by the $2 S 2$ or the CRU3 promoter (see Supplemental Figure 2 online). Similarly, immunoblot analysis confirmed expression of the bZIP genes (see Supplemental Figure 3A online) in transient assays in protoplasts. Unexpectedly, we observed that coexpression of bZIP10 and bZIP53 leads to enhanced protein levels, suggesting that heterodimer formation might stabilize the bZIP proteins from degradation. This data emphasize the importance of heterodimer formation between bZIP53 and group C bZIPs in the observed enhancement of gene activation.
Activation of the 2S2 Seed Storage Protein Gene by bZIP53 and Its Heterodimers Relies on the G-Box Promoter Element

The in vivo regulation of bZIP53 and its heterodimers was further investigated on the $2 \mathrm{~S} 2$ promoter. A modified version of the native promoter was used, in which the G-box element was mutated to a sequence that prevents bZIP DNA binding (Figure 3B; Lara et al., 2003). Different from the behavior of the native promoter, the activation mediated by the bZIP TFs is completely abolished in this mutant (Figure $3 \mathrm{C}$ ), indicating that the regulation of the $2 \mathrm{~S} 2$ promoter by these bZIP proteins requires an intact G-box motif.

\section{bZIP53 Binds in Vitro to the G-Box Present in the 2S2 Promoter, and Heterodimerization with bZIP10 or bZIP25 Increases Its Binding Activity}

To establish if bZIP53 was able to bind to seed-specific gene promoters, we performed in vitro DNA binding experiments with an epitope-tagged bZIP53 (T7-bZIP53) protein. A nativesequence oligonucleotide (wild type) containing a functional bZIP (G-box) binding site (Ezcurra et al., 2000) derived from the 2S2 promoter and a mutated version were used (Figure 3B). Doublestranded oligonucleotides were covalently attached to 96-well ELISA plates before doing the binding assays as described in Methods. Figure 3D shows a positive correlation between the amount of T7-bZIP53 detected with the epitope-specific antibody and the amount of T7-bZIP53 protein extract added to the wells, indicating T7-bZIP53 binding to the wild-type oligonucleotide in a concentration-dependent manner. To analyze the specificity of this binding, competition experiments were performed with increasing amounts of the wild-type or mutated oligonucleotides added to the binding reactions. As shown in Figure 3D, the wild-type, but not the mutated oligonucleotide, was able to reduce the amount of T7-bZIP53 bound to the plate, thus confirming the specificity of the binding.

We have shown in previous work and in this study that bZIP53 heterodimerization with bZIP10 or bZIP25 increases its transactivation properties (Ehlert et al., 2006; Weltmeier et al., 2006). To determine if the heterodimerization was also affecting the binding capabilities of these proteins, we incubated increasing amounts of T7-bZIP53 in the wild-type oligo-coated wells in the presence or absence of fixed amounts of bZIP10. Figure $3 E$ illustrates the effect of heterodimerization between bZIP53 and bZIP10 and shows that the binding activity for the concentration series of bZIP53 is higher in the presence (than in the absence) of bZIP10. Likewise, the same behavior is observed in the reciprocal experiment for a bZIP10 series, in support of an increased binding activity of the heterodimers.

\section{ABI3 Does Not Interact with bZIP53 Alone, but Heterodimers with bZIP10 or bZIP25 Form a Ternary Complex in Yeast and in Planta}

$A B \mid 3$ is an important regulator of gene expression in seeds of Arabidopsis (Giraudat et al., 1992; Parcy et al., 1994) and is able to interact with bZIP10 or bZIP25 to increase their activation capacity on the 2S2 promoter (Lara et al., 2003). We used a yeast 
A

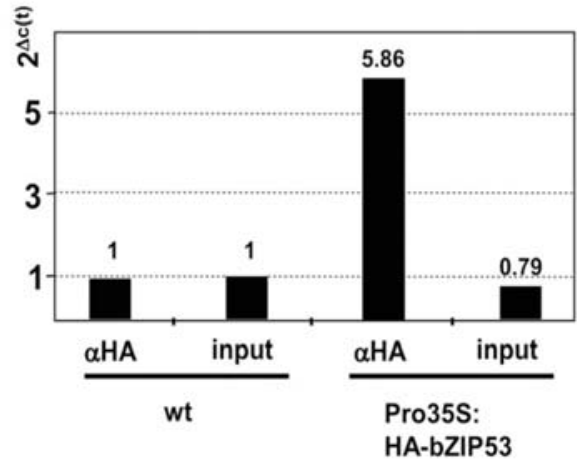

B

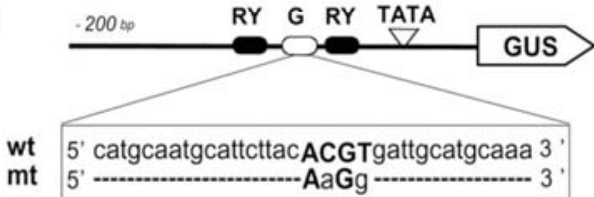

C

Effectors
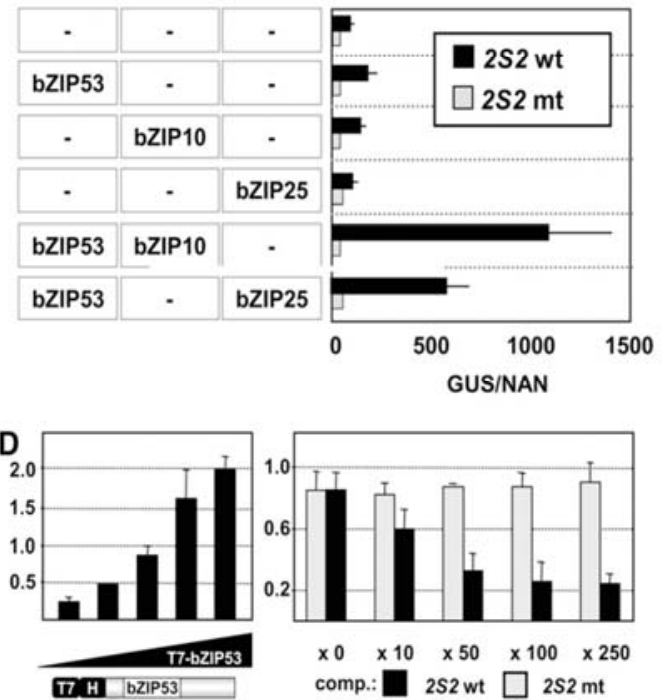

E

$\rightarrow T 7$-bZIP53

- o- T7-bZIP53 +bZIP10

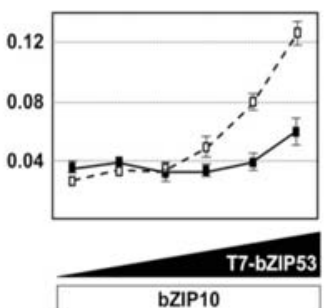

- 0 - T7-bZIP10

$\rightarrow-T 7$-bZIP10 +bZIP53

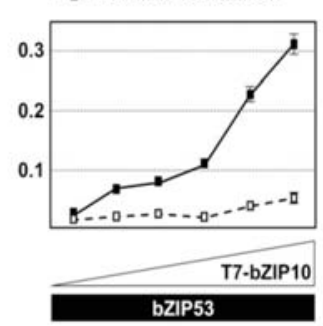

Figure 3. In Vivo and in Vitro Binding of the bZIP53 Protein to the Albumin 2S2 Promoter and Interaction with bZIP10 and bZIP25.

(A) Chromatin extracts from wild-type plants and plants overexpressing a HA-tagged bZIP53 protein (Pro35S:HA-bZIP53) were subjected to qRT-PCR analysis with $2 S 2$ promoter-specific primers before (input) and two-hybrid system to determine if bZIP53 was also able to interact with $A B I 3$. We expressed the $A B I 3$ and bZIP53 proteins as fusions to the GAL4 binding (BD) or activation (AD) domains, respectively, and introduced them into yeast strains containing LacZ or HIS reporter genes under the control of GAL4 binding sites. Different combinations of $\mathrm{ABI} 3$ and bZIP53 were tested for possible interactions in this system, alongside $\mathrm{ABI}$, another bZIP TF that has been implicated in seed gene expression serves here as a negative control (Finkelstein and Lynch, 2000). As seen in Figure 4A, no interaction could be detected between bZIP53 and $A B \mid 3$ or between bZIP53 and ABI5 in any of the experimental systems used. As previously reported, positive interactions between bZIP53 and bZIP10 or bZIP25 confirmed their capacity to heterodimerize. To further analyze the possibility that these heterodimers could still interact with $A B \mid 3$, three-hybrid system experiments were devised. Yeast cells expressing BD-bZIP53 were cotransformed with either AD-bZIP10 or AD-bZIP25 in the presence or absence of $A B \mid 3$, and $\beta$-galactosidase reporter

after immunoprecipitation with an anti-HA antibody $(\alpha-\mathrm{HA})$. Ct values for Pro35S:HA-bZIP53 samples were subtracted from the $\mathrm{Ct}$ values of the equivalent wild type, and the differentials are shown on top of the right bars in the graph. A value of 1 was assigned to the Col-0 samples. For normalization, an actin (ACT7) gene was used (see Methods).

(B) Schematic view of the $2 S 2$ promoter fused to GUS used as reporter in transient expression analysis. Depicted are RY (black) and G-box (white) elements. The sequence of the wild type and mutated G-box $(\mathrm{mt})$ is shown.

(C) Arabidopsis leaves were transformed with the reporter constructs containing sequences described in (B) and effector constructs containing specific bZIP genes under control of the Pro35S in cobombardment experiments as described by Lara et al. (2003). Three microliters of control plasmid Pro35S:NAN was included in all the experiments to normalize GUS expression values for differences in bombardment efficiencies (Kirby and Kavanagh, 2002). The $x$ axis values are expressed as GUS activity relative to NAN. Given are mean values and standard deviation of three independent experiments.

(D) In vitro binding of bZIP53 to the G-box sequence from the 2S2 promoter. A biotinylated oligonucleotide containing the G-box sequence was bound to streptavidin-coated wells and incubated with increasing amounts of a T7-tagged bZIP53 protein (1 to 1:90). Nonbound proteins were removed from the reaction wells, and the amount of T7-bZIP53 protein was quantified by immunodetection with an anti- $T 7$ antibody $(\alpha-T 7$; left panel). The binding specificity of bZIP53 to the $2 S 2 \mathrm{G}$-box was analyzed in competition experiments where increasing amounts of unlabeled oligonucleotides (as indicated) containing a wild-type (2S2 $w t$; black bars) or a mutated $2 \mathrm{~S} 2 \mathrm{mt}$ version (gray bars) were incubated with a fixed amount of the T7-bZIP53 protein and the biotinylated oligonucleotide containing the wild-type G-box sequence (right panel). (E) Effect of heterodimerization on bZIP53 and bZIP10 binding affinity. A biotinylated oligonucleotide containing the G-box sequence was bound to streptavidin-coated wells and incubated with increasing amounts ( 1 to 1:30) of a T7-bZIP53 protein in the absence (filled squares) or presence of a fixed amount of bZIP10 protein (open squares). Nonbound proteins were removed from the reaction wells, and the amount of T7-bZIP53 protein was quantified by immunodetection with an $\alpha-T 7$ antibody (left panel). The reciprocal experiment was performed with a T7-bZIP10 protein and a nontagged bZIP53 protein (right panel). Given are mean values and standard deviation of three repetitions. 
A

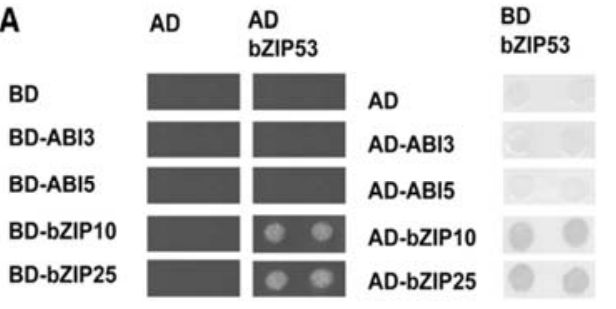

B

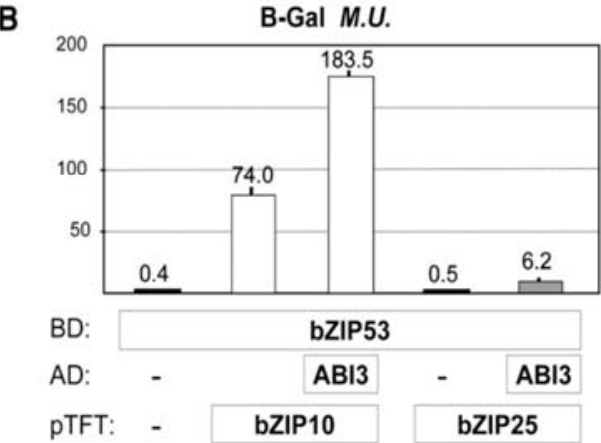

C
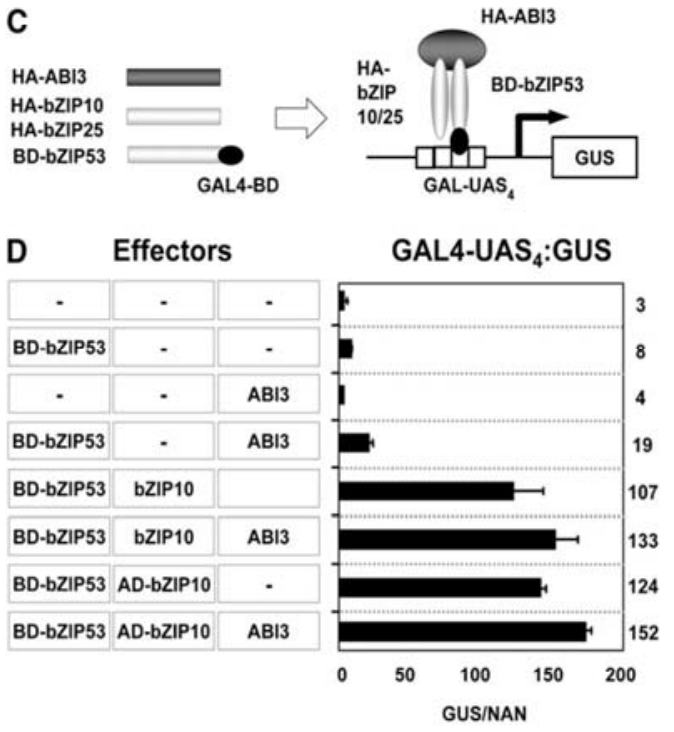

Figure 4. Interaction of bZIP53, bZIP10, and bZIP25 Homo- or Heterodimers with $A B I 3$

(A) Protein interactions in yeast two- and three-hybrid systems. ABI3, $A B 15, b Z I P 10$, and bZIP25 proteins were fused to the GAL4 DNA BD or $A D$ and introduced separately into a yeast strain containing the $A D$ bZIP53 (HF7c) or BD-bZIP53 (SFY526) protein, respectively. Activation of the reporter genes HIS3 (growth in a His-depleted medium; left panel) and LacZ (blue colored colonies; right panel) indicates positive proteinprotein interactions.

(B) Yeast strains (SFY526) expressing different combinations of BDbZIP53, AD-ABI3, and bZIP10 or bZIP25 were assayed for $\beta$-galactosidase activity. The latter were provided in the three-hybrid vector PTFT (Egea-Cortines et al., 1999). Average values (Miller units) and standard errors from six replicates and two independent experiments are shown. (C) Schematic overview of a three-hybrid assay in Arabidopsis proto- activities were quantified (see Supplemental Figure 4 online). As previously observed, the interaction between BD-bZIP53 and either AD-bZIP10 or AD-bZIP25 was confirmed by an increase of the reporter activity. However, when a construct designed to express $\mathrm{ABI} 3$ was cotransformed into the strains, a further increase of the $\beta$-galactosidase activity was observed, in support of a trimeric interaction. As expected for a negative control, the bZIP factor ABI5, which interacts with $A B I 3$ (Nakamura et al., 2001) but does not heterodimerize with AtbZIP53, did not show activation indicative of ternary complex formation.

Reciprocal three-hybrid analysis where the noninteracting proteins bZIP53 and ABI3 were fused to the Gal4BD and Gal4AD, respectively, showed also an increase of reporter activity when assayed in the presence of bZIP10 or bZIP25 (Figure 4), in support of the proposed trimeric interaction. Altogether, these results indicate that $\mathrm{ABI} 3$ can be brought into the heterodimer complex, probably by its interaction with bZIP10 and/or bZIP25. Importantly, bZIP53 does not interfere with the interaction between ABI3 and bZIP10 or bZIP25.

Ternary complex formation in plant cells was supported by a similar three-hybrid approach developed in Arabidopsis protoplasts. Figure $4 \mathrm{C}$ shows a schematic overview of the experimental setup and the structure of the expressed proteins used to transactivate a reporter construct containing the GUS gene under the control of a minimal promoter bearing a tetramer of the GAL4 upstream activating sequence (GAL4-UAS 4 :GUS) (Ehlert et al., 2006). As shown in Figure 4D, comparable results to the yeast system were achieved. Transactivation of the reporter with BD-bZIP53 alone results in low levels of GUS activity, whereas cotransformation with either HA-bZIP10 or ADbZIP10 displayed enhanced activity indicative of heterodimer formation as previously observed in the yeast two-hybrid system. Expression of the corresponding proteins was assayed by immunoblot analysis with $\mathrm{HA}$-tag and BD-specific antibodies (see Supplemental Figure 3B online). Equally, in both systems, the supplementary inclusion of $\mathrm{HA}-\mathrm{ABI} 3$ resulted in an additional increase of reporter activity, in support of its capacity to interact with the heterodimers in plant cells.

BiFC (Walter et al., 2004) was used as an additional experimental system to verify the detected ternary interactions in plant cells. bZIP53 and ABI3 proteins fused to different domains of yellow fluorescent protein (YFP) were coexpressed in onion cells in distinct combinations, rendering no reconstruction of YFP activity. However, when a third construct expressing bZIP10 protein was cotransfected, YFP fluorescence was observed in support of a tethering function of this protein to promote a ternary complex formation (Figure 5).

plasts. Constructs are shown on the left, and a model of reporter gene activation is shown on the right.

(D) Activation of the GAL4-UAS :GUS reporter after cotransfection with the constructs indicated in (C). The $x$ axis values are expressed as GUS activity relative to NAN (Ehlert et al., 2006). Average values and standard errors from four transfections are shown. Numbers along the $y$ axis represent fold induction values relative to nontransfected control cells. The experiments were repeated three times with similar results. 
A
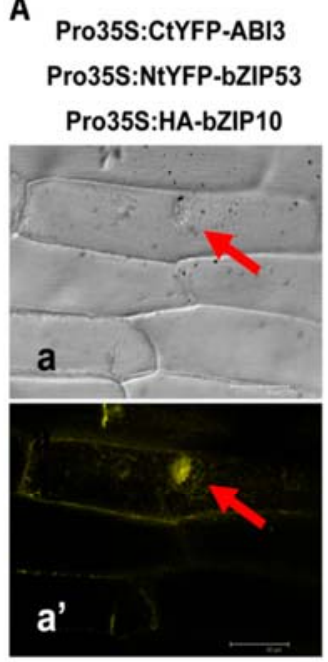

B
Pr35S:CtYFP-ABI3 Pro35S:NtYFP-bZIP53 Pro35S:HA- $\varnothing$

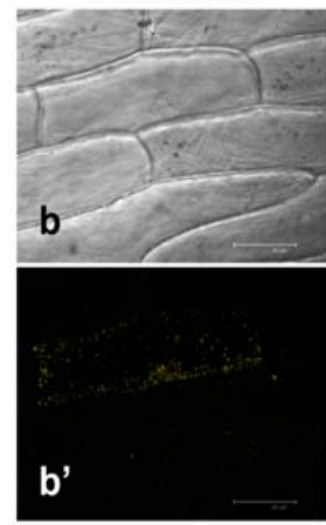

Figure 5. bZIP53, bZIP10, and ABI3 Protein-Protein Interaction Studied by BiFC (Walter et al., 2004).

Onion epidermis cells have been transiently transformed by particle bombardment. Fusion proteins of the C-terminal part of YFP (CtYFP) and $\mathrm{ABI} 3$ and the N-terminal part of YFP (NtYFP) and bZIP53 have been coexpressed with HA-tagged bZIP10 (HA-bZIP10) (a and a') or an empty vector control ( $\left(\mathrm{b}\right.$ and $\left.\mathrm{b}^{\prime}\right)$. Bright-field images (top panels) and epifluorescence images taken by confocal microscopy (bottom panels) are shown.

Heterodimer Formation and Interaction with $\mathrm{ABI}$ ( Regulate the Activation Properties of bZIP53 on SSP Gene Expression in Plant Cells

To study the functional implications of the interaction between $\mathrm{ABI} 3$ and bZIP heterodimers in plant cells, we performed transient expression analysis in Arabidopsis protoplasts using two different SSP promoters, corresponding to the 2S2 and CRU3 genes. Figure $6 \mathrm{~A}$ shows that the use of bZIP effectors individually or in combination with $\mathrm{ABI} 3$ resulted in limited activation capacity on both promoters. Combination of bZIP53 and bZIP10 led to an important increase in the activity, in accordance with previous results. Moreover, the inclusion of $\mathrm{ABI} 3$ in this combination produced an additional increase in the activation of both promoters.

This reporter gene activation cannot be observed when using an effector construct expressing bZIP10 with two Pro exchange mutations (bZIP10pp); these mutations disrupt the zipper dimerization surface and therefore abolish the interaction with bZIP53 (Weltmeier et al., 2006). Expression of bZIP10pp was found to be comparable to the wild-type protein as confirmed by immunoblot analysis (Figure 6B). Accordingly, disruption of the bZIP heterodimer using bZIP10pp abolishes this induced gene activation both in the presence or absence of $A B \mid 3$. These results indicate a functional interaction between bZIP53/bZIP10 heterodimer and $\mathrm{ABI} 3$ and confirm a positive effect of $\mathrm{ABI} 3$ on heterodimermediated transcription.

To further investigate this effect, a comprehensive analysis of $\mathrm{ABI} 3$ action on the heterodimer was performed. In Figure $6 \mathrm{C}$,

different ratios of bZIP53 and bZIP10 were used to transactivate the 2S2 promoter in Arabidopsis protoplasts, either in the presence or in the absence of $A B I 3$. In all cases, the activation effect of the heterodimer was enhanced in the presence of $\mathrm{ABI} 3$ by a similar increment. However, maximum promoter activity was greatly determined by the ratio of the heterodimerization partners. A 1:1 ratio, which enables optimal heterodimerization, results in maximum promoter activation.

\section{bZIP53 Loss-of-Function Plants Display Reduced Expression of SSP Genes}

Results from the analysis of ectopic expression of bZIP53 suggested a regulatory role in SSP gene expression. We used a complementary loss-of-function strategy making use of a bzip53 T-DNA insertion mutant (Weltmeier et al., 2006) to corroborate the functional relevance of bZIP53 for SSP expression. We quantified SSP gene expression in bzip53 plants during seed development. Siliques of wild-type and bzip53 plants were collected at different stages of seed development, and total RNA was isolated and used in qRT-PCR experiments. Figure 7A shows that levels of bZIP53 mRNA were greatly reduced throughout all the stages of silique development in the bzip53 plants. However, residual levels of bZIP53 were detected, probably due to the leaky nature of the bzip53 mutation since the T-DNA is inserted in the promoter region (Weltmeier et al., 2006). Observed differences in the timing of RNA accumulation between CRU3, 2S2, and LEA76 genes were in accordance with those previously described for MAT and LEA genes (Parcy et al., 1994). Comparisons between wild-type and bzip53 plants show significantly reduced levels of SSP transcript in the latter. Although the timing of mRNA accumulation for these genes in the bzip53 samples was not significantly altered, the mRNA levels were reduced throughout the maturation stages and no peaks of RNA accumulation were detected, in contrast with the wild-type samples (Figure 7A).

As seen above, impaired bZIP53 activity leads to decreased SSP gene expression in the seed, probably by limiting the activation capacity of the regulatory complex involving bZIP10/ bZIP25 and ABI3. To test this hypothesis, we performed transactivation experiments using a $2 S 2$ promoter driving a GUS reporter in different plant backgrounds. Figure 7B shows results of particle bombardment experiments on Arabidopsis leaves from wild-type, Pro35S:bZIP53, and bzip53 plants. As previously reported (Lara et al., 2003), the use of bZIP10 and $\mathrm{ABI} 3$ as effectors in this system resulted in the activation of the $2 S 2$ promoter. This activation was significantly enhanced in Pro35S:bZIP53 plants and reduced in the bzip53 knockdown mutant line, consistent with the participation of bZIP53 in this regulatory complex. The same results were obtained for bZIP25 in combination with ABI3 (see Supplemental Figure 5 online).

\section{DISCUSSION}

Seed gene expression relies on specific TFs acting in a combinatorial fashion. Both in monocot and dicot species, group $\mathrm{C}$ 
A

Effectors

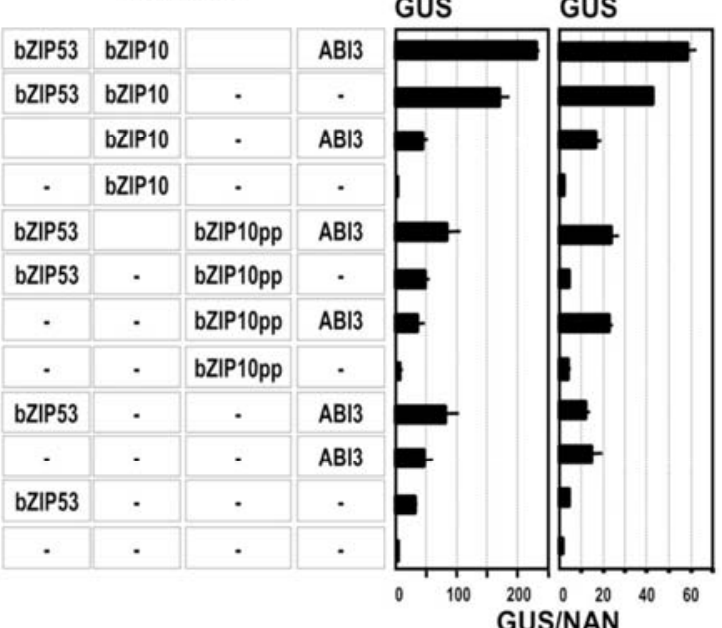

B
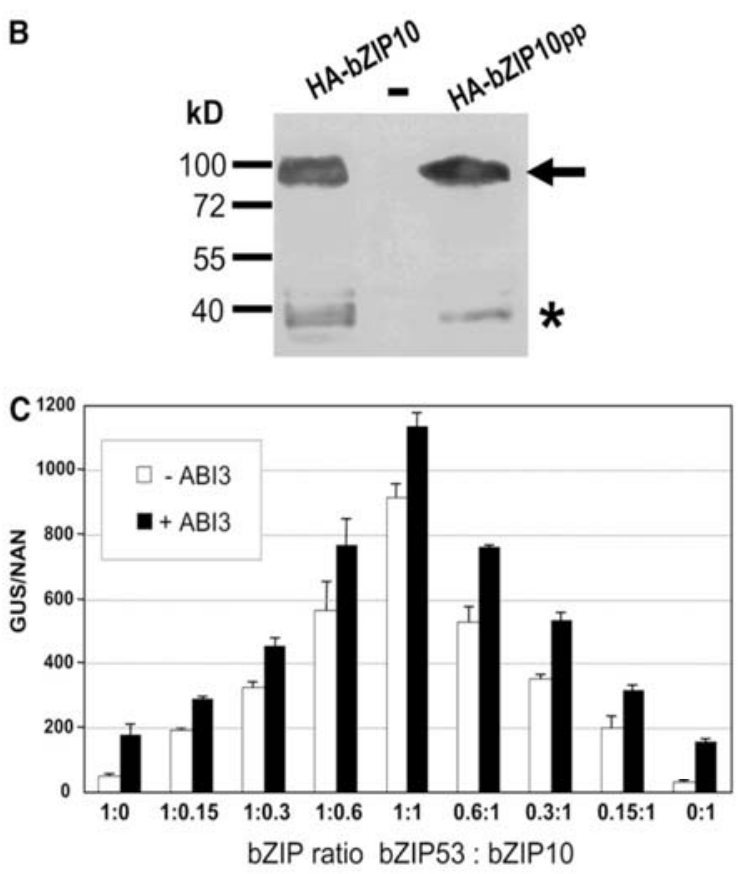

Figure 6. Effect of $A B \mid 3$ on the Transcriptional Activation Mediated by the bZIP53/bZIP10 Heterodimers.

(A) GUS reporter activity under the control of the $2 S 2$ or CRU3 promoter was measured in transiently transformed Arabidopsis protoplasts after cotransfection of the effector constructs indicated. bZIP10, bZIP53, and $\mathrm{ABI} 3$ are expressed under the control of Pro35S. For immunodetection, 3xHA-epitope-tagged derivatives were used (see Supplemental Figure 3 online). The importance of bZIP heterodimerization was demonstrated by including bZIP10pp, which is impaired in zipper-mediated dimerization (Weltmeier et al., 2006). Given are mean values and standard deviation of four independent transfections.

(B) Immunoblot analysis of transiently transformed protoplasts confirms that expression of HA-bZIP10 is comparable to HA-bZIP10pp-untransformed control protoplasts. HA epitope-tagged proteins were detected
bZIP factors related to maize $\mathrm{O} 2$ have been shown to be important players in this regulation. However, expression of the corresponding Arabidopsis bZIP genes is not seed specific. The recently described functional interaction between group $\mathrm{C}$ and S1 bZIPs (Ehlert et al., 2006) prompted us to investigate the possible participation of $\mathrm{S} 1$ members in seed gene regulation. In this study, we identified bZIP53 as the major S1 member expressed during seed maturation. Evidence of its participation in seed gene regulation was obtained by different approaches, including the analysis of plants with bZIP53 gain and loss of function. Moreover, functional studies show that bZIP53 plays a key role in the strong activation of seed-specific genes, directly involved in a seed regulatory protein complex encompassing the group $\mathrm{C}$ bZIP10 and bZIP25 and the $\mathrm{B} 3$ seed regulator $\mathrm{ABI} 3$.

\section{bZIP53 Constitutes a Transcriptional Regulator of Seed Maturation Genes}

Analyses of transcriptomic data of S1 bZIPs from repository databases show an increase in expression of bZIP53 (and to a lesser extent for bZIP1) during intermediate and late stages of seed development. This suggests a prominent role of bZIP53 in regulating seed gene expression in conjunction with the group $\mathrm{C}$ members. We further confirmed the precise pattern of expression of bZIP53 in seed tissues by mRNA in situ hybridization and promoter reporter fusions in transgenic Arabidopsis plants.

Gain-of-function approaches in transgenic plants and Arabidopsis leaf protoplasts ectopically expressing bZIP53 demonstrate that bZIP53 is able to activate several seed MAT and LEA genes, such as 2S2, CRU3, CRA1, HSD1, and LEA76. However, as observed before, the presence of MAT RNA does not necessarily result in the detection of the corresponding proteins. Additional cellular requirements and tissue-specific protein degradation determine high-level accumulation of MAT proteins (Gruis et al., 2004)

Complementing the gain-of-function results, reduced expression of bZIP53 in a knockdown line that harbors a T-DNA insertion in the promoter leads to strong reduction in the expression of the corresponding target genes. However, these plants still set viable seeds, which might be due to a residual amount of bZIP53 protein. The lack of complete knockout lines in mutant collections might be explained by an essential function of this gene. Alternatively, other genes among the 75 bZIP TFs identified in Arabidopsis (Jakoby et al., 2002) may partly substitute for bZIP53 function. Within the group S1, which harbors the closest homologs of bZIP53, only bZIP1 is intermediately induced during seed maturation with timing comparable to

using an $\alpha-\mathrm{HA}$ antiserum. The arrow indicates HA-bZIP10 and bZIP10pp proteins, and the asterisk shows degradation products.

(C) Transient expression using a GUS reporter gene under the control of the 2 S2 promoter. Combinations of bZIP53 and bZIP10 at different ratios (indicated are ratios of input DNA) in the presence (black bars) or absence (white bars) of $A B I 3$ were used as effectors. The $x$ axis values are expressed as GUS activity relative to NAN as described by Ehlert et al. (2006). Average values and standard errors from four transfections are shown. 
A
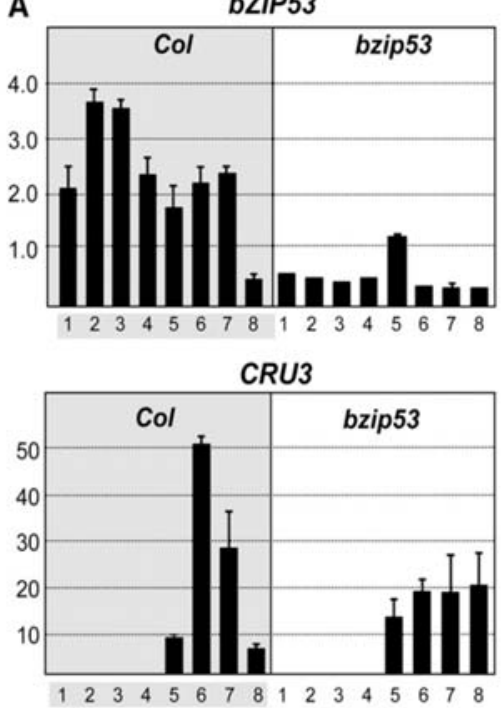

2S2

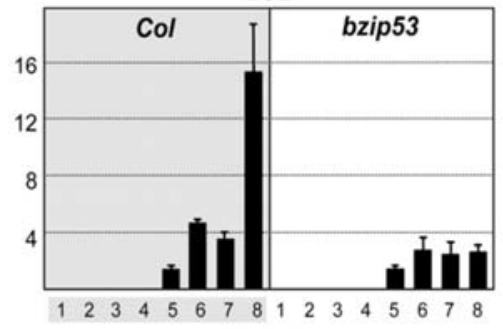

LEA76

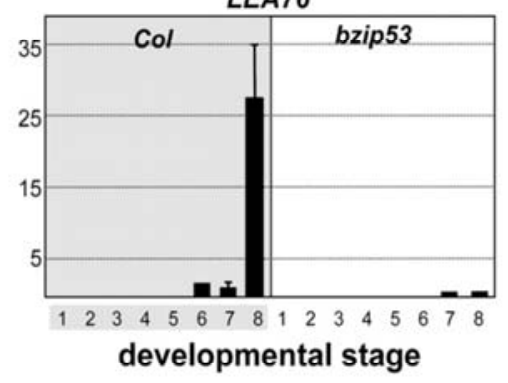

B

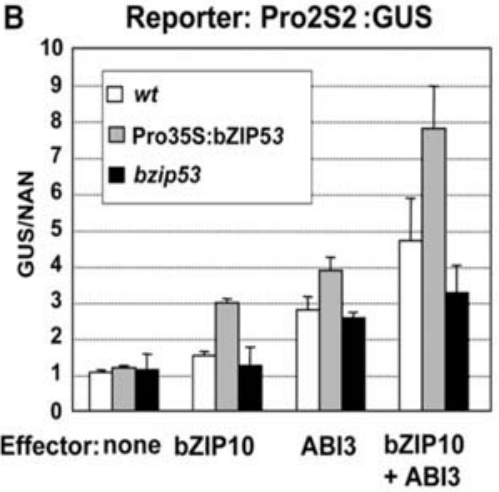

Figure 7. Effect of a bZIP53 T-DNA Insertion Mutant on Seed-Specific Gene Regulation.
bZIP53. However, ectopic overexpression of At bZIP1 does not lead to activation of MAT genes, indicating a lack of functional redundancy of these genes in MAT gene regulation (Weltmeier et al., 2009).

High-level ectopic expression of bZIP53 results also in a dwarf growth phenotype in vegetative phases. Based on these observations, it can be assumed that regulation of SSP genes reflects only a subset of bZIP53 targets. In previous work, we and others demonstrated that bZIP53 is also involved in regulating hypoosmolarity responses (Satoh et al., 2004; Weltmeier et al., 2006). In particular, the promoter of the ProDH gene, which is involved in regulating amino acid metabolism, has been defined as an in vivo target of bZIP53. Furthermore, a second amino acid metabolism gene, ASN1, was shown to be regulated by bZIP53 (this study) and other members of the $\mathrm{C} / \mathrm{S} 1$ network (Baena-Gonzalez et al., 2007; Hanson et al., 2008). Amino acid synthesis, transport, and SSP synthesis are linked processes that have to be coordinated in vegetative tissues as well as in the seed. In accordance, ectopic overexpression of ASN1 enhances the SSP content and nitrogen status of the seed (Lam et al., 2003). Further studies are necessary to test whether bZIP53 is coordinating other pathways in addition to SSP synthesis, such as amino acid metabolism during seed and vegetative development.

bZIP53 expression is not restricted to the seed and can be observed in vegetative tissues, particularly under certain stress conditions. Interestingly, salt treatment dramatically induces bZIP53 transcription in roots (Weltmeier et al., 2009). In the context of the seed, it is worth mentioning that besides storage compound accumulation, dehydration occurs during late stages of the maturation phase. Accordingly, stress-related programs of gene expression are also important in the establishment of desiccation tolerance. We have determined that bZIP53 can also control the expression of the seed-specific gene LEA76. Although our knowledge of LEA76 function is limited, LEA proteins are known to accumulate in seed and vegetative plant tissues following environmental stress (Xu et al., 1996) and also in desiccation-tolerant bacteria and invertebrates (Bies-Etheve et al., 2008; Hundertmark and Hincha, 2008). The hypothesis whether bZIP53 is involved in establishing stress tolerance in vegetative tissues requires further testing.

(A) mRNA levels detected of the seed maturation genes indicated by qRT-PCR in Col wild-type and bzip53 siliques at different stages of development. Lane 1, 0 d after flowering (DAF); lane 2, 2 DAF; lane 3, 4 DAF; lane 4, 6 DAF; lane 5, 9 DAF; lane 6, 12 DAF; lane 7, 15 DAF; lane 8 , $\geq 18$ DAF. Data are normalized using UBIQUITIN expression values. Average values and standard errors from at least two technical replicates are shown.

(B) Transient expression by microparticle bombardment of Arabidopsis leaves from the wild type (white bars) and plants with increased (Pro35S: bZIP53; gray bars) or decreased (bzip53; black bars) expression of bZIP53. Effector constructs containing bZIP10 or ABI3 under the control of a $35 \mathrm{~S}$ promoter (Pro35S) and a GUS reporter gene under the control of the $2 S 2$ promoter were used. The $x$ axis values are expressed as GUS activity relative to NAN (Kirby and Kavanagh, 2002). Average values and standard errors from four replicates and two different experiments are shown. 


\section{Specific Heterodimerization of bZIP53 and Group C bZIPs Executes an Efficient Mechanism to Enhance Transcription of Seed Maturation Genes}

MAT gene regulation is one of the early investigated areas in plant transcriptional control. The importance of G-box ciselements in MAT gene promoters has been determined both in monocot and dicot species (Vicente-Carbajosa and Carbonero, 2005; Santos-Mendoza et al., 2008), and its regulation by seedspecific O2-type bZIPs has been firmly established in cereals. By contrast, genome scale transcriptomic data in Arabidopsis do not reveal a seed-specific candidate among all bZIPs, indicating that in this species (and probably in nonendospermic seeds from dicot plants), combinatorial effects are likely of major importance in the regulation of seed gene expression. In this framework, the group $\mathrm{C}$ bZIPs related to $\mathrm{O} 2$, bZIP10, and bZIP25 are able to interact with $\mathrm{ABI} 3$ and have been proposed as Arabidopsis counterparts of the monocot bZIP seed regulator (Schmidt et al., 1990; Lara et al., 2003). The corresponding genes, which are not seed specific, are constitutively expressed at low levels in other tissues, and bZIP10 has been implicated in plant stress responses to pathogen attack in vegetative tissues (Kaminaka et al., 2006). Previous work has defined a specific heterodimerization network of group $\mathrm{C}$ members with group $\mathrm{S} 1 \mathrm{bZIPs}$, such as bZIP53 (Ehlert et al., 2006). The expression of this gene increases during seed maturation, and it strongly heterodimerizes with bZIP10 and bZIP25 as demonstrated in yeast and plant protoplasts (Ehlert et al., 2006), as well as by BiFC. Additionally, in vitro binding studies show that they can bind DNA as heterodimers (Weltmeier et al., 2006).
Efficient methods for in vivo DNA binding studies in Arabidopsis seeds have not been established. However, here, we demonstrated binding of bZIP53 to the 2S2 promoter using leaf-derived chromatin from Pro35S:HA-bZIP53 plants, which in turn show vegetative transcription of the $2 \mathrm{~S} 2$ gene. Although this approach does not unequivocally confirm participation of bZIP53 in regulation of MAT genes, it provides circumstantial evidence in line with other results presented in this study. Mutational approaches confirm the $2 \mathrm{~S} 2 \mathrm{G}$-box as the in vitro binding site and also as the relevant in vivo cis-regulatory element. At high protein concentration, bZIP53 displays interaction with G-box elements in vitro, but titration experiments demonstrate that binding of bZIP53 in the presence of bZIP10 or bZIP25, presumably by heterodimer formation, is significantly enhanced. The in vivo relevance of heterodimer formation is further supported by expression of bZIP mutants, which are impaired in zipper-mediated dimerization, and consequently target gene activation. Detailed titration experiments in protoplasts also demonstrate that the level of target gene expression is determined by the ratio of the bZIP heterodimerization partners. The 1:1 ratio of bZIP53 and bZIP10 leads to the highest target gene activity. Altogether, these data provide conclusive evidence that bZIP heterodimers show enhanced binding to and gene activation through G-box-like ciselements. Importantly, heterodimerization provides an efficient mechanism by which bZIP53 drives MAT gene expression.

Possible mechanisms of heterodimer-enhanced transactivation have been described elsewhere (Weltmeier et al., 2006), including (1) increased affinity for binding target sites, (2) stabilization of dimer structures (as effective DNA binding forms) via

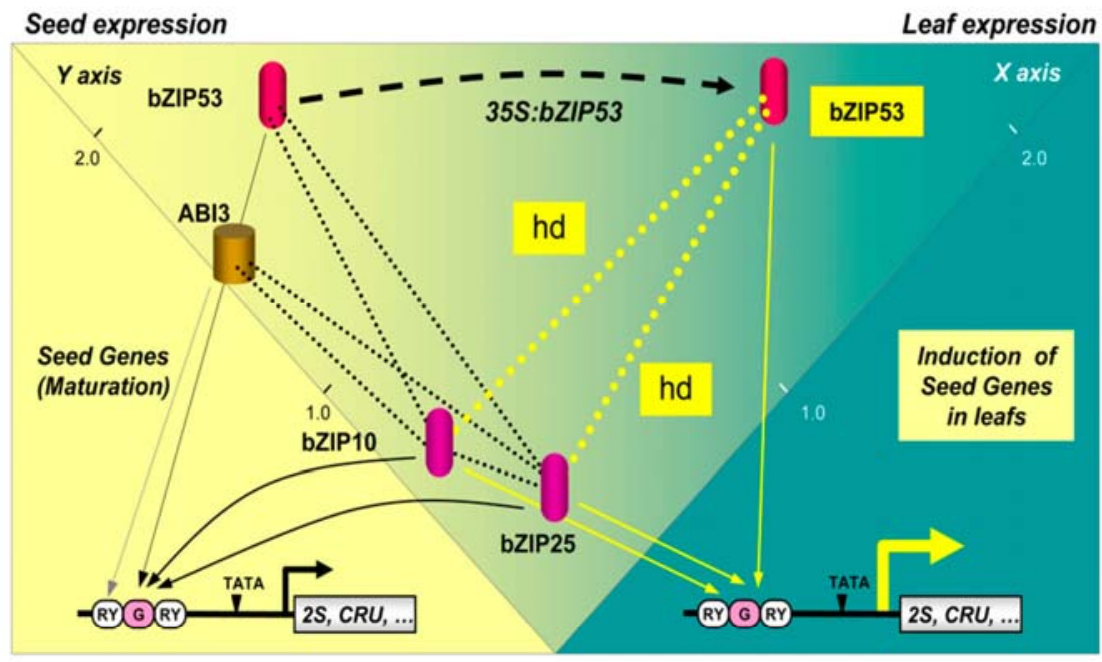

Figure 8. Model for bZIP53 Regulation of Seed Maturation Gene Expression.

Yellow and blue backgrounds represent expression in the seed and leaf, respectively. Positions of the indicated TFs were represented in a coordinate system according to relative expression values in seeds $(y$ axis) and leaves ( $x$ axis) (data derived from AtGenExpress). Structure of a typical SSP promoter-like albumin (2S2) or cruciferin (CRU3) is depicted, indicating regulatory elements in their promoters (RY, RY-box; G, G-box; TATA, TATAbox). Continuous and dotted lines indicate DNA-protein interactions and protein-protein interactions, respectively. A displacement arrow (open line) indicates overexpression of bZIP53 in Pro35S:bZIP53 plants. bZIP53 increased expression in leaves favors heterodimer formation (hd) with group C bZIP10 and bZIP25 and triggers induction of seed gene expression in this organ. 
equilibrium displacement in dimer-monomer concentrations, and (3) heterodimer interactions with other protein factors that increase activation. Here, we showed that the second and third possibilities must be operating in vivo in the regulation of MAT genes. In support of this is the increased activation observed in protoplast systems for the heterodimers, which is independent of their DNA binding when used as GAL4-BD protein fusions (Figure $4 \mathrm{E})$, and their interaction and enhanced gene activation when assayed with $\mathrm{ABI} 3$ (Figure 6). In addition, heterodimer formation seems to protect bZIP proteins from degradation (see Supplemental Figure 3 online) and therefore provides an additional mechanism that contributes to the observed synergistic effect on transcription. Although not investigated in this study, the first possibility might also explain how diversification in target binding sites can lead to variations in the expression of different SSP genes (Conceicao Ada and Krebbers, 1994), depending on the binding properties of the heterodimers prevailing temporally and spatially in the seed. In conclusion, the concurrence of several regulatory mechanisms must be operating on the underlying bZIP network.

\section{bZIP53, Group C bZIPs, and ABI3 Physically and Functionally} Interact in the Control of Seed Maturation Genes

Current models of regulation of MAT gene expression in the seed entail the participation of bZIP and B3 interacting proteins, particularly bZIP10 and 25 and ABI3. We have demonstrated that bZIP53 is a positive factor in this regulation and that its interaction with group $\mathrm{C} b Z \mathrm{IPs}$ results in enhanced activation of the heterodimers. Although yeast and protoplast two-hybrid data indicate that bZIP53 on its own is not able to interact with $A B I 3$, heterodimerizations should not preclude the group $\mathrm{C}$ bZIP-ABI3 interactions. Three-hybrid and BiFC analyses confirmed that bZIP heterodimerization of bZIP10 or bZIP25 with bZIP53 does not interfere with $\mathrm{AB} \mid 3$ interaction and supports the existence of a ternary complex. $\mathrm{ABI} 3$ has been shown to bind to the RY element (Mönke et al., 2004), and a functional interplay between G-box and RY-element in MAT gene regulation has been previously described (Ezcurra et al., 2000; Lara et al., 2003). In this report, we clearly demonstrate functional interplay between bZIP heterodimers and $A B I 3$, presumably by ternary complex formation.

By altering the bZIP expression levels in leaves, we can still see functionality of the bZIP heterodimers despite the absence of the ABI3 protein (Parcy et al., 1994), though their mediated activation occurs at a significantly lower level. Further experiments in bZIP53 overexpressing and knockdown plants show that although bZIP10 and/or ABI3 can enhance MAT gene activation to a certain extent, full-level expression clearly depends on the presence of bZIP53 (see Figure 7B). In this respect, nonseed model systems, such as protoplasts and bombarded leaves, might not fully reflect the native situation in seeds. Nevertheless, all results obtained in these systems are in agreement with the intrinsic reduction of MAT gene expression observed in seeds of mutant bzip53 plants. On the other hand, similar experiments performed in cereal systems that allowed the postulation of regulatory models have been confirmed in null mutants and transgenic plants (Norre et al., 2002). In summary, the output in MAT gene expression depends on all partners, $A B I 3$ and bZIPs, and is quantitatively triggered by the amount of bZIP heterodimers.

Complex formation between the bZIP TF ABI5 and ABI3 have been recognized in LEA but not in MAT gene regulation (Bensmihen et al., 2002). Our findings suggest that the same principle based on $\mathrm{ABI} 3$ protein interactions operates on MAT expression, but inclusion of different bZIP partners defines distinct subsets of target genes. Therefore, this study provides further insight into the synergistic action of TFs and formation of enhanceosomelike protein-DNA complexes, which control seed gene transcription.

A Model of Seed Maturation Gene Expression Triggered by bZIP53 Heterodimerization and Protein Complex Formation

Based on previous findings and data from this study, the working model in Figure 8 is proposed. The function of the previously described group C bZIP factors bZIP10 and bZIP25 in seed maturation strongly depends on bZIP53 as a heterodimerization partner. Spatial and developmentally controlled expression of bZIP53 is precisely timed, leading to increased amounts in the seed from mid maturation. Subsequent accumulation of bZIP53 would trigger heterodimer formation with bZIP10 or bZIP25 as prerequisite for efficient promoter binding and target SSP gene activation. $A B \mid 3$ protein, which also accumulates during seed maturation, interacts with the bZIP heterodimer via bZIP10 or bZIP25 and provides an additional increment in promoter activation. Hence, SSP gene regulation is driven by the interplay of (at least) two maturation-dependent transcriptional regulators, namely, bZIP53 and ABI3. The crucial role of $A B \mid 3$ in this process was largely uncovered through the characterization of abi3 lossof-function mutants (Parcy et al., 1994). This factor has a prominent role in seed maturation and germination gene expression programs. In this respect, its participation in a ternary complex with C/S bZIPs might partly explain many of numerous pleotropic effects observed in abi3 mutants. Interestingly, transcriptomic data suggest that bZIP53 and bZIP10 expression levels are reduced in mutant abi3-4 developing seeds (Carrera et al., 2008).

In vegetative tissues, where bZIP10 and bZIP25 are constitutively present at low levels, the ectopic high-level expression of ABI3 can lead to the activation of SSP genes (Parcy et al., 1994; Parcy and Giraudat, 1997). Likewise, ectopic high-level expression of bZIP53 can substitute for the presence of $A B \mid 3$, triggering SSP gene expression even more effectively than this factor. In summary, we propose that bZIP53 acts as a key regulator, which drives a subset of MAT genes mainly by the formation of heterodimers with a high activation capacity. Although further research is needed to elucidate distinct interacting partners and target genes both in vegetative and seed tissues, bZIP53 might represent an important target for manipulating seed storage compound accumulation and establishment of desiccation tolerance.

\section{METHODS}

\section{Plant Materials and Transformation}

For plant and protoplast transformation, particle bombardment, and ChIP experiments, Arabidopsis thaliana ecotype Col-0 was grown on soil or 
Murashige and Skoog plates under controlled environmental conditions at 16 -h-light/8-h-dark cycles. To improve germination uniformity, seeds were pretreated at $4^{\circ} \mathrm{C}$ for 2 to $4 \mathrm{~d}$. Floral dip transformations were performed using the Agrobacterium tumefaciens strain GV3101 (Weigel and Glazebrook, 2002). Pro35S:bZIP53, Pro35S:HA-bZIP53, ProbZIP53: GUS, and bzip53 (NASC ID: N569883) plants were described by Weltmeier et al. (2006).

\section{In Situ Hybridization and Histochemistry}

In situ hybridization was performed according to Ferrandiz et al. (2000). Forward 5 '-TTGTCCAATGCAACCCAATCA-3' and reverse 5'-ACAAGACTAGAGGACTGAGGCT-3' bZIP53-specific oligonucleotides were used to amplify a 200-bp fragment from the 3 ' untranslated region. The fragment was inserted into pGEM-T easy (Promega), and the sense and antisense probes were generated as specified by Lara et al. (2003). The construction of ProbZIP53:GUS plants has been described by Weltmeier et al. (2006). GUS staining was performed according to Stangeland and Salehian (2002) with some modifications. The siliques were incubated in GUS assay buffer overnight at room temperature under vacuum and transferred to an ethanol/acetic acid (1:1) solution for $4 \mathrm{~h}$ (young seeds) or $12 \mathrm{~h}$ (mature seeds). Clearing was performed by incubating tissues in Hoyer's light medium ( $100 \mathrm{~g}$ chloral hydrate in $60 \mathrm{~mL}$ water) for $12 \mathrm{~h}$ (young seeds) or $24 \mathrm{~h}$ (mature seeds) at room temperature. Samples were analyzed with a Zeiss Axiophot microscope.

\section{qRT-PCR}

RNA was isolated from seedlings and siliques as described by OñateSánchez and Vicente-Carbajosa (2008). cDNA synthesis was performed according to Oñate-Sánchez and Singh (2002), and between 8 and $16 \mathrm{ng}$ were used as a template for $\mathrm{QRT}$-PCR together with forward and reverse oligonucleotides $(0.5 \mu \mathrm{M}$ each) in $1 \times$ Power SYBR Green PCR Master mix (Applied Biosystems). Cycling conditions (ABI Prism 7300; Applied Biosystems) were as follows: 10 min at $95^{\circ} \mathrm{C}, 50$ cycles of $15 \mathrm{~s}$ at $95^{\circ} \mathrm{C}$, and 60 $\mathrm{s}$ at $60^{\circ} \mathrm{C}$, linked to a default dissociation stage program to detect nonspecific amplification. Primers are provided in Supplemental Table 1 online. At least two technical and two biological replicates were analyzed in all the experiments

\section{Transient Expression Assays and Constructs}

Protoplast isolation, transformation, construction of effector plasmids, and immunoblot analysis were performed according to Ehlert et al. (2006) and Weltmeier et al. (2006). For reporter gene assays, $9 \mu \mathrm{g}$ reporter and $14 \mu \mathrm{g}$ of each effector were used if not stated otherwise. Three micrograms of Pro35S:NAN plasmid was added for normalization (Kirby and Kavanagh, 2002). For three-hybrid analysis, $9 \mu \mathrm{g}$ of each reporter, BD-, AD-, and HA-plasmid were applied. The overall amount of DNA was set to $40 \mu \mathrm{g}$ by adding pUC19 plasmid DNA. Particle bombardment was performed according to Lara et al. (2003). Data presented for the different assayed constructs are derived from three independent experiments, including six plates with 10 leaves per plate. Pro2S2:GUS reporter vector is a pUC19-derived plasmid containing the GUS reporter gene under the control of the $2 S 2$ promoter and fused to the 3 '-terminator of the nopaline synthase gene ( $3^{\prime}$-nos) (Lara et al., 2003). Effector plasmids for the expression of bZIP53, bZIP10, bZIP25, and ABI3 were generated by cloning the corresponding coding sequence under the control of the Pro35S and a 3'-nos terminator (Lara et al., 2003) in the pJIT60 plasmid (Guerineau, 1995). Three micrograms of Pro35S:NAN plasmid was added for normalization. GUS and NAN enzyme assays were performed according to Kirby and Kavanagh (2002). Immunoblot analysis was performed according to Weltmeier et al. (2006) using $\alpha-\mathrm{HA}$ and $\alpha-\mathrm{BD}$ antisera (SantaCruz).

\section{ChIP}

ChIP was performed as described by Weltmeier et al. (2006) using an HAspecific antibody (SantaCruz). 2 S2 primers were used to amplify a 306-bp fragment of the $2 S 2$ promoter ( $5^{\prime}$-GACCGGTGACCTGCGTGTA-3' and 5'-GACTTGCATGGAGTTCACGTG-3'). The difference between the resulting $C(t)$ values of wild-type and Pro35S:HA-bZIP53 overexpressor was calculated and normalized with the input controls of these samples that were analyzed with the same primers. For normalization, PCR was performed with unspecific actin (ACT7) promoter primers (5'-CGTTTCGCTTTCCTTAGTGTTAGCT-3' and 5'-AGCGAACGGATCTAGAGACTCACCTTG-3').

\section{ELISA-Based Protein-DNA Binding Assays}

The cDNAs encoding bZIP10, bZIP25, bZIP53, and ABI3 proteins were cloned into the expression vectors pET23a (Novagen) to generate T7-epitope tagged proteins (Lara et al., 2003). Expression in Escherichia coli and preparation of protein extracts were performed as previously described (Lara et al., 2003). Biotinylated complementary oligonucleotides derived from the At2S2 promoter containing a G-box sequence ( 5 '-CATGCAATGCATTCTTACACGTGATTGCATGCAAA- 3 ' and $5^{\prime}$-TाTGCATGCAATCACGTGTAAGAATGCATTGCATG- $3^{\prime}$ ) or a mutated version (5'-CATGCAATGCATTCTTACAaGgGATTGCATGCAAA-3' and 5'-TTTGCATGCAATCCCITgTAAGAATGCATTGCATG-3') were annealed in TES buffer ( $10 \mathrm{mM}$ Tris, $\mathrm{pH} 8,1 \mathrm{mM}$ EDTA, and $300 \mathrm{mM} \mathrm{NaCl})$. The appropriate double-stranded DNA (2 pmoles; target) in $60 \mu \mathrm{L}$ of TBS-T buffer ( $20 \mathrm{mM}$ Tris, $\mathrm{pH} 7.4,180 \mathrm{mM} \mathrm{NaCl}$, and 0.1\% Tween 20) was added to each well of a streptavidin-coated plate (Nunc Inmobilizer) and incubated $1 \mathrm{~h}$ at $37^{\circ} \mathrm{C}$, and nonbound targets were removed by washing three times with TBS-T. Wells were blocked by incubating $30 \mathrm{~min}$ at room temperature and gentle shaking (150 rpm) with $60 \mu \mathrm{L}$ of $1 \%$ blocking agent (Roche) in TBS-T and washed three times with TBS-T. Twenty microliters of the protein extracts were mixed with $40 \mu \mathrm{L}$ of binding buffer ( 4 mM HEPES, pH 7.5, $100 \mathrm{mM} \mathrm{KCl}, 0.2 \%$ BSA, $8 \%$ glycerol, and $5 \mathrm{mM}$ DTT) in microfuge tubes and incubated $15 \mathrm{~min}$ on ice. The mixes were added to the wells of the plate, the binding reactions incubated for $1 \mathrm{~h}$ at room temperature with gentle shaking, and well washed three times with TBS-T. For epitope detection, we added $60 \mu \mathrm{L}$ of a 1:5000 dilution of the antibody ( $\mathrm{T} 7 \mathrm{tag}$ horseradish peroxidase conjugate; Novagen) in TBS-T to each well. Following incubation for $1 \mathrm{~h}$ at room temperature at $150 \mathrm{rpm}$, the wells were four times washed with TBS-T. The antibody peroxidaseconjugated reaction was performed according to manufacturer's instructions, and the activity was measured at $492 \mathrm{~nm}$ using an ELISA plate reader (Tecan) with filter setting at 650 for reference.

\section{Yeast Two- and Three-Hybrid Analyses}

Yeast two-hybrid analyses were done as described previously (Lara et al., 2003). BD and AD constructs were generated using appropriate restriction enzymes and cloning the indicated open reading frames into the pGBT9 and pGAD424 plasmids (Clontech), respectively, as follows: EcoRI/Sall sites for the BD-bZIP53, AD-bZIP53, BD-ABI5, and AD-ABI5 constructs and BamHI/Sall sites for the BD-ABI3 and AD-ABI3 constructs. BD-bZIP10, AD-bZIP10, BD-bZIP25, and AD-bZIP25 were described by Lara et al. (2003). To analyze ternary complex formation in yeast, we used $B D$ and $A D$ constructs from the two-hybrid assays and expressed a third protein whose coding sequences were inserted into the EcoRI/Sall sites of the pTFT1 plasmid (Egea-Cortines et al., 1999).

\section{BiFC}

Experimental procedures were according to Moreno-Risueno et al. (2007). In general, at least two independent assays were performed that 
included four independent plates bombarded for every individual construct or the different combinations. Comparisons were based on transformation efficiencies estimated to Pro35S: green fluorescent protein as a reference. Vectors used have been described by Weltmeier et al. (2006).

\section{Accession Numbers}

Arabidopsis Genome Initiative identifiers for the genes mentioned in this article are as follows: bZIP53 (At3g62420), bZIP10 (At4g02640), bZIP25 (At3g54620), bZIP1 (At5g49450), 2S2 (At4g27150), CRU3 (At4g28520), LEA76 (At3g15670), ProDH (At5g38710), UBI (At5g25760), ACT7 (At5g09810), AB/3 (At3g24650), ABI5 (At2g36270), ASN1 (At3g47340), CRA1 (At5g44120), and At HSD1 (At5g50600).

\section{Supplemental Data}

The following materials are available in the online version of this article.

Supplemental Figure 1. Analysis of Putative bZIP53 Target Genes in Plants Constitutively Expressing HA-bZIP53 or bZIP53.

Suplemental Figure 2. Transient Expression of bZIP53 in Combination with bZIP10 or bZIP25 Synergistically Enhances Transactivation of CRU3 and 2S2 Promoters.

Supplemental Figure 3. Analysis of Effector Proteins Transiently Expressed in Arabidopsis Protoplasts.

Supplemental Figure 4. Ternary Protein Interaction Studied in a Yeast Three-Hybrid System.

Supplemental Figure 5. Transient Expression by Microparticle Bombardment of Arabidopsis Leaves from Columbia Wild Type and Plants with Increased (Pro35S:bZIP53) or Decreased (bzip53) Expression of bZIP53.

Supplemental Table 1. Primers Used in This Study.

\section{ACKNOWLEDGMENTS}

We thank Mar Gonzalez and Anna Herman for their valuable technica assistance and Caroline Carsjens and Louise Thatcher for proofreading. Critical comments and suggestions from anonymous referees and the Scientific Editor greatly improved previous versions of this study. Financial support from the Ministerio de Educación y Ciencia, Spain (GEN2003-20859 and BIO2007-68073) is acknowledged. R.A. is the recipient of a predoctoral fellowship from the Spanish Agency for International Cooperation. L.O.-S. was supported by the Spanish Ministry for Education and Science with a Ramón and Cajal Contract and funded with a Marie Curie International Reintegration Grant (036524). W.D.-L. is supported by Deutsche Forschungsgemeinschaft and Deurscher Akademische Auslausik Dienst.

Received September 2 2008; revised May 6, 2009; accepted May 26, 2009; published June 16, 2009.

\section{REFERENCES}

Albani, D., Hammond Kosack, M.C., Smith, C., Conlan, S., Colot, V., Holdsworth, M., and Bevan, M.W. (1997). The wheat transcriptional activator SPA: A seed-specific bZIP protein that recognizes the GCN4-like motif in the bifactorial endosperm box of prolamin genes. Plant Cell 9: 171-184.

Baena-Gonzalez, E., Rolland, F., Thevelein, J.M., and Sheen, J.
(2007). A central integrator of transcription networks in plant stress and energy signalling. Nature 448: 938-942.

Bensmihen, S., Rippa, S., Lambert, G., Jublot, D., Pautot, V., Granier, F., Giraudat, J., and Parcy, F. (2002). The homologous $\mathrm{ABI5}$ and EEL transcription factors function antagonistically to finetune gene expression during late embryogenesis. Plant Cell 14: 13911403.

Bies-Etheve, N., Gaubier-Comella, P., Debures, A., Lasserre, E., Jobet, E., Raynal, M., Cooke, R., and Delseny, M. (2008). Inventory, evolution and expression profiling diversity of the LEA (late embryogenesis abundant) protein gene family in Arabidopsis thaliana. Plant Mol. Biol. 67: 107-124.

Bowman, J. (1994). Arabidopsis: An Atlas of Morphology and Development. (New York: Springer-Verlag).

Braybrook, S.A., Stone, S.L., Park, S., Bui, A.Q., Le, B.H., Fischer, R.L., Goldberg, R.B., and Harada, J.J. (2006). Genes directly regulated by LEAFY COTYLEDON2 provide insight into the control of embryo maturation and somatic embryogenesis. Proc. Natl. Acad. Sci. USA 103: 3468-3473.

Carrera, E., Holman, T., Medhurst, A., Dietrich, D., Footitt, S., Theodoulou, F.L., and Holdsworth, M.J. (2008). Seed after-ripening is a discrete developmental pathway associated with specific gene networks in Arabidopsis. Plant J. 53: 214-224.

Conceicao Ada, S., and Krebbers, E. (1994). A cotyledon regulatory region is responsible for the different spatial expression patterns of Arabidopsis 2S albumin genes. Plant J. 5: 493-505.

Egea-Cortines, M., Saedler, H., and Sommer, H. (1999). Ternary complex formation between the MADS-box proteins SQUAMOSA, DEFICIENS and GLOBOSA is involved in the control of architecture in Antirninum majus. EMBO J. 18: 5370-5379.

Ehlert, A., Weltmeier, F., Wang, X., Mayer, C.S., Smeekens, S., Vicente-Carbajosa, J., and Dröge-Laser, W. (2006). Two-hybrid protein-protein interaction analysis in Arabidopsis protoplasts: Establishment of a heterodimerisation map of group-C and S bZIP transcription factors. Plant J. 46: 890-900.

Ellenberger, T.E., Brandl, C.J., Struhl, K., and Harrison, S.C. (1992). The GCN4 basic region leucine zipper binds DNA as a dimer of uninterrupted alpha helices: Crystal structure of the protein-DNA complex. Cell 71: 1223-1237.

Ezcurra, I., Wycliffe, P., Nehlin, L., Ellerstrom, M., and Rask, L. (2000). Transactivation of the Brassica napus napin promoter by $\mathrm{ABI} 3$ requires interaction of the conserved $\mathrm{B} 2$ and $\mathrm{B} 3$ domains of $\mathrm{AB} / 3$ with different cis-elements: B2 mediates activation through an ABRE, whereas B3 interacts with an RY/G-box. Plant J. 24: 57-66.

Ferrandiz, C., Gu, Q., Martienssen, R., and Yanofsky, M.F. (2000). Redundant regulation of meristem identity and plant architecture by FRUITFULL, APETALA1 and CAULIFLOWER. Development 127: 725-734.

Finkelstein, R.R., and Lynch, T.J. (2000). The Arabidopsis abscisic acid response gene $A B I 5$ encodes a basic leucine zipper transcription factor. Plant Cell 12: 599-609.

Giraudat, J., Hauge, B.M., Valon, C., Smalle, J., Parcy, F., and Goodman, H.M. (1992). Isolation of the Arabidopsis ABI3 gene by positional cloning. Plant Cell 4: 1251-1261.

Gruis, D., Schulze, J., and Jung, R. (2004). Storage protein accumulation in the absence of the vacuolar processing enzyme family of cysteine proteases. Plant Cell 16: 270-290.

Guerineau, F. (1995). Tools for expressing foreign genes in plants. Methods Mol. Biol. 49: 1-32.

Gutierrez, L., Van Wuytswinkel, O., Castelain, M., and Bellini, C. (2007). Combined networks regulating seed maturation. Trends Plant Sci. 12: 294-300.

Hanson, J., Hanssen, M., Wiese, A., Hendriks, M.M.W.B., and 
Smeekens, S. (2008). The sucrose regulated transcription factor bZIP11 affects amino acid metabolism by regulating the expression of Asparagin Synthase1 and Proline Dehydrogenase 2. Plant J. 53: 935-949.

Hartings, H., Maddaloni, M., Lazzaroni, N., Di Fonzo, N., Motto, M., Salamini, F., and Thompson, R. (1989). The O2 gene which regulates zein deposition in maize endosperm encodes a protein with structural homologies to transcriptional activators. EMBO J. 8: 2795-2801.

Hundertmark, M., and Hincha, D.K. (2008). LEA (late embryogenesis abundant) proteins and their encoding genes in Arabidopsis thaliana. BMC Genomics 9: 1-18.

Jakoby, M., Weisshaar, B., Dröge-Laser, W., Vicente-Carbajosa, J., Tiedemann, J., Kroj, T., and Parcy, F. (2002). bZIP transcription factors in Arabidopsis. Trends Plant Sci. 7: 106-111.

Kaminaka, H., Nake, C., Epple, P., Dittgen, J., Schütze, K., Chaban, C., Holt III, B.F., Merkle, T., Schafer, E., Harter, K., and Dangl, J.L. (2006). bZIP10-LSD1 antagonism modulates basal defense and cell death in Arabidopsis following infection. EMBO J. 25: 4400-4411.

Kirby, J., and Kavanagh, T.A. (2002). NAN fusions: A synthetic sialidase reporter gene as a sensitive and versatile partner for GUS. Plant J. 32: $391-400$

Kroj, T., Savino, G., Valon, C., Giraudat, J., and Parcy, F. (2003). Regulation of storage protein gene expression in Arabidopsis. Development 130: 6065-6073.

Lam, H.M., Wong, P., Chan, H.K., Yam, K.M., Chen, L., Chow, C.M., and Coruzzi, G.M. (2003). Overexpression of the ASN1 gene enhances nitrogen status in seeds of Arabidopsis. Plant Physiol. 132: 926-935.

Lara, P., Oñate-Sánchez, L., Abraham, Z., Ferrandiz, C., Diaz, I., Carbonero, P., and Vicente-Carbajosa, J. (2003). Synergistic activation of seed storage protein gene expression in Arabidopsis by $\mathrm{ABI} 3$ and two bZIPs related to OPAQUE2. J. Biol. Chem. 278: 2100321011.

Li, F., Asami, T., Wu, X., Tsang, E.W., and Cutler, A.J. (2007). A putative hydroxysteroid dehydrogenase involved in regulating plant growth and development. Plant Physiol. 145: 87-97.

Mönke, G., Altschmied, L., Tewes, A., Reidt, W., Mock, H.P., Bäumlein, H., and Conrad, U. (2004). Seed-specific transcription factors $A B I 3$ and FUS3: Molecular interaction with DNA. Planta 219: 158-166.

Moreno-Risueno, M.A., Diaz, I., Carrillo, L., Fuentes, R., and Carbonero, P. (2007). The HvDOF19 transcription factor mediates the abscisic acid-dependent repression of hydrolase genes in germinating barley aleurone. Plant J. 51: 352-365.

Nakamura, S., Lynch, T.J., and Finkelstein, R.R. (2001). Physical interactions between ABA response loci of Arabidopsis. Plant J. 26: 627-635.

Nakashima, K., Fujita, Y., Katsura, K., Maruyama, K., Narusaka, Y., Seki, M., Shinozaki, K., and Yamaguchi-Shinozaki, K. (2006). Transcriptional regulation of $\mathrm{ABI}$ - and $\mathrm{ABA}$-responsive genes including RD29B and RD29A in seeds, germinating embryos, and seedlings of Arabidopsis. Plant Mol. Biol. 60: 51-68.

Nantel, A., and Quatrano, R.S. (1996). Characterization of three rice basic/leucine zipper factors, including two inhibitors of EmBP-1 DNA binding activity. J. Biol. Chem. 271: 31296-31305.

Norre, F., Peyrot, C., Garcia, C., Rance, I., Drevet, J., Theisen, M., and Gruber, V. (2002). Powerful effect of an atypical bifactorial endosperm box from wheat HMWG-Dx5 promoter in maize endosperm. Plant Mol. Biol. 50: 699-712.

Oñate, L., Vicente Carbajosa, J., Lara, P., Diaz, I., and Carbonero, P. (1999). Barley BLZ2, a seed-specific bZIP protein that interacts with $\mathrm{BLZ1}$ in vivo and activates transcription from the GCN4-like motif of
B-hordein promoters in barley endosperm. J. Biol. Chem. 274: 91759182.

Oñate-Sánchez, L., and Singh, K.B. (2002). Identification of Arabidopsis ethylene-responsive element binding factors with distinct induction kinetics after pathogen infection. Plant Physiol. 128: 1313-1322. Oñate-Sánchez, L., and Vicente-Carbajosa, J. (2008). DNA-free RNA isolation protocols for Arabidopsis thaliana, including seeds and siliques. BMC Res Notes. 1: 93

Parcy, F., and Giraudat, J. (1997). Interactions between the ABI1 and the ectopically expressed $A B I 3$ genes in controlling abscisic acid responses in Arabidopsis vegetative tissues. Plant J. 11: 693-702.

Parcy, F., Valon, C., Raynal, M., Gaubier-Comella, P., Delseny, M., and Giraudat, J. (1994). Regulation of gene expression programs during Arabidopsis seed development: Roles of the ABI3 locus and of endogenous abscisic acid. Plant Cell 6: 1567-1582

Rook, F., Gerrits, N., Kortstee, A., van Kampen, M., Borrias, M., Weisbeek, P., and Smeekens, S. (1998). Sucrose-specific signalling represses translation of the Arabidopsis ATB2 bZIP transcription factor gene. Plant J. 15: 253-263.

Santos-Mendoza, M., Dubreucq, B., Baud, S., Parcy, F., Caboche, M., and Lepiniec, L. (2008). Deciphering gene regulatory networks that control seed development and maturation in Arabidopsis. Plant J. 54: 608-620.

Satoh, R., Fujita, Y., Nakashima, K., Shinozaki, K., and YamaguchiShinozaki, K. (2004). A novel subgroup of bZIP proteins functions as transcriptional activators in hypoosmolarity-responsive expression of the ProDH gene in Arabidopsis. Plant Cell Physiol. 45: 309-317.

Schallau, A., Kakhovskaya, I., Tewes, A., Czihal, A., Tiedemann, J., Mohr, M., Grosse, I., Manteuffel, R., and Bäumlein, H. (2008). Phylogenetic footprints in fern spore- and seed-specific gene promoters. Plant J. 53: 414-424.

Schmidt, R.J., Burr, F.A., Aukerman, M.J., and Burr, B. (1990). Maize regulatory gene opaque-2 encodes a protein with a "leucine-zipper" motif that binds to zein DNA. Proc. Natl. Acad. Sci. USA 87: 46-50.

Stangeland, B., and Salehian, Z. (2002). An improved clearing method for GUS assay in Arabidopsis endosperm and seeds. Plant Mol. Biol. Rep. 20: 107-114.

To, A., Valon, C., Savino, G., Guilleminot, J., Devic, M., Giraudat, J., and Parcy, F. (2006). A network of local and redundant gene regulation governs Arabidopsis seed maturation. Plant Cell 18: 1642-1651.

Tunnacliffe, A., and Wise, M.J. (2007). The continuing conundrum of the LEA proteins. Naturwissenschaften 94: 791-812.

Usadel, B., Blasing, O.E., Gibon, Y., Retzlaff, K., Hohne, M. Gunther, M., and Stitt, M. (2008). Global transcript levels respond to small changes of the carbon status during progressive exhaustion of carbohydrates in Arabidopsis rosettes. Plant Physiol. 146: 1834-1861.

Vicente-Carbajosa, J., and Carbonero, P. (2005). Seed maturation: Developing an intrusive phase to accomplish a quiescent state. Int. J. Dev. Biol. 49: 645-651.

Walter, M., Chaban, C., Schütze, K., Batistic, O., Weckermann, K. Nake, C., Blazevic, D., Grefen, C., Schumacher, K., Oecking, C., Harter, K., and Kudla, J. (2004). Visualization of protein interactions in living plant cells using bimolecular fluorescence complementation. Plant J. 40: 428-438.

Wang, H.W., Zhang, B., Hao, Y.J., Huang, J., Tian, A.G., Liao, Y. Zhang, J.S., and Chen, S.Y. (2007). The soybean Dof-type transcription factor genes, GmDof4 and GmDof11, enhance lipid content in the seeds of transgenic Arabidopsis plants. Plant J. 52: 716-729.

Weber, H., Borisjuk, L., and Wobus, U. (2005). Molecular physiology of legume seed development. Annu. Rev. Plant Biol. 56: 253-279.

Weigel, R., and Glazebrook, J. (2002). Arabidopsis: A Laboraton Manual. (Cold Spring Harbor, NY: Cold Spring Harbor Laboratory Press). 
Weltmeier, F., Ehlert, A., Mayer, C.S., Dietrich, K., Wang, X. Schütze, K., Harter, K., Vicente-Carbajosa, J., and Dröge-Laser, W. (2006). Combinatorial control of Arabidopsis proline dehydrogenase transcription by specific heterodimerisation bZIP transcription factors. EMBO J. 25: 3133-3143.

Weltmeier, F., Rahmani, F., Ehlert, A., Dietrich, K., Schütze, K. Wang, X., Chaban, C., Hanson, J., Teige, M., Harter, K., VicenteCarbajosa, J., Smeekens, S., and Dröge-Laser, W. (2009). Expres- sion patterns within the Arabidopsis $\mathrm{C} / \mathrm{S} 1 \mathrm{bZIP}$ transcription factor network: Availability of heterodimerization partners controls gene expression during stress response and development. Plant Mol. Biol. 69: 107-119.

Xu, D., Duan, X., Wang, B., Hong, B., Ho, T., and Wu, R. (1996) Expression of a late embryogenesis abundant protein gene, HVA1, from barley confers tolerance to water deficit and salt stress in transgenic rice. Plant Physiol. 110: 249-257. 


\title{
8 Genome-wide analysis reveals the impact of bZIP heterodimers on target promoter selection
}

\author{
Gene expression analysis reveals differential functions of bZIP dimers in \\ protoplasts
}

\author{
Micha Hanssen, Andrea Ehlert ${ }^{1}$, Sjef Smeekens, Wolfgang Dröge-Laser ${ }^{1}$ and Johannes Hanson
}

Molecular Plant Physiology, Utrecht University, Padualaan 8, 3584 CH Utrecht, The Netherlands

Albrecht-von-Haller-Institut, Universität Göttingen, Untere Karspüle 2, D-37073 Göttingen, Germany

\begin{abstract}
Basic region/leucine zipper motif (bZIP) proteins comprise a large family of plant transcription factors. bZIP transcription factors affect gene expression by binding DNA as specific homo- or heterodimeric complexes. The specific transcriptional activity of different related dimer species has not been studied in great detail in plants. Unraveling the functions of the complex network of different bZIP dimers in plants demands determination of the target genes of each dimer species. For this purpose, Arabidopsis thaliana mesophyll protoplasts were transfected with plasmid DNA encoding two different bZIP transcription factors: bZIP10 (At4g02640) and bZIP11 (At4g34590). High bZIP10 protein levels hardly affected gene expression, as revealed by global gene expression analysis. Increased expression of bZIP11 strongly affected gene expression, but when bZIP11 was transfected together with bZIP10 a major synergistic effect on gene expression was observed. These results provide solid evidence that different bZIP dimers have differential effects on gene expression.
\end{abstract}

\section{Introduction}

Basic region/leucine zipper motif (bZIP) proteins form a large family of transcription factors defined by a basic region that interacts in a sequence specific manner with the major groove of DNA through hydrogen bonding, and an amphipatic leucine zipper region that is responsible for dimerization. The Arabidopsis genome holds a total of 75 bZIP genes, only a handful of which have been functionally described in the literature. Jakoby et. al. (Jakoby et al., 2002) divided all bZIPs in Arabidopsis into classes, based on shared domains in their sequence. One particular class, the S-class consists of low molecular weight proteins that lack other known domains but harbour an unusually long zipper domain of 8-9 leucines. An S-subclass (S1) of bZIPs is characterised by the presence of a highly conserved uORF in the 5 ' leader of its messenger RNA that allows translational control by sucrose (Rook et al., 1998c; Wiese et al., 2004; Weltmeier et al., 2009). The best-studied member of this class, bZIP11, is involved in the regulation of amino acid metabolism, by affecting the expression of ASN1 and ProDH2 (Hanson et al., 2008).

In order to bind DNA and affect gene expression, bZIP transcription factors must form dimers. S1-class bZIPs preferably dimerise with members of the C-class, although it has been shown that S1-class bZIPs, in particular bZIP11, are also able to form homodimers (Ehlert et al., 2006a). C-class bZIPs in contrast, do not tend to form homodimers. The formation of bZIP homoand heterodimers potentially offers tremendous combinatorial flexibility to regulate gene transcription. For instance, ProDH gene expression is known to be regulated by several 
heterodimeric complexes consisting of a $\mathrm{C}$ class and an S1-class bZIP protein (Satoh et al., 2004; Weltmeier et al., 2006). Other genes however, seem to be regulated by specific bZIP proteins, as was determined by micro-array analysis (Hanson et al., 2008). So far, the possibility of different gene-regulatory functions of different dimers, consisting of related bZIP proteins has not been investigated. For this purpose, 5'HAtagged bZIP10 and 5'HA-tagged bZIP11 enconding plasmids were transfected into Arabidopsis thaliana mesophyll protoplasts, either alone or together. Overexpression of only bZIP11 would result in effects on gene expression by mostly homodimers, even though effects of heterodimers involving endogenously present bZIPs cannot be excluded. Changes in gene expression after overexpression of both bZIP10 and bZIP11 in the same protoplasts likely represent bZIP10+11 dimer effetcts. In this manuscript we demonstrate the differential gene-regulatory activity of different bZIP dimers, and thereby show the importance of dimerization specificity in the regulation of gene expression by bZIP transcription factors.

\section{Results}

\section{mRNA profiling of mesophyll protoplasts is a suitable system for gene expression analysis}

Gene expression in Arabidopsis mesophyll protoplasts, transiently overexpressing bZIPs was studied to identify the targets of bZIP transcription factors. Overexpression of bZIP genes was achieved by transfecting Arabidopsis mesophyll protoplasts with, plasmids encoding 5'HA-tagged bZIP10 or bZIP11, combinations thereof or an empty vector as control. Sixteen hours following transfection, the mRNA expression levels of bZIP10 and bZIP11 were 120 and 400-fold higher, respectively, than levels in protoplasts transfected with the empty vector. The bZIP10 and bZIP11 proteins were demonstrated to be abundantly expressed and fully translated by Western analysis, using antibodies directed against the HA-tag (Figure 1). After transfection, global gene expression in protoplasts was compared using the ATH1 GeneChip $^{\circledR}$. Next, the expression for each probeset was calculated, a linear model was fit to the data and expression levels of genes were compared (Figure 2). Results obtained from the array data were confirmed by quantitative realtime PCR (Table 1). In all protoplast preparations, a representative set of about 9000 genes was expressed to levels statistically significant above background. Previous experiments have shown around 11000 genes to be expressed in 7-day-old seedlings

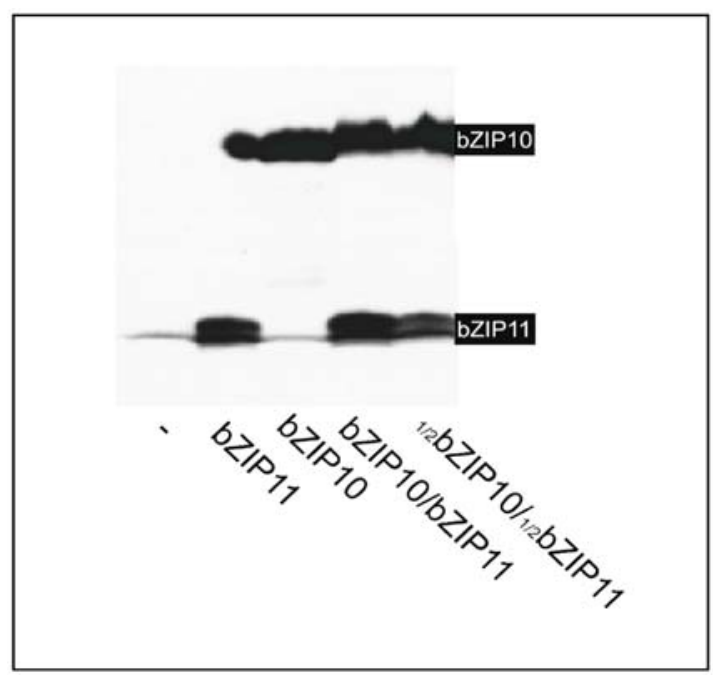

Figure 1 bZIPs are highly expressed in transfected protoplasts

Western analysis after immunoprecipitation with an HA antibody shows that the full- length bZIP fusion proteins are highly abundant in transfected protoplasts. Protoplasts were transfected with an empty control vector (-), HA- tagged 35S: bZIP11 or both bZIP10 and bZIP11 fusion constructs. In the right- most lane results are shown for an experiment where both bZIP fusion constructs were transfected at half the concentration of the plasmids. The boxes right of the blot represent the mobility of bZIP10 and bZIP11 HA- fusion proteins as determined by previous experiments and molecular weight markers.

(Allemeersch et al., 2005; Hanson et al., 2008). A MAPMAN representation of the 9000 genes expressed in protoplasts shows that genes of all GO terms in the map are well represented (Figure 3). Inherent to the protoplast system some classes are underrepresented because of tissue specificity. Root or cell wall specific genes are underepresented in mesophyll protoplasts. Nonetheless, these findings indicate that the protoplast system is representative of the whole-plant system and suitable for gene expression studies, as demonstrated before (Birnbaum et al., 2005; Baena-Gonzalez et al., 2007).

\section{Effects of bZIP11 overexpression in seedlings can be reproduced in protoplasts}

A total of 383 genes were expressed at statistically significantly $(\mathrm{q} \leq 0.02)$ higher levels (two-fold) after protoplasts were transfected with plasmid DNA encoding constitutively expressed HA-tagged bZIP11. In an earlier mRNA profiling experiment the effect of 

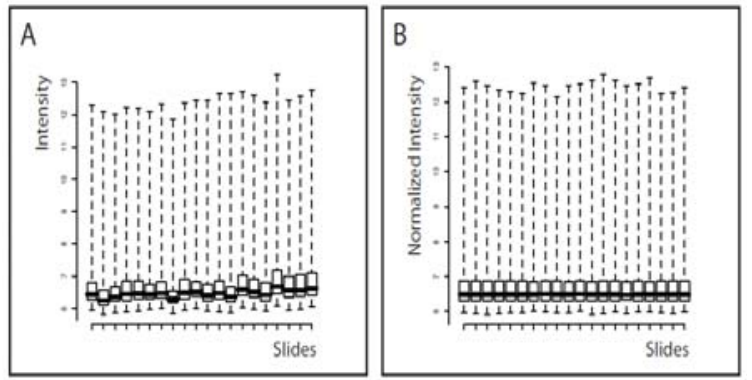

Figure 2. Gene expression in transfected protoplasts

The boxplots show average signal intensities over the slides used before (A) and after (B) normalization. The interquartile ranges are depicted as boxes, the dashed lines represent the $95 \%$ confidence interval and horizontal bars represent median levels.

Table 1. Gene expression levels determined by micro-array are comparable to corresponding levels determined by quantitative real-time PCR

\begin{tabular}{|c|c|c|c|c|c|c|c|c|}
\hline \multirow[b]{2}{*}{ AGI } & \multirow[b]{2}{*}{ Gene name } & \multirow[b]{2}{*}{ Expr level ${ }^{2}$} & \multicolumn{2}{|c|}{ bZIP10 } & \multicolumn{2}{|c|}{ bZIP11 } & \multicolumn{2}{|c|}{ bZIP10 and bZIP11 } \\
\hline & & & Array & Q.PCR & Array & Q.PCR & Array & Q.PCR \\
\hline AT3G47340 & ASN1 & 4.4 & 0.0 & -0.2 & 1.3 & 1.3 & 4.3 & 3.0 \\
\hline AT5G38710 & ProDH2 & 10.0 & -0.3 & -0.4 & 0.4 & 0.6 & 1.0 & 0.9 \\
\hline AT1G10070 & ATBCAT-2 & 4.7 & -0.2 & 0.0 & 1.0 & 1.6 & 3.4 & 3.6 \\
\hline AT4G34590 & bZIP11 & 8.6 & -0.1 & -0.3 & 8.7 & 13.3 & 8.7 & 13.0 \\
\hline AT4G33700 & & 7.4 & 0.3 & -0.2 & 4.3 & 4.5 & 4.4 & 4.0 \\
\hline AT3G30775 & ProDH & 9.5 & 0.5 & 0.5 & 3.2 & 4.1 & 3.6 & 4.3 \\
\hline AT3G18780 & ACT2 & 1.0 & -0.1 & -0.2 & 0.0 & 0.1 & 2.0 & 1.4 \\
\hline AT1G15040 & & 9.4 & 0.2 & 0.0 & 4.3 & 4.3 & 4.9 & 4.6 \\
\hline AT4G35770 & SEN1 & 10.1 & -0.1 & -0.3 & 1.5 & 2.1 & 2.8 & 3.2 \\
\hline
\end{tabular}

elevated levels of bZIP11 on the transcriptome in seedlings was studied (Hanson et al., 2008).

It was found that bZIP11 is a potent activator of gene expression. Upon increasing nuclear bZIP11 levels, a total of 163 genes were expressed at statistically significantly higher levels. Out of these 163 genes, 44 were up regulated in protoplasts overexpressing bZIP11, compared to expression levels in protoplasts transfected with the empty vector (Table 2). Given the set of 11000 expressed genes in the previous study and assuming complete randomness, the binominal probability of picking up 44 out of the 163 up regulated genes from that study would be $2.6 \times 10^{-25}$. This confirms that the experimental system used here is of biological relevance. Interestingly, among the up regulated genes several bZIP transcription factors were identified. The C-class bZIP transcription factor bZIP25 was down regulated more than 3 fold, which made it the single most down regulated gene in this study. The S1-class bZIP transcription factor bZIP1 was down regulated more than twofold. Members of the $G$ class of bZIP transcription factors were up regulated. Out of the five bZIPs in this class, bZIP68, bZIP54 and bZIP55 were up regulated. Out of the five bZIPs in this class, bZIP68, bZIP54 and bZIP55 were up regulated 4, 6, and 20-fold, respectively. Analysis of the ATH1 probeset sequences of these genes revealed no significant similarities to the bZIP11 sequence, ruling out non-specific binding of the abundant bZIP11 transcript to these probesets.

\section{Overexpression of bZIP10 causes minor changes in gene expression}

To date little is known about the biological functions of C-class bZIPs. mRNA levels in protoplasts transfected with plasmid DNA encoding HA-tagged bZIP10, show that bZIP10 by itself has little capability to activate gene expression (Supplementary table S2 and Figure 4). Only seven genes (besides bZIP10 itself) were induced at least two-fold upon induction of bZIP10 levels. None of the induced genes showed a significant expression level increase above four-fold. Among the group of seven upregulated genes was SUC7, a proposed sucroseproton symporter.

Remarkably, bZIP25 expression was down regulated more than three-fold by bZIP10 transfection, as was the case after induction of bZIP11. The only other repressed gene was HSP18.2, mRNA levels of which dropped slightly below half of those in the control treatment.

\section{bZIPs have differential effects on gene expression}

The effects of induced levels of both bZIP10 and bZIP11 together were examined to explore possible synergistic effect of bZIP dimers. It was found that when bZIP10 was added in combination with bZIP11, the effect on gene expression levels was much larger than the combined effect of both individual transcription factors. In total, 645 genes were statistically significantly upregulated more than two-fold (Supplementary table S3 and Figure 5), whereas only 7 or 383 genes were upregulated by the single bZIP10 or bZIP11 transfections, respectively. Out of the 645 bZIP10+11 responsive genes 269 also responded to bZIP11 alone. Some of these 269 overlapping genes responded stronger when bZIP11 was complemented with bZIP10, others responded in the same way or weaker. The $2 S$ SEED STORAGE PROTEIN 1 gene (At4g27140) 

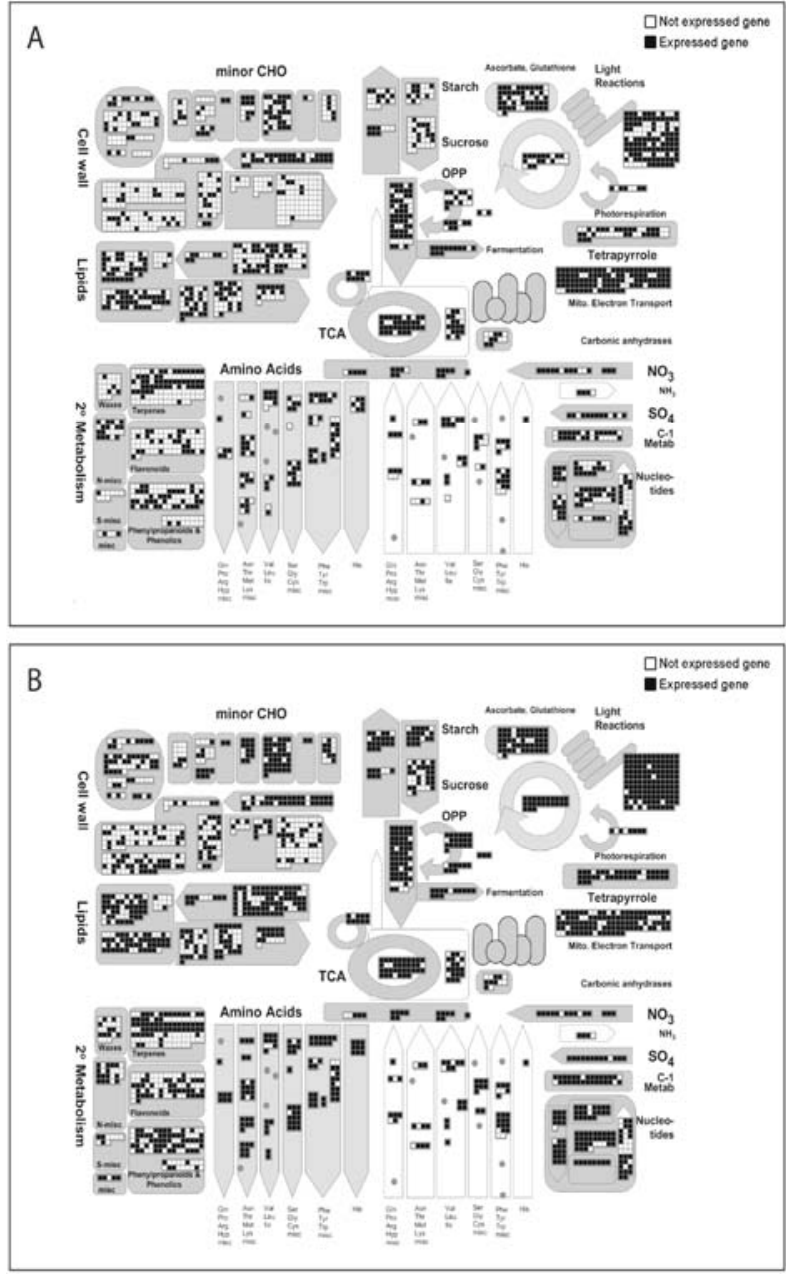

Figure 3. Gene expression in protoplasts is comparable to gene expression in seedlings The figure shows MAPMAN representations of all expressed genes in protoplasts (A) compared to 7-day-old seedlings (B). Grey squares represent genes expressed at levels that were statically significant above background levels in all slides of the respective experiments.

show the strongest combinatorial expression effect of bZIP10+11. It's expression was unaffected by bZIP11, but increased over 500fold when bZIP11 was complemented with bZIP10. In an essentially identical experimental setup both bZIP plasmids were transfected into protoplasts at half DNA concentrations (results not shown). Effects on gene expression of thesetransfections were similar to the ones described here.

Different bZIP dimers likely recognize different promoters. Promoters of genes affected by bZIP10+11 dimers were compared to promoters of genes affected by bZIP11 alone, probably forming bZIP11 homodimers (Figure 6). The one $\mathrm{Kb}$ upstream regions of the top-100 genes most significantly affected by either type of dimer were subjected to motif analysis. It was found that the different dimers affect genes with promoters that show different motif enrichment. In agreement with the data obtained for experiments on seedlings, an ACGT motif was found to be highly enriched in promoters of genes affect by dimers containing bZIP11.

\section{bZIPs have differential effects on biological processes}

The notion that different bZIP dimers affect different sets of genes and thereby different biological processes, was substantiated by gene ontology (GO) analysis. bZIP10 and bZIP11 were found to have a much stronger inducing than repressing effect on gene expression levels. Therefore, the most significantly up regulated genes (fold change $\geq 2$ ) were subjected to $\mathrm{GO}$ analysis. The topGO software package (Alexa and Rahnenfuhrer, 2006) was used to identify GO categories whose member genes showed a statistically different response after induction of bZIP levels. bZIP11 effects were compared to those of the empty vector. The effect of the bZIP10+11 combination was compared to the effect bZIP11 alone (Table 3). In agreement with the observation that different bZIPs have differential effects on gene expression, they also affect biological processes differentially. Some GO terms are induced after both transfections but the majority of the 20 most affected GO categories are unique for either bZIP10+11 or bZIP11 treatment.

\section{Discussion}

The transcription factor bZIP11 had significant effects on gene expression when overexpressed in Arabidopsis thaliana mesophyll protoplasts. Elevated levels of bZIP11 caused a total of 383 genes to show increased mRNA levels. Comparatively, few genes (36) were down regulated. This finding is in agreement with results obtained earlier. Also in seedlings, elevated levels of nuclear bZIP11 induced the expression of significantly more genes than it repressed (Hanson et al., 2008). In contrast to the situation in stably transformed seedlings, not all cells in the protoplast system overexpressed the bZIP proteins, due to the limit of transfection 
Table 2. Genes regulated by bZIP11 in protoplasts as well as seedlings

A list of genes with increased mRNA levels in protoplasts expressing a constitutive HA-tagged bZIP11, which were also induced in seedlings after bZIP11 induction (Hanson et al., 2008). Changes in mRNA levels are shown logarithmically.

\begin{tabular}{|c|c|c|c|c|c|}
\hline \multirow[b]{2}{*}{ AGI } & \multirow[b]{2}{*}{ Description } & \multicolumn{2}{|c|}{ Seedlings } & \multicolumn{2}{|c|}{ Protoplasts } \\
\hline & & Log2FC & adj.P.Val & Log2FC & adj.P.Val \\
\hline AT1G02660 & lipase class 3 family protein & 3.89 & $2.36 \mathrm{E}-09$ & 2.02 & $1.28 \mathrm{E}-09$ \\
\hline AT1G10070 & ATBCAT-2; branched-chain-amino-acid transaminase/ catal... & 5.21 & $6.61 \mathrm{E}-06$ & 1.03 & $2.55 \mathrm{E}-03$ \\
\hline AT1G15040 & glutamine amidotransferase-related & 11.41 & 1.13E-09 & 4.33 & $3.98 \mathrm{E}-14$ \\
\hline AT1G16850 & unknown protein & 2.79 & $2.04 \mathrm{E}-08$ & 4.62 & $2.37 \mathrm{E}-14$ \\
\hline AT1G18460 & lipase family protein & 6.06 & 3.47E-09 & 1.57 & $1.48 \mathrm{E}-06$ \\
\hline AT1G19450 & integral membrane protein, putative / sugar transporter... & 4.41 & $6.61 \mathrm{E}-07$ & 3.18 & $6.35 \mathrm{E}-06$ \\
\hline AT1G30820 & CTP synthase, putative / UTP--ammonia ligase, putative & 4.40 & $6.70 \mathrm{E}-06$ & 2.28 & $2.48 \mathrm{E}-06$ \\
\hline AT1G51090 & heavy-metal-associated domain-containing protein & 6.16 & $7.81 \mathrm{E}-07$ & 2.27 & 7.74E-07 \\
\hline AT1G56660 & unknown protein & 3.57 & $3.29 \mathrm{E}-08$ & 1.65 & $1.59 E-06$ \\
\hline AT1G60190 & armadillo/beta-catenin repeat family protein / U-box do... & 8.93 & $4.98 \mathrm{E}-07$ & 1.19 & 1.21E-04 \\
\hline AT1G62510 & protease inhibitor/seed storage/lipid transfer protein... & 2.20 & 5.17E-05 & 2.55 & 1.40E-10 \\
\hline AT1G64620 & Dof-type zinc finger domain-containing protein & 6.12 & $7.33 \mathrm{E}-10$ & 1.67 & $3.31 E-06$ \\
\hline AT1G71980 & protease-associated zinc finger (C3HC4-type RING finger... & 8.44 & $2.02 \mathrm{E}-07$ & 2.98 & $2.82 \mathrm{E}-08$ \\
\hline AT1G73120 & similar to hypothetical protein [Vitis vinifera] (GB:CA... & 18.37 & $9.21 \mathrm{E}-12$ & 3.66 & 2.39E-09 \\
\hline AT2G25200 & similar to unknown protein [Arabidopsis thaliana] (TAIR... & 2.44 & $3.21 \mathrm{E}-04$ & 1.88 & 7.60E-07 \\
\hline AT2G26600 & glycosyl hydrolase family 17 protein & 3.33 & $7.81 \mathrm{E}-07$ & 1.18 & $6.85 \mathrm{E}-06$ \\
\hline AT2G30600 & $\mathrm{BTB} / \mathrm{POZ}$ domain-containing protein & 2.36 & $1.51 \mathrm{E}-05$ & 2.03 & 5.13E-07 \\
\hline AT2G36310 & inosine-uridine preferring nucleoside hydrolase family... & 2.91 & $1.16 \mathrm{E}-07$ & 1.87 & 2.09E-06 \\
\hline AT2G39130 & amino acid transporter family protein & 2.88 & $5.16 \mathrm{E}-04$ & 2.72 & $1.03 \mathrm{E}-07$ \\
\hline AT2G39570 & ACT domain-containing protein & 5.00 & $3.69 \mathrm{E}-10$ & 1.45 & $3.41 E-08$ \\
\hline AT2G41830 & cyclin-related & 2.32 & $7.22 \mathrm{E}-03$ & 2.12 & 2.84E-08 \\
\hline AT2G47770 & benzodiazepine receptor-related & 44.60 & $9.21 \mathrm{E}-12$ & 2.81 & $1.40 \mathrm{E}-10$ \\
\hline AT3G29160 & AKIN11 (ARABIDOPSIS SNF1 KINASE HOMOLOG 11); protein ki... & 4.92 & $1.05 \mathrm{E}-05$ & 2.39 & 8.54E-11 \\
\hline AT3G30775 & ERD5 (EARLY RESPONSIVE TO DEHYDRATION 5); proline dehyd... & 13.39 & $3.47 \mathrm{E}-11$ & 3.18 & $5.30 \mathrm{E}-10$ \\
\hline AT3G43430 & zinc finger (C3HC4-type RING finger) family protein & 3.42 & $4.21 \mathrm{E}-06$ & 3.24 & 5.77E-10 \\
\hline AT3G47340 & ASN1 (DARK INDUCIBLE 6) & 2.11 & $4.80 \mathrm{E}-05$ & 1.34 & $2.66 \mathrm{E}-06$ \\
\hline AT3G53620 & ATPPA4 (ARABIDOPSIS THALIANA PYROPHOSPHORYLASE 4); inor... & 2.79 & $1.47 \mathrm{E}-03$ & 1.80 & $1.52 \mathrm{E}-07$ \\
\hline AT3G57520 & ATSIP2 (ARABIDOPSIS THALIANA SEED IMBIBITION 2); hydrol... & 2.28 & $1.39 \mathrm{E}-06$ & 1.56 & $4.66 \mathrm{E}-08$ \\
\hline AT4G23870 & similar to unknown protein [Arabidopsis thaliana] (TAIR... & 2.49 & $5.45 \mathrm{E}-04$ & 1.30 & $2.85 \mathrm{E}-06$ \\
\hline AT4G24040 & ATTRE1/TRE1 (TREHALASE 1); alpha, alpha-trehalase/ treha... & 12.49 & $3.23 E-11$ & 1.55 & 8.46E-10 \\
\hline AT4G28040 & nodulin MtN21 family protein & 5.37 & $3.87 \mathrm{E}-07$ & 2.66 & $2.02 \mathrm{E}-07$ \\
\hline AT4G33420 & peroxidase, putative & 2.35 & $1.73 E-05$ & 5.69 & $1.67 \mathrm{E}-13$ \\
\hline AT4G33700 & CBS domain-containing protein & 7.59 & 3.05E-10 & 4.26 & $3.30 \mathrm{E}-11$ \\
\hline AT4G33750 & unknown protein & 2.90 & $1.31 \mathrm{E}-07$ & 1.50 & 1.68E-07 \\
\hline AT4G35770 & SEN1 (DARK INDUCIBLE 1) & 25.14 & $9.02 \mathrm{E}-17$ & 1.52 & 4.51E-08 \\
\hline AT4G39660 & AGT2 (ALANINE:GLYOXYLATE AMINOTRANSFERASE 2); alanine-g... & 4.47 & $5.84 \mathrm{E}-08$ & 1.99 & 1.69E-05 \\
\hline AT5G04310 & pectate lyase family protein & 5.19 & $1.99 \mathrm{E}-10$ & 3.71 & 4.19E-11 \\
\hline AT5G08350 & GRAM domain-containing protein / ABA-responsive protein... & 5.98 & $1.29 \mathrm{E}-08$ & 5.03 & $1.20 \mathrm{E}-12$ \\
\hline AT5G15410 & DND1 (DEFENSE NO DEATH 1); calcium channel/ calmodulin... & 5.75 & $4.00 \mathrm{E}-11$ & 4.21 & $1.31 \mathrm{E}-12$ \\
\hline AT5G22460 & esterase/lipase/thioesterase family protein & 3.74 & $9.26 \mathrm{E}-09$ & 2.19 & 2.85E-09 \\
\hline AT5G22920 & zinc finger ( $\mathrm{C} 3 \mathrm{HC} 4$-type RING finger) family protein & 5.76 & $3.19 \mathrm{E}-08$ & 1.79 & 1.13E-05 \\
\hline AT5G58650 & PSY1 (PLANT PEPTIDE CONTAINING SULFATED TYROSINE 1) & 7.34 & $3.32 \mathrm{E}-10$ & 1.90 & $6.22 \mathrm{E}-04$ \\
\hline AT5G66170 & similar to unknown protein [Arabidopsis thaliana] (TAIR... & 7.08 & $9.21 \mathrm{E}-12$ & 4.91 & 1.13E-14 \\
\hline AT5G66650 & similar to unknown protein [Arabidopsis thaliana] (TAIR... & 4.81 & 3.44E-09 & 1.27 & 1.04E-03 \\
\hline
\end{tabular}

efficiency. This implies that the effects on genes that are repressed are limited to the cells that have been transfected. The drop in mRNA in those cells is potentially overshadowed by the mRNA still present in the untransfected cells. However, for bZIP25 it

was shown that its expression was lowered 3fold. Assuming total repression of bZIP25 in transfected protoplasts, this indicates a transformation efficiency of $66 \%$. Among the set of confirmed bZIP11 target genes were KIN11, and two dark inducible (DIN) genes, DIN1 and DIN6/ASN1. Of the 163 genes upregulated in the seedling- based micro-array experiment, 44 genes were upregulated in protoplasts. Even though statistically speaking this number is highly significant, from a biological standpoint one might ask why not more genes were confirmed in the protoplast system. The main reason may be found in the different tissue specificities inherent to the systems used. When studying gene expression in mesophyll protoplasts, mRNAs of genes specifically expressed in other tissues (e.g. roots or flowers) will not be present. Target genes identified in 7 day old seedlings that were not confirmed in protoplasts were expressed in other tissues than mesophyll cells. 

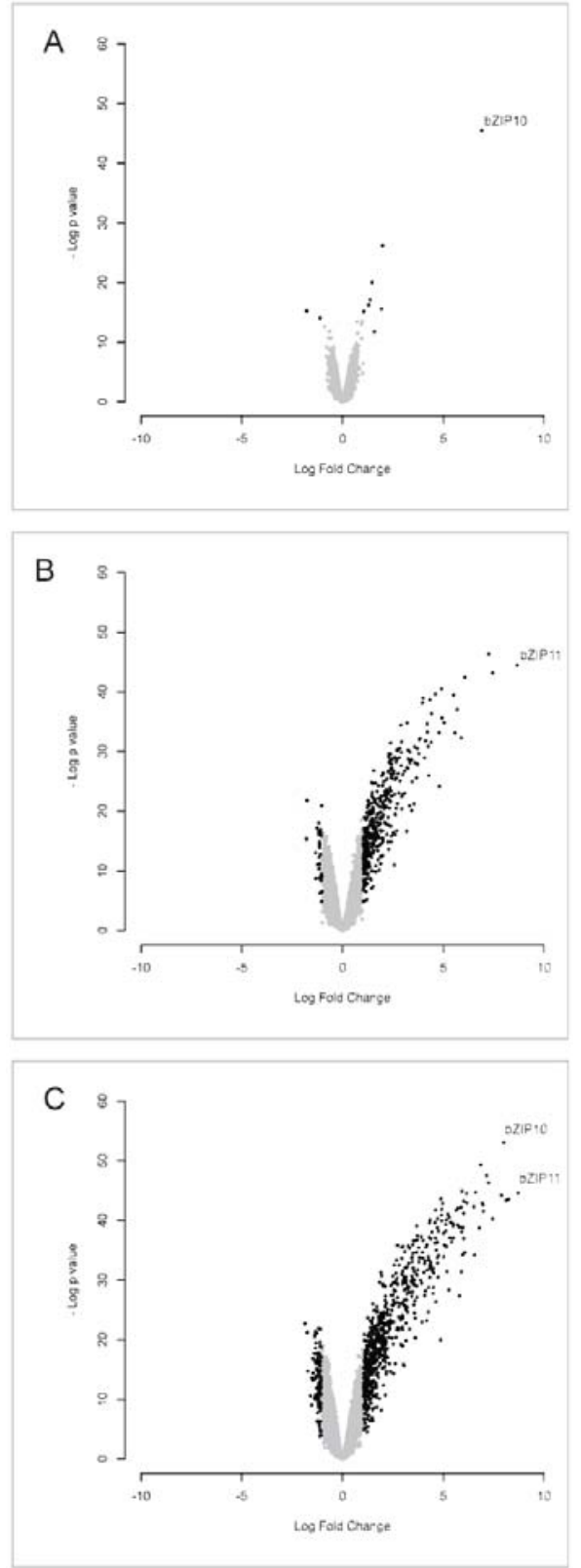

Figure 4. Differential gene expression in transfected protoplasts

The volcanoplots show the normalized relative signal changes in the protoplasts transfected with bZIP10 (A), bZIP11 (B) or bZIP10 and bZIP11 (C), in relation to the corresponding change in the protoplasts transfected with the empty vector. Logged signal changes are plotted against the $p$-value of that change. Dots with an average signal change of at least twofold are depicted in black.

Data from the AtGenExpress project (Schmid et al., 2005) revealed that in seedlings, $85 \%$ of the missing genes were expressed in roots (51), cotyledons (18), hypocotyls (3), or the shoot apex (1). Interestingly, among the newly identified targets of bZIP11 were other bZIPs. The S1-class bZIP transcription factor bZIP1 as well as the C-class bZIP transcription factor bZIP25 were down regulated more than twofold.
Three out of five members of the $G$ class of bZIP transcription factors were up regulated (bZIP68, bZIP54 and bZIP55). These results suggest a network in which bZIPs affect each other's expression.

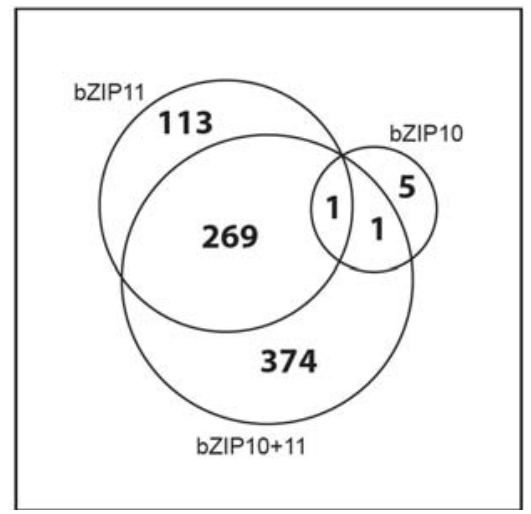

\section{Figure 5. Differential effects of bZIP dimers}

The Venn diagram shows the number of induced genes in protoplasts following transfection with 35S:bZIP constructs. bZIP11 expression led to activation of 113 genes that were not induced after co-expression of bZIP10 and bZIP11, indicating a response to dimers containing bZIP11 but not bZIP10. bZIP10 expression led to activation of 5 genes that were not induced after co-expression of bZIP10 and bZIP11, indicating a response to dimers containing bZIP10 but not bZIP11. Coexpression of bZIP10+11 led to activation of 374 genes that were not induced after induction of either bZIP10 or bZIP11 alone, indicating bZIP10+11 dimer specificity for the induction of these genes.

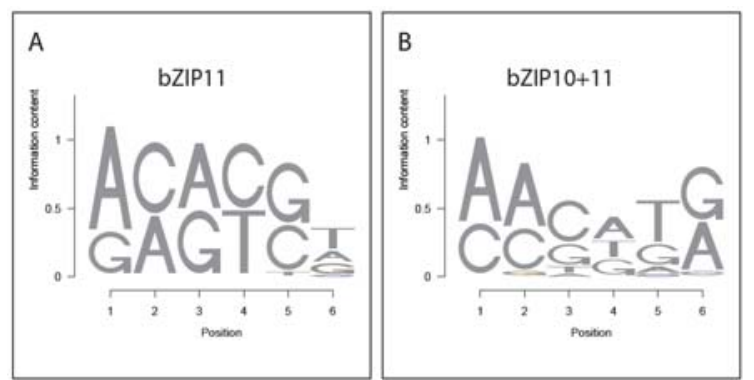

Figure 6. Motif analysis of promoters of bZIP targets.

The position weight matrixes of the 5 most significantly enriched motifs in the promoters of the 100 genes most significantly affected by dimers of bZIP11 (A) or bZIP10+11 dimers (B).

Compared to bZIP11, bZIP10 showed minimal effects on gene expression in protoplasts. Only 7 genes were upregulated, and 2 were downregulated by bZIP10. One possible reason for the smaller effect bZIP10 may be found in the fact that it was overexpressed to lower levels then bZIP10: 120-fold compared to over 400-fold. However, we expect both bZIPs to be saturated in terms of gene expression and protein translation. Furthermore, bZIP10+11 exerted quite dramatic effects on gene 
Table 3B. Differentially enriched GO terms based on biological process among genes affected by bZIPs

\begin{tabular}{|c|c|c|c|c|c|c|}
\hline \multirow[b]{2}{*}{ Go.ID } & \multirow[b]{2}{*}{ Term } & \multirow[b]{2}{*}{ Total in genome } & \multicolumn{2}{|c|}{ bZIP11 } & \multicolumn{2}{|c|}{ bZIP10+11 compared to bZIP11 } \\
\hline & & & Changed & p value & Changed & p value \\
\hline GO:0000038 & very-long-chain fatty acid metabolic pro... & 4 & - & - & 2 & 0.013 \\
\hline GO:0000304 & response to singlet oxygen & 5 & - & - & 2 & 0.021 \\
\hline GO:0000914 & phragmoplast formation & 1 & 1 & 0.022 & 1 & 0.049 \\
\hline GO:0002240 & response to molecule of oomycetes origin & 2 & 1 & 0.044 & - & - \\
\hline GO:0005513 & detection of calcium ion & 6 & - & - & 2 & 0.031 \\
\hline GO:0005991 & trehalose metabolic process & 8 & - & - & 3 & 0.005 \\
\hline GO:0006000 & fructose metabolic process & 2 & 1 & 0.044 & _- & - \\
\hline GO:0006560 & proline metabolic process & 7 & - & - & 3 & 0.003 \\
\hline GO:0006809 & nitric oxide biosynthetic process & 2 & 1 & 0.044 & - & - \\
\hline GO:0006833 & water transport & 4 & - & - & 2 & 0.013 \\
\hline GO:0006869 & lipid transport & 19 & 3 & 0.008 & 5 & 0.002 \\
\hline GO:0006875 & cellular metal ion homeostasis & 16 & 2 & 0.049 & - & - \\
\hline GO:0007020 & microtubule nucleation & 1 & 1 & 0.022 & - & - \\
\hline GO:0007155 & cell adhesion & 11 & - & - & 4 & 0.001 \\
\hline GO:0009269 & response to desiccation & 16 & - & - & 3 & 0.040 \\
\hline GO:0009744 & response to sucrose stimulus & 26 & 3 & 0.020 & 6 & 0.001 \\
\hline GO:0009826 & unidimensional cell growth & 34 & 5 & 0.006 & 6 & 0.020 \\
\hline GO:0009828 & cellulose and pectin-containing cell wal... & 4 & 2 & 0.003 & 3 & 0.000 \\
\hline GO:0009972 & cytidine deamination & 2 & 1 & 0.044 & - & - \\
\hline GO:0010103 & stomatal complex morphogenesis & 1 & 1 & 0.022 & - & - \\
\hline GO:0010165 & response to X-ray & 1 & 1 & 0.022 & - & - \\
\hline GO:0010231 & maintenance of seed dormancy & 1 & 1 & 0.022 & - & - \\
\hline GO:0010256 & endomembrane organization & 1 & 1 & 0.022 & - & - \\
\hline GO:0016126 & sterol biosynthetic process & 15 & - & - & 3 & 0.033 \\
\hline GO:0016567 & protein ubiquitination & 48 & - & - & 6 & 0.028 \\
\hline GO:0030397 & membrane disassembly & 1 & 1 & 0.022 & - & - \\
\hline GO:0030497 & fatty acid elongation & 2 & 1 & 0.044 & - & - \\
\hline GO:0042335 & cuticle development & 5 & - & - & 2 & 0.021 \\
\hline GO:0042545 & cell wall modification & 11 & - & - & 5 & 0.041 \\
\hline GO:0045449 & regulation of transcription & 445 & - & - & 30 & 0.041 \\
\hline GO:0046351 & disaccharide biosynthetic process & 15 & - & - & 3 & 0.033 \\
\hline GO:0046470 & phosphatidylcholine metabolic process & 1 & 1 & 0.022 & - & - \\
\hline GO:0046785 & microtubule polymerization & 1 & 1 & 0.022 & - & - \\
\hline GO:0048765 & root hair cell differentiation & 7 & - & - & 3 & 0.003 \\
\hline GO:0048767 & root hair elongation & 3 & 2 & 0.002 & - & - \\
\hline
\end{tabular}

gene expression, which also argues against this explanation. So far, bZIP10 is known to be involved in defense and cell death in Arabidopsis following infection with the pathogen Hyaloperonospora parasitica (Kaminaka et al., 2006). It is also known to be an ortholog of maize OPAQUE2, which participates in the regulation of seed storage protein genes (Lara et al., 2003). Accordingly, the expression of the 2S SEED STORAGE PROTEIN 1 increased over 500-fold in bZIP10+11 transfections. That effect dwarfs even the $35 \mathrm{~S}$ constitutive promoter driven expression increase of bZIP10. This confirms the involvement of bZIP10 in regulation of seeds storage protein, however it also implies that bZIP10 does not regulate the expression of this gene by itself, as the expression of the $2 S$ SEED STORAGE PROTEIN 1 remained unchanged in protoplasts only overexpressing bZIP10.

In order to bind DNA with their basic region, bZIP transcription factors need to form dimers with their ZIP domain. The eukaryotic bZIP transcription factors Jun and Fos are wellstudied examples. Sedimentation equilibrium studies and thermal unfolding experiments have revealed that dimer stability decreases in the order Jun-Fos $>$ Jun-Jun $>$ Fos-Fos (O'Shea et al., 1989). This has implications for the function of the dimers. While neither the Jun nor Fos monomer will bind the Jun/Fos DNA binding site (Turner and Tjian, 1989), the Jun, but not the Fos, homodimer will bind the DNA binding site (Neuberg et al., 1989). In analogy to this system, it has been shown that in Arabidopsis, C-class bZIPs predominantly dimerise with S1class bZIPs, but are unable to form homodimers (Ehlert et al., 2006a). S1-class bZIPs in contrast, can form homodimers, even though they prefer dimerization with the C-class. Induction of either bZIP10 (C-class) or bZIP11 (S1-class) levels in protoplasts resulted in 7 or 383 genes being statistically significantly up regulated more than 2 fold, respectively. If however both 
bZIP10 and bZIP11 levels were increased, 645 genes were statistically significantly up regulated more than 2 fold. This results in the distinction of three groups of genes: a group of 5 genes specifically responding to bZIP10 dimerised with a non-bZIP11 protein, a group of 113 genes specifically responding to bZIP11 homodimers or bZIP11 dimerised with a nonbZIP10 protein, and a group of 374 genes specifically responding to bZIP10 dimerised with bZIP11. The synergistic effect caused by both transcription factors is not due to a mere increase in the total amount of transcription factor protein present. In an independent experiment in which only half the amount of bZIP plasmid DNA was used, highly similar results to the ones presented here were obtained, and protein levels in both cases were found to be similar by western analysis (Figure 1). In this situation protoplasts would have a comparable amount of bZIP protein to that after a single plasmid transfection.

The synergistic effects of bZIP10 and bZIP11 provide evidence for the fact that different bZIP dimers have different functions. Considering the number of S1- and C-class bZIPs and the fact that they can be differentially regulated, a complex network of bZIP dimers emerges. Additionally, the regulation of the SNF1-related protein kinase KIN11 (At3g29160) by the bZIP10+11 dimer is intriguing as S1-class bZIPs have been claimed to be involved in stress mediated SNF1-related protein kinase 1 (SnRK1) signaling (Baena-Gonzalez et al., 2007). SnRK1 kinases play an important role in the regulation of transcription, metabolism and development in response to energy limitation, e.g. due to carbon starvation (Hardie, 2007., Baena-Gonzalez et al., 2007). The increased KIN11 expression in response to bZIP11 thus presents a possible positive feedback mechanism. Recently, T6P has been shown to be a regulator in SnRK1 signaling (Zhang et al., 2009). Interestingly, 6 genes involved in trehalose metabolism are affected in a way that might result in reduced T6P levels. The expression levels of four trehalose-6-phosphate phosphatase genes (TPP2, TPP5, TPP6 and TPP9), as well as that of the single trehalase gene (TRE1) were increased by the bZIP10/11 dimer. Furthermore, expression levels of a trehalose phosphatase/synthase (TPS8), were reduced. Likely, bZIP11 thus affects T6P levels, but this has not been tested. This effect of bZIP11 would be in agreement with the finding that sucrose can lead to a large (30-fold) and fast increase in T6P levels (Lunn et al., 2006), as sucrose will inhibit the production of S1-class of bZIPs. bZIP11 effects on trehalose metabolism could, therefore, represent another possible feedback mechanism in the sugar/SnRK1/bZIP pathway. Moreover, the effect of bZIP11 on trehalose metabolism was shown to be biologically relevant (Chapter 3). Induction of bZIP11 abolishes growth arrest of seedlings caused by high concentrations of trehalose in the medium. Taken together, these results suggest an intricate signaling network involving SnRKs and bZIPs is regulating growth and development by directing gene transcription, and metabolism in accordance with the nutritional status. In this complex system sugars have an impact at multiple levels.

\section{Materials and Methods}

\section{Construction of plasmid DNA}

For transient expression of bZIP transcription factors in protoplasts, $35 \mathrm{~S}$ driven $3 \mathrm{x} 5$, $\mathrm{HA}$ tagged bZIP constructs were made (Ehlert et al., 2006a). The bZIP transcription factors were made available through the REGIA project, and were cloned into a modified version of the pHBT vector using Gateway ${ }^{\circledR}$ technology (Invitrogen, http://www.invitrogen.com). The modified pHBT, designated pHBTL $\Delta$ GFP was created by NcoI/NotI- digestion, Klenow fill in and religation (Thorsten Heinekamp, pers. com.).

\section{Transient expression of bZIP transcription factors in protoplasts}

The protoplast preparation was adapted from Sheen (Sheen, 2001). Arabidopsis mesophyl protoplasts were isolated from leaves (the second and/or third/fourth pair) of 5 weeks old 
Col-0 (CS60000) plants grown on soil under long day (16h light / 8h dark) conditions. Leaves were placed in enzyme solution for $8.5 \mathrm{~h}(1 \%$ cellulase R10, 0.3\% macerozyme R10 (Yakult Honsha, Tokyo, Japan), 0.4 M mannitol, $20 \mathrm{mM}$ $\mathrm{KCl}, 10 \mathrm{mM} \mathrm{CaCl}_{2}, 20 \mathrm{mM}$ MES, 0.1\% BSA (Sigma A-6793), pH 5.7)). Protoplasts were collected and kept on ice in W5 medium (154 $\mathrm{mM} \mathrm{NaCl}, 125 \mathrm{mM} \mathrm{CaCl} 2,5 \mathrm{mM} \mathrm{KCl}, 2 \mathrm{mM}$ MES, $\mathrm{pH}$ 5.7) for $13 \mathrm{~h}$ in the growth cabinet. Protoplasts were transferred to MMG solution (0.4 M mannitol, $15 \mathrm{mM} \mathrm{MgCl}_{2}, 4 \mathrm{mM}$ MES pH 5.7), and subjected to PEG transfection. To $2.12 \times 10^{6}$ protoplasts, a total of $250 \mu \mathrm{g}$ plasmid DNA was added followed by a $30 \mathrm{~min}$. incubation in 1 volume of PEG solution $(40 \%$ PEG 3500, $3 \mathrm{ml} \mathrm{H}_{2} \mathrm{O}, 0.2 \mathrm{M}$ mannitol, $0.1 \mathrm{M}$ $\mathrm{CaCl}_{2}$ ). After transfection the samples were diluted with 2 volumes of W5 solution and pelleted at $100 \mathrm{~g}$ for $2 \mathrm{~min}$. Protoplasts were then resuspended in $4 \mathrm{ml}$ WI medium $(0.5 \mathrm{M}$ mannitol, $20 \mathrm{mM} \mathrm{KCl}, 4 \mathrm{mM}$ MES, $\mathrm{pH}$ 5.7), transferred to $5 \mathrm{~cm}$ Petri dishes pre-coated with $5 \%$ calf serum, and incubated for $6 \mathrm{~h}$ in the growth cabinet. After the incubation protoplasts were collected and 100-200 mg aliquots were flash frozen in liquid nitrogen.

\section{Western analysis}

For Western analysis, $100 \mathrm{mg}$ of plant material was ground to a fine powder in liquid nitrogen, and denatured in $300 \mu 1$ of buffer containing $4 \mathrm{M}$ urea, $16.6 \%$ glycerol, $5 \%$-mercaptoethanol and $5 \%$ SDS. Aliquots $(10 \mu \mathrm{l})$ were then fractionated on a $10 \%$ polyacrylamide gel. Blotting onto PVDF membrane (Millipore, Braunschweig, Germany) and immunodetection were carried out as described by Harlow and Lane (Harlow and Lane, 1988).

\section{RNA extraction and cDNA synthesis}

RNA was purified using the RNeasy kit (Qiagen, http://www.qiagen.com) and the RNA purity and integrity were confirmed by using a RNA 6000 Nano Assay (Agilent, http://www.home.agilent.com) and gel electrophoresis. Genomic DNA was removed using pretreatment of the total RNA with DNase (Fermentas, http://www.fermentas. com) and
cDNA was synthesized using anchored oligo-T primers (Biolegio, http:// www.biolegio.com), and MLV reverse transcriptase (Promega, http://www.promega. com) according to the manufacturer's instructions.

\section{Micro-array analysis}

cRNA labeling, hybridization, washing and scanning of Affymetrix Arabidopsis ATH1 GeneChips ${ }^{\circledR}$ (Affymetrix, http://affymetrix.com) was performed by ServiceXS (http://www.servicexs.com), according to Affymetrix OneCycle Lab protocols. Data from the micro-array were analysed statistically using the $\mathrm{R}$ language environment for statistical computing (http://www.r-project.org) version 2.7.1 and Bioconductor release 2.1 (Gentleman et al., 2004). Data were normalized using the robust multi-arrayexpression measure (RMA) in the Affy package (Gautier L., 2004). Differentially expressed genes were identified using the LIMMA package (Smyth et al., 2005). The obtained P-values were corrected for multiple testing errors using the $\mathrm{BH}$ procedure (Benjamini, 1995), yielding q-values. Lists of qvalues were transferred to Microsoft Excel ${ }^{\mathrm{TM}}$ and sorted. The GST sequences were aligned to the Tair7 gene model database of transcripts. Genes were classified as being differentially expressed if expression significantly $(\mathrm{q} \leq 0.02)$ changed. Genes with lower than two-fold expression level changes, even if statistically significant, were not considered to be differentially expressed. To compare the list of differentially expressed genes with other gene lists Microsoft Excel ${ }^{\mathrm{TM}}$ was used.

For gene ontology data genes were organized based on both biological process and molecular function annotations and several publicly available tools were used, topGO (Alexa and Rahnenfuhrer, 2006), Tair (http://www.arabidopsis.org/tools/bulk/go/) and MAPMAN (Thimm et al., 2004).

\section{QPCR}

Real-time PCR was performed using the 7900HT Fast Real-Time PCR System and Cybergreen ${ }^{\circledR}$ chemistry (Applied Biosystems). 
Expression levels were calculated relative to the phosphatase 2A (PP2A) regulatory subunit (At1g13320) (Czechowski et al., 2005) levels using the Q-gene method that takes the relative efficiencies of the different primer pairs into account (Muller et al., 2002). Primers were designed according to the recommendations of the PCR master-mix manufacturer (Applied Biosystems) or designed to be gene-specific by the CATMA consortium (http://www.catma.org). Full lists of primer sequences can be obtained from the authors.

\section{References}

Alexa, A., and Rahnenfuhrer, J. (2006). topGO:

Enrichment analysis for Gene Ontology (R package version 1.8.1).

Allemeersch, J., Durinck, S., Vanderhaeghen, R.,

Alard, P., Maes, R., Seeuws, K., Bogaert, T., Coddens, K., Deschouwer, K., Van Hummelen, P., Vuylsteke, M., Moreau, Y., Kwekkeboom, J., Wijfjes, A.H., May, S., Beynon, J., Hilson, P., and Kuiper, M.T. (2005). Benchmarking the CATMA microarray. A novel tool for Arabidopsis transcriptome analysis. Plant Physiol 137, 588-601.

Baena-Gonzalez, E., Rolland, F., Thevelein, J.M., and

Sheen, J. (2007). A central integrator of transcription networks in plant stress and energy signalling. Nature 448, 938-942.

Benjamini, Y.H., Y. (1995). Controlling the False Discovery Rate: A Practical and Powerful Approach to Multiple Testing. Journal of the Royal Statistical Society Series B, 269-287.

Birnbaum, K., Jung, J.W., Wang, J.Y., Lambert, G.M., Hirst, J.A., Galbraith, D.W., and Benfey, P.N. (2005). Cell type-specific expression profiling in plants via cell sorting of protoplasts from fluorescent reporter lines. Nat Methods 2, 615-619.

Czechowski, T., Stitt, M., Altmann, T., Udvardi, M.K., and Scheible, W.R. (2005). Genome-wide identification and testing of superior reference genes for transcript normalization in Arabidopsis. Plant Physiol 139, 5-17.

Ehlert, A., Weltmeier, F., Wang, X., Mayer, C.S., Smeekens, S., Vicente-Carbajosa, J., and Droge-Laser, W. (2006). Two-hybrid proteinprotein interaction analysis in Arabidopsis protoplasts: establishment of a heterodimerization map of group $\mathrm{C}$ and group $\mathrm{S}$ bZIP transcription factors. Plant J 46, 890-900.

Gautier L., C.L., Bolstad B.M., Irizarry R.A. (2004). affy---analysis of Affymetrix GeneChip data at the probe level. Bioinformatics 20, 307--315.

Gentleman, R.C., Carey, V.J., Bates, D.M., Bolstad, B., Dettling, M., Dudoit, S., Ellis, B., Gautier,
L., Ge, Y., Gentry, J., Hornik, K., Hothorn, T., Huber, W., Iacus, S., Irizarry, R., Leisch, F., Li, C., Maechler, M., Rossini, A.J., Sawitzki, G., Smith, C., Smyth, G., Tierney, L., Yang, J.Y., and Zhang, J. (2004). Bioconductor: open software development for computational biology and bioinformatics. Genome Biol 5, R80.

Hanson, J., Hanssen, M., Wiese, A., Hendriks, M.M., and Smeekens, S. (2008). The sucrose regulated transcription factor bZIP11 affects amino acid metabolism by regulating the expression of ASPARAGINE SYNTHETASE1 and PROLINE DEHYDROGENASE2. Plant J 53, 935-949.

Hardie, D.G. (2007). AMPK and SNF1: Snuffing Out Stress. Cell Metab 6, 339-340.

Harlow, E., and Lane, D. (1988). Antibodies: a laboratory manual. (Cold Spring Harbour, NY: Cold Spring Harbour Laboratory).

Jakoby, M., Weisshaar, B., Droge-Laser, W., VicenteCarbajosa, J., Tiedemann, J., Kroj, T., and Parcy, F. (2002). bZIP transcription factors in Arabidopsis. Trends Plant Sci 7, 106-111.

Kaminaka, H., Nake, C., Epple, P., Dittgen, J., Schutze, K., Chaban, C., Holt, B.F., 3rd, Merkle, T., Schafer, E., Harter, K., and Dangl, J.L. (2006). bZIP10-LSD1 antagonism modulates basal defense and cell death in Arabidopsis following infection. Embo J 25, 4400-4411.

Lara, P., Onate-Sanchez, L., Abraham, Z., Ferrandiz, C., Diaz, I., Carbonero, P., and VicenteCarbajosa, J. (2003). Synergistic activation of seed storage protein gene expression in Arabidopsis by $\mathrm{ABI} 3$ and two bZIPs related to OPAQUE2. J Biol Chem 278, 21003-21011.

Lunn, J.E., Feil, R., Hendriks, J.H., Gibon, Y., Morcuende, R., Osuna, D., Scheible, W.R., Carillo, P., Hajirezaei, M.R., and Stitt, M. (2006). Sugar-induced increases in trehalose 6phosphate are correlated with redox activation of ADPglucose pyrophosphorylase and higher rates of starch synthesis in Arabidopsis thaliana. Biochem J 397, 139-148.

Muller, P.Y., Janovjak, H., Miserez, A.R., and

Dobbie, Z. (2002). Processing of gene expression data generated by quantitative real-time RT-PCR. Biotechniques 32, 1372-1374, 1376, 1378- 1379.

Neuberg, M., Adamkiewicz, J., Hunter, J.B., and

Muller, R. (1989). A Fos protein containing the Jun leucine zipper forms a homodimer which binds to the AP1 binding site. Nature 341, 243245.

O'Shea, E.K., Rutkowski, R., Stafford, W.F., 3rd, and

Kim, P.S. (1989). Preferential heterodimer formation by isolated leucine zippers from fos and jun. Science 245, 646-648.

Rook, F., Gerrits, N., Kortstee, A., van Kampen, M., Borrias, M., Weisbeek, P., and Smeekens, S. (1998). Sucrose-specific signalling represses translation of the Arabidopsis ATB2 bZIP transcription factor gene. Plant J 15, 253-263.

Satoh, R., Fujita, Y., Nakashima, K., Shinozaki, K., 
and Yamaguchi-Shinozaki, K. (2004). A novel subgroup of bZIP proteins functions as transcriptional activators in hypoosmolarityresponsive expression of the ProDH gene in Arabidopsis. Plant Cell Physiol 45, 309-317.

Schmid, M., Davison, T.S., Henz, S.R., Pape, U.J.,

Demar, M., Vingron, M., Scholkopf, B., Weigel, D., and Lohmann, J.U. (2005). A gene expression map of Arabidopsis thaliana development. Nat Genet 37, 501-506.

Sheen, J. (2001). Signal transduction in maize and Arabidopsis mesophyll protoplasts. Plant Physiol 127, 1466-1475.

Smyth, G.K., Michaud, J., and Scott, H.S. (2005). Use of within-array replicate spots for assessing differential expression in microarray experiments. Bioinformatics 21, 2067-2075.

Thimm, O., Blasing, O., Gibon, Y., Nagel, A., Meyer,

S., Kruger, P., Selbig, J., Muller, L.A., Rhee, S.Y., and Stitt, M. (2004). MAPMAN: a userdriven tool to display genomics data sets onto diagrams of metabolic pathways and other biological processes. Plant J 37, 914-939.

Turner, R., and Tjian, R. (1989). Leucine repeats and an adjacent DNA binding domain mediate the formation of functional cFos-cJun heterodimers. Science 243, 1689-1694.
Weltmeier, F., Ehlert, A., Mayer, C.S., Dietrich, K., Wang, X., Schutze, K., Alonso, R., Harter, K., Vicente-Carbajosa, J., and Droge-Laser, W. (2006). Combinatorial control of Arabidopsis proline dehydrogenase transcription by specific heterodimerisation of bZIP transcription factors. Embo J 25, 3133-3143.

Weltmeier, F., Rahmani, F., Ehlert, A., Dietrich, K.,

Schütze, K., Wang, X., Chaban, C., Hanson, J., Teige, M., Harter, K., Vicente-Carbajosa, J., Smeekens, S., and Dröe-Laser, W. (2009). Expression patterns within the Arabidopsis C/S1 bZIP transcription factor network: availability of heterodimerization partners controls gene expression during stress response and development. Plant Mol Biol 69, 107-119.

Wiese, A., Elzinga, N., Wobbes, B., and Smeekens, S.

(2004). A conserved upstream open reading frame mediates sucrose-induced repression of translation. Plant Cell 16, 1717-1729.

Zhang, Y., Primavesi, L.F., Jhurreea, D., Andralojc,

P.J., Mitchell, R.A., Powers, S.J., Schluepmann, H., Delatte, T., Wingler, A., and Paul, M.J. (2009). Inhibition of Snf1- related protein kinase (SnRK1) activity and regulation of metabolic pathways by trehalose 6- phosphate. Plant Physiol. 


\section{$9 \quad$ General discussion}

This thesis focuses on the functional impact of the Arabidopsis C/S1 bZIP-TFs, which heterodimerise in a specific manner to activate target gene expression. Furthermore, colocalisation and protein stability of the group $\mathrm{C}$ and $\mathrm{S}$ AtbZIP-TFs were analysed.

\subsection{Specific heterodimerisation of group $C$ and group S1 bZIP transcription factors is mediated by the leucine zipper}

Protein-protein interactions are a tightly regulated network mediating cellular responses to environmental and developmental conditions. The complete sequencing of an increasing number of eukaryotic genomes has provided a wealth of information about the complexity of TFs. Compared to mammals or yeast little is known on specific protein-protein interactions of bZIP TFs and their target genes in planta,. Protein-protein interactions were analysed either by in vitro techniques such as co- immunoprecipitation and cofractionation by chromatography or in vivo using Y2H analysis (FIELDS and SONG, 1989), Bimolecular Fluorescence Complementation assay (BiFC) (KERPPOLA, 2006) or Fluorescence Resonance Energy Transfer (FRET) (POLLOK and HEIM, 1999). Y2H can be used as a high-throughput approach but conditions in yeast might not be the same as in plant cells and therefore might influence the protein-protein interaction results. To analyse AtbZIP heterodimerisation in planta, we established a Gateway ${ }^{\circledR}$-based two-hybrid system in plant protoplasts (EHLERT et al., 2006). This P2H approach is an artificial, but very efficient system and offers a number of advantages. It is an in vivo and in planta assay system which is fast and easy to handle. By using Gateway ${ }^{\circledR}$-compatible vectors the $\mathrm{P} 2 \mathrm{H}$ can be used as a high-throughput system. The P2H allows a quantitative read-out of protein-protein interactions. However, these data might be misleading, if one of the protein partners has an unspecific influence on the transcriptional read-out. As described for yeast systems, $\mathrm{BD}$ and $\mathrm{AD}$ fusion might have a negative effect on the protein interaction. Furthermore, interaction between proteins, which are normally not co-localised might be identified as well (EHLERT et al., 2006; WELTMEIER et al., 2009). 
A comparison between the results from $\mathrm{Y} 2 \mathrm{H}$ and $\mathrm{P} 2 \mathrm{H}$ system shows that many but not all interactions are identical. This finding can be partially be explained by plant-specific factors or conditions, which have an influence on the interaction and gene expression. In both systems, interactions between the group $\mathrm{C}$ and subgroup S1 AtbZIP TFs are prefer. This interaction pattern was also found for related bZIPs in plant species, such as parsley (Petroselium crispum) (RÜGNER et al., 2001) and tobacco (Nicotiana tabacum) (STRAHTMANN et al., 2001) indicating functional relevance of this observed heterodimerisation pattern.

Selected AtbZIPs of other groups, such as AtbZIP39 (group A), AtbZIP51 (group I) or all members of the subgroup S3 have not been found to dimerise with AtbZIP TFs of the group C or S1 (EHLERT, unpublished). Furthermore, two bZIPs of the subgroup S2 (AtbZIP42 and AtbZIP58) form only homodimers and heterodimers which each other (EHLERT et al., 2006). Altogether, this data shows that interaction within the C/S1 network is very specific.

BZIP dimer formation is mediated by the leucine zipper (SIBERIL et al., 2001; VINSON et al., 2002). Importantly, only the bZIP TFs of the group C and S1 contain an extraordinary long leucine zipper with seven to nine heptad repeats (Figure 8.1), which might explain heterodimerisation specificity (DEPPMANN et al., 2004). DEPPMANN et al., (2004) predicted by bZIP dimerisation specificity based on structural properties like length of the zipper, placement of specific aa and presence of interhelical electrostatic interactions. However, these bioinformatics predictions could not all be confirmed in this study (EHLERT et al., 2006). This suggests that additional regulatory mechanisms have an influence on the dimerisation, which requires further analyses.

To analyse the importance of the zipper dimerisation domain, the $\alpha$-helical structure of the zipper has been disrupted by changing the first two leucines aa of the ZIP repeats into Pro. E.g. the amino acids $\mathrm{Leu}_{188}$ and $\mathrm{Leu}_{195}$ of AtbZIP63 (group C) have been changed into Pro. This aa substitution leads to a decrease of AtbZIP63 dimerisation (WALTER et al., 2004). Which has been confirmed in Y2H studies for related mutants in AtbZIP53 (group S1) and AtbZIP10 (group C). In AtbZIP53, the aa $\mathrm{Leu}_{51}$ and Ala 72 have been exchanged (AtbZIP53pp) to disrupt $\alpha$-helical ZIP structure and consequently AtbZIP heterodimerisation and also target gene activation (WELTMEIER et al., 2006 Figure 8B). 
(a) gabcdef gabcdef gabcdef gabcdef gabcdef gabcdef gabcdef gabcdef gabcdef

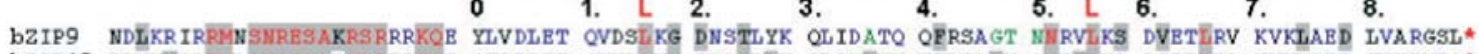
bZIP10 EDVKKSRRYISIRBSARRSRRRKOB QTSDLET QVWDIKG BHSSLIK QISNMNH KYDEAAV GIIRIIKA DIETIRA KVKMAEE TVKRVTG* bZIP25 TDVKRARPLI SIRESARRSRRRKOE CMNEPDT OVGOLRA EHSTLIN RUSDMNH KYDAAAV DIRTIRA DIETIRT KVKMAEE TVKRVTG*

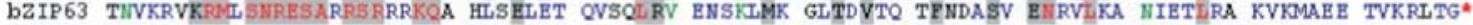

(b) gabcdef gabcdef gabcdef gabcdef gabcdef gabcdef gabcdef gabcdef gabcdef

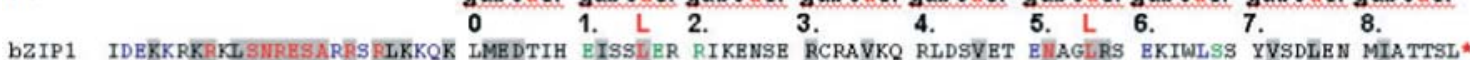

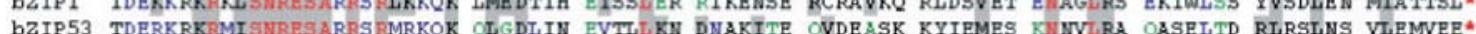

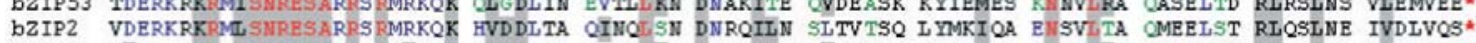

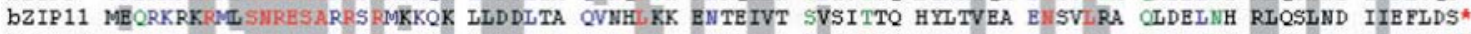

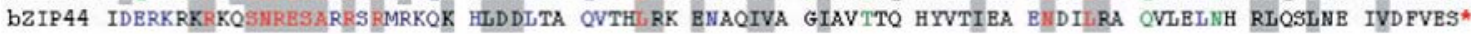

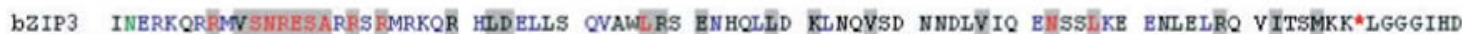

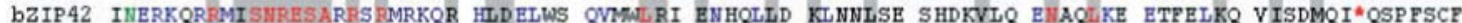

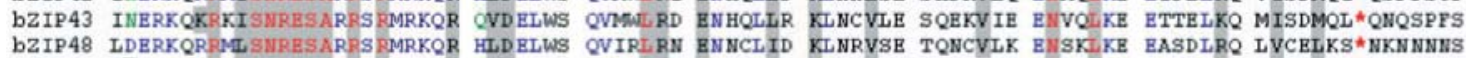
b2IP58 DE-RKQRAMISIRRESARPS SMRKQR HLDELWS OVIRLRT DNHCLMD KLNRVSE SHELALK E IAKIKB ETSDLRQ LISEIKS*HNEDDHS bZIP8 ER--KRR KV SIRRESARPS RMRKOR HMEELWS MEVQ IN KNKSLVD ELSQARE CYEKVIE EIMKL RE ENSKSRK^MIGEIGL NRFLSVE bZIP70 PEERRARPWV SIIRESARPS RMRKKK OIEELQQ QVEOLM LNHHLSE KVINLLE SNHOILQ ENSOLKE KVSSPHL LMADVLL*PMRNAES b2IP75 DEEKRLRPMR SMRESARPT RMRERM MKEGLOM OVKOLMA YNOFLYM KYISLLE YNHOILQ ENSOLKR TVSSPHD QYTISYG NHEGIIG

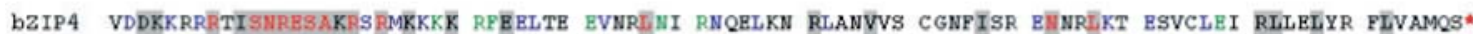

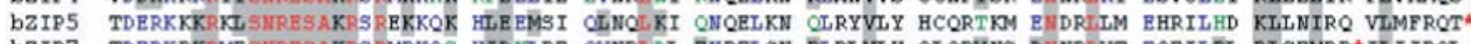
bZIP7 TDERKRKPME SIRRSAKPS RMRKQS HIDILRE OVNRLDL ENRELGN RLRLVLH QLQRVNS DINRIVT EQEILRL RLSEMRR* ILIIRQL b2IP6 TDDRKRKRMESMRESAKFSRMRKOR HID MLKD BANRLGL ENRELAM RLRIVLY NIALMCT DMMOILS EOEILRR RFLEMRQ*ILIFROL

Figure 8.1 EHLERT et al., 2006 (Figure 1a and b)

(a) Amino acid (aa) alignment of the bZIP domains of the group C members AtbZIP9 (At5g24800), AtbZIP10 (At4g02640), AtbZIP25 (At3g54620) and AtbZIP63 (At5g28770).

(b) Amino acid alignment of the bZIP domains of the group S members. Conserved aa are depicted in red The positions of the aa in the heptad repeats of the leucine zipper are labelled with gabcdef (LANDSCHULZ et al., 1988). Termination of the a-helical ZIP domain as predicted by DEPPMANN et al. (2004) is indicated.

This suggests that both dimerisation partners have to be compatible to form a transcriptional active heterodimer. The formation of bZIP homo- or heterodimers provides a tremendous combinatorial flexibility to a regulatory system (SIBERIL et al., 2001; VINSON et al., 2002). However, to gain specificity, some heterodimers could be preferred, as demonstrated for the interaction of $\mathrm{C}$ and S1 bZIPs. Analysis of all possible combinations of the 75 AtbZIP members is very labor-intensive. Therefore, we have created a high throughput protoplast transfection assay in microtiter plates (unpublished results). Combining the Gateway ${ }^{\circledR}$-compatible $\mathrm{P} 2 \mathrm{H}$ approach with this method will significantly speed-up the analysis.

However, further studies are important to define heterodimerisation partners in functional studies as well as improving bioinformatic tools for heterodimer prediction. 


\subsection{Expression patterns control abundance of bZIP monomer and therefore heterodimer formation}

In order to form heterodimers, the group $\mathrm{C}$ and $\mathrm{S} 1 \mathrm{bZIP}$ TFs have to be co-expressed and co-localised. Hence, co-expression was studied making use of bZIP promoter:GUS lines (WELTMEIER et al., 2009) and array expression data (ZIMMERMANN et al., 2004). For the members of the $\mathrm{C} / \mathrm{S} 1$ heterodimerisation network clearly overlapping expression patterns (e.g. in seedlings) as well as distinct differences (e.g. seed development) have been observed. As an example, AtbZIP53 has been shown to be expressed in seeds and to heterodimerise with AtbZIP10 and AtbZIP25 to regulate MAT genes expression (ALONSO et al., 2009). The amount of bZIP dimerisation partners in individual cells has a significant influence on heterodimerisation and target gene expression. Ectopic expressions of AtbZIP53, AtbZIP63 or AtbZIP9 under the control of the 35S promoter (Pro35S) mediates activation of 2S2 albumin transcription in seedlings, where this gene is normally not expressed (WELTMEIER et al., 2009 Figure 3). These results demonstrate that AtbZIP63 and AtbZIP9 can also activate 2S2 albumin transcription but their tissue-specific expression pattern limits their function in planta. To summarise several bZIP heterodimers have the possibility to activate target gene transcription in planta, but this function is essentially confined by co-expression and co-localisation of different bZIP partners. Although this dataset (WELTMEIER et al. 2009) provides a framework to decide which heterodimers might be formed in a particular plant tissue, predictions are difficult, because additional regulatory mechanisms influence the TFs.

\subsection{Heterodimerisation between members of the C/S1 network controls target gene expression in stress response, primary metabolism and seed development}

As demonstrated by WELTMEIER et al., (2006) and ALONSO et al., (2009), strong gene activation was obtained for AtbZIP53/10 and AtbZIP53/25 heterodimers whereas none of these bZIPs exhibited strong activation capacity on its own. Specific heterodimerisation may act as a key mechanism to modulate transactivation properties in planta which is referred as the HIT-mechanism (heterodimer-induced transactivation). A complete analysis of all group $\mathrm{C} / \mathrm{S} 1$ interactions shows that the HIT mechanism is a typical feature of distinct 
heterodimers, particularly observed between AtbZIP25, AtbZIP10 and most S1 group members, but also inside the group C (AtbZIP10/63). These data suggest that HIT is general important for the function of the C/S1 network (WELTMEIER et al., 2006).

The functional impact of C/S1 bZIP heterodimers has been demonstrated for several examples. Heterodimers containing AtbZIP1 or AtbZIP53 function as crucial regulators in starvation response by regulating genes of amino acid (aa) metabolism (DIETRICH et al., unpublished). The expression of genes involved in the biosynthesis of asparagine (Asn) and the catabolism of branched chain aa is controlled by C/S1 bZIPs. Furthermore, we demonstrated the functional impact of bZIP heterodimers for the ProDH gene encoding Proline Dehydrogenase during rehydration after recovery from osmotic stress (WELTMEIER et al., 2006).

AtbZIP53 is the S1 member with the highest expression in mid and late maturation phases. We were able to demonstrate that AtbZIP53 plays a pivotal role in quantitative control of MAT gene expression, such as SSP and LEA genes. Interestingly, by heterodimerisation of AtbZIP53 with the group C TFs AtbZIP10 or AtbZIP25, the binding activity to target promoters is increased (ALONSO et al., 2009).

\subsection{C/S1 heterodimer formation controls DNA binding and interactions with other transcription factors}

Dimers of TFs affect target gene expression in different ways. The abundance of bZIP monomers is regulated by environmental and developmental conditions, which influence the heterodimerisation possibilities. By heterodimerisation the DNA-binding specificity, affinity and transactivation properties of the bZIPs might be altered (NAAR et al, 2001).

E.g. AtbZIP53 and AtbZIP10 are co-expressed under hypoosmotic conditions, interact with high affinity and lead to a strong activation of the ProDH by binding to ACTCATelement (WELTMEIER et al., 2006). This is in agreement with microarray results, demonstrating that among 121 rehydration-inducible genes, $48 \%$ harbour the ACTCAT motif in their promoters (OONO et al, 2003). This indicates that this motif is of general importance with respect to gene regulation during recovery after osmotic stress.

Detailed titration experiments in protoplasts and ELISA assays demonstrate the effect of heterodimerisation between AtbZIP53/10 and show that the binding activity of AtbZIP53 
is higher in the presence (than in the absence) of AtbZIP10 (ALONSO et al., 2009). Accordingly, in the titration experiment the 1:1 ratio of AtbZIP53 and AtbZIP10 leads to the highest target gene activity. These data clearly support that specific heterodimers binds specific cis-elements to activate target gene transcription. In addition, we observed, that co-expression of AtbZIP53 and AtbZIP10 leads to enhanced protein levels of the corresponding bZIPs, suggesting that heterodimer formation might stabilise the proteins from degradation. This provides an additional mechanism that contributes to the observed synergistic effect on transcription (ALONSO et al., 2009).

The AtbZIP11 protein is able to form heterodimers with other bZIP proteins in Arabidopsis protoplasts and yeast cells (EHLERT et al., 2006). In planta, over-expression of AtbZIP11 leads to strong changes in gene expression, which is probably caused by homodimerisation. But effects of heterodimers involving endogenously present bZIPs cannot be excluded (EHLERT et al., 2006). To analyse whether different bZIP dimers recognise different promoter-cis-elements, we transformed protoplasts with AtbZIP11 and its dimerisation partner AtbZIP10 to perform microarray analysis.

A significantly altered set of target genes was induced by co-expression of AtbZIP11 and AtbZIP10. The sequences of promoters affected by the AtbZIP11/10 heterodimer were compared to promoters of genes affected by AtbZIP11 or AtbZIP10 alone (HANSSEN et al., unpublished). Interestingly, expression of AtbZIP11 itself leads to enrichment of a different set of promoter cis-elements (Figure 8.2) when compared to the AtbZIP11/10 heterodimer (HANSSEN et al., unpublished). This data suggests that particular bZIP dimers bind specific cis-elements and therefore activate different sets of target genes. It is important to verify these results by qPCR of stable transformed seedlings (HANSON et al., 2008).

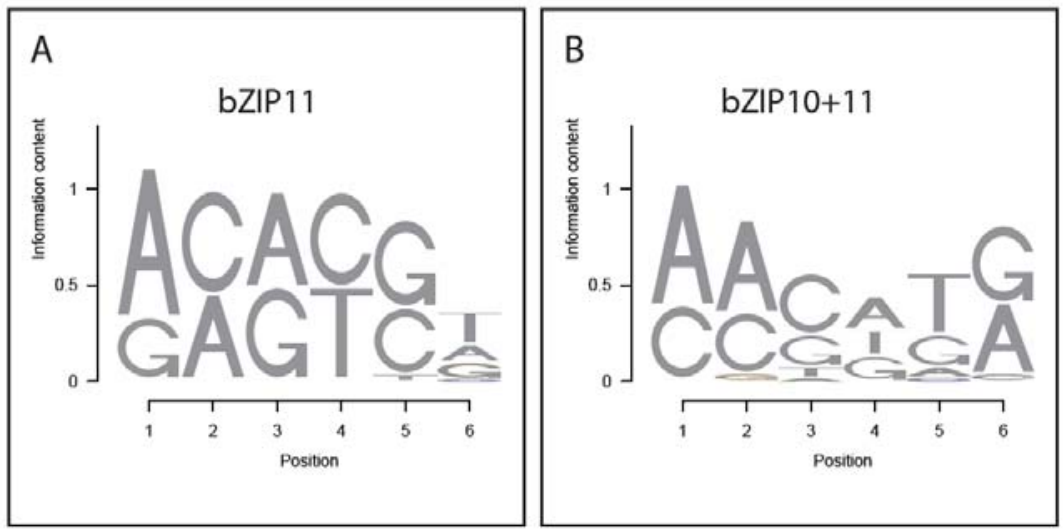

Figure 8.2 (HANSSEN et al., unpublished (Figure 4.6))

The position weight of the 5 most significantly enriched motifs in the promoters of the 100 most significantly affected genes by dimers of AtbZIP11 (A) or AtbZIP11/ 10 heterodimers (B). 
In vivo and in vitro analysis demonstrate that AtbZIP53/10 binds the G-box element of the SSP promoter such as 2S2 albumin, where the G-box element was mutated to prevent AtbZIP DNA binding (ALONSo et al., 2009). In additional to the G-box, another regulatory cis-element was found by sequence analysis, the so called RY-box (ALONSO et al., 2009). ABI3 is an important regulator of gene expression in seeds of Arabidopsis (GIRAUDAT et al., 1992; PARCY et al., 1994) and it has been shown that ABI3 binds the RY element (MÖNKE et al., 2004). A functional interplay between G-box and RY-element in MAT gene regulation has been previously described (EZCURRA et al., 2000; LARA et al., 2003). Furthermore, ABI3 is able to interact with AtbZIP10 or AtbZIP25 (LARA et al., 2003), but no interaction could be detected between AtbZIP53. Protoplast-three-hybrid $(\mathrm{P} 3 \mathrm{H})$ and $\mathrm{BiFC}$ analyses confirmed that AtbZIP53/10 or AtbZIP53/25 heterodimers interact with $\mathrm{ABI} 3$. A ternary complex formation would explain transcription of the 2S2 albumin gene which is enhanced by combination of bZIP heterodimers and ABI3 (ALONSO et al., 2009).

To summarise, bZIP heterodimers activate target genes by interaction with other TFs.

\subsection{Members of the C/S1 heterodimers differ in their properties but show partial redundancy}

Recent analysis of single bZIP ko plants show only a small effect on target gene expression (ALONSO et al., 2009; DIETRICH et al., unpublished). We speculate that partially redundant bZIP proteins can substitute for the missing TFs. The heterodimers AtbZIP53/10 and AtbZIP53/25 regulate SSP genes during seed maturation. AtbZIP53 is closely homologous to AtbZIP1. But the 2S2 albumin activation by the AtbZIP1/10 heterodimer was less pronounced in comparison to AtbZIP53/10, but leads to a stronger activation than AtbZIP1/25 heterodimers. Accordingly, histochemical analysis of ProAtbZIP1:GUS plants revealed by staining the expression of AtbZIP1 in the silique valves but not the in seeds as described for AtbZIP53 (see WELTMEIER et al., 2009 Figure 1b). Therefore, expression data as well as activation properties suggest that AtbZIP1 and AtbZIP53 are not redundant in respect of MAT gene regulation (WELTMEIER et al., 2009). In contrast, overlapping functions exist for AtbZIP1 and AtbZIP53 in regulation of ProDH and ASN1 in response to low energy stress (DIETRICH et al., unpublished). These findings demonstrate that the TFs of the $\mathrm{C} / \mathrm{S} 1$ network partly overlap with respect to their TF properties but clearly differ in 
function, due to specific expression and heterodimerisation patterns (WELTMEIER et al., 2009). Multiple bZIP T-DNA insertion lines lead to a significant impairment in target gene transcription during starvation response (DIETRICH et al., unpublished). However, further research is needed to elucidate how the loss of single bZIP functions can be substituted by other members of the network.

The work can be summarised in the following model:

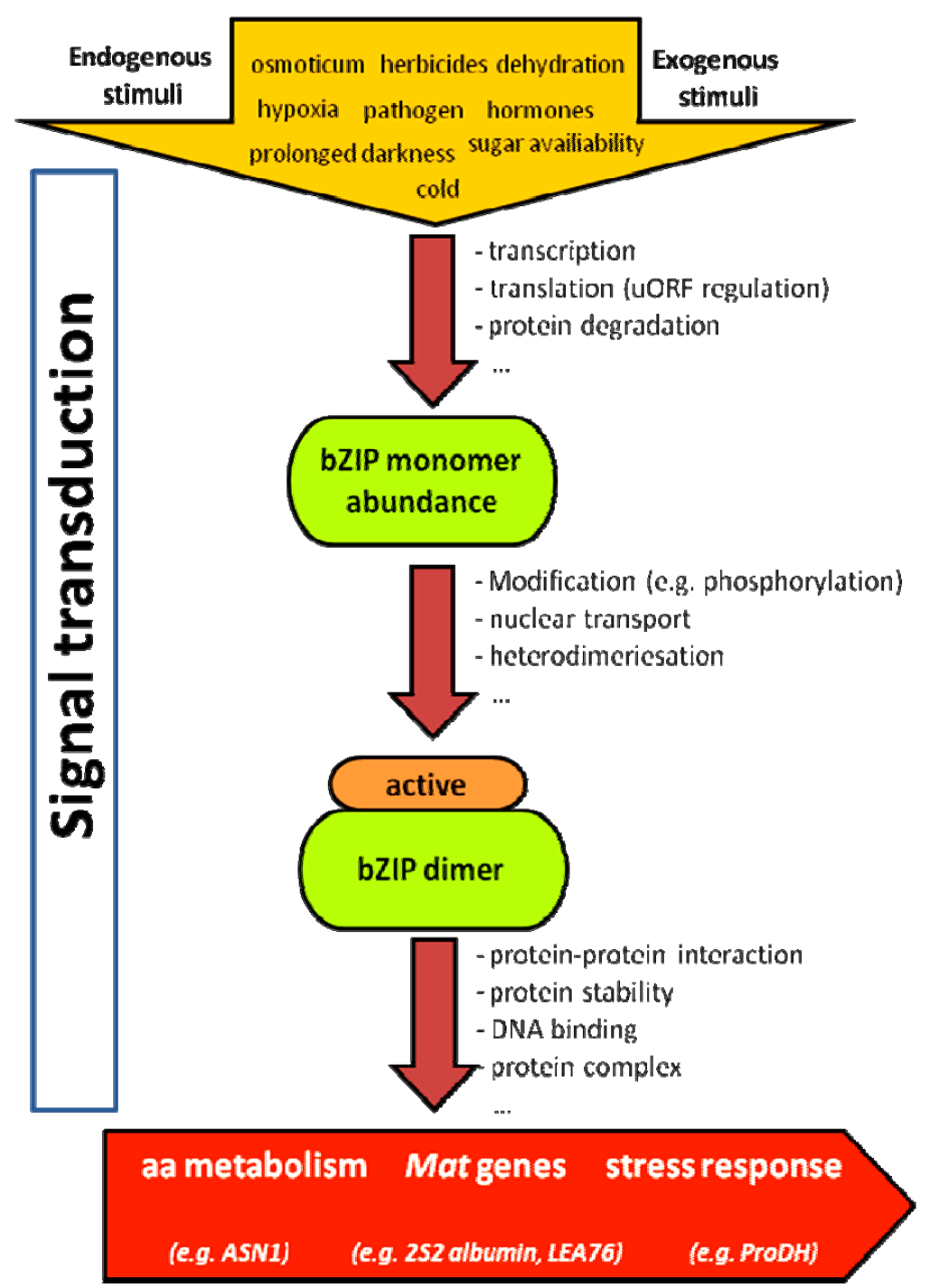

Figure 8.3 Model of the possible signal transduction pathway.

This overview shows that different endogenous or exogenous stresses result in a change of active AtbZIP monomer abundance. This leads to new dimmer- or complex formation and activate target gene expression. 


\section{References}

Abate, C, Marshak, D.R. and Curran, T. (1991): Fos is phosphorylated by p34cdc2, cAMPdependent protein kinase and protein kinase $\mathrm{C}$ at multiple sites clustered within regulatory regions. Oncogene 6, 2179-85.

Aguan, K., Sugawara, K., Suzuki, N. and Kusano, T. (1993): Low- temperaturedependent expression of a rice gene encoding a protein with leucin- zipper motif. Mol Gen Genet, 240, 1-8.

Alonso, R., Oñate-Sánchez, L., F., W., Ehlert, A., Diaz, I., Dietrich, K., VicenteCarbajosa, J., and Dröge-Laser, W. (2009): A pivotal role of the basic leucine zipper transcription factor AtbZIP53 in the regulation of seed maturation gene expression based on heterodimerisation and protein complex formation. Plant Cell

Armstrong, G.A., Weisshaar, B. and Hahlbrock, K. (1992): Homodimeric and heterodimeric leucine zipper proteins and nuclear factors from parsley recognize diverse promoter elements with ACGT cores. Plant Cell, 4, 525-537.

Baena-Gonzalez E, Rolland F, Thevelein JM, Sheen J (2007): A central integrator of transcription networks in plant stress and energy signalling. Nature 448: 938-942.

Bassham, D. C., Laporte, M., Marty, F., Moriyasu, Y., Ohsumi, Y., Olsen, L. J. and Yoshimoto, K. (2006): Autophagy in development and stress responses of plants. Autophagy.2 2-11.

Baena-Gonzalez, E., and Sheen, J. (2008): Convergent energy and stress signaling. Trends Plant Sci. 13: 474-482.

Baxevanis, A.D. and Vinson, C.R. (1993): Interactions of coiled coils in transcription factors: where is the specificity? Curr. Opin. Genet. Dev. 3, 278-285.

Chae, K. S., Gladys Y. P. K. and Stuart E. D. (2007): Tyrosine Phosphorylation of cGMPGated Ion Channels Is under Circadian Control in Chick Retina Photoreceptors. IOVS, 48, No. 2

Chasar, L.C. (2002): Implications of environmental change for energy flow through natural systems: Wetlands and coastal zones. Diss. Abst. Int. Pt. B - Sci. \& Eng. 3, 1237.

Chuang, C.- F. Running, M. P., Williams, R. W. and Meyerowitz E. M. (1999): The PERIANTHIA gene encodes a bZIP protein involved in the determination of floral organ number in Arabidopsis thaliana. Genes \& Dev. 13: 334-344

Ciceri, P., Gianazza, I. E., Lazzari, B., Lippoli, G., Genga, A., Hoschek, G., Schmidt, R. J. and Viottiag, A. (1997): Phosphorylation of opaque2 changes diurnally and its DNA binding activity impacts. Plant Cell, 9, 97-108. 
Cross, J. M., von Korff, M., Altmann, T., Bartzetko, L., Sulpice, R., Gibon, Y., Palacios,N. and Stitt, M. (2006): Variation of enzyme activities and metabolite levels in 24 Arabidopsis accessions growing in carbon-limited conditions. Plant Physiol, 142, 1574-1588

Delauney AJ, Hu CA, Kishor PB, Verma DP (1993): Cloning of ornithine deltaaminotransferase cDNA from Vigna aconitifolia by trans-complementation in Escherichia coli and regulation of proline biosynthesis. J Biol Chem 268: 18673-18678

De Pater, S., Katagiri, F., Kijne, J. and Chua, N. H. (1994): bZIP proteins bind to a palindromic sequence without an ACGT core located in a seed- specific element of the pea lectin promoter. Plant J. 6, 133- 140.

Deppmann, C.D., Acharya, A., Rishi, V., Wobbes, B., Smeekens, S., Taparowsky, E.J. and Vinson, C. (2004): Dimerization specificity of all 67 B-ZIP motifs in Arabidopsis thaliana: a comparison to Homo sapiens B-ZIP motifs. Nucleic Acids Res. 32, 3435-3445.

Djamei, A., Pitzschke, A., Nakagami, H., Rajh, I. and Hirt H. (2007): Trojan Horse Strategy in Agrobacterium Transformation: Abusing MAPK Defense Signaling. Science 318. No $5849,453-456$

Ehlert A, Weltmeier F, Wang X, Mayer CS, Smeekens S, Vicente- Carbajosa J, Dröge-Laser W (2006): Two-hybrid protein-protein interaction analysis in Arabidopsis protoplasts: establishment of a heterodimerisation map of group $\mathrm{C}$ and $\mathrm{S}$ bZIP transcription factors. Plant J 46:890-900.

Ezcurra, I., Wycliffe, P., Nehlin, L., Ellerstrom, M., and Rask, L. (2000):

Transactivation of the Brassica napus napin promoter by ABI3 requires interaction of the conserved $\mathrm{B} 2$ and $\mathrm{B} 3$ domains of $\mathrm{ABI} 3$ with different cis-elements: $\mathrm{B} 2$ mediates activation through an ABRE, whereas B3 interacts with an RY/G-box. Plant J. 24: 57-66.

Fields, S. and Song, O. (1989): A novel genetic system to detect protein-protein interactions. Nature, 340, 245-246.

Fong, J.H., Keating, A.E. and Singh, M. (2004): Predicting specificity in bZIP coiled-coil protein interactions. Genome Biol. 5, R11.

Foster, R., Izawa, T. and Chua, N. H. (1994): Plant bZIP proteins gather at ACGT elements. Faseb J, 8, 192- 200.

Fukazawa, J., Sakai, T., Ishida, S., Yamaguchi, I., Kamiya, Y. and Takahashi, Y. (2000): Repression of shoot growth, a bZIP transcriptional activator, regulates cell elongation by controlling the level of gibberellins. Plant Cell, 12, 901- 915.

Giraudat, J., Hauge, B.M., Valon, C., Smalle, J., Parcy, F., and Goodman, H.M. 
(1992): Isolation of the Arabidopsis ABI3 gene by positional cloning. Plant Cell

Guilioni, L., Wery, J. and Lecoeur J. (2003): High temperature and water deficient may reduce seed number in field pea purley by decrease plant growth rate. Plant Biology 30, 1151 - 1164

Gutierrez, L., Van Wuytswinkel, O., Castelain, M., and Bellini, C. (2007): Combined networks regulating seed maturation. Trends Plant Sci. 12: 294-300.

Halazonetis, T. D., Georgopoulos, K., Greenberg, M. E. and Leder P. (1988): c- Jun dimerises with itself and with c- Fos, forming complexes of different DANN binding affinities. Cell 55, 917- 924.

Halford, N. G. and Paul, M. J. (2003): Carbon metabolite sensing and signaling. Plant Biotechn. J. 1. 381- 398

Halford, N. G., Hey, S., Jhurreea, D., Laurie, S., McKibbin, R. S., Paul, M. and Zhang, Y. (2003): Metabolic signalling and carbon partitioning: role of Snf1-related (SnRK1) protein kinase. J. of Experimental Botany, 54, 467-475,

Halford, S. E., and Marko, J. F. (2004): How do site-specific DNA-binding proteins find their targets? Nucleic Acids Res. 32, 3040-52.

Hanson J, Hanssen M, Wiese A, Hendriks MWB, Smeekens S (2008): The sucrose regulated transcription factor bZIP11 affects amino acid metabolism by regulating the expression of Asparagin synthase1 and Proline dehydrogenase 2. Plant J 53:935-949.

Hardie, D.G. (2007): AMPK and SNF1: Snuffing Out Stress. Cell Metab 6, 339-340.

Heinekamp, T., Kuhlmann, M., Lenk, A., Strathmann, A. and Dröge- Laser,W. (2002): The tobacco bZIP transcription factor BZI-1 binds to G-box elements in the promoters of phenylpropanoid pathway genes in vitro, but it is not involved in their regulation in vivo. Mol. Genet. Genomics, 267, 16-26.

Hoekstra FA, Golovina EA, Buitink J (2001): Mechanisms of plant desiccation tolerance. Trends Plant Sci 6: 431-438

Hrabak, E. M., Chan C. W. M., Gribskov, M., Harper, J. F., Choi, J. H., Halford, N., Kudla, J., Luan, S., Nimmo, H. G., Sussman, M. R., Thomas, M., WalkerSimmons K., Zhu, J. K. and Harmon A. C. (2003): The Arabidopsis CDPK-SnRK Superfamily of Protein Kinases. Plant Physiology 132: 666-680

Hughes, D. W. and Galau, G. A. (1989): Temporally modular gene expression during cotyledon development. Genes Dev, 3, 358- 369.

Hurst, H. C. (1995): Transcription factors 1; bZIP proteins. Protein Profile, 2, 101- 168

Jakoby, M., Weisshaar, B., Dröge-Laser, W., Vicente- Carbajosa, J., Tiedemann, 
J., Kroj, T. and Parcy, F. (2002): bZIP transcription factors in Arabidopsis. Trends Plant Sci. 7, 106-111.

Kaminaka H, Nake C, Epple P, Dittgen J, Schütze K, Chaban C, Holt BF, Merkle T, Schäfer E, Harter K, Dangl J (2006): bZIP10- LSD1 antagonism modulates basal defense and cell death in Arabidopsis following infection. EMBO J 25:4400-4411.

Kerppola (2006): Design and Implementation of Bimolecular Fluorescence Complementation (BiFC) Assays for the Visualization of Protein Interactions in Living Cells. Nat. Protoc. 1, 1278- 1286

Kouzarides T, Ziff E (1988): The role of the leucine zipper in the fos-jun interaction. Nature 336: $646-651$

Kusano T, Berberich T, Harada M, Suzuki N, Sugawara K (1995): A maize DNAbinding factor with a bZIP motif is induced by low temperature. Mol Gen Genet 248:507-517.

Landschulz, W.H., Johnson, P.F. and McKnight, S.L. (1988): The leucine zipper: a hypothetical structure common to a new class of DNA binding proteins. Science, 240, 1759-1764.

Lam H. M., Peng S. S. and Coruzzi G. M. (1994): Metabolic regulation of the gene encoding glutamine- dependent asparagine synthetase in Arabidopsis thaliana. Plant Physiol 106:1347-1357.

Lara, P., Onate-Sa'nchez, L., Abraham, Z., Ferrandiz, C., Diaz, I., Carbonero, P., and Vicente-Carbajosa, J. (2003): Synergistic activation of seed storage protein gene expression in Arabidopsis by ABI3 and two bZIPs related to OPAQUE2. J. Biol. Chem. 278: $21003-21011$

Lee S. J., Lee M. Y., Yi S. Y., Oh S. K., Choi S. H., Her N. H., Choi D., Min B. W., Yang S. G., Harn CH (2002): PPI1: a novel pathogen-induced basic region-leucine zipper (bZIP) transcription factor from pepper. Mol Plant Microbe Interact 15:540-548.

Liu J, Zhu JK (1997): Proline accumulation and salt-stress-induced gene expression in a salthypersensitive mutant of Arabidopsis. Plant Physiol 114: 591-596

Lupas, A. (1996): Coiled coils: new structures and new functions. Trends biochem Sci, 21, 375- 382.

McLachan, A. and Steward, M. (1975): Tropomyosin coiled- coil interactions: evidence for an unstaggered structure. J. Mol. Biol., 98, 293- 304.

Mikami, K., Katsura, M., Ito, T., Okada, K., Shimura, Y. and Iwabuchi, M. (1995): Developmental and tissue-specific regulation of the gene for the wheat basic/leucine zipper protein HBP-1a (17) in transgenic Arabidopsis plants. Mol. Gen. Genet. 248, 573- 582.

Moye-Rowley W. S., Harshman K. D. and Parker C. S. (1989): Yeast YAP1 encodes a novel form of the jun family of transcriptional activator proteins. Genes Dev. 3, 283- 292.

Mönke, G., Altschmied, L., Tewes, A., Reidt, W., Mock, H.P., Bäumlein, H., and 
Conrad, U. (2004): Seed-specific transcription factors ABI3 and FUS3: Molecular interaction with DNA. Planta 219, 158- 166

Naar, A.M., Lemon, B.D. and Tjian, R. (2001): Transcriptional coactivator complexes. Annu. Rev. Biochem. 70, 475-501.

Nakashima K, Satoh R, Kiyosue T, Yamaguchi-Shinozaki K, Shinozaki K (1998): A gene encoding proline dehydrogenase is not only induced by proline and hypoosmolarity, but is also developmentally regulated in the reproductive organs of Arabidopsis. Plant Physiol 118: 1233-1241

Niu, X., Renshaw Gegg, L., Miller, L., Guiltinan, M.J. (1999): Bipartite determinants of DNA- binding specifity of plants basic leucin zipper proteins. Plant Mol. Biol. 41: 1- 13

Onate, L., Vicente Carbajosa, J., Lara, P., Diaz, I., and Carbonero, P. (1999): Barley BLZ2, a seed-specific bZIP protein that interacts with BLZ1 in vivo and activates transcription from the GCN4-like motif of B-hordein promoters in barley endosperm. J. Biol. Chem. 274: 9175-9182.

Onodera, Y., Suzuki, A., Wu, C. Y., Washida, H. and Takaiwa, F. (2001): A rice functional transcriptional activator, RISBZ1, responsible for endospermspecific expression of storage protein genes through GCN4 motif. J Biol Chem 276: 14139-14152

Oono, Y., Seki, M., Nanjo, T., Narusaka, M., Fujita, M., Satoh, R., Satou, M., Sakurai, T., Ishida, J., Akiyama, K., Iida, K., Maruyama, K., Satoh, S., Yamaguchi-Shinozaki, K., and Shinozaki, K. (2003): Monitoring expression profiles of Arabidopsis gene expression during rehydration process after dehydration using ca 7000 fulllength cDNA microarray. Plant J 34, 868-887.

O'Shea, E.K., Rutkowski, R., Stafford, W.F., 3rd, and Kim, P.S. (1989): Preferential heterodimer formation by isolated leucine zippers from fos and jun. Science 245, 646-648.

Oyama, T., Shimura, Y. and Okada, K. (1997): The Arabidopsis HY5 gene encodes a bZIP protein that regulates stimulus- induced development of root and hypocotyls. Genes Dev, 11, 29832995.

\section{Parvanova D, Ivanov S, Konstantinova T, Karanov E, Atanassov A, Tsvetkov T,} Alexieva V, Djilianov D (2004): Transgenic tobacco plants accumulating osmolytes show reduced oxidative damage under freezing stress. Plant Physiol Biochem 42: 57-63

Parcy, F., Valon, C., Raynal, M., Gaubier-Comella, P., Delseny, M., and Giraudat, J. (1994): Regulation of gene expression programs during Arabidopsis seed development: Roles of the ABI3 locus and of endogenous abscisic acid. Plant Cell

Peng Z, Lu Q, Verma DP (1996): Reciprocal regulation of delta 1-pyrroline-5-carboxylate synthetase and proline dehydrogenase genes controls proline levels during and after osmotic stress in plants. Mol Gen Genet 253: 334-341

Polge C, Thomas M (2007): SNF1/AMPK/SnRK1 kinases, global regulators at the heart of energy control. Trends Plant Sci 12:20- 28. 
Pollok, B. A. and Heim, R. (1999): Using GFP in FRET-based applications. Trends in Cell Biology, 9, 57- 60.

Ptashne, M. and Gann, A. (1997): Transcriptional activation by recruitment. Nature, 386, 569577

Pysh, L. D., Aukerman, M. J. and Schmidt, R. J. (1993): OHP1: a maize basic domain/ leucin zipper protein that interacts with opaque2. Plant Cell, 5, 227- 236.

Riechmann, J. L., Heard, J., Martin, G., Reuber, L., Jiang, C., Keddie, J., Adam, L., Pineda, O., Ratcliffe, O. J., Samaha, R. R., Creelman, R., Pilgrim, M., Broun, P., Zhang, J. Z., Ghandehari, D., Sherman, B. K. and Yu, G. (2000): Arabidopsis transcription factors; genome- wide comparative analysis among eukaryotes. Science, 290, 2105- 2110

Rook, F., Gerrits, N., Kortstee, A., van Kampen, M., Borrias, M., Weisbeek, P. and Smeekens, S. (1998): Sucrose-specific signaling represses translation of the Arabidopsis ATB2 bZIP transcription factor gene. Plant J. 15, 253-263.

Rügner, A., Frohnmeyer, H., Nake, C., Wellmer, F., Kircher, S., Scha” fer, E. and Harter, K. (2001): Isolation and characterisation of four novel parsley proteins that interact with the transcriptional regulators CPRF1 and CPRF2. Mol. Genet. Genomics, 265, 964- 976.

Satoh R, Nakashima K, Seki M, Shinozaki K, Yamaguchi-Shinozaki K (2002): ACTCAT, a novel cis-acting element for proline- and hypoosmolarity- responsive expression of the ProDH gene encoding proline dehydrogenase in Arabidopsis. Plant Physiol 130: 709-719

Satoh, R., Fujita, Y., Nakashima, K., Shinozaki, K. and Yamaguchi- Shinozaki, K. (2004): A novel subgroup of bZIP proteins functions as transcriptional activators in hypoosmolarity- responsive expression of the ProDH gene in Arabidopsis. Plant Cell Physiol. 45, 309-317.

Schindler, U., Menkens, A.E., Beckmann, H., Ecker, J.R. and Cashmore, A.R. (1992): Heterodimerization between light-regulated and ubiquitously expressed Arabidopsis GBF bZIP proteins. EMBO J. 11, 1261-1273.

Schulze- Lefert, P., Dangl, J. L., Becker- Andre, M., Hahlbrock, K., and Schulz, W. (1989a): Inducible in vivo DNA footprints define sequences necessary for UV light activation of the parsley chalcone synthase gene. Embo J. 8, 651- 656.

Schütze, K., Harter, K. and Chaban, C. (2008): Post-translational regulation of plant bZIP factors. Trend Plant Sci. 13, 247- 255.

Shimizu, H., Sato, K., Berberich, T., Miyazaki, A., Ozaki, R., Imai, R. and Kusano, 
T. (2005): LIP19, a basic region leucine zipper protein, is a Fos-like molecular switch in the cold signaling of rice plants. Plant Cell Physiol. 46, 1623- 1634.

Siberil, Y., Doireau, P., Gantet, P., (2001): Plant G- box binding factors. Molecular structure and activation mechanism. European Journal of biochemistry FEBS 268: 5655- 5666

Smith, S. M. and Stitt, M. (2007): Coordination of carbon supply and plant growth. Plant Cell Environ. 30:1126-49.

Strathmann, A., Kuhlmann, M., Heinekamp, T. and Dröge- Laser, W. (2001): BZI-1 specifically heterodimerises with the tobacco bZIP transcription factors BZI-2, BZI-3/TBZF and BZI-4, and is functionally involved in flower development. Plant J. 28, 397-408.

Thiel, G., Lietz, M. and Hohl, M. (2004): How mammalian transcriptional repressors work. Eur. J. Biochem. 271, 2855- 2862.

Usadel, B., Blasing, O.E., Gibon, Y., Retzlaff, K., Hohne, M., Gunther, M., and Stitt, M. (2008): Global transcript levels respond to small changes of the carbon status during progressive exhaustion of carbohydrates in Arabidopsis rosettes. Plant Physiol. 146: 1834-1861.

van der Krol, A. R. and Chua, N. H. (1991): The basic domain of plant bZIP- proteins facilitates import of a reporter protein into plant nuclei. Plant Cell, 3, 667- 675.

Varagona, M. J. and Raikhel, N. V. (1994): The basic domain in the bZIP regulatory protein Opaque2 serves two independent functions: DNA binding and nuclear localisation. Plant J. 5, 207214.

Vicente-Carbajosa, J., and Carbonero, P. (2005): Seed maturation: Developing an intrusive phase to accomplish a quiescent state. Int. J. Dev. Biol. 49: 645-651.

Vicente-Carbajosa, J., Moose, S. P., Parsons, R.. L., and Schmidt, R. J.(1997): A maize zinc-finger protein binds the prolamin box in zein gene promoters and interacts with the basic leucine zipper transcriptional activator Opaque2. Plant Biology 94, 7685-7690.

Vicente-Carbajosa, J., Onate, L., Lara, P., Diaz, I. and Carbonero, P. (1998): Barley BLZ1: a bZIP transcriptional activator that interacts with endosperm-specific gene promoters. Plant J. 13, 629-640.

Vinson, C., Mayakishev, M., Acharya, A., Mir, AA., Mol, JR., Bonovich, M., (2002): Classification of human bZIP- proteins based on dimerisation properties. Mol. Cell. Biol. 22: 6321- 6335

Verbrüggen N., Hua X.J., May M., Van Montagu M. (1996): Environmental and developmental signals modulate proline homeostasis: evidence for a negative transcriptional regulator. Proc Natl Acad Sci USA 93: 8787-8791

Vogt, P.K., Bos, T.J. and Doolittle R.F. (1987): Homology between the DNA binding domain 
of the GCN4 regulatory protein of yeast and the carboxy-terminal region of a protein encoded for by the oncogene jun. Proc. Natl. Acad. Sci. 84: 3316-3319.

Walter, M., Chaban, C., Schütze, K. (2004): Visualization of protein interactions in living plant cells using bimolecular fluorescence complementation. Plant J. 40, 428-438.

Weltmeier F, Ehlert A, Mayer CS, Dietrich K, Wang X, Schütze K, Harter K, Vicente- Carbajosa J, Dröge- Laser W (2006): Combinatorial control of Arabidopsis proline dehydrogenase transcription by specific heterodimerisation bZIP transcription factors. EMBO J 25:3133-3143.

Weltmeier, F., Rahmani, F., Ehlert, A., Dietrich, K., Schütze, K., Wang, X., Chaban, C., Hanson, J., Teige, M., Harter, K., Vicente-Carbajosa, J., Smeekens, S., and Dröge-Laser, W. (2009): Expression patterns within the Arabidopsis C/S1 bZIP transcription factor network: availability of heterodimerization partners controls gene expression during stress response and development. Plant Mol Biol 69, 107-119.

Wiese A, Elzinga N, Wobbes B, Smeekens S (2004): A conserved upstream open reading frame mediates sucrose-induced repression of translation. Plant Cell 16:1717-1729.

Wobus, U. and Weber, H. (1999): Sugar as signal molecules in plant seed development. Curr. Opin. Plant Biol. 2, 33- 38.

Yang, J. C., Zhang, J. H., Wang, Z. Q., Zhu, Q. S. and Liu, L. J. (2003): Involvement of abscisic acid and cytokinins in the senescence and remobilization of carbon reserves in wheat subjected to water stress during grain filling. Plant Cell Environ, 26, 1621- 1631.

Yoshiba Y, Nanjo T, Miura S, Yamaguchi-Shinozaki K, Shinozaki K (1999): Stressresponsive and developmental regulation of Delta (1)-pyrroline-5-carboxylate synthetase 1 (P5CS1) gene expression in Arabidopsis thaliana. Biochem Biophys Res Commun 261: 766-772

Zimmermann, P., Hirsch-Hoffmann, M., Hennig, L. and Gruissem W. (2004): GENEVESTIGATOR. Arabidopsis Microarray Database and Analysis Toolbox. Plant Physiology, 136, 2621- 2632,

Zuo J, Niu QW, Chua NH (2000): Technical advance: an estrogen receptor-based transactivator XVE mediates highly inducible gene expression in transgenic plants. Plant J 24: 265-273 


\section{Contibutions}

Paper:

"Two-hybrid protein-protein interaction analysis in Arabidopsis protoplasts: establishment of a heterodimerization map of group C and group S bZIP transcription factors" Ehlert and Weltmeier et al., 2006 Plant Journal

Figure:

- Figure 2. Activation properties of BD-AtbZIP proteins in yeast.

- Figure 3. Heterodimerization analysis of groups $\mathrm{C}$ and S1 bZIP proteins using a yeast two-hybrid assay.

- Figure 4. Heterodimerization of group S2 bZIP protein using a yeast two hybrid assay.

- Figure 5. Development of a protoplast based two-hybrid system (P2H) in Arabidopsis.

- Figure 6. Heterodimerization of groups $\mathrm{C}$ and S1 bZIP proteins using a $\mathrm{P} 2 \mathrm{H}$ assay system in A. thaliana protoplasts.

- Table 1. Interaction pattern of group C and group S AtbZIP transcription factors obtained by yeast twohybrid analysis

- Table 2. Interaction pattern of group C and group S1 AtbZIP transcription factors obtained by P2H analysis.

Paper:

"Combinatorial control of Arabidopsis proline dehydrogenase transcription by specific heterodimerisation of bZIP transcription factors"

Weltmeier and Ehlert et al., 2006 Embo Journal

Figure:

- Figure 3. Yeast two-hybrid analysis studying heterodimerisation of AtbZIP53 with group-S and -C bZIP factors.

- Figure 5. Analysis of AtbZIP53 heterodimerisation in transiently transformed Arabidopsis protoplasts.

- Figure 6. ACTCAT-specific DNA binding of group-S1 and C bZIP factors.

- Figure 7. The ACTCAT-mediated transcription is regulated by AtbZIP53 heterodimers.

- Figure 8. The ACTCAT-mediated transcription in Arabidopsis protoplasts depends on AtbZIP53/AtbZIP10 heterodimers.

- Supplementary Table S1: Heterodimer Induced Transactivation (HIT) by group-C / S1 heterodimers. 
Paper:

"Expression patterns within the Arabidopsis C/S1 bZIP transcription factor network: availability of heterodimerisation partners controls gene expression during stress response and development"

Weltmeier et al., 2009 Plant Mol Biol

Figure:

- Figure 2. Regulation of the $2 \mathrm{~S}$ albumin (2S2) seed storage protein promoter by group C and group S1 bZIP TFs in transiently transformed Arabidopsis leaf mesophyll protoplasts.

Paper:

"A Pivotal Role of the Basic Leucine Zipper Transcription Factor bZIP53 in the Regulation of Arabidopsis Seed Maturation Gene Expression Based on Heterodimerization and Protein Complex Formation"

Alonso et al., 2009 Plant Cell

Figure:

- Figure 4. D Interaction of bZIP53, bZIP10, and bZIP25 Homo- or Heterodimers with ABI3.

- Figure 6. Effect of ABI3 on the Transcriptional Activation Mediated by the bZIP53/bZIP10 Heterodimers.

- Suplemental Figure 2. Transient expression of bZIP53 in combination with bZIP10 or bZIP25 synergistically enhances transactivation of CRU3 and $2 S 2$ promoters.

Paper:

"Heterodimers of Arabidopsis transcription factors bZIP1 and bZIP53 are reprogramming amino acid metabolism during low energy stress"

Dietrich et al., submitted

Figure:

- Figure 9. bZIP1 binds directly to the ASN1 promoter and mediates starvation responses via a G-box ciselement.

- Supplementary Figure S9. Mutation in the C-box effects ProDH promoter activity. 
Paper:

"Gene expression analysis reveals differential functions of bZIP dimmers in protoplasts" Hanssen et al., unpublished

Figure:

- tansfection of Arabidopsis mesophyll protoplasts with DNA encoding AtbZIP11, AtbZIP10 and both, for global gene expression analysis

- Figure 4.1. bZIPs are highly expressed in transfected protoplasts

Heterodimers of Arabidopsis transcription factors bZIP1 and bZIP53 are reprogramming amino acid metabolism during low energy stress 


\section{Supplementary}

Supplemental Data: WELTMEIER et al., (2009):

"Expression patterns within the Arabidopsis C/S1 bZIP transcription factor network: availability of heterodimerisation partners controls gene expression during stress response and development”

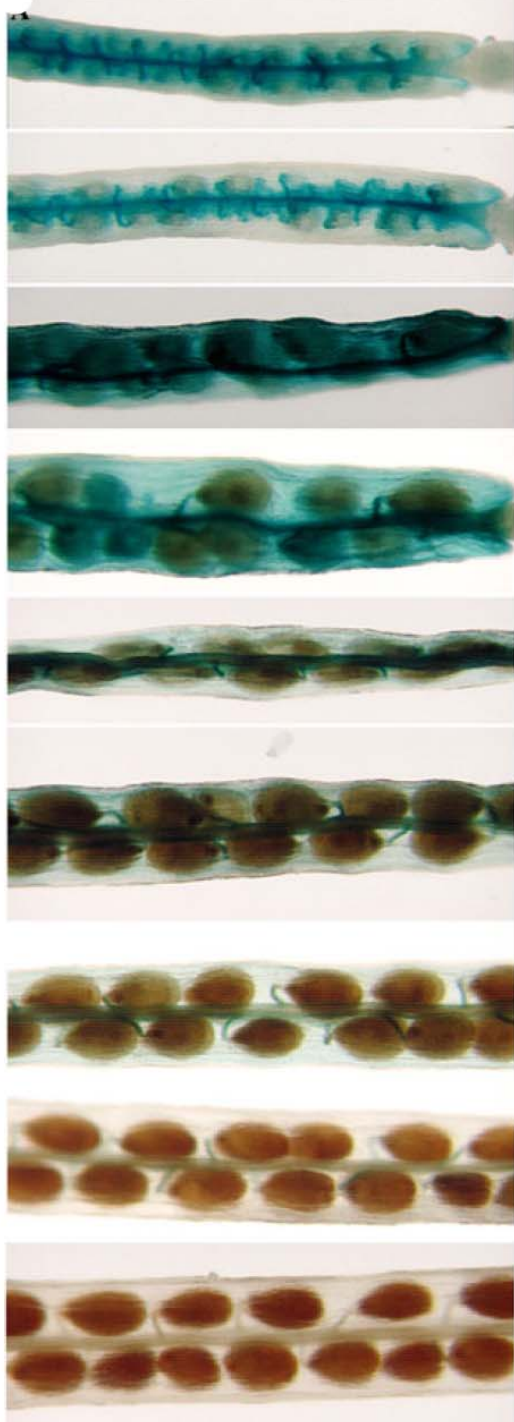

AtbZIP11 /ATB2
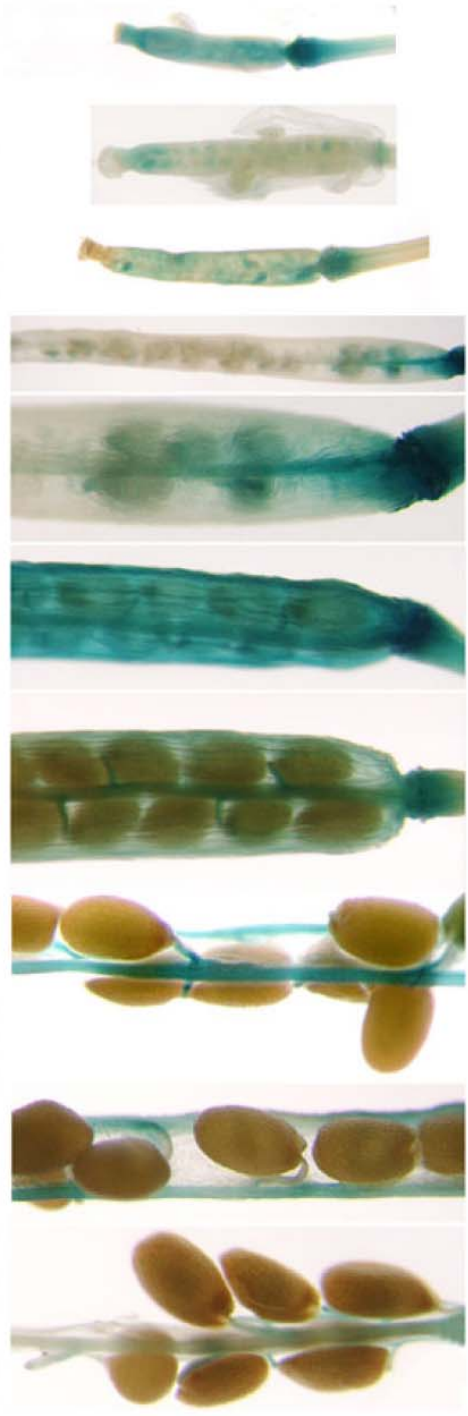

AtbZIP44


AtbZIP2

Supplementary Fig. 1 Histochemical staining of $\operatorname{Pro}_{A t b Z I P}$ :GUS lines analysing expression of AtbZIP11, AtbZIP2 and AtbZIP44 during seed development. Given are siliques with seeds of increasing developmental age (from top to bottom). 


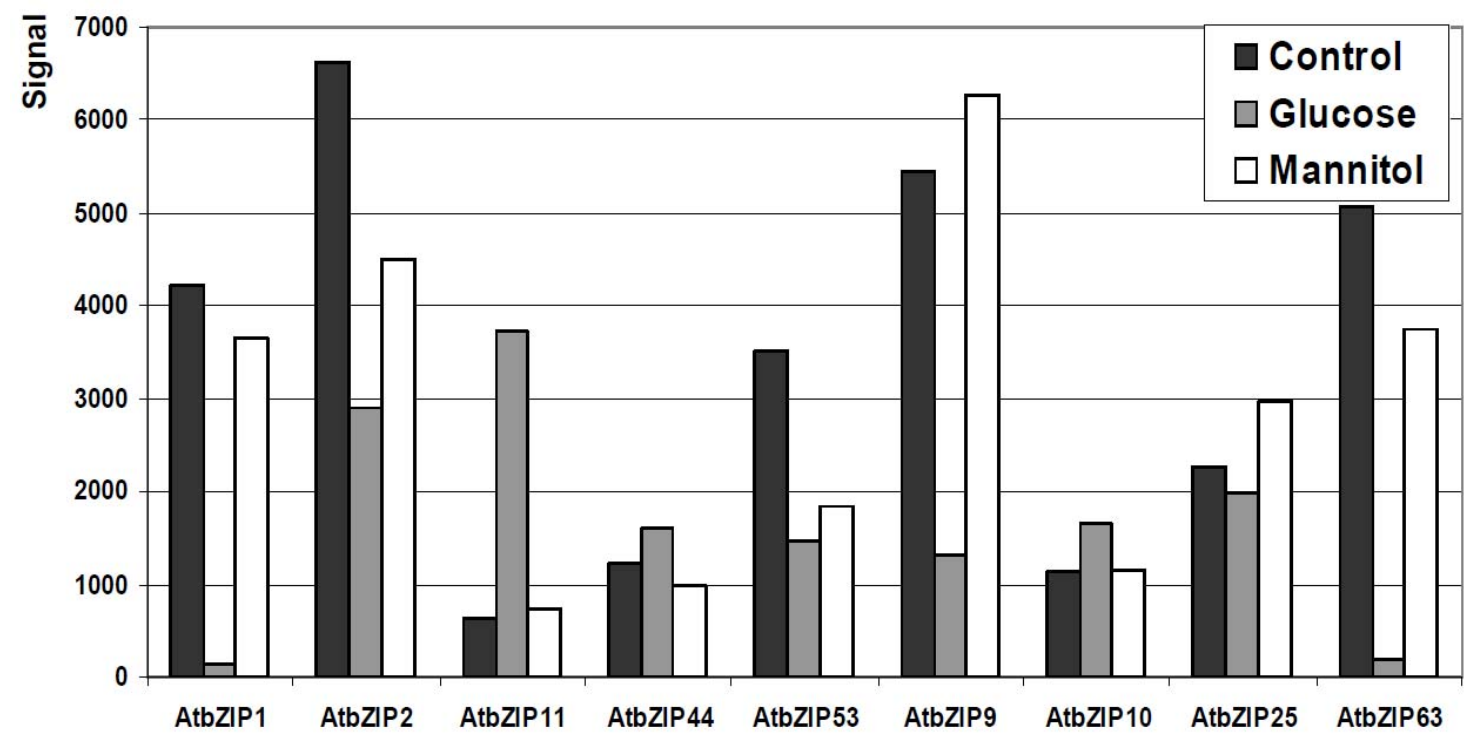

Supplementary Fig. 2 Regulation of group C and S1 AtbZIP genes by glucose as described in public array data sets (ZIMMERMANN et al., 2004). Arabidopsis plantlets were grown in liquid culture for 7 days on MS medium containing $0.5 \%$ glucose in constant light. After 7 days of growth, the medium was replaced with glucose-free medium for $24 \mathrm{~h}$. Afterwards plants were transferred for $2 \mathrm{~h}$ into $3 \%$ glucose or 3\% mannitol, respectively. Mannitol serves as an osmotic control.

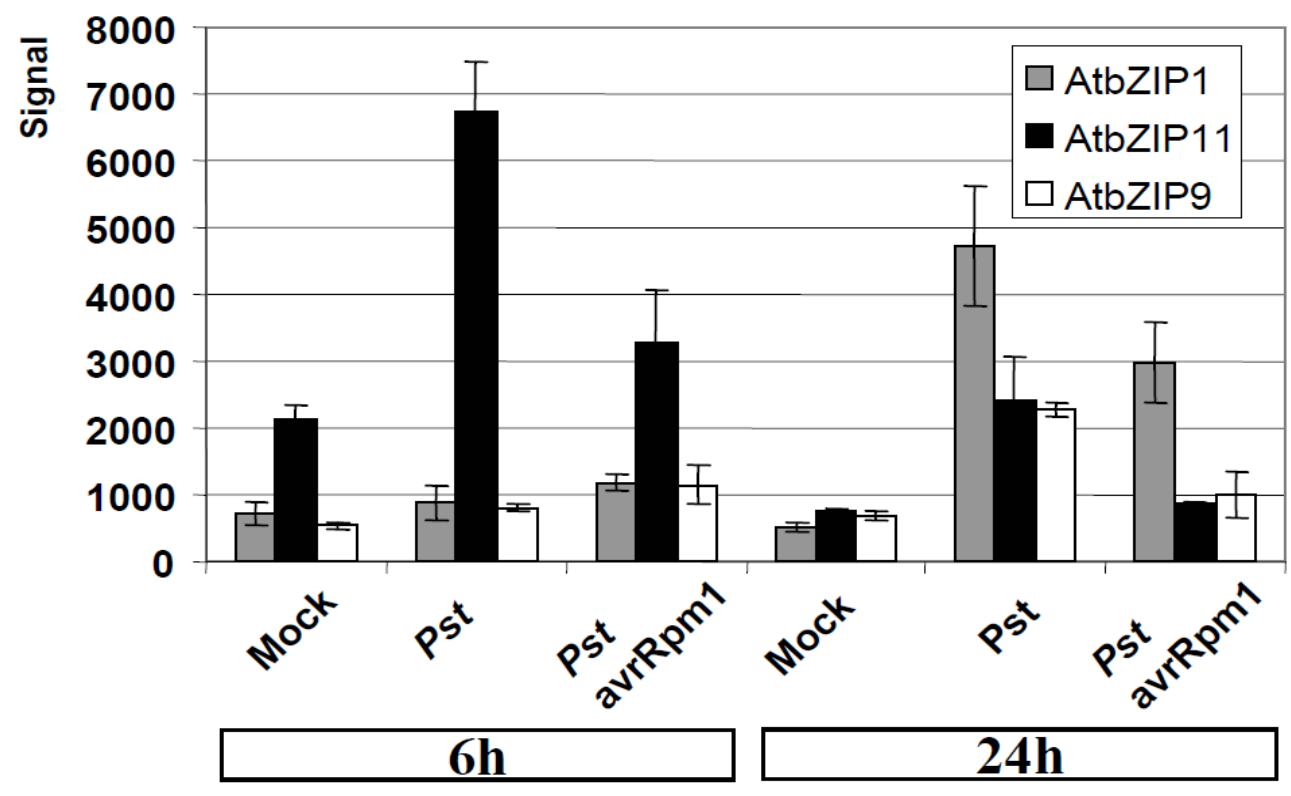

Supplementary Fig. 3 Induction of AtbZIP1 and AtbZIP11 in response to Pseudomonas syringae infection. Given are relative signals of micro array results from Arabidopsis plants, $6 \mathrm{~h}$ or $24 \mathrm{~h}$ post infection (ZIMMERMANN et al., 2004). Pst: Pseudomonas syringae pv. tomato, Pst avrRpm1: Pseudomonas syringae pv. tomato carrying an avrRpm1 avirulence gene. Mock treatment was performed by infiltration of $\mathrm{MgCl}_{2}$ solution. 


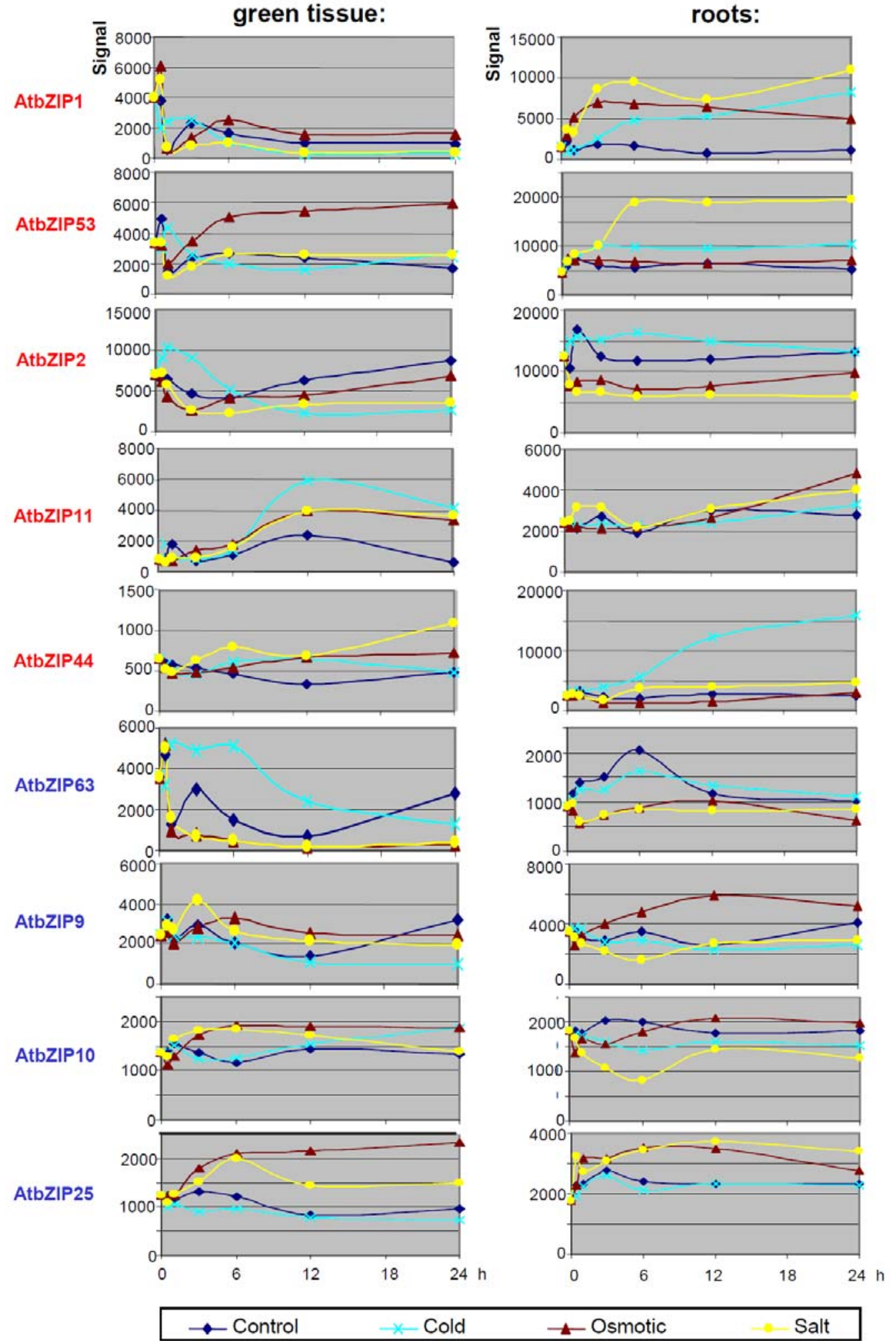

Supplementary Fig. 4 Transcript abundance of group S1 and group C AtbZIP genes in response to abiotic stresses. The diagrams are based on AtGenExpress profiles (KILIAN et al. 2007). For each time point the average value of three biological replicates is shown. Treatments are indicated (control: dark blue; cold: light blue; osmotic stress: brown; salt stress: yellow). Compared are green tissues (left) and roots (right). 
Supplementary Tab. 1 List of primers used for PCR amplification

\begin{tabular}{|c|c|c|}
\hline Gene & Used for & Primers \\
\hline \multirow[t]{2}{*}{ AtbZIP1 } & UBQ :leader: GUS & F:5'-GCGAATTCAAATTCTCTTTCTTTTCCGATTCGTC-3' \\
\hline & & R:5'-CGCCATGGCCATGTTTTTTTGTTCAAGAGATAA-3' \\
\hline \multirow[t]{2}{*}{ AtbZIP2 } & UBQ :leader: GUS & F:5'-GCGAATTCTTCCCATTCACTCATCTTCTCTCTTCC-3' \\
\hline & & R:5'-GCCCATGGCTGCTAGATGACGCCATTGATT-3' \\
\hline \multirow[t]{2}{*}{ AtbZIP11 } & UBQ :leader: GUS & F:5'-GCGAATTCTTCATTTCTTAGAGATCTCAGCTTC-3' \\
\hline & & R:5'-GCCCATGGCGACGATTCCATTTAGTAACACAC-3' \\
\hline \multirow[t]{3}{*}{ AtbZIP44 } & UBQ :leader: GUS & F:5'-GCGAATTCCCTCACAAATCCTTAATTAATTCACC \\
\hline & & TCTTC-3 \\
\hline & & R:5'-GCCCATGGCCATTTCAGTTTTATTATTCATATTA-3' \\
\hline \multirow[t]{2}{*}{ AtbZIP53 } & UBQ :leader: GUS & F:5'-GCCTGCAGGCTTTTTTTCGTTTATCAATCTTCTCG-3' \\
\hline & & R:5'-CGCCATGGCCATTTCTCGTTGACTTTTTGACTTC-3' \\
\hline \multirow[t]{3}{*}{ UBQ10 } & UBQ :leader: GUS & F:5'-GAGCGGCCGCGATCAGGATATTCTTGTTTAAGAT \\
\hline & & GTT-3' \\
\hline & & R:5'- GCCTGCAGTTTATAGAAGAAGAAGAAGAAA-3' \\
\hline \multirow[t]{2}{*}{ AtbZIP1 } & Promoter: GUS & F:5'-GTCGACACCACAATTTGGAACTAATA-3' \\
\hline & & R:5'- GAATTCTCAAGAGATAACGTAAAACC-3' \\
\hline \multirow[t]{2}{*}{ AtbZIP53 } & Promoter: GUS & F:5'-CTGCAGAAGTCCAGGTCAAGTTTGTT-3' \\
\hline & & R:5'- GTCGACCTCGTTGACTTTTTGACTTC-3' \\
\hline \multirow[t]{2}{*}{ AtbZIP9 } & Promoter: GUS & F:5'- -3' GTCGACCAAACAGGTTATGTAGTAGA \\
\hline & & R:5'- -3' TCTAGAAGCTGTGTGATTATCCAT \\
\hline \multirow[t]{2}{*}{ AtbZIP63 } & Promoter: GUS & F:5'- -3' GGATCCACTGCGATTCAACGACG \\
\hline & & R:5'- -3' GTCGACAGAGGACGTTGGTGATAG \\
\hline \multirow[t]{2}{*}{ AtbZIP53 } & hybridisation & F:5'- CATACAAAGACACGAACAAATTGAA -3' \\
\hline & & R:5'- GTGCTTGAGATGGTTGAAGAAATTA -3' \\
\hline \multirow[t]{2}{*}{ AtbZIP1 } & hybridisation & F:5'- GGCAAACGCAGAGAAGACAA -3' \\
\hline & & R:5'- GGACGCCATTGGTTGTAGAG -3' \\
\hline \multirow[t]{2}{*}{ AtbZIP9 } & hybridisation & F:5'- CGCAACACAACAGTTTCGTA -3' \\
\hline & & R:5'- GCCAGATGTCTGAGACGCAG -3' \\
\hline \multirow[t]{2}{*}{ AtbZIP63 } & hybridisation & F:5'- GCGTGTGGTGTTTCCGTCTC -3' \\
\hline & & R:5'- CTACTGATCCCCAACGCTTC -3' \\
\hline
\end{tabular}


Supplemental Data: WETMEIER et al., (2006):

\section{“Combinatorial control of Arabidopsis proline dehydrogenase}

\section{transcription by specific heterodimerisation of bZIP transcription}

\section{factors”}

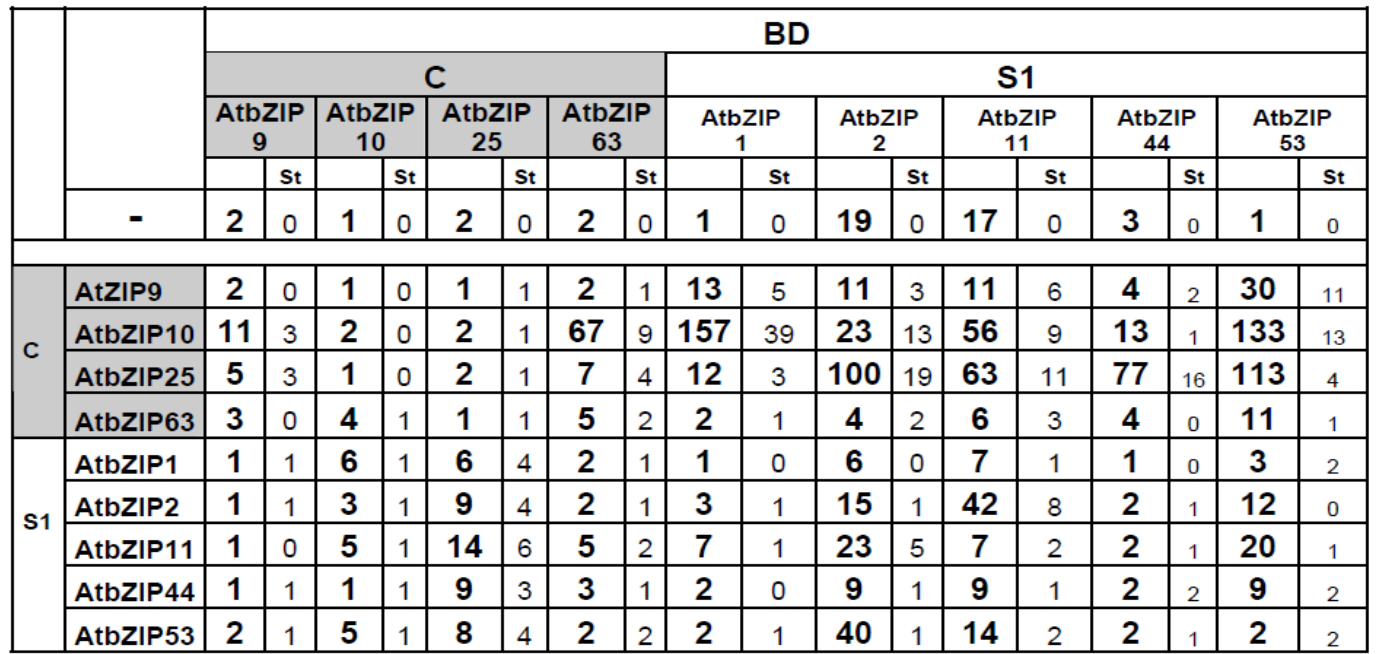

Table S1 Heterodimer Induced Transactivation (HIT) by group-C / S1 heterodimers.

Activation of a GAL-UAS4:GUS reporter in Arabidopsis protoplasts after expression of BD-AtbZIP fusion proteins without (-) and with an AtbZIP heterodimerisation partners (AtbZIP). Given are mean values and standard deviations ( $\mathrm{St}$ ) of four independent transfections as relative GUS / NAN units. In comparison to the gene activation mediated by the BD-bZIP proteins, the HIT mechanism leads to strong activation.

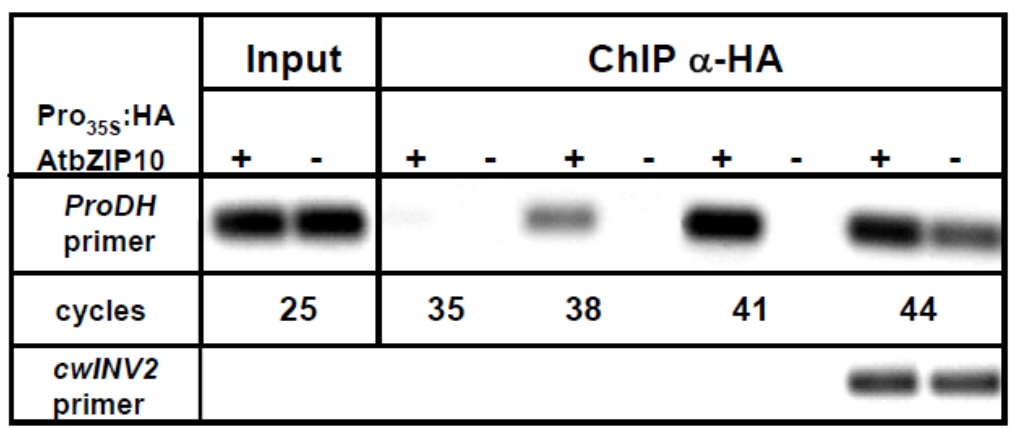

Figure S1. In vivo binding of AtbZIP10 to the ProDH promoter. ChIP analysis of chromatin obtained from Pro35S:HA-AtbZIP10 (+) and Wt (-) plants using a HA-specific antibody for immunoprecipitation. ProDH primers were used to amplify a $191 \mathrm{bp}$ fragment of the ProDH promoter. Given are the PCR products obtained after 35, 38, 41 or 44 cycles, respectively. As input control, PCR amplicons are shown which have been obtained after 25 cycles with material before immunoprecipitation. The specificity was verified by using primers amplifying the promoter of a nontarget gene (cwINV2, At3g52600). Given are amplicons obtained after 35, 38, 41 or 44 cycles, respectively. The PCR products were stained by ethidium bromide. 
A

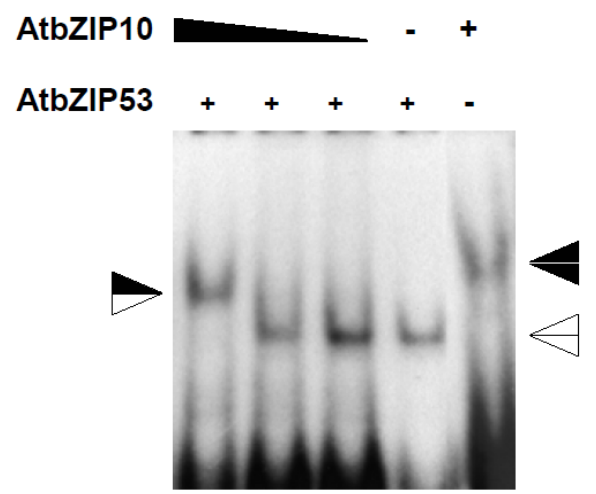

B

\section{AtbZIP}

$10 \quad 53$

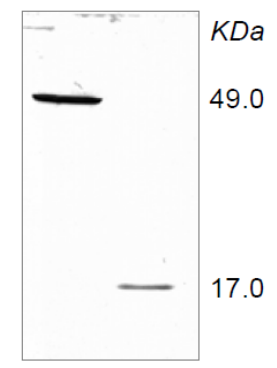

Figure S2 AtbZIP10 and AtbZIP53 bind DNA as homo or heterodimers.

A Electrophoretic mobility shift assay using a labelled dsDNA probe derived from the At ProDH promoter (5'-ATTCATCATCC ACTCAT CCTTCATATCT- 3') and His-tag proteins AtbZIP53 and AtbZIP10 expressed in E.coli. The black and white arrows indicate the retarded bands with different mobility produced by AtbZIP10 and AtbZIP53 homodimers, respectively. When increasing amounts of AtbZIP10 protein were added to the AtbZIP53 binding reaction a band of intermediate mobility corresponding to the heterodimer was obtained (combined black and white arrow).

B Westernblot detection of proteins used in the gel retardation experiments. A poly- His antibody was used to detect of E.coli produced proteins AtbZIP10 and AtbZIP53 with a His-tag epitope.

\section{$\begin{array}{lllll}0 & 0.5 & 1 & 2 & h\end{array}$}

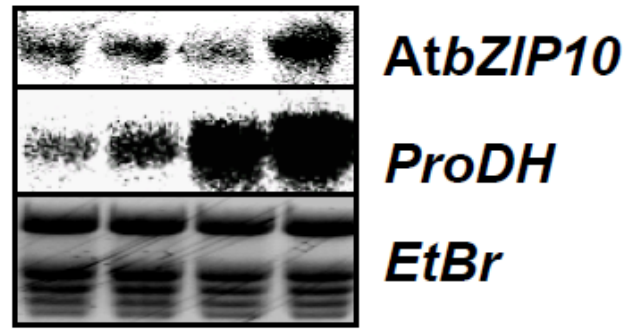

Figure S3. Gene induction by hypoosmotic stress treatment.

14 day-old Arabidopsis plants were transferred from MS medium (58 mM sucrose) to desalted water (Satoh et al., 2004). RNA of control $(0 \mathrm{~h})$ and stress treated plants (time points as indicated) was analysed by Northern hybridisation. Depicted are hybridisation experiments with ProDH and AtbZIP10 specific probes, respectively. Equal loading was verified by ethidium bromide (EtBr) staining. 
Supplementary Data 5;

Methods S1:

\section{ChIP}

Soil grown plants (typically 5-7 g) were vacuum infiltrated in $50 \mathrm{mM}$ potassium phosphate, $\mathrm{pH}$ $7.5,1 \%$ formaldehyde for $30 \mathrm{~min}$, then cross-linking was stopped by vacuum infiltration in $50 \mathrm{mM}$ potassium phosphate, $\mathrm{pH}$ 7.5, $300 \mathrm{mM}$ glycine for $15 \mathrm{~min}$. Plant material was washed twice with desalted water and then powdered with a mortar and pestle under liquid nitrogen. The following steps were performed at $4^{\circ} \mathrm{C}$. Tissue powder was resuspended in $20 \mathrm{ml}$ extraction buffer $(1 \mathrm{M}$ hexylenglycol, $50 \mathrm{mM}$ PIPES-KOH pH 7.2, $10 \mathrm{mM} \mathrm{MgCl}_{2}, 5 \mathrm{mM}$ ß-mercapto-ethanol) and filtered through miracloth. $25 \%$ Triton X-100 was slowly added to a final concentration of $1 \%$, and the suspension was incubated under gentle stirring for 15-30 min. The suspension was overlaid on $6 \mathrm{ml}$ 75\% Percoll buffer (75\% Percoll, 0,5 M hexylenglycol, $50 \mathrm{mM}$ PIPES-KOH pH 7.2, $10 \mathrm{mM}$ $\mathrm{MgCl}_{2} 1 \%$ Triton X-100, $5 \mathrm{mM}$ ß-mercapto-ethanol) and centrifuged at $4^{\circ} \mathrm{C} / 2000 \mathrm{~g}$ for $30 \mathrm{~min}$. The pellet was resuspended in $20 \mathrm{ml}$ gradient buffer $(0.5 \mathrm{M}$ hexylenglycol, $50 \mathrm{mM}$ PIPES-KOH $\mathrm{pH} 7.2,10 \mathrm{mM} \mathrm{MgCl} 2,1 \%$ Triton X-100, $5 \mathrm{mM}$ ß-mercaptoethanol) and overlaying on / centrifugation through $6 \mathrm{ml} 75 \%$ Percoll buffer was repeated.

The crude nuclear pellet was resuspended in $500 \mu \mathrm{l}$ of TE / 0,5\% SDS, incubated for 20 min with gentle mixing at $4^{\circ} \mathrm{C}, 500 \mu \mathrm{TE}(\mathrm{pH} 8)$ were added and chromatin was solubilised with a probe sonicator (Soniprep 150, Scientific Instruments) with 4-10 s pulses at 12 micron and extensive cooling between pulses. Insoluble material was pelleted $\left(12000 \mathrm{~g}, 5 \mathrm{~min}, 4^{\circ} \mathrm{C}\right)$ and the chromatin was aliquoted in $200 \mu \mathrm{l}$ fractions. $100 \mu \mathrm{l}$ of chromatin solution were decrosslinked to determine the DNA concentration and to check its size (ideally under $1 \mathrm{~kb}$ ).

Equal amounts of chromatin were brought to the same volume $(200 \mu 1)$ and pre-adsorbed with protein A-Sepharose (Sigma-Aldrich Co., Taufkirchen, Germany) for $1 \mathrm{~h}$ at $4^{\circ} \mathrm{C}$ on a rotator. After centrifuging in a micro-centrifuge at top speed, $2 \mathrm{~min}, 4^{\circ} \mathrm{C}$, the supernatant was moved to a new tube and $6 \mu$ anti-HA-antibody (Santa Cruz, Santa Cruz, Ca, USA) were added. After incubation at $4^{\circ} \mathrm{C}$ with gentle mixing for $4 \mathrm{~h}, 30 \mu 1$ protein A-Sepharose (50\% slurry) were added and incubation was continued for another hour. Protein A-Sepharose beads were pelleted in a micro-centrifuge at top speed for $1 \mathrm{~min}$. the supernatant was removed and analysed by protein gel blot as 'post-bind' fraction. The beads were washed 4 times for 5 min with 1ml RIPAF (50 mM HEPES pH 7.4, 140 $\mathrm{mM} \mathrm{NaCl}, 1 \mathrm{mM}$ EDTA, $1 \%$ Triton $\mathrm{x}-100,0.1 \%$ DOC, $0.1 \%$ SDS) on a rotator. The beads and IP buffer were moved to a new tube at the last wash. The beads were pelleted, wash removed, and protein was eluted with $200 \mu \mathrm{l}$ glycine elution buffer $(100 \mathrm{mM}$ glycine, $500 \mathrm{mM} \mathrm{NaCl}, 0.05 \%$ 
Tween 20) for $30 \mathrm{~s}$. The beads were pelleted (room temperature, $1 \mathrm{~min}$, top speed), and the supernatant was removed to a tube with $50 \mathrm{ml}$ of $1 \mathrm{M}$ Tris, $\mathrm{pH} 9$ to neutralize the eluat. A part of the eluat was analysed on a protein gel blot to assess recovery of precipitated HA-AtbZIP53.

The eluat was decrosslinked overnight at $65^{\circ} \mathrm{C}$, RNase A / proteinase $\mathrm{K}$ treated, and DNA was recovered by phenol / chloroform extraction. Recovered DNA was analysed by PCR with ProDH promoter primers CAAGTCCAGGTCCACATGTTG (PDHpromfor)

CAAGGCCCTGACCATAGGA (PDHpromrev) and unspecific control primers GAAGGGTTGGGCCGGAAT (AtcwINV2for) / TTGCGAAGCTTTCGTCAAAC (AtcwINV2rev) of the invertase gene (At3g52600).

\section{Vector construction}

Gateway entry vectors (pDONR201, pDONR207; Invitrogen, Karlsruhe, Germany) containing the different Arabidopsis group-S and group-C bZIP genes were available by the REGIA project (JAKOBY et al., 2002). Point mutations were created in the pDONR207-AtbZIP53 plasmid with the QuikChange Multi Site-Directed mutagenesis kit (Stratagene, La Jolla, Ca, USA) as described by the manufacturer (Invitrogen, Karlsruhe, Germany). Primers used for mutagenesis were

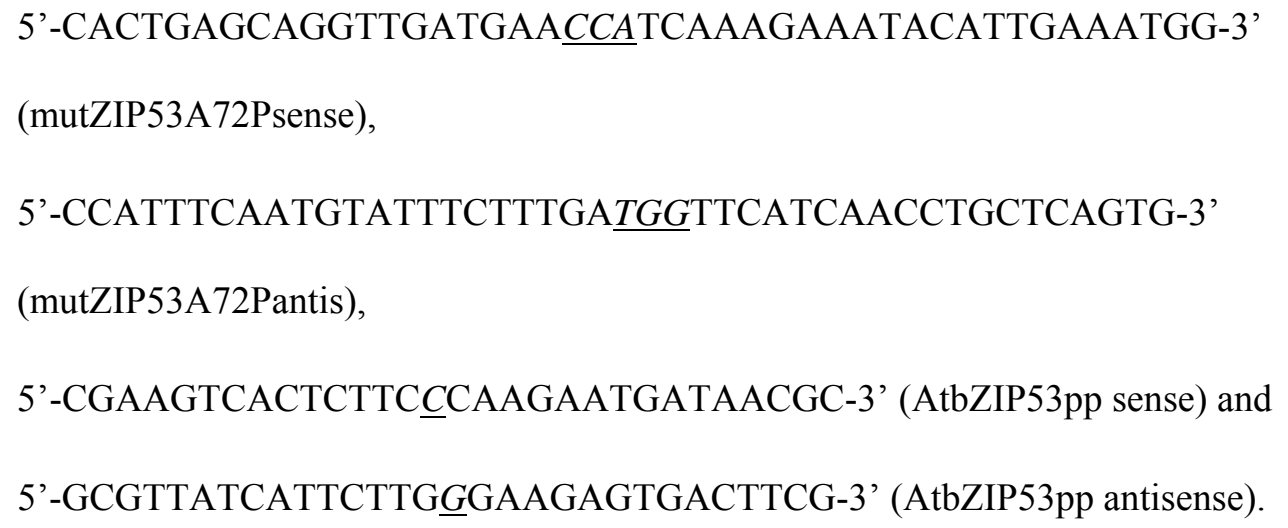

From the entry vectors the AtbZIP genes were recombined into the ProQuest ${ }^{\circledR}$ Y2H Vectors

pDEST22 and pDEST32 (INVITROGEN, Karlsruhe, Germany) and the plant transformation vector pAlligator2 (BENSMIHEN et al., 2004), p35S-GAD-GW, p35S-BDGW, and p35S-HA-GW (EHLERT et al., 2006).

For construction of $(\mathrm{ACTCAT})_{2}: \mathrm{GUS}$ and $(\mathrm{ACTttg})_{2}: \mathrm{GUS}$ reporter constructs, the oligonucleotides $\operatorname{ProDH}_{\text {as }}\left(5^{\prime}\right.$-CTAGAAGATATGAAGGATGAGTGGATGATGAATCACTAGTG-3') 
ProDH $_{\text {asmut }}\left(5^{\prime}\right.$-CTAGAAGATATGAAGGATGtaaGGATGATGAATCACTAGTG-3')

ProDH $_{\text {smut }} \quad$ (5'-AATTCACTAGTGATTCATCATCCttaCATCCTTCATATCTT-3') were ligated into EcoRI and XbaI restriction sites in the vector pBT10-GUS (Sprenger-Haussels, Weisshaar, 2000).

A 1549 bp AtbZIP53 promoter fragment was PCR amplified using the primers bZIP53pGUSf2 (5'-CTGCAGAAGTCCAGGTCAAGTTTGTT-3') and bZIP53pGUSr $\quad$ (5'GTCGACCTCGTTGACTTTTTGACTTC-3') and inserted in pGEM-T-vector (Promega, Mannheim, Germany). The promoter was isolated by PstI/SalI restriction and ligated into the pCAMBIA-1391Z plant transformation vector (AF234312). 
Supplemental Data: DIETRICH et al., (unpublished):

"The Arabidopsis C/S1 bZIP transcription factor network is reprogramming amino acid metabolism in response to low energy stress "

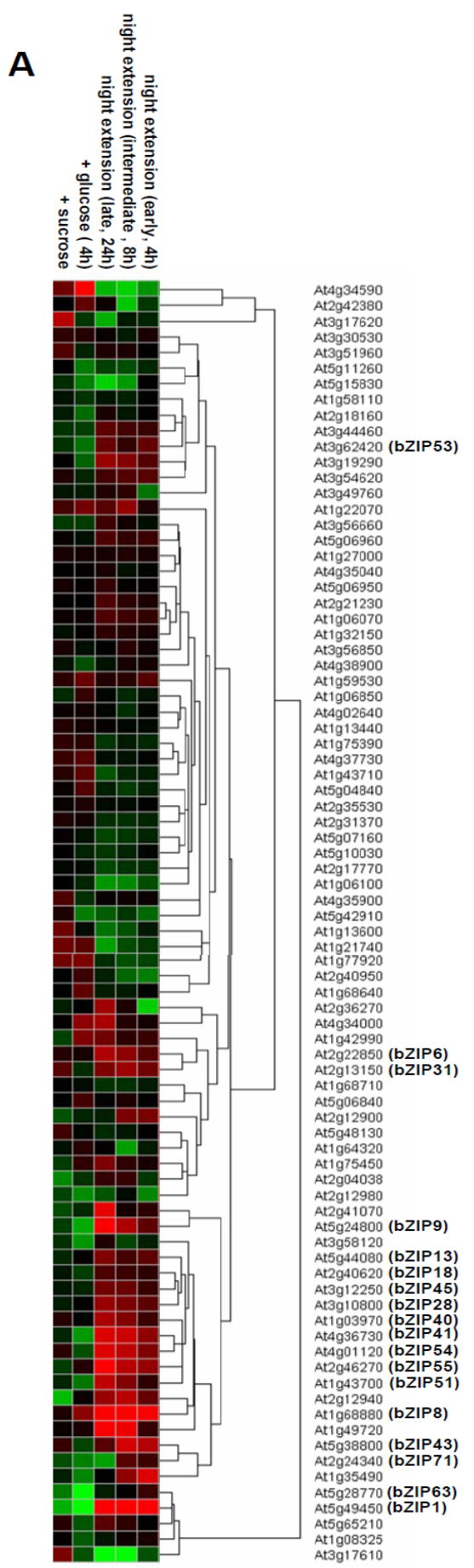

B

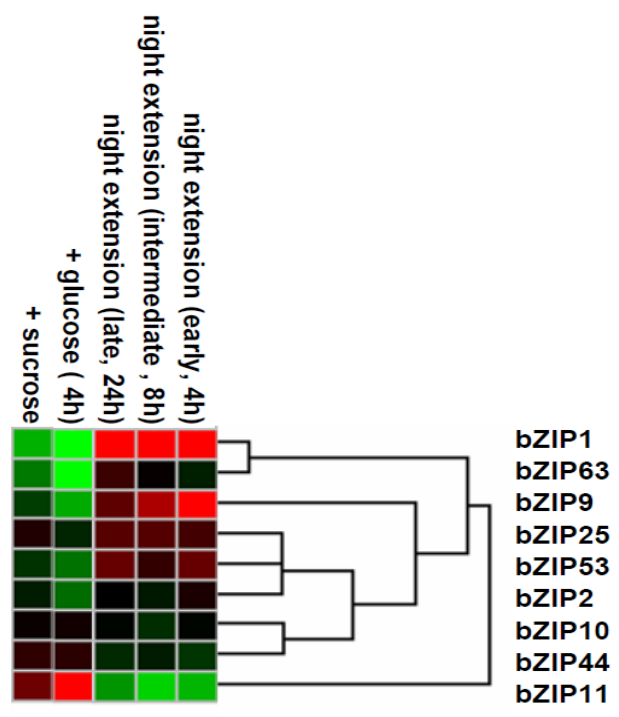

C

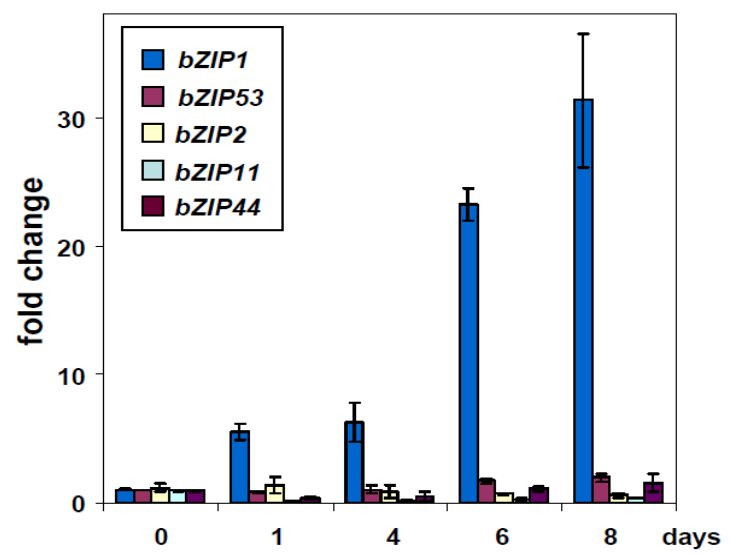




\section{Supplemental S1. Expression of bZIP genes during energy starvation.}

A Cluster analysis of all Arabidopsis bZIP genes according to JAKOBY et al. (2002) making use of public expression data and the Genevestigator tool (Zimmermann et al., 2004). Transcriptome data sets analysing energy deprivation are compared with respect to early, intermediate and late night extension, respectively (time points as indicated). Opposed are treatments with glucose or sucrose (for $4 \mathrm{~h}$ ). Red and green colours indicate induction or repression of the corresponding genes.

B Cluster analysis for the nine group S1 and C bZIP TFs (Ehlert et al., 2006) analysing the treatments described in A.

C Transcript abundance of the group S1 bZIP TF genes after $0-8$ days of dark treatment measured by qPCR analysis. Labelling of the bars are indicated. Given are fold change levels relative to the " 0 " time point of the wt. Error bars represent mean values and standard deviation of 2 repetitions.

A

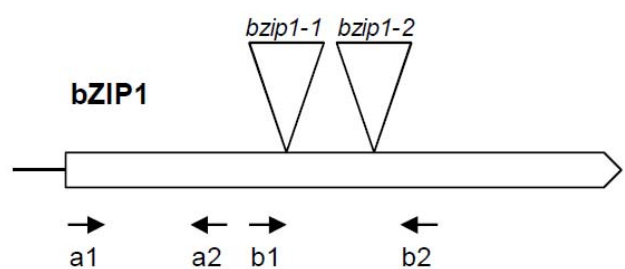

B
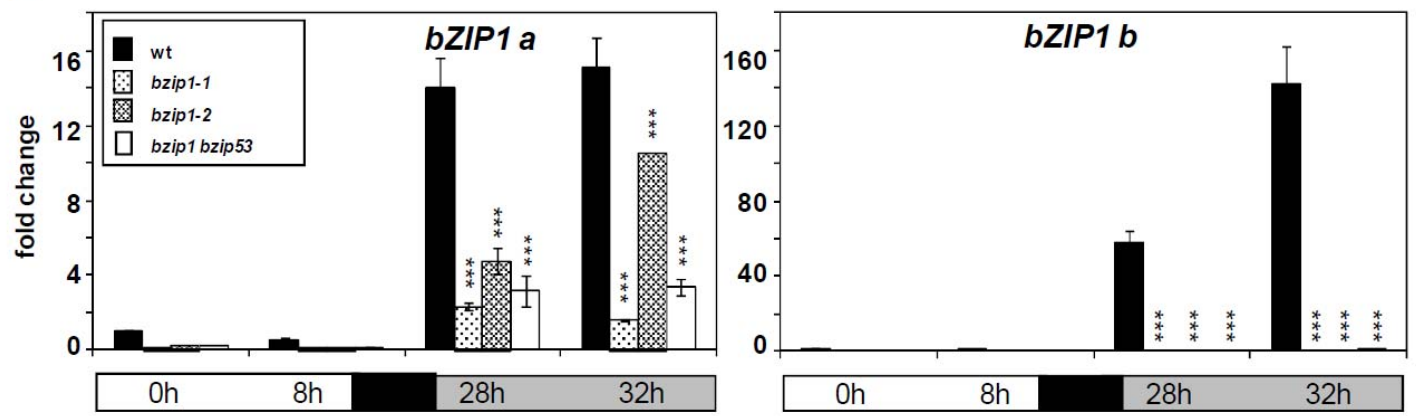

C

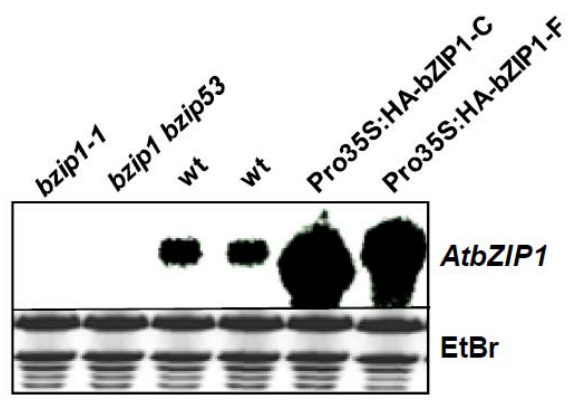

D

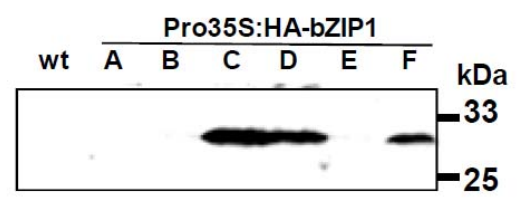

Supplemental Figure S2. Molecular characterisation of transgenic plants altering the amount of bZIP1.

(A)Schematic view showing location of the T-DNA insertion in the SALK lines bzip1-1 (SALK_059343) and bzip1-2 (SALK_069489). Given are the primers used for the mutant characterisation provided in (B) which amplify fragments in the 5' (a1 and a2) or a 3' (b1 and b2) region of the gene. (B) Expression analysis of bZIP1 in the T-DNA mutant lines bzip1-1, bzip1-2 and the double mutant bzip1 bzip53 performed by qPCR using primer pair a (bZIP1a) or b (bZIP1b). For the double and quadruple mutants, the bzip1-1 allel has been used. The light/dark regime is given in the schematic drawing. Depicted is the fold change relative to wt expression at $0 \mathrm{~h}$. Asterisks represent significant differences of mutant plants compared to the wt at the indicated time point (twoway ANOVA, $* \mathrm{p}<0.05 ; * * \mathrm{p}<0.01 ; * * * \mathrm{p}<0.001$ ). (C) Northern analysis of $b Z I P 1$ specific transcripts using wt, T-DNA insertion lines (bzip1-1, bzip1bzip53) and plants overexpressing bZIP1 under control of the 35S promoter (Pro35S:HA-bZIP1). Ethidium Bromide staining (EtBr) has been used as a loading control. (D) Immunoblot analysis of protein extracts derived from the Pro35S:HA-bZIP1 lines indicated using a HA-tag 

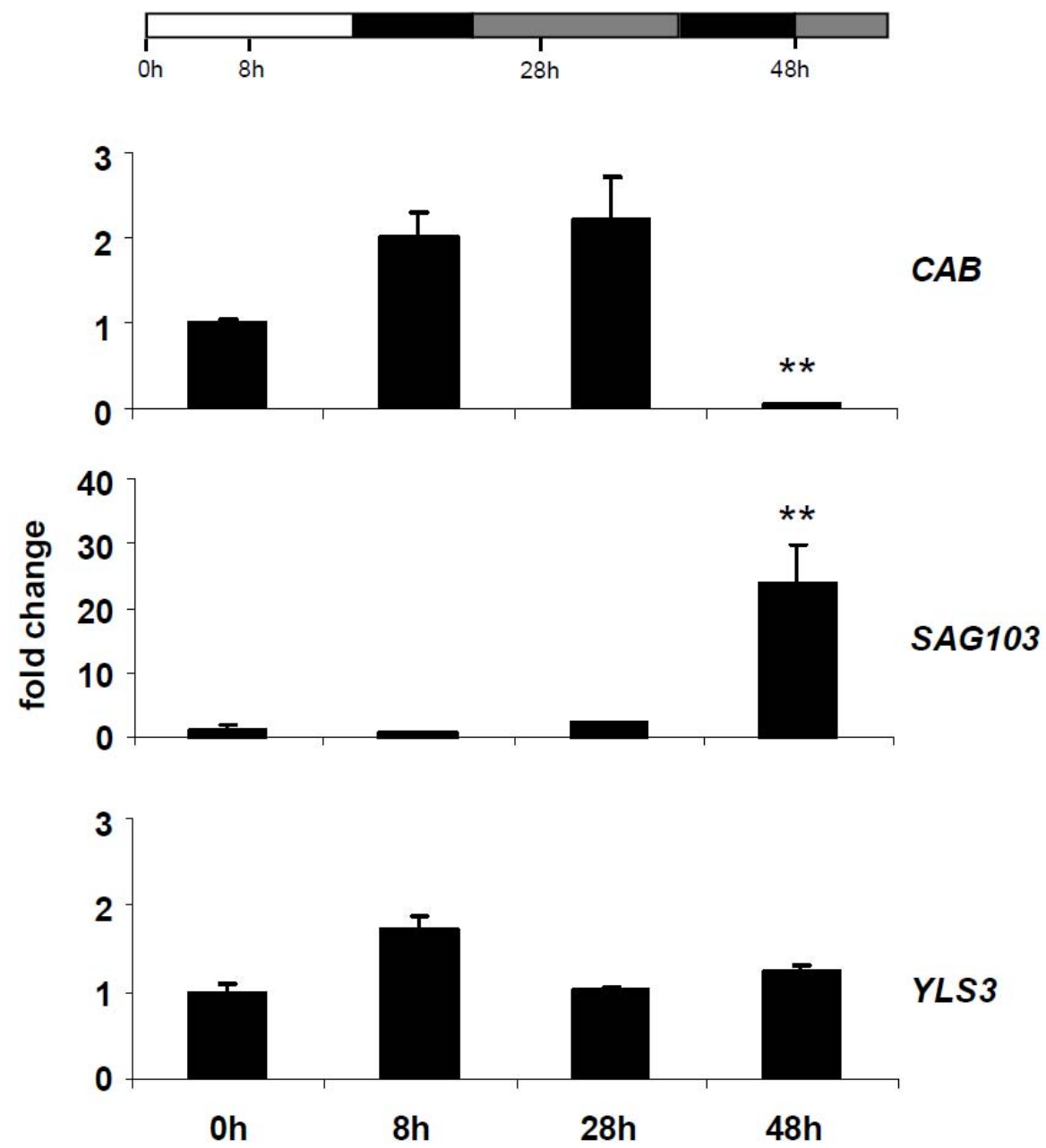

Supplemental Figure S3. Expression of senescence marker genes has been analysed in plants which have been cultivated under extended night treatment. Day, night and extended night phases are indicated by white, black or grey bars, respectively. RNA isolated at the time points indicated has been studied by qPCR. Whereas transcription of photosynthetic markers such as $C A B$ is strongly reduced within $48 \mathrm{~h}, S A G 103$ as a marker for dark-induced senescence related to energy deprivation (van der GRAAFF et al., 2006) is strongly induced in wt plants. This process is distinct from natural senescence as the expression of the correspondent marker gene (YLS3) is not changed. Asterisks (**) indicate values that were determined by one-way ANOVA and following Tukey's post-test to be significantly different $(p<0.01)$ in comparison to the other time points. 
A

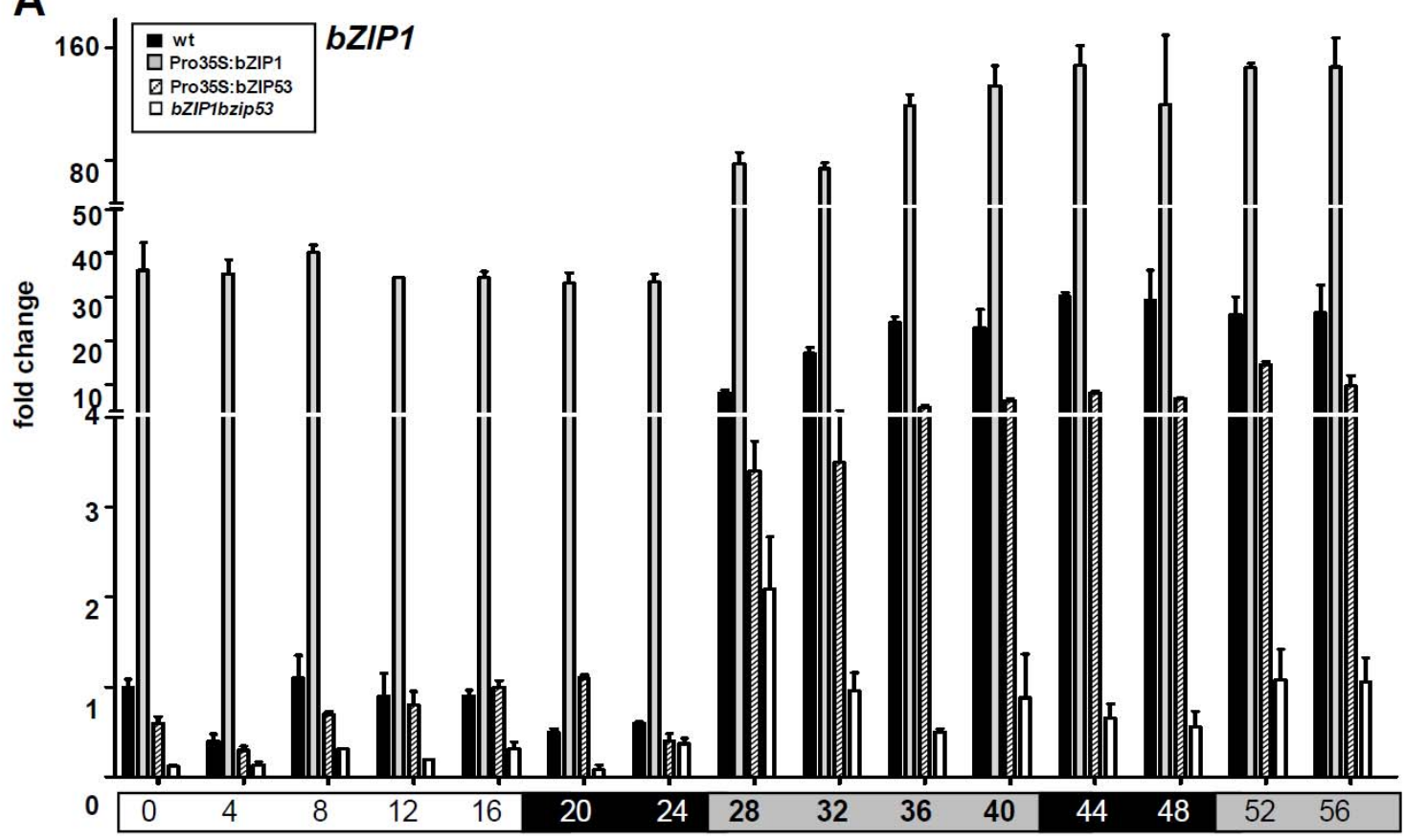

B

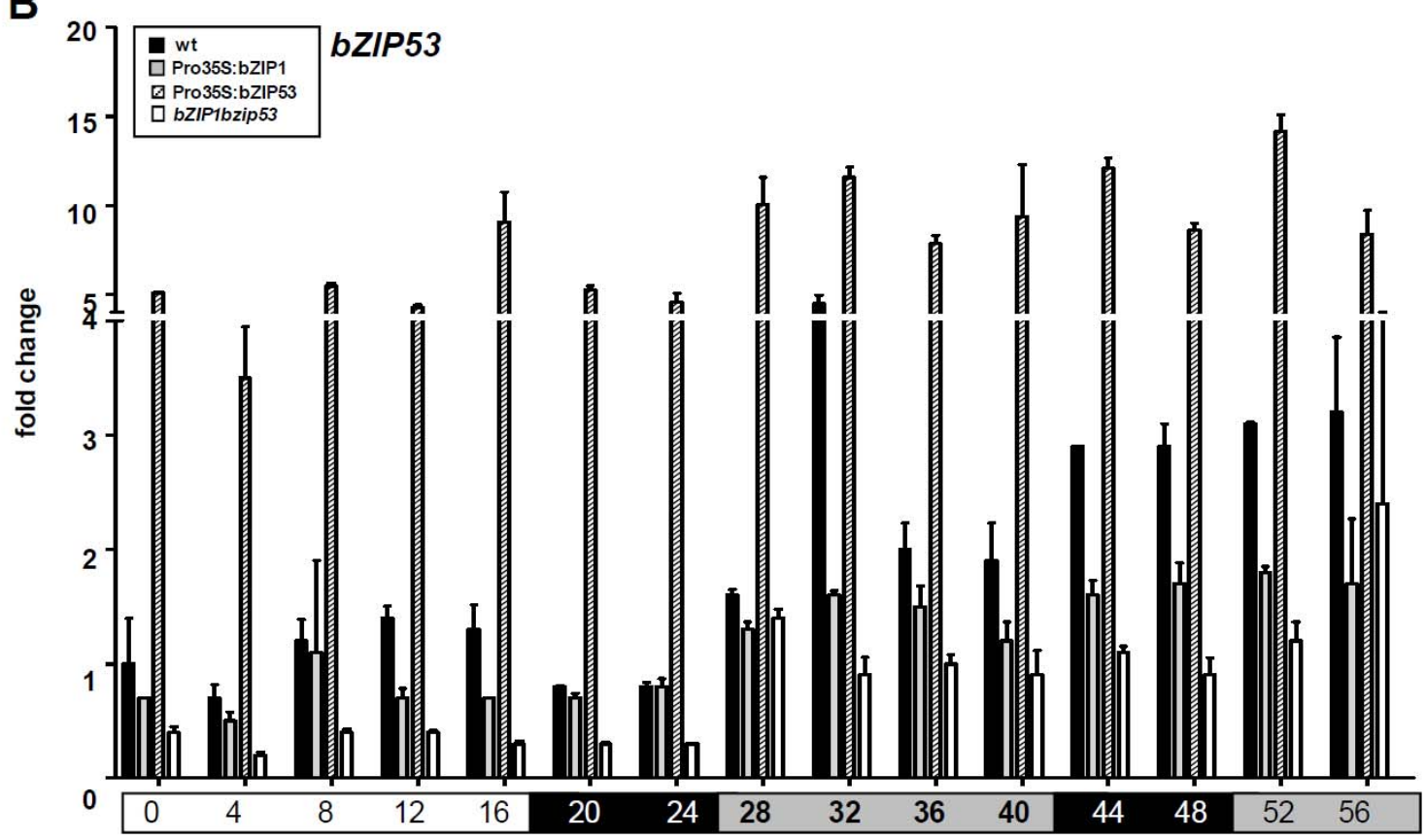

Supplemental Figure S4. Expression of bZIP1 and bZIP53 after extended night treatment as described in Figure 1A. Wt (black bars), overexpression plants under control of the $35 \mathrm{~S}$ promoter (Pro35S:bZIP1, grey bars; Pro35S:bZIP53, hatched bars) and a double T-DNA insertion line (bzip1bzip53, white bars) have been compared by qPCR with respect to bZIP1 (A) and bZIP53 (B) transcript abundance. Due to promoter insertion bzip53 is considered as a knock-down (WELTMEIER et al., 2006), whereas no significant amounts of bzip1 transcript can be observed. Error bars represent mean values of 2 repetitions. For visualizing the differences in transcript levels, the y axes is broken. 
A
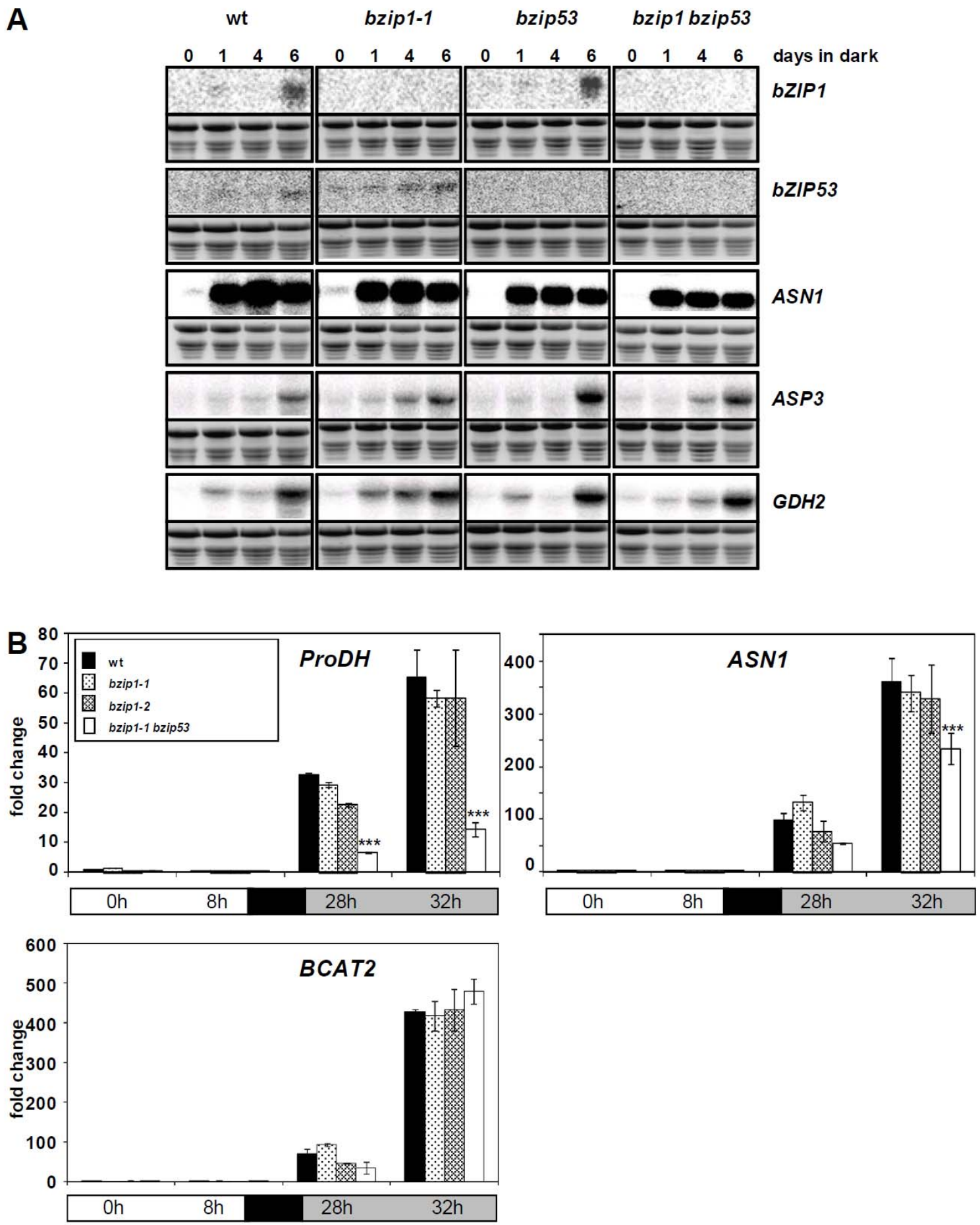

Supplemental Figure S5. Expression analysis of genes involved in amino acid metabolism analysed in single and double T-DNA insertion mutants of bZIP1 and bZIP53

(A) Northern analysis of the indicated genes after long-term dark treatment for $0-6$ days. Compared are wt, bzip1-1, bzip53 and bzip1 bzip53 plants. As a loading control, Ethidium bromide (EtBr) stainings are provided for each hybridisation experiment. (B) qPCR expression analysis of ASN1, ProDH and BCAT2 in the two T-DNA mutant lines bzip1-1, bzip1-2 and the double mutant bzip1 bzip53. The light/dark regime is given in the schematic drawing. Depicted is the fold change compared to wt at $0 \mathrm{~h}$. Asterisks represent significant differences of mutant plants compared to the wt at the indicated time point (twoway ANOVA, ${ }^{*} \mathrm{p}<0.05 ; * * \mathrm{p}<0.01 ; * * * \mathrm{p}<0.001$ ). 
A

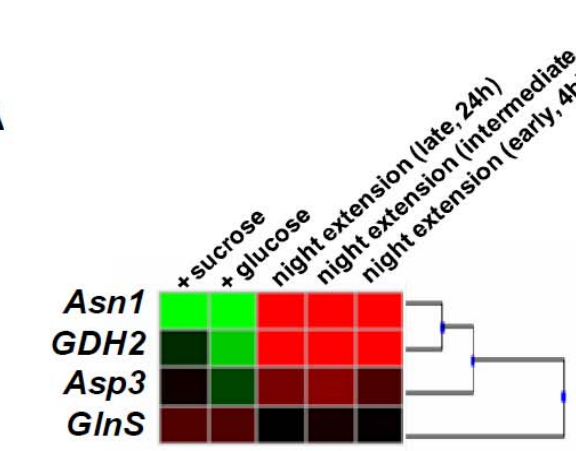

BCAT4

BCAT3

BCAT6

BCAT5

BCAT1

BCAT2

C
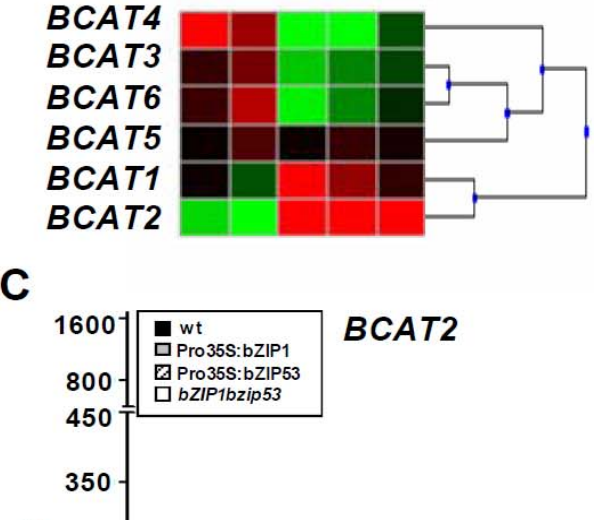

B

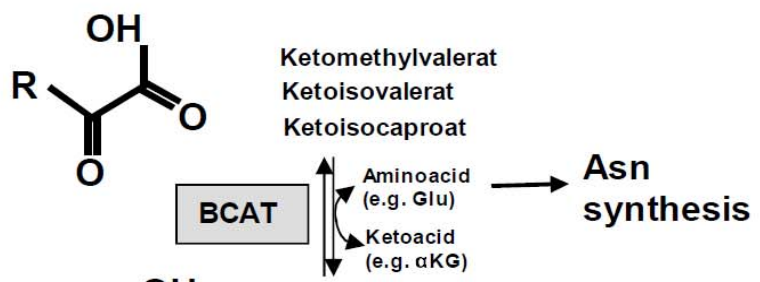

응

$\begin{array}{ll}\stackrel{0}{\mathrm{~T}} & 250 \\ \text { 단 } & \\ \text { 은 } & 150\end{array}$
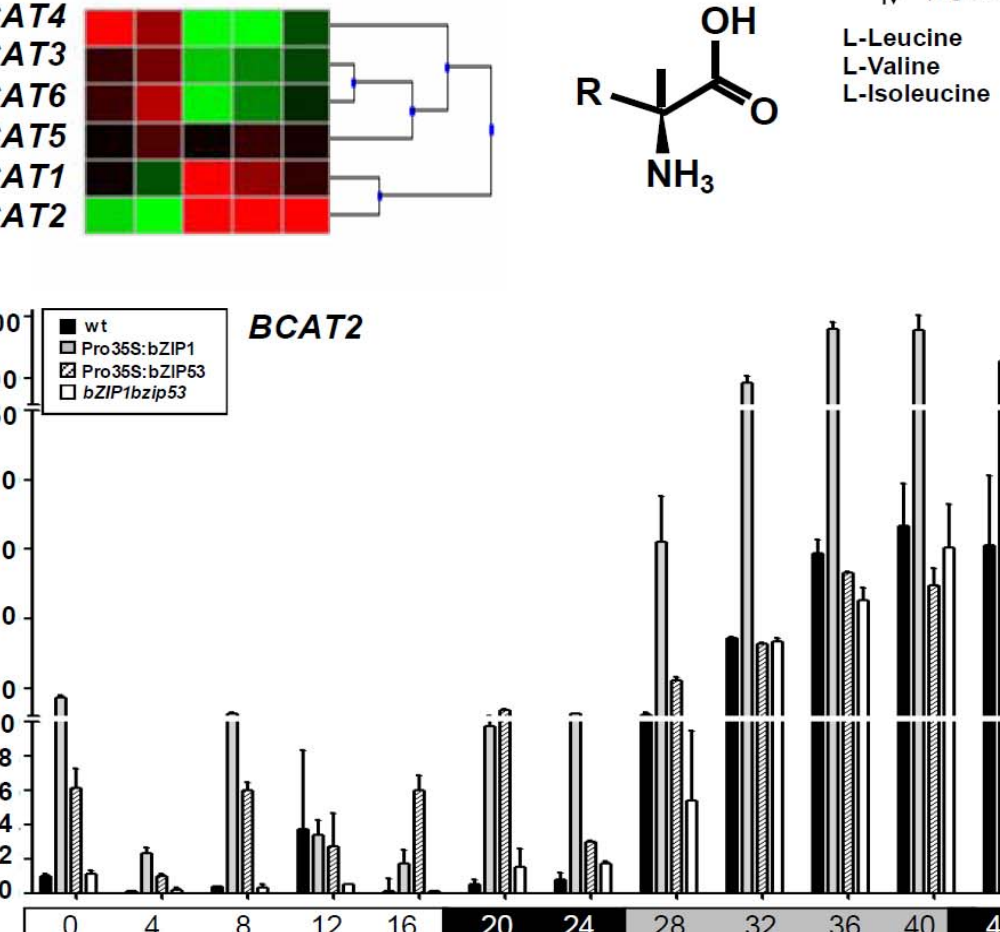

D

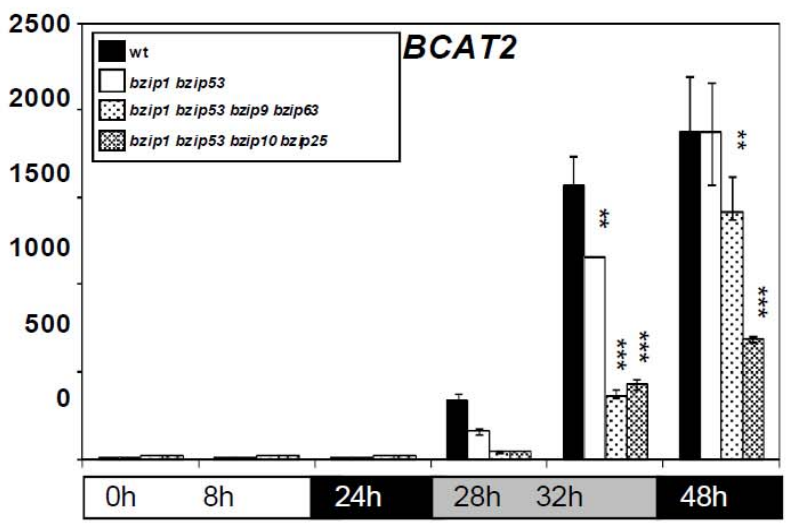


Supplemental Figure S6. Regulation of transcription in Branched Chain Amino Acid (BCAA) metabolism during dark-treatment

(A) Cluster analysis of the Asn biosynthesis and BCAT genes indicated making use of public expression data and the Genevestigator tool (Hruz et al., 2008). Transcriptome data sets analysing energy deprivation are compared at early, intermediate and late night extension ( $\mathrm{h}$ as indicated). Opposed are treatments with glucose or sucrose (for $4 \mathrm{~h}$ ), respectively. Red and green colours indicate induction or repression of the corresponding genes. (B) Schematic overview of the BCAA metabolism. $\alpha \mathrm{KG}, \alpha$-keto-glutarate. (C) Induction of BCAT2 after shortterm dark treatment. Wt (black bars), Pro35S:bZIP1 (grey bars), Pro35S:bZIP53 (hatched bars) and the corresponding T-DNA insertion line (bzip1 bzip53, white bars) have been cultivated at a day/night rhythm of $16 / 8 \mathrm{~h}$ as indicated by the scheme at the bottom. Day, night and extended night phases are indicated by white, black or grey bars, respectively. Rosette leaves of 10 three week-old plants have been pooled and used for RNA preparation and qPCR at the time points indicated. In comparison to the transcript level of wt plants at "0" time point, BCAT2 transcript abundance of the time points indicated is depicted as fold change. For visualizing the differences in transcript levels, the y axes is broken at 10 and 450 fold induction. (D) qPCR analysis of BCAT2 transcript accumulation after extended night treatment in several bZIP multiple mutant lines as described in Figure 6C. Asterisks represent significant differences of mutant plants compared to the wt at the indicated time point (two-way ANOVA, * p < $0.05 ; * * \mathrm{p}<0.01 ; * * * \mathrm{p}<0.001)$. 
A

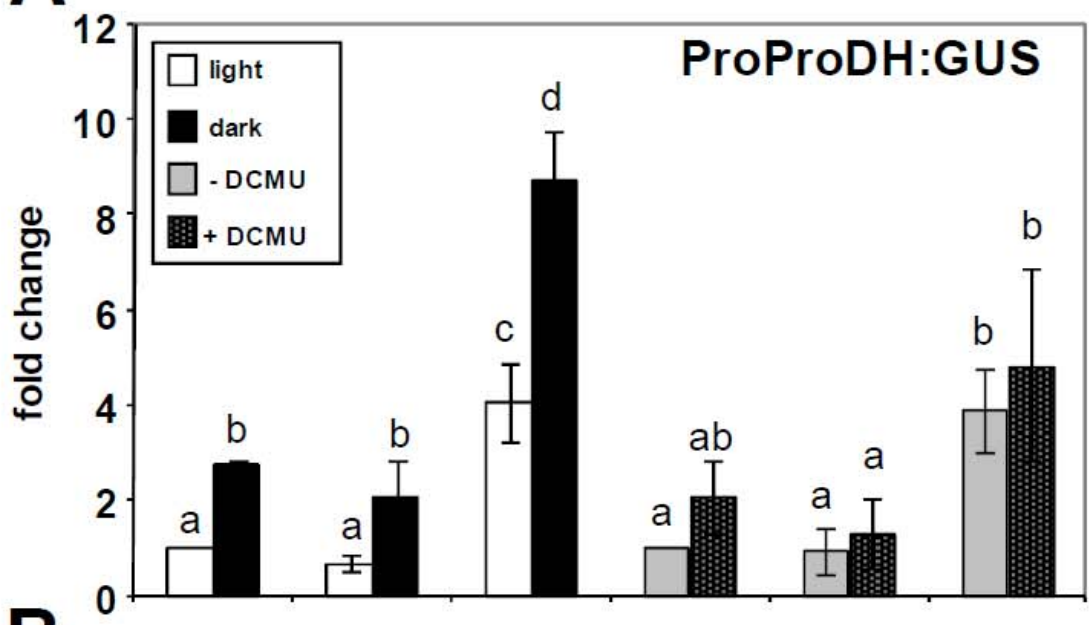

B

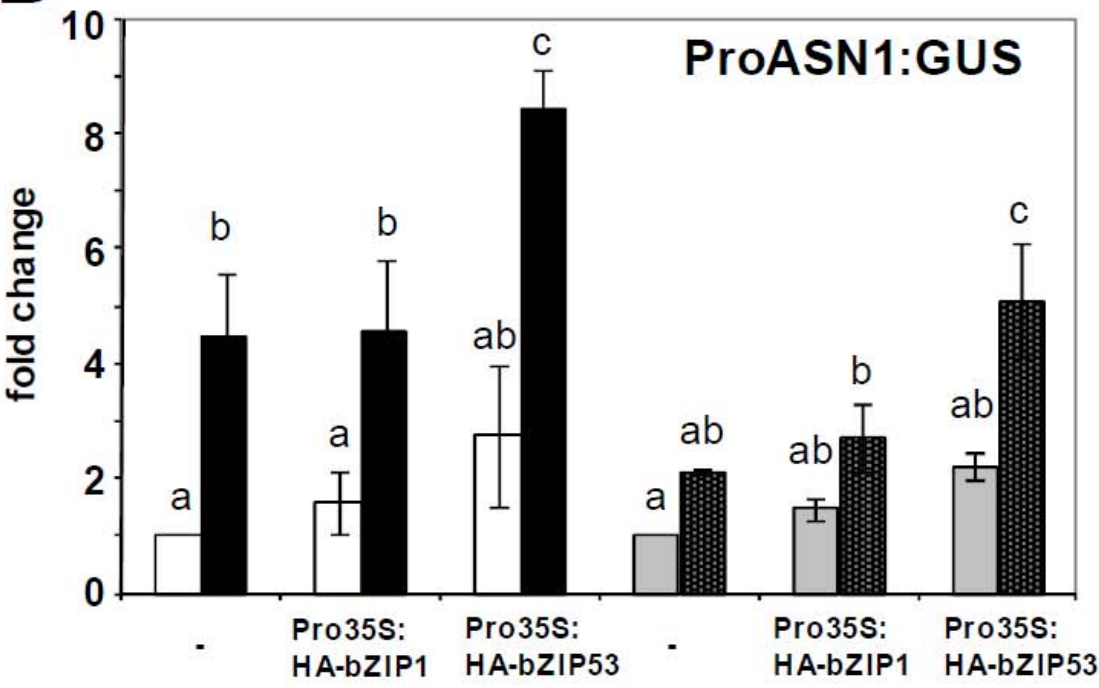

C
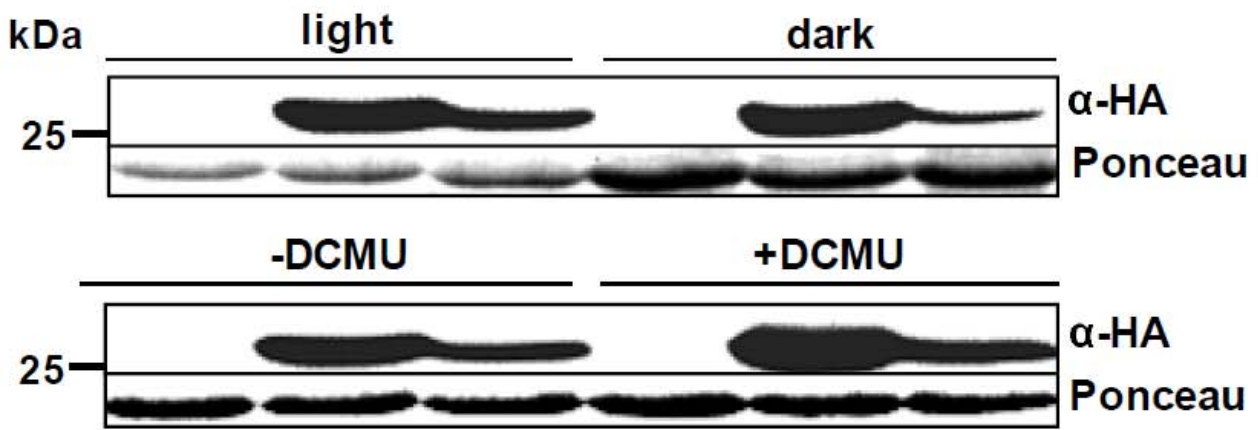

- Pro35S: Pro35S: - Pro35S: Pro35S:

HA-bZIP1 HA-bZIP53 - HA-bZIP1 HA-bZIP53 
Supplemental Figure S7. Analysis of energy deprivation induced transcription in protoplasts Promoter:GUS reporter constructs of the bZIP target genes, namely ProDH (A) and ASN1 (B) have been used in transiently transformed protoplast assays. Induction of the reporters has been observed after constant dark treatment (black bars) in comparison to constant light treatment (white bars) for 16 h. Comparable induction has been observed by treatment with the photosystem II inhibitor DCMU (dark grey bars) in comparison to untreated cells (light grey bars). GUS activity has been determined after co-transformation with $14 \mu \mathrm{g}$ of Pro35S:HAProDH bZIP1 or Pro35S:HA-bZIP53 effector plasmids or an empty vector control (-). Inclusion of $1 \mu \mathrm{g}$ of a reference plasmid encoding a Pro35S:NAN gene has been used in all experiments to normalize GUS expression values for differences in transfection efficiencies as described by (Ehlert et al., 2006). Y-axis values are expressed as fold change in comparison to the control incubation under constant light. Given are mean values of 4 independent transfections. Significance of light/dark and +/- DCMU treatment was tested by one-way ANOVA analysis following Tukey's post-test, $\mathrm{p}<0.05$, respectively. (C) Expression of the HAtagged bZIP effectors has been studied by immunoblot analysis in light/dark grown or DCMU treated/untreated protoplasts using a HA-tag specific antibody $(\alpha \mathrm{HA})$. For loading control Ponceau staining has been used. Protein size is compared to size standard in kilo Dalton $(\mathrm{kDa})$.

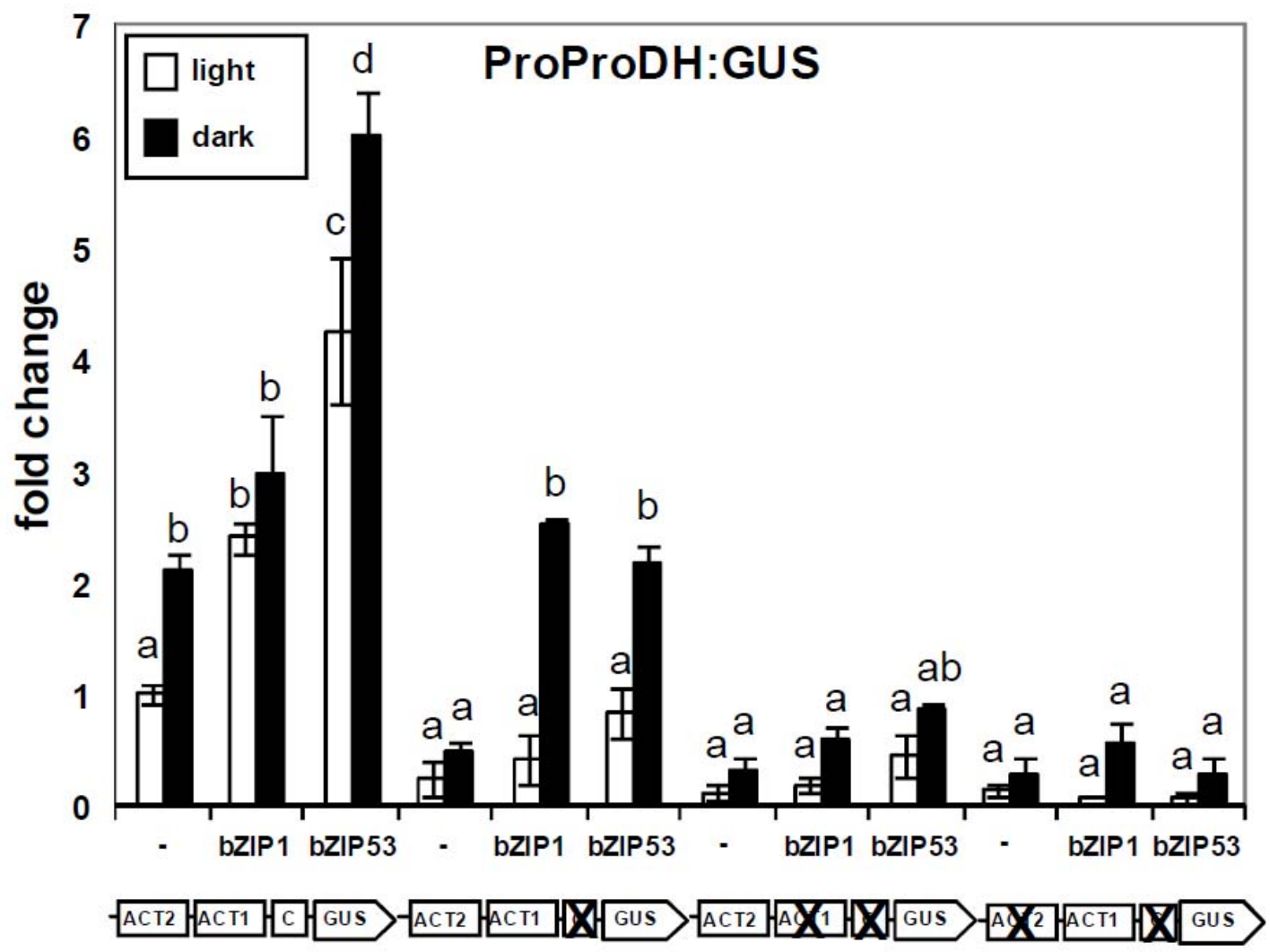

\section{Supplemental Figure S8. Mutation in the C-box effects ProDH promoter}

activity. Transient transformation of protoplasts has been performed as described in Figure 5 with the promoter mutations indicated. Depicted is the fold change compared to the unmutated promoter in the light. Significance was tested by One-way ANOVA analysis following Tukey's post-test, $\mathrm{p}<0.05$. 
A

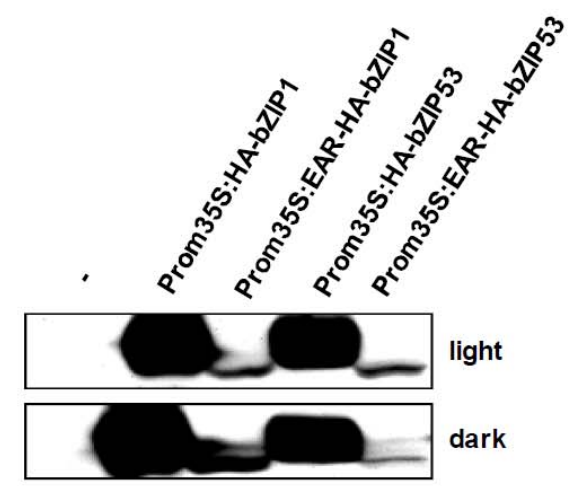

B

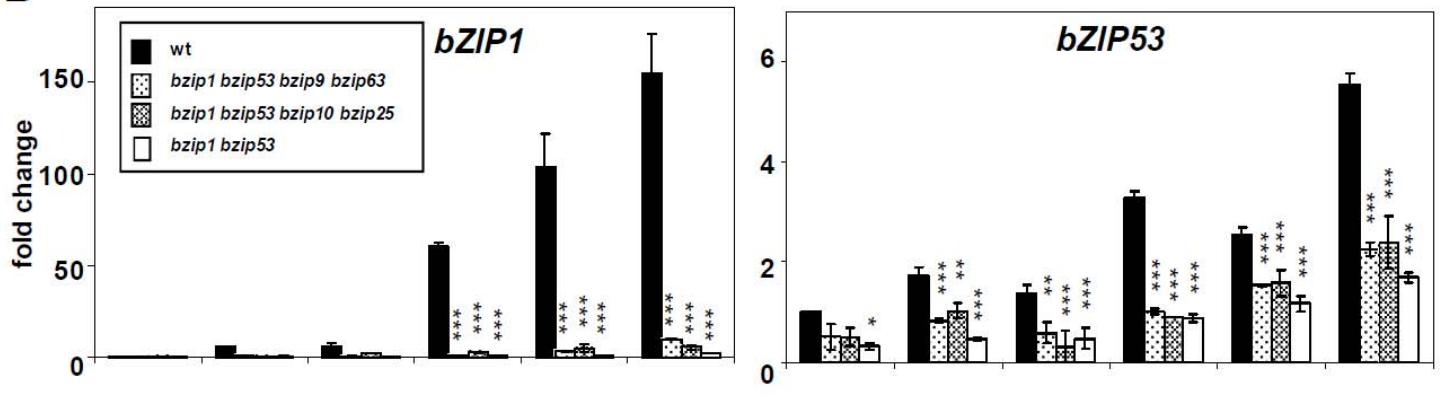

C

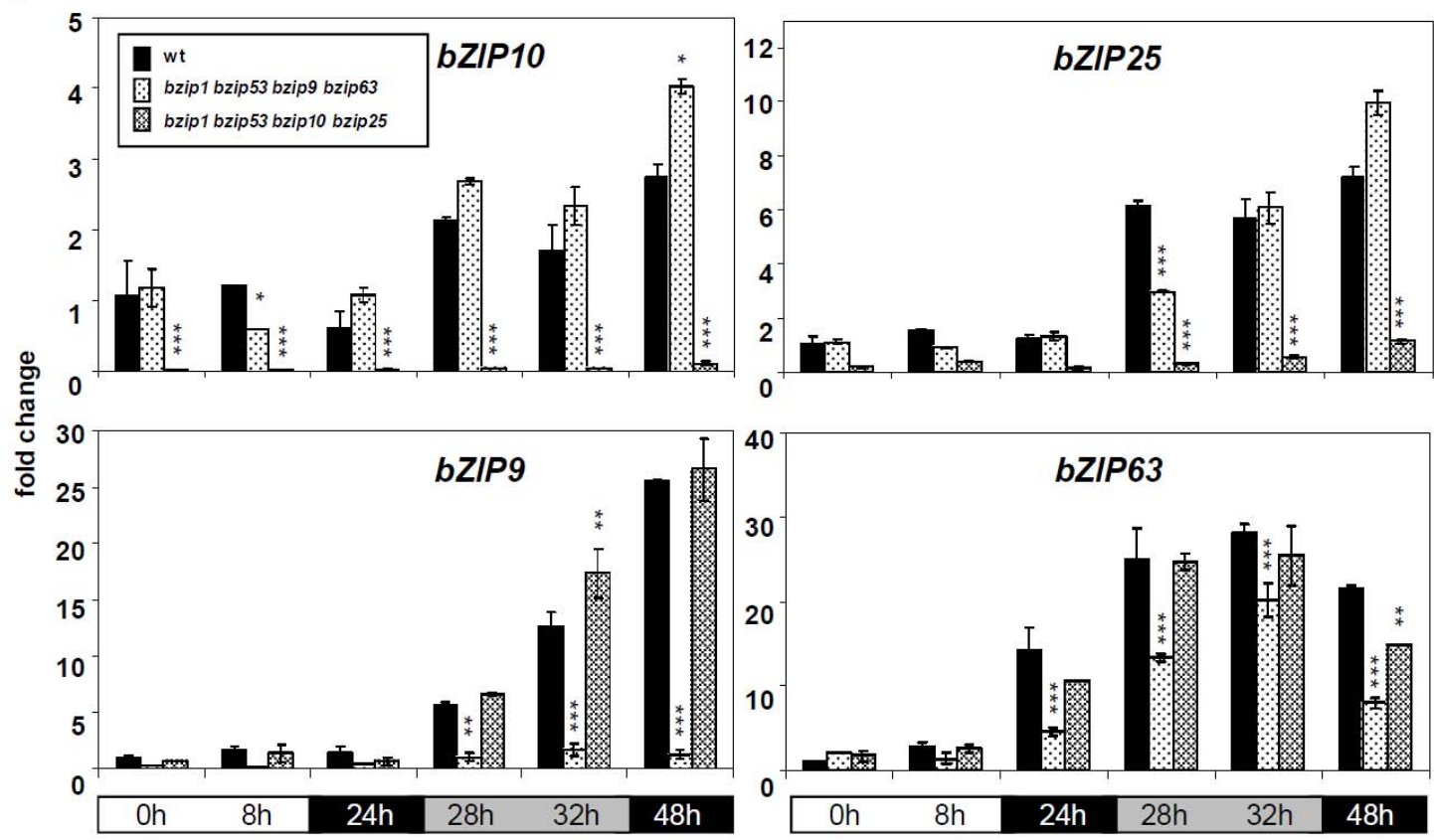


Supplemental Figure S9. Immunoblot analysis of the EAR repressor approach and characterisation of multiple T-DNA insertion mutants used in this study

(A) The Expression of HA-bZIP1, HA-bZIP53, EAR-HA-bZIP1 and EAR-HAbZIP53 in Figure 6 A in constant light (upper panel) or darkness (lower panel) was monitored by immunoblot analysis using an $\alpha$-HA antibody. Since the EAR domain fusions harbour only two HA epitopes in comparison to the constructs without EAR domain (3 epitopes), the expression levels can only be compared between the related protein fusions. (B) Expression analysis of bZIP1 and bZIP53 performed by qPCR using the indicated T-DNA insertion lines. (C) Expression analysis of group C bZIP10, bZIP25, bZIP9, bZIP63 using the indicated T-DNA insertion lines. Depicted is the fold change compared to wt at $0 \mathrm{~h}$. Asterisks represent significant differences between mutant plants and the wt at the indicated time point (two-way ANOVA, $* \mathrm{p}<0.05 ; * * \mathrm{p}<0.01 ; * * * \mathrm{p}<0.001$ ). 


\begin{tabular}{|c|c|c|c|}
\hline Gene & AGI code & $\begin{array}{l}\text { ACGT-like elements } \\
\text { (-1000 bp promoter region) }\end{array}$ & Box \\
\hline ProDH & At3g30775 & $\begin{array}{l}-842 \text { CACTCATcct }-833 \\
-714 \text { gACTCATcct }-705 \\
-683 \text { ctgACGTcct }-674 \\
-572 \text { ataACGTagc }-563 \\
-197 \text { aaaACGTgta }-188 \\
-171 \text { ttACGTgct }-162\end{array}$ & $\begin{array}{l}\text { ACT2 } \\
\text { ACT1 } \\
\text { C } \\
\text { T/A } \\
\text { T/G } \\
\text { A/G }\end{array}$ \\
\hline ASN1 & At3g47340 & $\begin{array}{l}-731 \text { ttaACGTttt }-722 \\
-557 \text { aacACGTgta }-548 \\
-377 \text { cggACGTcgt }-368 \\
-354 \text { aacACGTgga }-345 \\
-167 \text { tctACGTgca }-158\end{array}$ & $\begin{array}{l}\text { T } \\
\text { G2 } \\
\text { C } \\
\text { G1 } \\
\text { A/G }\end{array}$ \\
\hline GDH2 & At5g07440 & $\begin{array}{l}-974 \text { ctaACGTgga }-965 \\
-737 \text { tccACGTccc }-728 \\
-282 \text { ctgACGTagg }-273 \\
-273 \text { gatACGTcaa }-264\end{array}$ & $\begin{array}{l}T / G \\
G / C \\
C / A \\
A / C\end{array}$ \\
\hline$A S P 3$ & At5g11520 & $\begin{array}{l}-841 \text { tcaACGTggt }-832 \\
-480 \text { tatACGTtga }-471 \\
-122 \text { cttACGTggc }-113\end{array}$ & $\begin{array}{l}\text { T/G } \\
A / T \\
A / G\end{array}$ \\
\hline GLNS & At5g37600 & $-157 \mathrm{ttcACGTCac}-148$ & G/C \\
\hline PepCK & At5g65690 & $\begin{array}{l}-738 \text { aaaACGTgct }-729 \\
-524 \text { caaACGT gaa }-515 \\
-467 \text { tgcACGTaaa }-458 \\
-423 \text { tcaACGTaat }-414 \\
-334 \text { ataACGTgag }-325 \\
-76 \text { tatACGTgta }-67\end{array}$ & $\begin{array}{l}\text { T/G } \\
\text { T/G } \\
\text { G/A } \\
\text { T/A } \\
\text { T/G } \\
\text { A/G }\end{array}$ \\
\hline ANS & At3g16150 & $\begin{array}{l}-882 \text { attACGTaag }-873 \\
-776 \text { ttgACGTata }-767 \\
-752 \text { ataACGTaga }-743 \\
-511 \text { ctgACGTgga }-502 \\
-361 \text { ataACGTttc }-352\end{array}$ & $\begin{array}{l}\text { A } \\
\text { C/A } \\
\text { T/A } \\
\text { C/G } \\
\text { T }\end{array}$ \\
\hline BCAT2 & At1g10070 & $\begin{array}{l}-804 \text { aaAACGTtgt }-795 \\
-770 \text { ctgACGTggc }-761 \\
-749 \text { acaACGTgtt }-740 \\
-712 \text { aagACGTtga }-703 \\
-633 \text { ccaACGTggc }-624 \\
-337 \text { gacACGTcta }-328 \\
-164 \text { cttACGTgtt }-155\end{array}$ & $\begin{array}{l}T \\
C / G \\
T / G \\
C / T \\
T / G \\
\text { G/C } \\
\text { A/G }\end{array}$ \\
\hline
\end{tabular}

Supplemental Table 2 Summary of putative bZIP binding motifs (ACGT- like elements) found in the promoters (1 kb upstream region) of genes related to Asn, Pro and BCAT metabolism. Genes, AGI code and the putative elements are given. 


\begin{tabular}{|c|c|c|c|}
\hline Mutant & SALK Nr. & Insertion in & $\begin{array}{l}\text { Knock down / Knock } \\
\text { out }\end{array}$ \\
\hline bzip1 & SALK_059343 & ORF & Knock out \\
\hline bzip53 & SALK_069883 & 5'UTR & Knock down \\
\hline bzip1 bzip53 & $\begin{array}{l}\text { SALK_059343 } \\
\text { SALK_069883 }\end{array}$ & $\begin{array}{l}\text { ORF } \\
\text { 5'UTR }\end{array}$ & $\begin{array}{l}\text { Knock out } \\
\text { Knock down }\end{array}$ \\
\hline bzip1 bzip53 bzip9 bzip63 & $\begin{array}{l}\text { SALK_059343 } \\
\text { SALK_069883 } \\
\text { SALK_093416 } \\
\text { SALK_066531 }\end{array}$ & $\begin{array}{l}\text { ORF } \\
\text { 5'UTR } \\
\text { 3. Exon } \\
\text { 5'UTR }\end{array}$ & $\begin{array}{l}\text { Knock out } \\
\text { Knock down } \\
\text { Knock out } \\
\text { Knock down }\end{array}$ \\
\hline bzip1 bzip53 bzip10 bzip25 & $\begin{array}{l}\text { SALK_059343 } \\
\text { SALK_069883 } \\
\text { SALK_106031 } \\
\text { SALK_119931 }\end{array}$ & $\begin{array}{l}\text { ORF } \\
\text { 5'UTR } \\
\text { 3. Exon } \\
\text { 1. Exon }\end{array}$ & $\begin{array}{l}\text { Knock out } \\
\text { Knock down } \\
\text { Knock out } \\
\text { Knock out }\end{array}$ \\
\hline
\end{tabular}

Supplemental Table 3 Summary of the T-DNA insertion lines used in this study.

qPCR Primer:

\begin{tabular}{|c|c|c|}
\hline SAG103 & At1g10140 & $\begin{array}{l}\text { 5'-AGCTCGAGTGCTGGGATG-3' } \\
\text { 5'-CGGATTCACAGATCCTTCCT-3' }\end{array}$ \\
\hline CAB & At1g29920 & $\begin{array}{l}\text { 5'-TCAATCTTTTGAATTCGAGTGAGA-3' } \\
\text { 5'-TCCACCACAAACACAAACCTAC-3' }\end{array}$ \\
\hline YLS3 & At2g44290 & $\begin{array}{l}\text { 5'-GACATCACTAAGTGCCCTGCT-3' } \\
\text { 5'-ACTGTTTCGTTCAGACCTTTAGC-3' }\end{array}$ \\
\hline ASN1 & At3g47340 & $\begin{array}{l}\text { 5'-TTCTTGAGCTTTCTCGCAGAT-3' } \\
\text { 5'-CCGTTCTGATATAAGCCACTCC-3' }\end{array}$ \\
\hline ProDH & At3g30775 & $\begin{array}{l}\text { 5'-CGCCAGTCCACGACACAATTCA-3' } \\
\text { 5'-CGAATCAGCGTTATGTGTTGCG-3' }\end{array}$ \\
\hline ANS & At3g16150 & $\begin{array}{l}\text { 5'-TTCCGATCAGCATTTACGC-3', } \\
\text { 5'-CAATGTCCTTTCCCGTCAAC-3' }\end{array}$ \\
\hline BCAT2 & At1g10070 & QuantiTect@ Primer QT00854875 \\
\hline bZIP1 & At5g49450 & $\begin{array}{l}\text { 5'-TCAGCGTTAAACTCGTCGTAGCAA-3', } \\
\text { 5'-AACGCGGGTCTTAGATCGGAGAAG-3' }\end{array}$ \\
\hline bZIP53 & At3g62420 & $\begin{array}{l}\text { 5'-TGGGGTCGTTGCAAATGCAAACAA-3' } \\
\text { 5'-CCGTGGCGTACCTCGGATCATTAT-3' }\end{array}$ \\
\hline bZIP9 & At5g24800 & $\begin{array}{l}\text { 5'-CGAAAAGGTCCAGCCGGAAACAAT-3' } \\
\text { 5'-TCGACCTCATGAACCGGGATTACA-3' }\end{array}$ \\
\hline bZIP10 & At4g02640 & $\begin{array}{l}\text { 5'-TTTTTCGGCCATGCTGAATCGTTC-3' } \\
\text { 5'-TTACTCCAAGCGCCAACCCGTA-3' }\end{array}$ \\
\hline bZIP25 & At3g54620 & $\begin{array}{l}\text { 5'-AGGAGGATGCTCTCAAACCGAGAA-3' } \\
\text { 5'-CGGCTCTTAATTGGCCTACCTGTG-3' }\end{array}$ \\
\hline bZIP63 & At5g28770 & $\begin{array}{l}\text { 5'-AAAAGGGGAACTTTCATCAAACCTCAGG. } \\
\text { 3' } \\
\text { 5'-CTGAGGCATATTGTGGAACATTGGGT-3' }\end{array}$ \\
\hline UBQ5 & At3g62250 & $\begin{array}{l}\text { 5'-GACGCTTCATCTCGTCC-3' } \\
\text { 5'-GTAAACGTAGGTGAGTCCA-3' }\end{array}$ \\
\hline
\end{tabular}

ChIP Primer:

\begin{tabular}{|l|l|l|}
\hline ASN1 & At3g47340 & $\begin{array}{l}\text { 5'-CACGTGTACGGCTCTAAAGCA-3' } \\
\text { 5'-GACCAGCTGTTTCCACGTGTT-3' }\end{array}$ \\
\hline ProDH & At3g30775 & $\begin{array}{l}\text { 5'-CAAGTCCAGGTCCACATGTTG-3' } \\
\text { 5'-CAAGGCCCTGACCATAGGA-3' }\end{array}$ \\
\hline ACT7 & At5g09810 & $\begin{array}{l}\text { 5'-CGTTTCGCTTTCCTTAGTGTTAGCT-3' } \\
\text { 5'-AGCGAACGGATCTAGAGACTCACCTTG-3' }\end{array}$ \\
\hline
\end{tabular}

Supplemental Table 4 Summary of the oligonucleotide primers used in this study. 
Primer to generate Northern probes:

\begin{tabular}{|l|l|l|}
\hline Asn1 & At3g47340 & $\begin{array}{l}\text { 5'-GTGGAATACTTGCCGTGTTAG-3' } \\
\text { 5'-CGAAATGCTCACAGTCATCG-3' }\end{array}$ \\
\hline GDH2 & At5g07440 & $\begin{array}{l}\text { 5'-GCTGCAACAAACAGAAACTTCC-3' } \\
\text { 5'-CCCTGAATCGATTTCCCGT-3' }\end{array}$ \\
\hline ProDH & At3g30775 & $\begin{array}{l}\text { 5'-ATGGCAACCCGTCTTCTCCG-3' } \\
\text { 5'-TGGTCGGTCTTTGTCAGCAT-3' }\end{array}$ \\
\hline BCAT2 & At1g10070 & $\begin{array}{l}\text { 5'-CACATCTCTACGCAAAACTCTG-3' } \\
\text { 5'-TGTGATGCTCTTGACGCCTC-3' }\end{array}$ \\
\hline bZIP1 & At5g49450 & $\begin{array}{l}\text { 5'-GGCAAACGCAGAGAAGACAA-3' } \\
\text { 5'-GGACGCCATTGGTTGTAGAG-3' }\end{array}$ \\
\hline bZIP53 & At3g62420 & $\begin{array}{l}\text { 5'-GTGCTTGAGATGGTTGAAGAAATTA-3' } \\
\text { 5'-CATACAAAGACACGAACAAATTGAA-3' }\end{array}$ \\
\hline PepCK & At5g65690 & $\begin{array}{l}\text { 5'-ATGGCGGGAAACGGAAACGA-3' } \\
\text { 5'-TCGTACAACTCGGCGGGAGA-3' }\end{array}$ \\
\hline Asp3 & At5g11520 & $\begin{array}{l}\text { 5'-CTTCCTCTTCTTCTTCCGATCG-3' } \\
\text { 5'-GTCGCTGGATCGTAGTATCGG-3' }\end{array}$ \\
\hline
\end{tabular}

Primer for test of T-DNA insertion:

\begin{tabular}{|l|l|l|}
\hline bZIP1 & At5g49450 & $\begin{array}{l}\text { 5'-ATGGCAAACGCAGAGAAGACAAGTTCA- } \\
\text { 3' } \\
\text { 5'-TCATGTCTTAAAGGACGCCATTGGTTG-3' }\end{array}$ \\
\hline bZIP53 & At3g62420 & $\begin{array}{l}\text { 5'-CTGCAGCTTAGGACAGCTCATCACCA-3' } \\
\text { 5'-GTCGACCTCGTTGACTTTTTGACTTC-3' }\end{array}$ \\
\hline bZIP9 & At5g24800 & $\begin{array}{l}\text { 5'-ATGGATAATCACACAGCTAAAGA -3' } \\
\text { 5'-GAGTTATCGCCTTTCAAAGA-3' }\end{array}$ \\
\hline bZIP10 & At4g02640 & $\begin{array}{l}\text { 5'-TTGAGATGAGTTCTGGTGGTTA-3' } \\
\text { 5'-GTCCACTTGATCTTCCGAGAA-3' }\end{array}$ \\
\hline bZIP25 & At3g54620 & $\begin{array}{l}\text { 5'-ATGCACATCGTCTTCTCTGTCG-3' } \\
\text { 5'-TGCCTTGTTTCTTTCTTCTCAG-3' }\end{array}$ \\
\hline bZIP63 & At5g28770 & 5'-CGTAGCTCGGTCATTGTGTCGG-3' \\
& 5'-TCTCCGTCGTCTGCAGCGGC-3' \\
\hline LBa1 & & 5'-'TGGTTCACGTAGTGGGCCATCG -3' \\
\hline
\end{tabular}

Supplemental Table 4 cont. 
Primer for construction of promotor-GUS constructs :

\begin{tabular}{|c|c|c|}
\hline ASN1 promoter & $\begin{array}{l}\text { with Xbal und Ncol } \\
\text { restriction sites }\end{array}$ & $\begin{array}{l}\text { 5'-AAAAAAAAAATCTAGACGTCTCAAGTATTGATAAAATA } \\
\text { ATAAT-3' } \\
\text { 5'-AAAAAAAAAACCATGGGTTTTTTTTTTTGAAGAAAGTGA } \\
\text { AAAAGATCACGAAGAA-3' }\end{array}$ \\
\hline ProDH promoter & $\begin{array}{l}\text { with Pstl and Ncol } \\
\text { restriction sites }\end{array}$ & $\begin{array}{l}\text { 5'- } \\
\text { AAAAAAAAAACTGCAGACTTGCACTAAAGACTAAACAGT-3' } \\
\text { 5'-AAAAAAAAAACCATGGAAAATTCAAAGATTTTGTTTTTGA } \\
\text { AAACGAAAACAAA-3' }\end{array}$ \\
\hline $\begin{array}{l}\text { ProDH promoter } \\
\text { ACTCAT1 mut }\end{array}$ & ACTCAT1 mutation & $\begin{array}{l}\text { 5'-TATATTAATTAAATAGTTATCCGATGTTACATCCTATGG } \\
\text { TCAGGGCCTTGCTGTC-3' } \\
\text { 5'-GACAGCAAGGCCCTGACCATAGGATGTAACATCGGAT } \\
\text { AACTATTTAATTAATATA-3' }\end{array}$ \\
\hline $\begin{array}{l}\text { ProDH promoter } \\
\text { ACTCAT2 mut }\end{array}$ & ACTCAT2 mutation & $\begin{array}{l}\text { 5'-ATGTTGAATCCGATTCATCATCCTTACATCCTTCATATC } \\
\text { TTCCTCCACC-3' } \\
\text { 5'-GGTGGAGGAAGATATGAAGGATGTAAGGATGATGAAT } \\
\text { CGGATTCAACAT-3' }\end{array}$ \\
\hline $\begin{array}{l}\text { ProDH promoter } \\
\text { C-box mut }\end{array}$ & C-box mutation & $\begin{array}{l}\text { 5'-CTATGGTCAGGGCCTTGCTGTCTCTGAAATCCTTAATT } \\
\text { ATCATTAT-3' } \\
\text { 5'-ATAATGATAATTAAGGATTTCAGAGACAGCAAGGCCCT } \\
\text { GACCATAG-3' }\end{array}$ \\
\hline $\begin{array}{l}\text { ASN1 promoter } \\
\text { G1-box mut }\end{array}$ & G1 mutation & $\begin{array}{l}\text { 5'-CTAGTCTAGAGCAGGCTCATTTCGCGGAAAGTTG-3' } \\
\text { 5'-GTCTTCCATGGTGTTATCAACCAC-3' }\end{array}$ \\
\hline $\begin{array}{l}\text { Asn1 promoter } \\
\text { G2-box mut }\end{array}$ & G2 mutation & $\begin{array}{l}\text { 5'-CTAGTCTAGAGCAGGCTCATTTCGCGGAAAGTTG-3' } \\
\text { 5'-GTCTTCCATGGTGTTATCAACCAC-3' }\end{array}$ \\
\hline
\end{tabular}

Supplemental Table 4 cont.

\section{SUPPLEMENTAL METHODS}

\section{Molecular Biology and physiology Methods}

Northern analysis and histochemical GUS staining has been described in Weltmeier et al. (2006, 2009). Hybridisation probes were PCR generated using the primers summarized in Table S4. Northern analysis have been repeated three times with comparable results. Immunoblot analysis was performed according to Weltmeier et al. (2006) using $\alpha \mathrm{HA}$ antisera (SantaCruz, Ca, USA). Aa analysis was performed as described in Pilot et al (2004).

\section{Reverse transcription quantitative PCR}

RNA was isolated from pooled material of the rosettes of 103 -week old plants. cDNA synthesis and qPCR analysis was done as described in (Fode et al., 2009). Cycling conditions were as follows: 10 min at $95^{\circ} \mathrm{C}, 40$ cycles of $20 \mathrm{~s}$ at $95^{\circ} \mathrm{C}, 10 \mathrm{~s}$ at $55^{\circ} \mathrm{C}$ and $30 \mathrm{~s}$ at $72^{\circ} \mathrm{C}$, linked to a default dissociation stage program to detect non-specific amplification. The ubiquitin (UBI5) gene was used for sample normalization. Other gene specific oligonucleotides were described in Supplemental Table S4 online.

\section{Transient expression assays and constructs}

Protoplast isolation, transformation, construction of effector plasmids and Western analysis was performed according to Ehlert et al. (2006) and Weltmeier et al. (2006). ProProDH:GUS and ProASN1:GUS reporter constructs were obtained by inserting the PCR amplified promoters into the vector pBT10-TATA-GUS (Sprenger-Haussels and Weisshaar, 2000) using PstI/NcoI or XbaI/NcoI restriction sites, respectively. The PCR primers are given in Supplemental Table 4 online. Mutation of promoter cis elements has been performed using the Quick Change site-directed mutagenesis kit (Stratagene, Amsterdam, NL). For reporter gene assays, $5 \mu \mathrm{g}$ reporter and $14 \mu \mathrm{g}$ of effector were used if not stated otherwise. $1 \mu \mathrm{g}$ of Pro35S:NAN plasmid was added for normalization (Kirby and Kavanagh, 2002). Protoplasts were incubated over night for $16 \mathrm{~h}$ in constant light or constant darkness, respectively. For treatment with DCMU, $20 \mu \mathrm{M}$ of DCMU were added to the WI solution for incubation over night. Four transfection Dietrich et al., Function of bZIP factors in starvation response experiments were used to calculate mean values and standard errors/deviations of relative GUS/NAN activities as described in Ehlert et al. (2006). 


\section{Chromatin immunoprecipitation (ChIP)}

ChIP was performed as described in Weltmeier et al. (2006) and Alonso et al. (2009) using an HA-specific antibody (Abcam, Cambridge, UK). Primers are given in Supplemental Table 4 online. The difference between the resulting $\mathrm{C}(\mathrm{t})$ values of wt and Pro35S:HA-bZIP1 overexpressor was calculated and normalized with the input controls of these samples which were analysed with the same primers. For further normalization, PCR was performed with unspecific actin (ACT7) promoter primers (Table S4).

\section{SUPPLEMENTAL REFERENCES}

Hruz, T., Laule, O., Szabo, G., Wessendorp, F., Bleuler, S., Oertle, L., Widmayer, P., Gruissem, W. and Zimmermann, P. (2008). Genevestigator V3: a reference expression database for the metaanalysis of transcriptomes. Advances in Bioinformatics. 420747.

Kirby, J., and Kavanagh, T.A. (2002). NAN fusions: a synthetic sialidase reporter gene as a sensitive and versatile partner for GUS. Plant J. 32, 391- 400.

Onate-Sanchez, L., and Singh, K.B. (2002). Identification of Arabidopsis ethylene-responsive element binding factors with distinct induction kinetics after pathogen infection. Plant Physiol. 128, 1313-1322.

Pilot, G., Stransky, H., Bushey, D.F., Pratelli, R., Ludewig, U., Wingate, V.P., and Frommer, W.B. (2004). Overexpression of GLUTAMINE DUMPER1 leads to hypersecretion of glutamine from Hydathodes of Arabidopsis leaves. Plant Cell 16, 1827-1840.

\begin{tabular}{|c|c|c|c|c|c|c|c|c|c|}
\hline [ng/mg dry weight] & Aspartic Acid & Threonine & Serine & Asparagine & Glutamic Acid & Glutamine & Glycine & Alanine & Citrulline \\
\hline wt 0d & 9,85 & 8,24 & 19,71 & 26,54 & 27,95 & 51,28 & 1,85 & 4,52 & 1,61 \\
\hline wt 1d & 9,21 & 7,75 & 11,95 & 39,24 & 24,06 & 53,13 & 0,68 & 5,75 & 2,20 \\
\hline wt 4d & 6,30 & 8,79 & 24,07 & 134,10 & 38,78 & 56,93 & 1,07 & 4,28 & 1,34 \\
\hline wt 6d & 17,17 & 13,03 & 55,64 & 368,79 & 104,60 & 54,05 & 0,99 & 5,15 & 1,11 \\
\hline Pro35S:bZIP1 0d & 6,32 & 5,88 & 15,63 & 17,13 & 27,93 & 20,11 & 1,43 & 3,13 & 0,79 \\
\hline Pro35S:bZIP1 1d & 5,14 & 8,28 & 15,74 & 38,60 & 46,39 & 21,87 & 1,13 & 5,33 & 1,82 \\
\hline Pro35S:bZIP1 4d & 3,92 & 3,77 & 20,66 & 105,24 & 87,30 & 14,78 & 2,32 & 3,26 & 0,63 \\
\hline Pro35S:bZIP1 6d & 5,98 & 4,56 & 35,33 & 163,39 & 86,61 & 20,09 & 1,71 & 3,73 & 0,68 \\
\hline Pro35S:bZIP53 0d & 7,53 & 11,06 & 19,05 & 119,08 & 28,89 & 57,77 & 9,88 & 3,18 & 3,46 \\
\hline Pro35S:bZIP53 1d & 8,94 & 6,14 & 9,12 & 93,08 & 26,25 & 32,58 & 1,06 & 4,24 & 1,34 \\
\hline Pro35S:bZIP53 4d & 10,42 & 16,45 & 35,65 & 504,61 & 72,40 & 80,08 & 1,14 & 1,93 & 2,37 \\
\hline Pro35S:bZIP53 6d & 10,50 & 14,18 & 30,98 & 536,72 & 82,98 & 63,02 & 1,30 & 1,60 & 2,33 \\
\hline bzip1 bzip53 0d & 4,38 & 3,53 & 12,17 & 10,83 & 16,19 & 47,71 & 1,19 & 5,11 & 0,39 \\
\hline bzip1 bzip53 1d & 7,40 & 4,52 & 7,55 & 13,30 & 14,14 & 37,40 & 1,07 & 6,90 & 0,77 \\
\hline bzip1 bzip53 4d & 1,98 & 3,08 & 7,89 & 46,23 & 11,63 & 16,86 & 0,72 & 5,28 & 0,63 \\
\hline bzip1 bzip53 6d & 3,49 & 7,54 & 19,96 & 113,07 & 31,83 & 43,41 & 0,78 & 5,22 & 0,50 \\
\hline [ng/mg dry weight] & Aminobutyric Acid & Valine & Cysteine & Met/Cysta & Isoleucine & Leucine & Tyrosine & Phenylalanine & GABA \\
\hline wt 0d & 1,81 & 1,85 & 0,00 & 0,52 & 0,95 & 0,99 & 0,70 & 0,72 & 0,52 \\
\hline wt 1d & 1,23 & 4,05 & 0,00 & 0,21 & 2,07 & 2,29 & 1,32 & 2,23 & 1,47 \\
\hline wt 4d & 2,90 & 23,19 & 0,00 & 0,00 & 10,62 & 6,53 & 4,05 & 22,81 & 0,73 \\
\hline wt 6d & 3,34 & 25,15 & 0,00 & 0,79 & 9,22 & 5,28 & 3,91 & 34,24 & 3,62 \\
\hline Pro35S:bZIP1 0d & 1,43 & 1,32 & 0,00 & 0,00 & 0,61 & 0,32 & 0,38 & 0,59 & 0,00 \\
\hline Pro35S:bZIP1 1d & 2,12 & 4,21 & 0,00 & 0,00 & 2,29 & 1,59 & 1,66 & 2,82 & 0,00 \\
\hline Pro35S:bZIP1 4d & 3,38 & 14,32 & 0,00 & 0,00 & 3,20 & 1,51 & 1,66 & 26,84 & 3,02 \\
\hline Pro35S:bZIP1 6d & 4,62 & 26,44 & 0,00 & 0,20 & 4,16 & 1,65 & 1,60 & 54,70 & 4,16 \\
\hline Pro35S:bZIP53 0d & 1,91 & 1,29 & 0,00 & 0,00 & 0,43 & 0,37 & 0,65 & 1,14 & 0,00 \\
\hline Pro35S:bZIP53 1d & 2,01 & 2,27 & 0,00 & 0,00 & 1,08 & 0,67 & 0,87 & 1,66 & 1,60 \\
\hline Pro35S:bZIP53 4d & 3,25 & 10,77 & 0,00 & 0,68 & 3,62 & 1,84 & 1,60 & 40,59 & 1,08 \\
\hline Pro35S:bZIP53 6d & 3,53 & 14,01 & 0,00 & 0,80 & 4,81 & 2,63 & 2,10 & 59,87 & 2,02 \\
\hline bzip1 bzip53 0d & 1,27 & 1,19 & 0,00 & 0,00 & 0,61 & 0,80 & 0,61 & 0,78 & 0,00 \\
\hline bzip1 bzip53 1d & 1,19 & 2,80 & 0,00 & 0,00 & 1,69 & 2,26 & 1,34 & 1,65 & 0,00 \\
\hline bzip1 bzip53 4d & 1,54 & 13,06 & 0,00 & 0,00 & 6,44 & 5,12 & 2,94 & 10,51 & 0,99 \\
\hline bzip1 bzip53 6d & 1,98 & 17,98 & 0,00 & 0,53 & 7,79 & 5,19 & 2,71 & 18,34 & 2,76 \\
\hline
\end{tabular}




\begin{tabular}{|c|c|c|c|c|c|c|c|c|}
\hline [ng/mg dry weight] & Tryptophane & Hydroxy Lysi & Ammonia & Ornithine & Lysine & Histidine & Arginine & Proline \\
\hline wt 0d & 0,00 & 0,70 & $\overline{8,06}$ & $\overline{1,01}$ & 1,37 & 0,68 & 23,53 & $\overline{2,63}$ \\
\hline wt 1d & 0,00 & 1,60 & 12,90 & 1,49 & 2,84 & 1,47 & 20,51 & 1,76 \\
\hline wt $4 d$ & 4,43 & 0,00 & 41,19 & 1,41 & 6,15 & 9,36 & 25,41 & 1,87 \\
\hline wt $6 \mathrm{~d}$ & 6,10 & 2,19 & 143,06 & 2,35 & 5,05 & 11,70 & 64,86 & 1,11 \\
\hline Pro35S:bZIP1 0d & 0,00 & 0,70 & 7,32 & 0,47 & 0,85 & 1,05 & 10,74 & 1,14 \\
\hline Pro35S:bZIP1 1d & 0,00 & 0,00 & 11,27 & 0,63 & 2,42 & 2,19 & 10,83 & 1,13 \\
\hline Pro35S:bZIP1 4d & 6,69 & 2,62 & 96,65 & 1,15 & 2,50 & 9,05 & 31,66 & 1,00 \\
\hline Pro35S:bZIP1 6d & 11,28 & 0,40 & 202,42 & 0,00 & 1,11 & 16,24 & 35,04 & 1,42 \\
\hline Pro35S:bZIP53 0d & 0,00 & 1,31 & 12,35 & 2,43 & 1,11 & 1,58 & 35,34 & 0,38 \\
\hline Pro35S:bZIP53 1d & 0,00 & 2,20 & 8,56 & 1,04 & 1,62 & 1,79 & 15,45 & 0,56 \\
\hline Pro35S:bZIP53 4d & 4,80 & 0,66 & 70,21 & 3,27 & 5,40 & 0,00 & 110,80 & 0,37 \\
\hline Pro35S:bZIP53 6d & 8,51 & 0,86 & 73,52 & 4,71 & 6,91 & 25,73 & 141,27 & 0,97 \\
\hline bzip1 bzip53 0d & 0,00 & 0,00 & 4,65 & 0,66 & 0,68 & 0,71 & 10,05 & 1,87 \\
\hline bzip1 bzip53 1d & 0,00 & 0,00 & 3,49 & 0,00 & 2,53 & 1,49 & 7,97 & 1,76 \\
\hline bzip1 bzip53 4d & 3,38 & 2,70 & 28,96 & 1,71 & 5,56 & 4,90 & 19,80 & 1,40 \\
\hline $536 \mathrm{~d}$ & 3,04 & 0,00 & 34,00 & 1,23 & 4,91 & 7,54 & 28,62 & 20 \\
\hline
\end{tabular}

\begin{tabular}{|l|c|}
\hline [ng / mg dry weight] & total amino acids \\
\hline wt 0d & 151,56 \\
wt 1d & 153,97 \\
wt 4d & 275,65 \\
wt 6d & 620,52 \\
\hline Pro35S:bZIP1 0d & 98,37 \\
Pro35S:bZIP1 1d & 144,30 \\
Pro35S:bZIP1 4d & 241,88 \\
Pro35S:bZIP1 6d & 322,08 \\
\hline Pro35S:bZIP53 0d & 259,91 \\
Pro35S:bZIP53 1d & 182,75 \\
Pro35S:bZIP53 4d & 725,06 \\
Pro35S:bZIP53 6d & 743,62 \\
\hline bzip1 bzip53 0d & 101,50 \\
bzip1 bzip53 1d & 93,30 \\
bzip1 bzip53 4d & 94,30 \\
bzip1 bzip53 6d & 225,80 \\
\hline
\end{tabular}

\begin{tabular}{|c|c|c|c|c|c|c|c|c|c|}
\hline experiment 2 & & & & & & & & & \\
\hline [ng/mg dry weight] & Aspartic Acid & Threonine & Serine & Asparagine & Glutamic Acid & Glutamine & Glycine & Alanine & Citrulline \\
\hline wt 0d & 8,74 & 8,98 & 15,30 & 11,90 & 34,48 & 25,49 & 3,42 & 3,74 & 2,62 \\
\hline wt 1d & 14,11 & 13,83 & 21,30 & 50,07 & 47,30 & 68,88 & 1,33 & 10,35 & 2,35 \\
\hline wt 4d & 6,42 & 10,71 & 21,84 & 152,03 & 55,03 & 26,34 & 1,22 & 5,18 & 1,13 \\
\hline wt $6 \mathrm{~d}$ & 5,88 & 8,94 & 29,41 & 192,93 & 66,59 & 30,12 & 2,16 & 5,76 & 0,89 \\
\hline Pro35S:bZIP1 0d & 4,65 & 3,64 & 8,47 & 15,03 & 18,08 & 14,62 & 1,78 & 3,46 & 1,50 \\
\hline Pro35S:bZIP1 1d & 6,06 & 6,88 & 12,71 & 34,40 & 46,44 & 27,59 & 0,85 & 4,41 & 1,15 \\
\hline Pro35S:bZIP1 4d & 3,96 & 5,32 & 27,23 & 138,29 & 82,15 & 20,69 & 1,89 & 2,49 & 0,37 \\
\hline Pro35S:bZIP1 6d & 3,99 & 2,74 & 17,95 & 109,18 & 69,92 & 13,34 & 1,05 & 2,19 & 0,42 \\
\hline Pro35S:bZIP53 0d & 11,33 & 14,09 & 23,89 & 118,53 & 45,63 & 67,69 & 8,15 & 3,61 & 3,64 \\
\hline Pro35S:bZIP53 1d & 8,28 & 7,81 & 9,69 & 103,75 & 27,81 & 61,72 & 1,06 & 4,81 & 1,81 \\
\hline Pro35S:bZIP53 4d & 11,12 & 13,65 & 30,84 & 495,97 & 79,88 & 57,13 & 1,01 & 2,22 & 1,56 \\
\hline Pro35S:bZIP53 6d & 12,45 & 8,47 & 29,88 & 611,54 & 91,13 & 64,74 & 1,00 & 1,12 & 1,97 \\
\hline bzip1 bzip53 0d & 5,35 & 5,17 & 18,01 & 13,37 & 25,31 & 47,78 & 3,32 & 5,10 & 0,93 \\
\hline bzip1 bzip53 1d & 3,01 & 2,03 & 4,04 & 9,08 & 7,93 & 18,54 & 0,68 & 5,95 & 1,83 \\
\hline bzip1 bzip53 4d & 1,79 & 3,78 & 7,98 & 45,62 & 12,85 & 20,75 & 0,75 & 6,24 & 0,75 \\
\hline bzip1 bzip53 6d & 3,67 & 7,17 & 21,33 & 105,50 & 34,83 & 49,00 & 0,73 & 6,50 & 0,27 \\
\hline [ng/mg dry weight] & Aminobutyric Acid & Valine & Cysteine & Met/Cysta & Isoleucine & Leucine & Tyrosine & Phenylalanine & GABA \\
\hline wt Od & 2,33 & 2,14 & 0,00 & 0,63 & 0,78 & 0,68 & 0,00 & 0,68 & 0,00 \\
\hline wt 1d & 1,99 & 6,50 & 0,00 & 0,00 & 3,43 & 3,76 & 2,10 & 2,07 & 1,27 \\
\hline wt 4d & 3,15 & 20,15 & 0,00 & 0,15 & 8,29 & 6,06 & 2,76 & 22,98 & 1,78 \\
\hline wt $6 \mathrm{~d}$ & 3,46 & 29,36 & 0,00 & 1,11 & 10,63 & 6,33 & 4,35 & 40,21 & 4,71 \\
\hline Pro35S:bZIP1 0d & 2,46 & 1,82 & 0,00 & 0,00 & 0,00 & 0,00 & 0,00 & 1,18 & 0,00 \\
\hline Pro35S:bZIP1 1d & 2,04 & 3,81 & 0,00 & 0,00 & 1,62 & 1,10 & 1,74 & 2,22 & 0,00 \\
\hline Pro35S:bZIP1 4d & 3,44 & 15,97 & 0,00 & 0,00 & 4,23 & 2,16 & 2,28 & 27,84 & 2,95 \\
\hline Pro35S:bZIP1 6d & 2,37 & 11,14 & 0,00 & 0,52 & 2,44 & 1,12 & 1,00 & 33,28 & 1,99 \\
\hline Pro35S:bZIP53 Od & 3,71 & 2,42 & 0,00 & 0,00 & 1,10 & 0,92 & 0,98 & 2,51 & 1,78 \\
\hline Pro35S:bZIP53 1d & 1,86 & 2,09 & 0,00 & 0,00 & 1,11 & 0,80 & 0,86 & 1,94 & 1,13 \\
\hline Pro35S:bZIP53 4d & 3,22 & 11,97 & 0,00 & 0,79 & 3,92 & 1,94 & 1,38 & 45,50 & 1,48 \\
\hline Pro35S:bZIP53 6d & 3,21 & 10,92 & 0,00 & 0,72 & 3,90 & 1,87 & 2,09 & 61,25 & 1,79 \\
\hline bzip1 bzip53 0d & 1,93 & 1,14 & 0,00 & 0,00 & 0,00 & 0,00 & 0,00 & 0,00 & 0,00 \\
\hline bzip1 bzip53 1d & 1,33 & 2,74 & 0,00 & 0,00 & 1,41 & 2,15 & 1,12 & 1,18 & 0,00 \\
\hline bzip1 bzip53 4d & 2,29 & 15,01 & 0,00 & 0,00 & 7,11 & 5,41 & 2,87 & 13,35 & 2,16 \\
\hline bzip1 bzip53 6d & 1,73 & 18,40 & 0,00 & 0,00 & 8,13 & 5,27 & 2,77 & 18,80 & 2,53 \\
\hline
\end{tabular}




\begin{tabular}{|c|c|c|c|c|c|c|c|c|}
\hline [ng / mg dry weight] & Tryptophane & Hydroxy Lysi & Ammonia & Ornithine & Lysine & Histidine & Arginine & Proline \\
\hline wt 0d & 0,00 & 2,14 & $\overline{6,34}$ & 0,56 & 0,61 & 0,00 & 4,73 & 2,72 \\
\hline wt 1d & 0,00 & 0,47 & 11,40 & 1,02 & 3,96 & 1,91 & 13,25 & 1,49 \\
\hline wt 4d & 3,17 & 1,67 & 57,32 & 1,24 & 5,20 & 8,20 & 36,19 & 1,88 \\
\hline wt 6d & 6,49 & 0,00 & 101,88 & 1,62 & 5,11 & 13,60 & 36,70 & 1,44 \\
\hline Pro35S:bZIP1 0d & 0,00 & 0,00 & 6,79 & 0,87 & 1,23 & 1,59 & 11,89 & 1,91 \\
\hline Pro35S:bZIP1 1d & 0,00 & 2,49 & 25,23 & 0,97 & 2,47 & 1,70 & 12,26 & 1,15 \\
\hline Pro35S:bZIP1 4d & 7,09 & 1,52 & 101,01 & 0,00 & 2,13 & 12,32 & 25,86 & 1,25 \\
\hline Pro35S:bZIP1 6d & 4,61 & 0,00 & 115,29 & 0,00 & 0,90 & 12,21 & 22,81 & 0,95 \\
\hline Pro35S:bZIP53 0d & 0,00 & 1,04 & 5,67 & 1,68 & 1,41 & 2,39 & 38,90 & 0,46 \\
\hline Pro35S:bZIP53 1d & 0,00 & 1,05 & 16,09 & 2,23 & 1,84 & 1,95 & 32,03 & 0,63 \\
\hline Pro35S:bZIP53 4d & 5,50 & 1,27 & 36,91 & 2,79 & 5,48 & 11,97 & 99,60 & 0,75 \\
\hline Pro35S:bZIP53 6d & 8,61 & 0,48 & 67,73 & 3,23 & 6,18 & 31,37 & 126,99 & 0,86 \\
\hline bzip1 bzip53 0d & 0,00 & 0,00 & 12,02 & 0,53 & 0,82 & 0,71 & 6,77 & n.d. \\
\hline bzip1 bzip53 1d & 0,00 & 0,00 & 8,05 & 0,56 & 1,36 & 0,85 & 8,37 & 1,86 \\
\hline bzip1 bzip53 4d & 2,95 & 0,00 & 26,70 & 1,62 & 5,82 & 5,82 & 24,62 & 2,12 \\
\hline bzip1 bzip53 6d & 4,17 & 0,00 & 41,17 & 1,43 & 5,23 & 7,17 & 32,17 & 1,40 \\
\hline
\end{tabular}

\begin{tabular}{|l|c|}
\hline [ng / mg dry weight] & total amino acids \\
\hline wt 0d & 114,67 \\
wt 1d & 229,5 \\
wt 4d & 279,9 \\
wt 6d & 342,69 \\
\hline Pro35S:bZIP1 0d & 71,23 \\
Pro35S:bZIP1 1d & 140,49 \\
Pro35S:bZIP1 4d & 282,38 \\
Pro35S:bZIP1 6d & 220,78 \\
\hline Pro35S:bZIP53 0d & 296,56 \\
Pro35S:bZIP53 1d & 226,75 \\
Pro35S:bZIP53 4d & 693,39 \\
Pro35S:bZIP53 6d & 822,3 \\
\hline bzip1 bzip53 0d & 124,33 \\
bzip1 bzip53 1d & 53,08 \\
bzip1 bzip53 4d & 100,51 \\
bzip1 bzip53 6d & 229 \\
\hline
\end{tabular}

\begin{tabular}{|c|c|c|c|c|c|c|c|c|c|c|}
\hline \multicolumn{11}{|c|}{ or for eac } \\
\hline$[\mathrm{ng} / \mathrm{mg}$ dry weight] & Aspartic Acid & & Threonine & & Serine & & Asparagine & & Glutamic A & \\
\hline & Mean & $\mathrm{SD}$ & Mean & $\mathrm{SD}$ & Mean & $\overline{\mathrm{SD}}$ & Mean & $\overline{\mathrm{SD}}$ & Mean & $\overline{S D}$ \\
\hline wt 0d & 9,30 & 0,79 & 8,61 & 0,52 & 17,50 & 3,12 & 19,22 & 10,36 & 18,04 & 4,61 \\
\hline wt 1d & 11,66 & 3,47 & 10,79 & 4,30 & 16,62 & 6,61 & 44,66 & 7,65 & 24,75 & 16,43 \\
\hline wt 4d & 6,36 & 0,08 & 9,75 & 1,36 & 22,95 & 1,58 & 143,06 & 12,67 & 28,18 & 11,49 \\
\hline wt 6d & 11,52 & 7,98 & 10,99 & 2,89 & 42,52 & 18,54 & 280,86 & 124,35 & 33,85 & 26,88 \\
\hline Pro35S:bZIP1 0d & 5,48 & 1,19 & 4,76 & 1,58 & 12,05 & 5,06 & 16,08 & 1,48 & 9,44 & 6,96 \\
\hline Pro35S:bZIP1 1d & 5,60 & 0,65 & 7,58 & 0,99 & 14,23 & 2,14 & 36,50 & 2,97 & 24,13 & 0,04 \\
\hline Pro35S:bZIP1 4d & 3,94 & 0,02 & 4,55 & 1,10 & 23,94 & 4,65 & 121,76 & 23,36 & 41,39 & 3,64 \\
\hline Pro35S:bZIP1 6d & 4,99 & 1,41 & 3,65 & 1,28 & 26,64 & 12,29 & 136,29 & 38,33 & 35,30 & 11,80 \\
\hline Pro35S:bZIP53 Od & 9,43 & 2,69 & 12,58 & 2,14 & 21,47 & 3,42 & 118,80 & 0,39 & 24,55 & 11,84 \\
\hline Pro35S:bZIP53 1d & 8,61 & 0,46 & 6,98 & 1,18 & 9,40 & 0,40 & 98,41 & 7,55 & 14,58 & 1,11 \\
\hline Pro35S:bZIP53 4d & 10,77 & 0,50 & 15,05 & 1,98 & 33,25 & 3,40 & 500,29 & 6,11 & 41,13 & 5,29 \\
\hline Pro35S:bZIP53 6d & 11.48 & 1,38 & 11,32 & 4.04 & 30,43 & 0,78 & 574,13 & 5291 & 46,73 & 5,77 \\
\hline bzip1 bzip53 0d & 4,86 & 0,68 & 4,35 & 1,16 & 15,09 & 4,13 & 12,10 & 1,80 & 12,85 & 6,46 \\
\hline bzip1 bzip53 1d & 5,20 & 3,10 & 3,28 & 1,76 & 5,79 & 2,48 & 11,19 & 2,98 & 4,35 & 4,39 \\
\hline bzip 1 bzip53 4d & 1,88 & 0,14 & 3,43 & 0,50 & 7,94 & 0,06 & 45,92 & 0,43 & 6,74 & 0,86 \\
\hline & & & & & & & & & & \\
\hline
\end{tabular}

\begin{tabular}{|c|c|c|c|c|c|c|c|c|c|c|}
\hline & Glutamine & & Glycine & & Alanine & & Citrulline & & Aminobut & \\
\hline & Mean & $\mathrm{SD}$ & Mean & $\mathrm{SD}$ & Mean & $\mathrm{SD}$ & Mean & $\mathrm{SD}$ & Mean & $\mathrm{SD}$ \\
\hline wt od & 38,38 & 18,23 & 2,64 & 1,11 & 4,13 & 0,56 & 2,12 & 0,72 & 2,07 & 0,37 \\
\hline wt 1d & 61,00 & 11,13 & 1,00 & 0,46 & 8,05 & 3,25 & 2,27 & 0,11 & 1,61 & 0,54 \\
\hline wt 4d & 41,63 & 21,63 & 1,15 & 0,11 & 4,73 & 0,64 & 1,24 & 0,14 & 3,03 & 0,17 \\
\hline wt $6 d$ & 42,08 & 16,92 & 1,58 & 0,83 & 5,46 & 0,43 & 1,00 & 0,15 & 3,40 & 0,09 \\
\hline Pro35S:bZIP1 0d & 17,37 & 3,88 & 1,61 & 0,24 & 3,30 & 0,23 & 1,15 & 0,50 & 1,95 & 0,72 \\
\hline Pro35S:bZIP1 1d & 24,73 & 4,05 & 0,99 & 0,20 & 4,87 & 0,65 & 1,48 & 0,48 & 2,08 & 0,05 \\
\hline Pro35S:bZIP1 4d & 17,73 & 4,18 & 2,10 & 0,31 & 2,88 & 0,54 & 0,50 & 0,19 & 3,41 & 0,04 \\
\hline Pro35S:bZIP1 6d & 16,71 & 4,77 & 1,38 & 0,47 & 2,96 & 1,09 & 0,55 & 0,18 & 3,49 & 1,59 \\
\hline Pro35S:bZIP53 0d & 62,73 & 7,01 & 9,01 & 1,23 & 3,40 & 0,31 & 3,55 & 0,13 & 2,81 & 1,27 \\
\hline Pro35S:bZIP53 1d & 47,15 & 20,61 & 1,06 & 0,00 & 4,53 & 0,40 & 1,58 & 0,33 & 1,93 & 0,11 \\
\hline Pro35S:bZIP53 4d & 68,60 & 16,23 & 1,08 & 0,09 & 2,08 & 0,21 & 1,96 & 0,57 & 3,23 & 0,02 \\
\hline Pro35S:bZIP53 6d & 63,88 & 1,22 & 1,15 & 0,22 & 1,36 & 0,34 & 2,15 & 0,25 & 3,37 & 0,23 \\
\hline bzip1 bzip53 0d & 47,74 & 0,05 & 2,25 & 1,50 & 5,10 & 0,01 & 0,66 & 0,38 & 1,60 & 0,47 \\
\hline bzip1 bzip53 1d & 27,97 & 13,34 & 0,88 & 0,28 & 6,43 & 0,67 & 1,30 & 0,75 & 1,26 & 0,10 \\
\hline bzip1 bzip53 4d & 18,80 & 2,75 & 0,73 & 0,02 & 5,76 & 0,68 & 0,69 & 0,08 & 1,91 & 0,53 \\
\hline bzip1 bzip53 6d & 46,21 & 3,95 & 0,76 & 0,03 & 5,86 & 0,90 & 0,38 & 0,17 & 1,86 & 0,18 \\
\hline
\end{tabular}




\begin{tabular}{|c|c|c|c|c|c|c|c|c|c|c|}
\hline & \multicolumn{2}{|l|}{ Valine } & \multicolumn{2}{|l|}{ Cysteine } & \multicolumn{2}{|l|}{ Met/Cysta } & \multicolumn{2}{|l|}{ Isoleucine } & \multicolumn{2}{|l|}{ Leucine } \\
\hline & Mean & $\mathrm{SD}$ & Mean & $\mathrm{SD}$ & Mean & $\mathrm{SD}$ & Mean & $\mathrm{SD}$ & Mean & $\mathrm{SD}$ \\
\hline wt 0d & 1,99 & 0,20 & 0,00 & 0,00 & 0,58 & 0,08 & 0,86 & 0,12 & 0,83 & 0,22 \\
\hline wt 1d & 5,28 & 1,73 & 0,00 & 0,00 & 0,10 & 0,15 & 2,75 & 0,96 & 3,03 & 1,04 \\
\hline wt 4d & 21,67 & 2,15 & 0,00 & 0,00 & 0,07 & 0,11 & 9,45 & 1,65 & 6,30 & 0,33 \\
\hline$w t 6 d$ & 27,26 & 2,98 & 0,00 & 0,00 & 0,95 & 0,22 & 9,93 & 1,00 & 5,80 & 0,74 \\
\hline Pro35s:bZIP1 0d & 1,57 & 0,36 & 0,00 & 0,00 & 0,00 & 0,00 & 0,31 & 0,43 & 0,16 & 0,23 \\
\hline Pro35S:bZIP1 1d & 4,01 & 0,28 & 0,00 & 0,00 & 0,00 & 0,00 & 1,95 & 0,47 & 1,34 & 0,35 \\
\hline Pro35S:bZIP1 4d & 15,15 & 1,17 & 0,00 & 0,00 & 0,00 & 0,00 & 3,71 & 0,73 & 1,83 & 0,46 \\
\hline Pro35S:bZIP1 6d & 18,79 & 10,82 & 0,00 & 0,00 & 0,36 & 0,23 & 3,30 & 1,21 & 1,39 & 0,38 \\
\hline Pro35S:bZIP53 0d & 1,86 & 0,80 & 0,00 & 0,00 & 0,00 & 0,00 & 0,77 & 0,48 & 0,64 & 0,39 \\
\hline Pro35S:bZIP53 1d & 2,18 & 0,13 & 0,00 & 0,00 & 0,00 & 0,00 & 1,09 & 0,02 & 0,73 & 0,09 \\
\hline Pro35S:bZIP53 4d & 11,37 & 0,85 & 0,00 & 0,00 & 0,73 & 0,08 & 3,77 & 0,21 & 1,89 & 0,07 \\
\hline Pro35S:bZIP53 6d & 12,46 & 2,19 & 0,00 & 0,00 & 0,76 & 0,06 & 4,36 & 0,64 & 2,25 & 0,53 \\
\hline bzip1 bzip53 0d & 1,17 & 0,04 & 0,00 & 0,00 & 0,00 & 0,00 & 0,30 & 0,43 & 0,40 & 0,57 \\
\hline bzip1 bzip53 1d & 2,77 & 0,04 & 0,00 & 0,00 & 0,00 & 0,00 & 1,55 & 0,19 & 2,21 & 0,08 \\
\hline bzip1 bzip53 4d & 14,04 & 1,38 & 0,00 & 0,00 & 0,00 & 0,00 & 6,77 & 0,48 & 5,26 & 0,21 \\
\hline \multirow[t]{3}{*}{ bzip1 bzip53 6d } & 18,19 & 0,30 & 0,00 & 0,00 & 0,27 & 0,38 & 7,96 & 0,24 & 5,23 & 0,05 \\
\hline & Tyrosine & & Phenylala & & $\overline{G A B A}$ & & Tryptopha & & Hydroxy L & \\
\hline & Mean & $\overline{\mathrm{SD}}$ & Mean & $\overline{S D}$ & Mean & $\mathrm{SD}$ & Mean & $\mathrm{SD}$ & Mean & $\mathrm{SD}$ \\
\hline wt 0d & 0,35 & 0,50 & 0,70 & 0,03 & 0,26 & 0,37 & 0,00 & 0,00 & 1,42 & 1,01 \\
\hline wt 1d & 1,71 & 0,55 & 2,15 & 0,11 & 1,37 & 0,14 & 0,00 & 0,00 & 1,03 & 0,80 \\
\hline wt $4 d$ & 3,41 & 0,91 & 22,89 & 0,12 & 1,25 & 0,74 & 3,80 & 0,89 & 0,84 & 1,18 \\
\hline wt $6 d$ & 4,13 & 0,31 & 37,23 & 4,22 & 4,16 & 0,76 & 6,30 & 0,28 & 1,10 & 1,55 \\
\hline Pro35S:bZIP1 0d & 0,19 & 0,27 & 0,88 & 0,42 & 0,00 & 0,00 & 0,00 & 0,00 & 0,35 & 0,50 \\
\hline Pro35S:bZIP1 1d & 1,70 & 0,06 & 2,52 & 0,42 & 0,00 & 0,00 & 0,00 & 0,00 & 1,25 & 1,76 \\
\hline Pro35S:bZIP1 4d & 1,97 & 0,44 & 27,34 & 0,71 & 2,98 & 0,05 & 6,89 & 0,28 & 2,07 & 0,78 \\
\hline Pro35S:bZIP1 6d & 1,30 & 0,42 & 43,99 & 15,15 & 3,08 & 1,53 & 7,95 & 4,72 & 0,20 & 0,28 \\
\hline Pro35S:bZIP53 0d & 0,81 & 0,24 & 1,82 & 0,97 & 0,89 & 1,26 & 0,00 & 0,00 & 1,17 & 0,19 \\
\hline Pro35S:bZIP53 1d & 0,87 & 0,01 & 1,80 & 0,20 & 1,36 & 0,34 & 0,00 & 0,00 & 1,62 & 0,81 \\
\hline Pro35S:bZIP53 4d & 1,49 & 0,16 & 43,05 & 3,47 & 1,28 & 0,28 & 5,15 & 0,49 & 0,97 & 0,44 \\
\hline Pro35S:bZIP53 6d & 2,10 & 0,01 & 60,56 & 0,98 & 1,90 & 0,16 & 8,56 & 0,07 & 0,67 & 0,27 \\
\hline bzip1 bzip53 0d & 0,30 & 0,43 & 0,39 & 0,55 & 0,00 & 0,00 & 0,00 & 0,00 & 0,00 & 0,00 \\
\hline bzip1 bzip53 1d & 1,23 & 0,16 & 1,41 & 0,33 & 0,00 & 0,00 & 0,00 & 0,00 & 0,00 & 0,00 \\
\hline bzip1 bzip53 4d & 2,91 & 0,05 & 11,93 & 2,01 & 1,58 & 0,83 & 3,17 & 0,30 & 1,35 & 1,91 \\
\hline bzip1 bzip53 6d & 2,74 & 0,04 & 18,57 & 0,32 & 2,65 & 0,16 & 3,60 & 0,79 & 0,00 & 0,00 \\
\hline
\end{tabular}

\begin{tabular}{|c|c|c|c|c|c|c|c|c|c|c|}
\hline & Ammonia & & Ornithine & & Lysine & & Histidine & & Arginine & \\
\hline & Mean & SD & Mean & $\mathrm{SD}$ & Mean & $\mathrm{SD}$ & Mean & $\mathrm{SD}$ & Mean & SD \\
\hline wt 0d & 7,20 & 1,22 & 0,78 & 0,32 & 0,99 & 0,54 & 0,34 & 0,48 & 14,13 & 13,29 \\
\hline wt 1d & 12,15 & 1,07 & 1,25 & 0,33 & 3,40 & 0,79 & 1,69 & 0,31 & 16,88 & 5,13 \\
\hline wt 4d & 49,25 & 11,41 & 1,33 & 0,12 & 5,68 & 0,67 & 8,78 & 0,82 & 30,80 & 7,62 \\
\hline wt $6 \mathrm{~d}$ & 122,47 & 29,12 & 1,99 & 0,52 & 5,08 & 0,04 & 12,65 & 1,34 & 50,78 & 19,91 \\
\hline Pro35S:bZIP1 0d & 7,05 & 0,38 & 0,67 & 0,28 & 1,04 & 0,27 & 1,32 & 0,38 & 11,32 & 0,81 \\
\hline Pro35S:bZIP1 1d & 18,25 & 9,87 & 0,80 & 0,24 & 2,44 & 0,03 & 1,94 & 0,35 & 11,55 & 1,01 \\
\hline Pro35S:bZIP1 4d & 98,83 & 3,09 & 0,57 & 0,81 & 2,32 & 0,26 & 10,68 & 2,32 & 28,76 & 4,10 \\
\hline Pro35s:bZIP1 6d & 158,86 & 61,61 & 0,00 & 0,00 & 1,00 & 0,15 & 14,23 & 2,85 & 28,93 & 8,65 \\
\hline Pro35S:bZIP53 0d & 9,01 & 4,73 & 2,06 & 0,53 & 1,26 & 0,21 & 1,99 & 0,57 & 37,12 & 2,51 \\
\hline Pro35S:bZIP53 1d & 12,33 & 5,32 & 1,64 & 0,84 & 1,73 & 0,16 & 1,87 & 0,12 & 23,74 & 11,72 \\
\hline Pro35S:bZIP53 4d & 53,56 & 23,55 & 3,03 & 0,34 & 5,44 & 0,06 & 5,99 & 8,47 & 105,20 & 7,92 \\
\hline Pro35S:bZIP53 6d & 70,63 & 4,10 & 3,97 & 1,05 & 6,54 & 0,52 & 28,55 & 3,99 & 134,13 & 10,10 \\
\hline bzip1 bzip53 0d & 8,33 & 5,21 & 0,60 & 0,09 & 0,75 & 0,10 & 0,71 & 0,01 & 8,41 & 2,32 \\
\hline bzip1 bzip53 1d & 5,77 & 3,22 & 0,28 & 0,40 & 1,94 & 0,83 & 1,17 & 0,45 & 8,17 & 0,28 \\
\hline bzip1 bzip53 4d & 27,83 & 1,60 & 1,66 & 0,06 & 5,69 & 0,19 & 5,36 & 0,66 & 22,21 & 3,41 \\
\hline bzip1 bzip53 6d & 37,59 & 5,06 & 1,33 & 0,14 & 5,07 & 0,23 & 7,35 & 0,26 & 30,39 & 2,51 \\
\hline
\end{tabular}

\begin{tabular}{|c|c|c|c|c|}
\hline & \multicolumn{2}{|l|}{ Proline } & \multicolumn{2}{|c|}{ total amino acid content } \\
\hline & Mean & $\mathrm{SD}$ & Mean & $\mathrm{SD}$ \\
\hline wt 0d & 2,68 & 0,06 & 168,30 & 41,45 \\
\hline wt 1d & 1,63 & 0,19 & 247,78 & 51,42 \\
\hline wt 4d & 1,88 & 0,01 & 448,20 & 16,81 \\
\hline wt $6 d$ & 1,27 & 0,23 & 776,10 & 235,34 \\
\hline Pro35S:bZIP1 0d & 1,53 & 0,55 & 113,14 & 17,21 \\
\hline Pro35S:bZIP1 1d & 1,14 & 0,01 & 193,37 & 8,38 \\
\hline Pro35S:bZIP1 4d & 1,12 & 0,18 & 469,78 & 32,05 \\
\hline Pro35S:bZIP1 6d & 1,19 & 0,34 & 559,47 & 181,09 \\
\hline Pro35S:bZIP53 0d & 0,42 & 0,05 & 340,85 & 29,23 \\
\hline Pro35S:bZIP53 1d & 0,59 & 0,05 & 258,24 & 48,25 \\
\hline Pro35S:bZIP53 4d & 0,56 & 0,27 & 955,92 & 39,69 \\
\hline Pro35S:bZIP53 6d & 0,91 & 0,08 & 1124,67 & 40,75 \\
\hline bzip1 bzip53 0d & 1,87 & & 136,81 & 16,18 \\
\hline bzip1 bzip53 1d & 1,81 & 0,07 & 102,63 & 26,27 \\
\hline bzip1 bzip53 4d & 1,76 & 0,51 & 210,83 & 10,66 \\
\hline bzip1 bzip53 6d & 130 & 014 & 371.50 & 1113 \\
\hline
\end{tabular}


Supplemental Data: ALONSO et al., (2009):

"A pivotal role of the basic leucine zipper transcription factor bZIP53 in the regulation of Arabidopsis seed maturation gene expression based on heterodimerisation and protein complex formation."

A

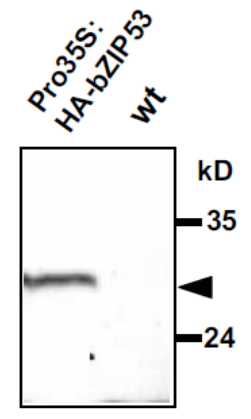

B

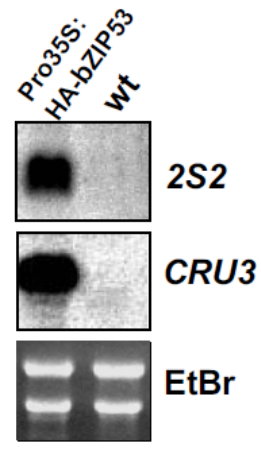

C
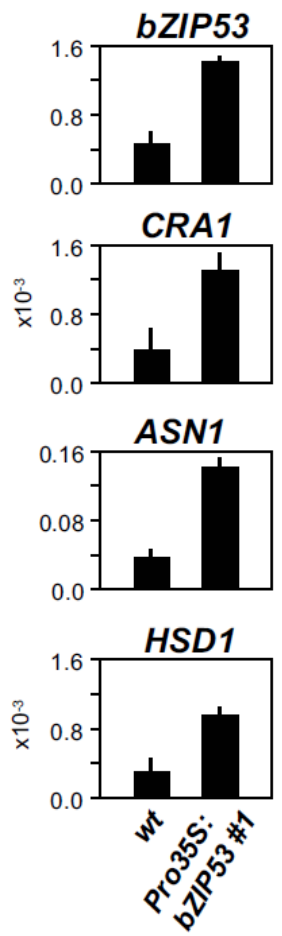

Supplemental Figure 1. Analysis of putative bZIP53 target genes in plants constitutively expressing HA-bZIP53 (A, B) or bZIP53 (C).

(A) Immunoblot analysis of Pro35S:HA-bZIP53 plants using a HA-tag-specific antiserum (_-HA) (arrow). Mass of marker proteins is provided in kilo Dalton $(\mathrm{kD})$.

(B) Transcription of 2S2 and CRU3 in leaves of wild-type (wt) and Pro35S:HA-bZIP53 plants has been studied by RNA gel blot analysis. Equal loading was confirmed by Ethidium bromide (EtBr) staining.

(C) RTqPCR analysis of two week-old wild-type (wt) and Pro35S:bZIP53 plants (line \#1) for expression of bZIP53, CRA1 (WANG et al., 2007), HSD1 (LI et al., 2007) and ASN1 (LAM et al., 2003). Expression levels are given as relative to a UBIQUITIN (UBI10) gene for normalization. Average values and standard errors from four replicates and two different experiments are shown. 
A

Effectors

\begin{tabular}{|r||c||c|}
\hline- & - & - \\
\hline bZIP53 & - & - \\
\hline- & bZIP10 & - \\
\hline bZIP53 & bZIP10 & - \\
\hline- & - & bZIP25 \\
\hline bZIP53 & - & bZIP25 \\
\hline
\end{tabular}

B

\begin{tabular}{|c|c|c|}
\hline \multicolumn{3}{|c|}{ Effectors } \\
\hline - & - & - \\
\hline bZIP53 & - & - \\
\hline- & bZIP10 & - \\
\hline bZIP53 & bZIP10 & - \\
\hline - & - & bZIP25 \\
\hline bZIP53 & - & bZIP25 \\
\hline
\end{tabular}

Reporter: ProCRU3 :GUS

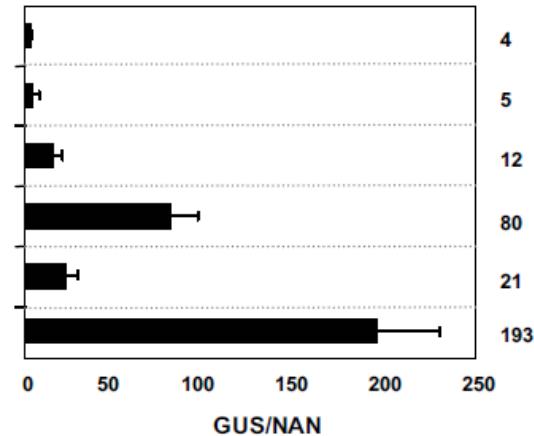

Reporter: Pro2S2:GUS

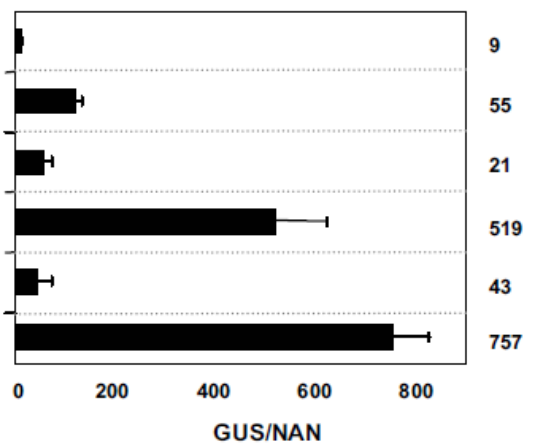

Suplemental Figure 2. Transient expression of bZIP53 in combination with bZIP10 or bZIP25 synergistically enhances transactivation of CRU3 and 2S2 promoters.

Arabidopsis protoplasts were transfected with constructs containing ProCRU3:GUS (A) or Pro2S2:GUS (B) reporters. Effector constructs containing specific bZIP genes under the control of a Pro35S were used in cotransfection experiments. $3 \mu \mathrm{g}$ of a control plasmid containing a Pro35S:NAN cassette was included in all experiments to normalize GUS expression values for differences in transfection efficiencies as described by EHLERT et al. (2006). X-axis values are expressed as GUS activity relative to NAN activity. Numbers along the Y-axis represent fold-induction values relative to non-transfected control cells. Given are mean values of 4 independent transfections. All experiments were repeated at least 3 times with similar results. 
A

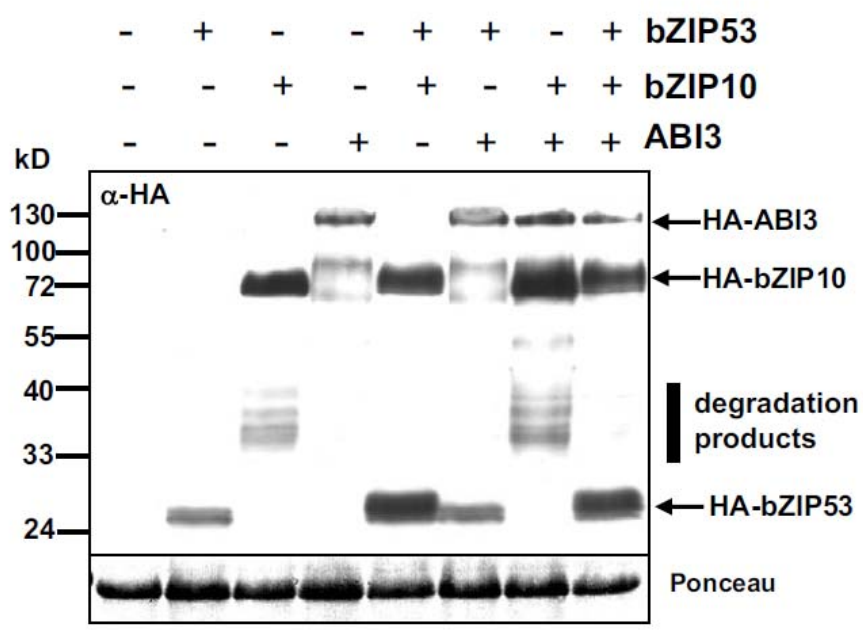

B
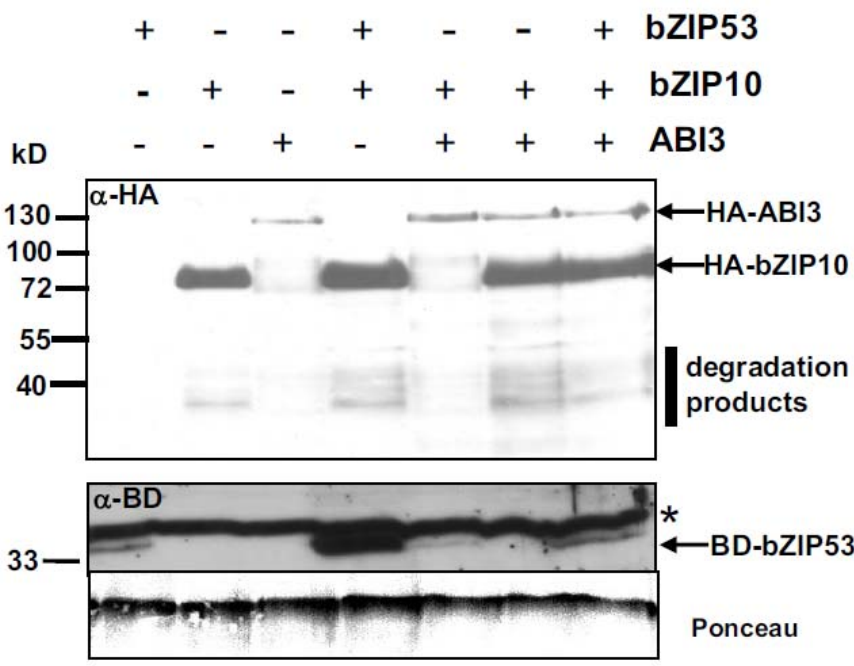

Supplemental Figure 3. Analysis of effector proteins transiently expressed in Arabidopsis protoplasts. Expression of the HA- and Gal4 DNA binding domain-(BD)- tagged bZIP effectors used in the experiments in Supplemental Figure 2 and Figure 6 (A) or Figure 4D (B), respectively. Proteins are detected in immunoblot experiments making use of _-HA or _-BD antisera. Protoplasts have been transiently transformed with the constructs indicated. Degradation products and tagged bZIP proteins are marked by a black line or arrows, respectively. Unspecific background staining is indicated by * Marker proteins are labelled according to their size in kilo Dalton (kD). Equal loading was confirmed by Ponceau staining. 


\section{$\beta$-Gal (Miller Units)}

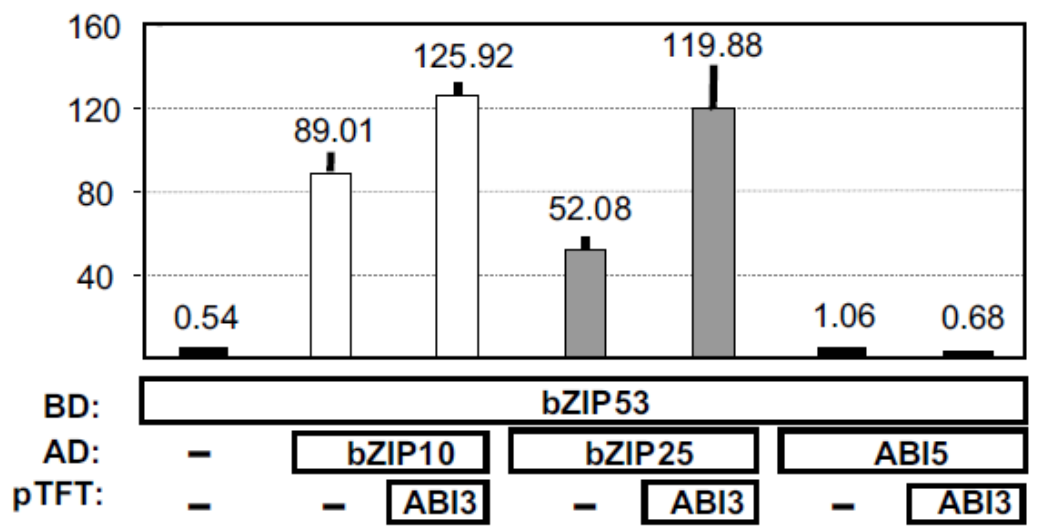

Supplemental Figure 4. Ternary protein interaction studied in a yeast three-hybrid system. Yeast strains (SFY526) expressing different combinations of BD-bZIP53, ADABI3 and bZIP10 or bZIP25 were assayed for _-galactosidase activity. The latter were provided in the three-hybrid vector pTFT (EGEA-CORTINES et al., 1999). Average values (Miller units, M.U.) and standard errors from six replicates and two independent experiments are shown.

\section{Reporter: Pro2S2 :GUS}

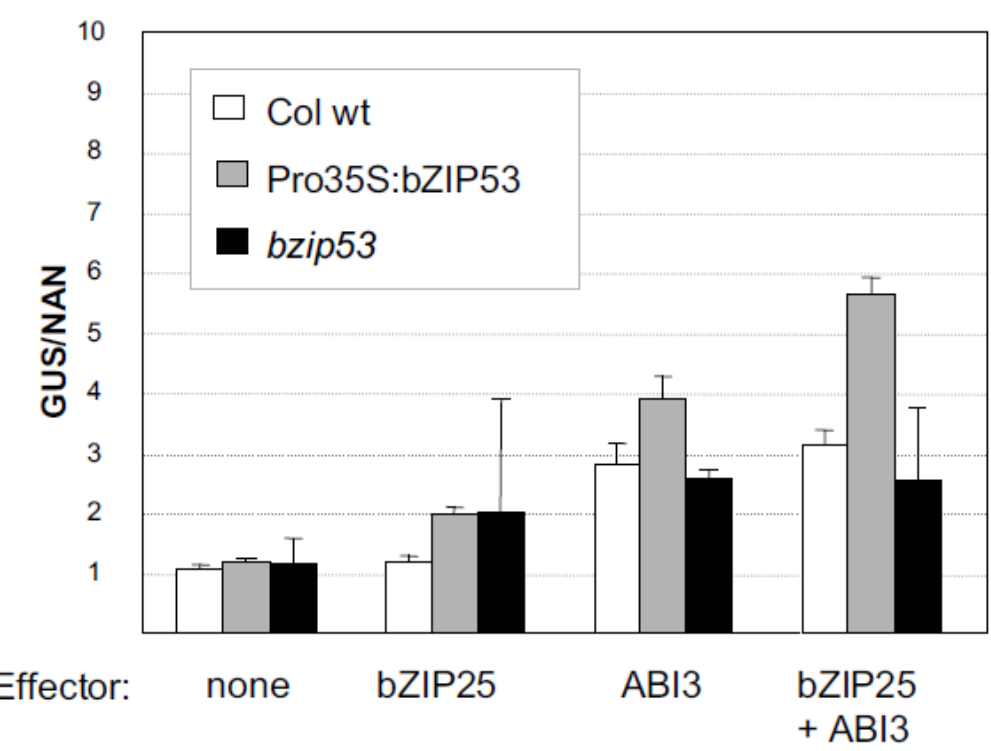

Supplemental Figure 5. Transient expression by microparticle bombardment of Arabidopsis leaves from Columbia wild type (Col wt; white bars) and plants with increased (Pro35S:bZIP53; grey bars) or decreased (bzip53; black bars) expression of bZIP53. Effector constructs containing bZIP25 or $\mathrm{ABI} 3$ under the control of a 35S promoter (Pro35S) and a GUS reporter gene under the control of an 2S2 promoter were used. X-axis values are expressed as GUS activity relative to NAN activity (KIRBY and KAVANAGH, 2002). Average values and standard errors from four replicates are shown. 
Supplemental Table 1: Oligonucleotide primers used in this study.

UBI:

5' - GCTCTTATCAAAGGACCTTCGG-3'

5' - CGAACTTGAGGAGGTTGCAAAG-3'

bZIP53

5'-TAATGATCCGAGGTACGCCAC-3'

5'-TGCTTCTGTTTCCTCATCCTTG-3'

CRU3:

5'-TAGATGTTCTCCAAGCCACCG-3'

5'-AACGGAAACACCAACACATCG-3'

2S2:

5' -ATTTGCAAGATCCAGCAAGTTG-3'

5'-AATACATTTAGCCTCAAACATC-3'

LEA76:

5'-ACAAAGAGCATTATCCAGGAAGT-3'

5' -ACACAAAGATACTTTCATATCGT-3'

ProDH:

5'-GCATCAAACGGTTCTGGTTTC-3'

5' -TGTTTATCGATCCCGAGGTCA-3'

ASN1:

5' -TTCAACGCCTTATGAGCCTCTT-3'

5' -CACCAGAGAGCAAAACTCCAAA-3'

CRA1:

5' -AGCCCAAATCCAGATCGTAAAC-3'

5'-TCACCACCGAGAAACCTTGTG-3'

HSD1:

5' -TGCCGGAAACAAAGATACGTG-3'

5'-AGTAACCGACAACCCCACTCA-3' 


\section{List of abbreviations}

\begin{tabular}{|c|c|}
\hline aa & amino acid \\
\hline ABA & abscisic acid \\
\hline $\mathrm{AD}$ & activation domain \\
\hline AMPK & AMP- activated protein kinase \\
\hline AKIN10/ 11 & Arabidopsis thaliana kinase $10 / 11$ \\
\hline Asn & amino acid asparagine \\
\hline ASN1 & asparagine synthetase gene \\
\hline At & Arabidopsis thaliana \\
\hline $\mathrm{BD}$ & DNA binding domain \\
\hline $\mathrm{BiFC}$ & bimolecular fluorescence completition \\
\hline bZIP & basic leucin Zipper \\
\hline ChIP & Chromatin Immuno Precipitation \\
\hline CPRF & Common Plant Regulatory Factor \\
\hline DNA & desoxy ribonuclein acid \\
\hline GCN4 & General Control Nondepressible \\
\hline LEA & late embryogenesis abundant \\
\hline LSD1 & Lesions Simulating Disease Resistance 1 \\
\hline MAT & maturation genes \\
\hline mRNA & messenger ribunuclein acid \\
\hline NLS & Nuclear Localisation Sequence \\
\hline $\mathrm{Nt}$ & Nicotiana tabacum \\
\hline $\mathrm{O} 2$ & Opaque 2 \\
\hline OHP1 & $\mathrm{O} 2$ heterodimerising protein 1 \\
\hline $\mathrm{P} 2 \mathrm{H}$ & Protoplast two- hybrid \\
\hline $\mathrm{P} 3 \mathrm{H}$ & Protoplast three- hybrid \\
\hline PBF & prolaminbox binding factor \\
\hline Pro & proline \\
\hline ProDH & proline dehydrogenation \\
\hline qPCR & qualitative polymerase chain reaction \\
\hline RNA & ribonuclein acid \\
\hline SIRT & sucrose induced repression of translation \\
\hline SNF1 & sucrose non- fermenting 1 \\
\hline SnRK1 & SNF1- related protein kinase 1 \\
\hline SSP & Seed storage protein \\
\hline $\mathrm{TF}$ & transcription factor \\
\hline uORFs & upstream open reading frames uORFs \\
\hline $\mathrm{Y} 2 \mathrm{H}$ & Yeast two- hybrid \\
\hline
\end{tabular}




\section{Danksagung}

Als Abschluss dieser Arbeit möchte ich mich bei allen, die mich bei meiner Doktorarbeit unterstützt haben, bedanken.

An allererster Stelle möchte ich mich ganz herzlich bei PD Dr. Wolfgang Dröge-Laser für das super Thema, für seine Unterstützung, für seine Betreuung und Motivation seit meiner Diplomarbeit bedanken.

Mein besonderer Dank gilt Prof. Dr. Christiane Gatz für Ihre Unterstützung und für die Möglichkeit diese Arbeit in der Abteilung anfertigen zu können, sowie für die Übernahme des Korreferats.

Ein riesig großes Dankeschön möchte ich Fridtjof Weltmeier für seine (Vor-) Arbeit und Katrin Dietrich für ihre Arbeit an unserem großen AtbZIP- Thema aussprechen.

Ein besonderer Dank geht an unsere AtbZIP Kollaborationspartner nach Madrid zu Rosario Alonso und Dr. Jesus Vicente-Carbajosa; nach Utrecht zu Dr. Micha Hanssen, Prof. Dr. Sjef Smeekens und Dr. Johannes Hanson, und nach Tübingen zu Prof. Dr. Klaus Harter für Ihre tolle Zusammenarbeit an unserem gemeinsamen Thema.

Für das tolle und vor allem lustige Laborklima möchte ich mich bei meinen anderen Laborkollegen (Stefan Böttner, Caroline Carsjens, Tim Iven, Christof Weiste, Nora Glaser, Laura Hartmann und Jennifer Krüger (TA)) ganz herzlich danken - wir sind schon eine coole Truppe $\left.;^{\circ}\right)$

Bedanken möchte ich mich auch bei meinen Laborkollegen aus der AG Gatz, für ihre Unterstützung bei Fragen oder Problemen.

Ein ganz besonders GROSSES Dankeschön geht an meinen Verlobten Arne, an meine und seine Familie für die großartige Unterstützung und Rückhalt nicht nur während dieser Arbeit, sondern auch während der schweren Stunden meiner Krankheit. 